\title{
Binary Mixtures and Fluids in the presence of Quenched Disorder
}

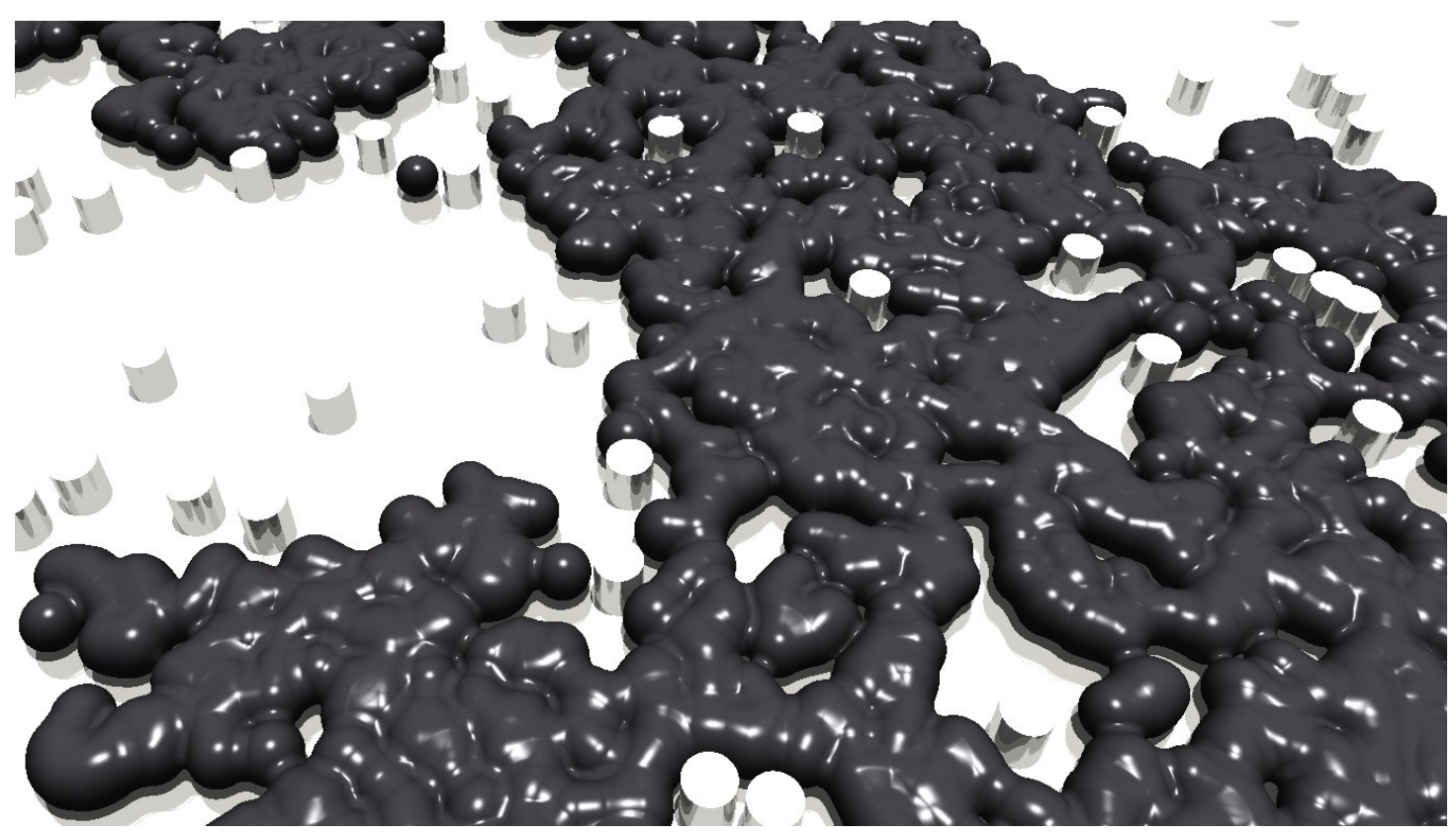

Dissertation

zur Erlangung des Doktorgrades der

Mathematisch-Naturwissenschaftlichen Fakultäten

der Georg-August-Universität Göttingen

vorgelegt von

Timo Daniel Fischer

Göttingen 2011 
D7

Referent: Marcus Müller

Koreferent: Dr. Richard L. C. Vink

Tag der mündlichen Prüfung: 18. Januar 2012 


\section{Acknowledgments}

The work presented in this thesis has been performed at the institute of theoretical physics at the University of Göttingen over the course of roughly three and a half years. I really enjoyed this time and cannot remember any negative experience with anyone. Therefore, I want to thank all the students, secretaries, technicians, and scientists of the institute of theoretical physics for a professional, friendly, and productive environment.

A few people directly related to this work require to be mentioned explicitly. Naturally, the first one of those is my thesis advisor Richard Vink whose Emmy-Noether grant not only paid for most of my living expenses, but who also has been a great tutor. His constant remainders to prefer a good result now over an excellent result never were a great help in getting things done. Similarly, I want to thank Annette Zippelius for funding in 2011. Finally, Till Kranz' careful proof-reading of this manuscript has been a great help in removing some embarassing typos. 



\section{Contents}

1 Introduction 1

2 Model and Simulation Basics 5

2.1 The Widom-Rowlinson Model . . . . . . . . . . . . . . . . . 5

2.2 Monte Carlo Method . . . . . . . . . . . . . . . . . . . 11

3 Data Analysis $\quad 19$

3.1 The Order Parameter Distribution . . . . . . . . . . . . . . . . . 19

3.2 Finite-size Scaling . . . . . . . . . . . . . . . . . 25

3.3 Constructing Loci $\ldots \ldots \ldots \ldots$. . . . . . . . . . . . . . . 31

4 Data Extrapolation $\quad 33$

4.1 The Histogram Method . . . . . . . . . . . . . . . . . . . . . 33

4.2 Uniform Sampling . . . . . . . . . . . . . . . . . . . . . 35

4.3 Temperature Reweighting . . . . . . . . . . . . . . . . . . 40

4.4 Summary . . . . . . . . . . . . . . . . . . . . . 46

5 Phase Coexistence and Sampling Barriers 49

5.1 Phase Coexistence . . . . . . . . . . . . . . . . . . . . . . . 49

5.2 Simulations on Hyperspheres . . . . . . . . . . . . . . . . . . . 53

5.3 Other Models . . . . . . . . . . . . . . . . . . . . 62

6 Random Neutral Obstacles $\quad 65$

6.1 Simulation . . . . . . . . . . . . . . . . 66

6.2 Disorder Average . . . . . . . . . . . . . . . . . . 66

6.3 Results. . . . . . . . . . . . . . . . . . . . 68

7 3D Mixtures with

$\begin{array}{lr}\text { Asymmetric Obstacles } & \mathbf{7 5}\end{array}$

7.1 Simulation . . . . . . . . . . . . . . . . . . . 75 


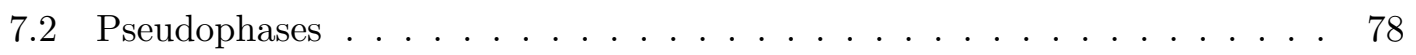

7.3 Extrapolating Pseudocritical Fugacities . . . . . . . . . . . . . . . 79

7.4 Modified Hyperscaling . . . . . . . . . . . . . . . . . . 81

7.5 Summary for 3D Mixtures . . . . . . . . . . . . . . 86

8 2D Fluids and Mixtures

$\begin{array}{ll}\text { with Asymmetric Obstacles } & 87\end{array}$

8.1 Choice of Quenched Disorder . . . . . . . . . . . . . . . 88

8.2 Elimination of the Liquid $\leftrightarrow$ Gas Transition . . . . . . . . . . . . . . . 88

8.3 Structural Properties: Domain Formation . . . . . . . . . . . . . . . . 94

8.4 Real-world Systems: Cell Membranes . . . . . . . . . . . . . . . . . 100

8.5 Summary: Elimination of a Phase Transition . . . . . . . . . . . . . . 110

$\begin{array}{lll}9 & \text { Summary } & 111\end{array}$

A Derivatives of the

Free Energy

113 


\section{Introduction}

The condensation and evaporation of a fluid, the transition between its liquid and its gas state, is a first order phase transition up to a maximum temperature, the critical temperature $T_{\mathrm{c}}$. Precisely at the critical temperature, at the critical point, the phase transition between liquid and gas is continuous. Above the critical temperature, the fluid is in the supercritical state, which is characterized by states of homogeneous composition and a density that continuously changes with the chemical potential. This behavior is in contrast to that below the critical temperature, where high density liquid and low density gas phases can coexist, and where the density as a function of the chemical potential is discontinuous at the phase transition between liquid and gas. From theoretical considerations, as well as experimental results, it is established that the critical point of the liquid $\leftrightarrow$ gas phase transition belongs to the universality class of the Ising model ${ }^{1 ; 2}$. This implies that close to the critical point observables scale with critical exponents that are characteristic of the Ising universality class and do not depend on the "system details" ${ }^{3}$. For example, measurements on sulfurhexaflouride and ${ }^{3} \mathrm{He}$ reported the critical exponents $\beta=0.327(6)$ and $\beta=3.271(6)$, respectively ${ }^{3 ; 4 ; 5}$. Similarly, binary mixtures of particles with short-ranged orientation-independent interactions also have a critical temperature: Above this temperature, homogeneous mixtures exist for all mole fractions. Below, some ratios of the mole fractions lead to a demixing into two coexisting phases that differ in composition ${ }^{1}$.

In the Ising model, related models can be constructed by adding quenched disorder in the form of random "impurities". Examples of such random impurities are the removal of randomly-selected Ising spins from the system and the addition of random static location dependent external magnetic fields, which result in the site-diluted Ising model ${ }^{6}$ and the the random-field Ising $\operatorname{model}^{7}$ (RFIM), respectively. Such Ising models with quenched disorder may have different critical behavior for the transition between paraand ferromagnetic phase, as in case of weak random fields in $d=3$ dimensions $^{8 ; 9}$. In some cases, such as random fields in $d=2$ dimensions ${ }^{10}$ or large amount of random site dilutions ${ }^{11}$, quenched disorder can even eliminate the the existence of a ferromagnetic phase altogether.

Since fluids and binary mixtures are in the universality class of the Ising model, similar results may hold true for such systems in a volume with random static inhomogeneities, such as the random wall structure formed by aerogels ${ }^{12}$. Indeed, it has been suggested 
by de Gennes that such confining random geometries should act on an immersed mixture in the same way that a random static and location dependent magnetic field acts on the Ising model ${ }^{13}$. This not only means that the behavior of the system is influenced by the presence of the inhomogeneities (as is seen in experiments ${ }^{14}$ ). More importantly, it also suggests that the properties of a macroscopic demixing (or a liquid $\leftrightarrow$ gas transition) may be "universal" in the sense that they are determined by only a few basic characteristics of the inhomogeneities. In analogy to the Ising model, such characteristics may be the spatial symmetry of the inhomogeneities, the range of their interaction with the mobile components, and whether a preferred affinity to one of the potentially coexisting phases exists.

Since for a fluid an exactly equal affinity of the inhomogeneities to the liquid and the gas phase is unlikely, the expected universality class would be that of the random-field Ising model. Yet, in experiments on ${ }^{4} \mathrm{He}$ and $\mathrm{N}_{2}$ in silica aerogels, de Gennes' hypothesis of RFIM universality could not be confirmed. Critical exponents compatible with those of the unperturbed Ising model were reported, instead ${ }^{15 ; 16}$. As a possible explanation of this discrepancy, it has been suggested that an aerogel may be better described as a system with random impurities that have long ranged correlations ${ }^{15 ; 16}$, in which case RFIM universality would not apply ${ }^{17}$. However, another possibility could be a crossover effect, as in RFIM systems with weak random fields, the true critical behavior is only seen very close to the critical temperature, while further away the normal Ising universality is seen $^{8}$.

In this thesis, results from large scale computational studies are presented that provide further evidence for the applicability of de Gennes' hypothesis. To this end, results from Monte Carlo simulations of the Widom-Rowlinson model ${ }^{18}$ in the presence of different types of static inhomogeneities ("obstacles") are presented and extrapolated to the thermodynamic limit via finite-size scaling methods ${ }^{19 ; 20}$. For random inhomogeneities this requires investigating many different possible arrangements of the inhomogeneities, which increases the computational effort and the complexity of the data analysis. To handle thousands of inhomogeneity configurations, automatized data analyses have been developed, which also forms a main ingredient of this work.

The outline of this thesis is as follows: In chapters 2 the Widom-Rowlinson model is being introduced, which is a model that can be interpreted as a fluid model and a binary mixture model at the same time, and therefore allows to draw conclusions about both types of systems. The Monte Carlo method is briefly introduced, and a possible Monte Carlo algorithm for the simulation of the Widom-Rowlinson model is presented. In chapter 3 , a finite-size scaling analysis of fluid simulation data is introduced, which foots on the works of Fisher ${ }^{21 ; 22}$ and Binder ${ }^{23}$. Chapter 4 introduces advanced Monte Carlo methods that are required to obtain useful data with a sensible amount of computing effort ${ }^{\mathrm{a}}$. A

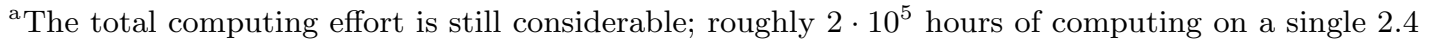
$\mathrm{GHz}$ CPU for the results presented here. The effort is merely reduced from "impossible" to "high".
} 
side project that has resulted from the intensive preoccupation with the development of efficient simulations and data analyses is presented in chapter 5 . There, it is demonstrated that simulation studies of first order phase transitions in fluids can benefit from the choice of an unconventional choice of the system geometry, namely the surface of a hypersphere.

The main results are presented in chapters 6,7 , and 8. In chapter 6 the simplest case of random static inhomogeneities, those which equally affect both possibly-coexisting phases, is investigated. On the example of a two dimensional system it is shown that such (weak) inhomogeneities affect the model such that its universal behavior is consistent with the universality of the site-diluted Ising model. This implies that there still exists a first-order phase transition that ends in a critical point, but with the critical temperature reduced compared to that of the model without inhomogeneities (the "pure model").

The case of random inhomogeneities that interact differently with the two components of a three dimensional binary mixture is discussed in chapter 7 . Standard methods and a new finite-size scaling technique proposed by Binder ${ }^{24}$ are used for this case. In consistence with previous simulations of similar scope ${ }^{25 ; 26}$ (using finite-size scaling and considering many different inhomogeneity configurations) it is found that the demixing transition still exists, and that the critical temperature is reduced compared to that of the pure model. By comparison with simulations of the random-field Ising model it is argued that the universality of the critical point is indeed that of the RFIM, which implies an unconventional critical point that violates the hyperscaling relation. Signatures of the existence of a third phase that have been reported from other computational studies ${ }^{27}$ are explained as a finite-size artifact and the result of not taking into account a sufficient amount of inhomogeneity configurations.

The corresponding two dimensional system is discussed in chapter 8. For this case, the absence of a ferromagnetic phase in the 2D RFIM ${ }^{8}$ implies the absence of a demixing phase transition, whereas simulations of grid-based models have reported the existence of a demixing transition ${ }^{28}$. By investigation of systems of different size it is argued that the indications of a first-order phase transition seen in the raw simulation data are a finite size effect, and that in the thermodynamic limit the demixing transition (or liquid $\leftrightarrow$ gas transition) is eliminated by the inhomogeneities. Instead, a continuous freezing into structures dictated by the inhomogeneities is found and argued to be the effect that eliminates and replaces the phase transition.

Finally, an important potential application of the results found for the two dimensional mixtures in the presence of random static inhomogeneities is discussed in the second part of chapter 8 . This application concerns the lateral structure of lipid bilayers, such as membranes of eukaryotic cells. While in vesicles formed from the phospholipids of eukaryotic cells a demixing transition compatible with the Ising universality class is being observed ${ }^{29}$, no such transition has been reported in membranes of live cells. Footing on a hypothesis that explains these effects as random site dilutions caused by cytoskeleton bound proteins in the membrane ${ }^{30}$, in this thesis it is instead argued that a 
random-field effect is more likely. Such a random-field effect could explain the existence of lipid rafts ${ }^{31 ; 32 ; 33}$ as an equilibrium effect without requiring proximity to a critical point. Molecular dynamics simulations of a model membrane with two different species of phospholipids at near atomic resolutions are presented which are shown to be consistent with the results obtained from the Monte Carlo simulation of the Widom-Rowlinson mixture. 


\section{Model and Simulation Basics}

As the model system to investigate in the presence of a background with random static inhomogeneities the Widom-Rowlinson model ${ }^{18}$ (WR model) has been chosen. The WR model has two key features that make it an interesting choice to consider: From the point of view of a programmer the model components and interactions are simple to implement and can be simulated efficiently with simple Monte-Carlo steps (the insertion and removal of single particles described in section 2.2.2.1). For a physicist it is interesting that the WR model is a model for a binary mixture and a single-species fluid at the same time in the sense that an exact mathematical transcription rule (a mapping) between both interpretations exists (section 2.1.2). In many cases, this will allow drawing conclusions for the fate of the liquid $\leftrightarrow$ gas transition of a quenched fluid and the demixing transition of a quenched binary mixture from the same data.

Random static inhomogeneities in the system are modeled in the form of randomly placed static particles called obstacles. But before the case of quenched disorder is being discussed, the Monte Carlo simulation method, a suitable finite-size scaling analysis (chapter 3), and advanced Monte Carlo techniques (chapter 4 and 5) need to be introduced. These methods are being discussed on the example of the original WR model without obstacles (the pure model). The respective results for the pure model are presented alongside. Since the critical exponents of the Ising universality are known to a good degree of accuracy, and since independent computer simulations of the pure WR model exist, this also provides a non-trivial test of the applicability and the proper implementation of these concepts.

\subsection{The Widom-Rowlinson Model}

\subsubsection{The Widom-Rowlinson as a Binary Mixture}

The WR model is best introduced in its interpretation as a binary mixture, the WidomRowlinson mixture. The mixture consists of two particle species, A and B, which are both spheres with diameter equal to one unit of length. The only interaction is a hard-core interaction between particles of unlike species, i.e. overlaps of A particles with B particles are forbidden while particles of the same species may freely overlap. For a $d$-dimensional system of volume $V$ with $N_{\mathrm{A}}$ and $N_{\mathrm{B}}$ particles of species A and B the microcanonical 
partition function can formally be expressed as

$$
\Omega\left(V, N_{\mathrm{A}}, N_{\mathrm{B}}\right)=\frac{1}{N_{\mathrm{A}} ! N_{\mathrm{B}} !} \int_{V} \mathrm{~d}^{d N_{\mathrm{A}}} x \mathrm{~d}^{d N_{\mathrm{B}}} y \underbrace{\prod_{i=1}^{N_{\mathrm{A}}} \prod_{j=1}^{N_{\mathrm{B}}} \theta\left(\left\|\mathbf{x}_{i}-\mathbf{y}_{j}\right\|-1\right)}_{=: X(x, y)},
$$

where the term $\left\|\mathbf{x}_{i}-\mathbf{y}_{j}\right\|$ is to be understood as the distance between the $i$-th $\mathrm{A}$ and the $j$-th B particle, and $\theta$ is the usual Heaviside step function. The exclusion term $X$ therefore discards all microstates with an overlap of A and B particles from the sum.

The system defined by the partition function (2.1) does not contain any kinetics for the particles. The extra terms for the momenta would be trivial and can be integrated out resulting in just an extra analytic temperature-dependent term to carry around. For simplicity and focus on the interesting part of the mixture, the spatial configurations, the kinetics have been omitted here ${ }^{\mathrm{a}}$. The grand canonical partition function with the fugacities $z_{\mathrm{A}}$ and $z_{\mathrm{B}}$ as parameters is

$$
\mathcal{Z}\left(V, z_{\mathrm{A}}, z_{\mathrm{B}}\right)=\sum_{N_{\mathrm{A}}, N_{\mathrm{B}}} z_{\mathrm{A}}^{N_{\mathrm{A}}} z_{\mathrm{B}}^{N_{\mathrm{B}}} \Omega\left(V, N_{\mathrm{A}}, N_{\mathrm{B}}\right)
$$

Since there is no explicit temperature in the mixture picture, the logarithm of the fugacities will be called the chemical potentials, i.e.

$$
\mu_{\mathrm{A}}:=\log z_{\mathrm{A}}, \text { and } \mu_{\mathrm{B}}:=\log z_{\mathrm{B}} .
$$

\subsubsection{The Widom-Rowlinson Model as a Fluid}

The Widom-Rowlinson model can also be understood as a single species fluid with a hidden symmetry, the Widom-Rowlinson fluid. To map the binary mixture on a fluid, first re-interpret the mixture as a mixture of A particles with diameter two, and B particles with diameter zero. This blurs the symmetry of the model, but leaves the partition function unchanged. By integrating out the B particles' degrees of freedom in the grand canonical ensemble, the partition function of the WR mixture,

$$
\mathcal{Z}\left(V, z_{\mathrm{A}}, z_{\mathrm{B}}\right)=\underbrace{\sum_{N_{\mathrm{A}}} \frac{z_{\mathrm{A}}^{N_{\mathrm{A}}}}{N_{\mathrm{A}} !} \int_{V} \mathrm{~d}^{d N_{\mathrm{A}}} x}_{\text {A particles }} \underbrace{\sum_{N_{\mathrm{B}}} \frac{z_{\mathrm{B}}^{N_{\mathrm{B}}}}{N_{\mathrm{B}} !} \int_{V} \mathrm{~d}^{d N_{\mathrm{B}}} y}_{\text {B particles }} X(x, y)
$$

maps onto the partition function of a single-species fluid. To see this, consider a fixed number and configuration for the A particles. For this configuration, the term $X(x, y)$ creates a volume $V_{X}(x)$, in which no B particle can be located. Except for this volume

\footnotetext{
${ }^{a}$ For a short description of model and mapping that involves the kinetics see e.g. Miguel et al. ${ }^{34}$.
} 
exclusion, there is no restriction on the B particles. They are an ideal gas in the remaining volume $V-V_{X}(x)$, and their degrees of freedom can be integrated out:

$$
\begin{aligned}
\mathcal{Z}\left(V, z_{\mathrm{A}}, z_{\mathrm{B}}\right) & =\sum_{N_{\mathrm{A}}} \frac{z_{\mathrm{A}}^{N_{\mathrm{A}}}}{N_{\mathrm{A}} !} \int_{V} \mathrm{~d}^{d N_{\mathrm{A}}} x \sum_{N_{\mathrm{B}}} \frac{z_{\mathrm{B}}^{N_{\mathrm{B}}}}{N_{\mathrm{B}} !} \int_{V-V_{X}(x)} \mathrm{d}^{d N_{\mathrm{B}}} y 1 \\
& =\sum_{N_{\mathrm{A}}} \frac{z_{\mathrm{A}}^{N_{\mathrm{A}}}}{N_{\mathrm{A}} !} \int_{V} \mathrm{~d}^{d N_{\mathrm{A}}} x \underbrace{\sum_{N_{\mathrm{B}}} \frac{z_{\mathrm{B}}^{N_{\mathrm{B}}}}{N_{\mathrm{B}} !}\left(V-V_{X}(x)\right)^{N_{\mathrm{B}}}}_{=\exp \left[z_{\mathrm{B}}\left(V-V_{X}(x)\right)\right]} \\
& =\sum_{N_{\mathrm{A}}} \frac{z_{\mathrm{A}}^{N_{\mathrm{A}}}}{N_{\mathrm{A}} !} \int_{V} \mathrm{~d}^{d N_{\mathrm{A}}} x e^{-z_{\mathrm{B}}\left(V_{X}(x)-V\right)} .
\end{aligned}
$$

With all degrees of freedom of the B particles eliminated, the partition function can be interpreted as the partition function for a model containing only one species of particles (A particles) at an inverse temperature of $\beta=z_{\mathrm{B}}$ that has a binding energy term $V_{X}(x)-V$. While this binding energy is not expressed as the sum of one- and two-particle potentials, the $V_{X}-V$ term is a short ranged attractive potential. It is attractive in the sense that the closer overlapping particles move together, the less area they cover, and the lower the potential energy of the state becomes. And it is short ranged in the sense that non-overlapping clusters of A particles (or just single particles) can be moved around freely without changing the potential energy. On a more technical note, the potential is bound from above (since $\left.V_{X} \leq V\right)$ and more importantly from below $\left(V_{X} \geq 0\right)$, which prevents the state from simply collapsing towards the state with infinite negative energy. This latter property is required for the system to show true thermodynamics ${ }^{18}$, since it allows entropy to compete with energy.

Because of these properties of the potential, the partition function in equation 2.6 is considered the partition function for a one-species fluid with attractive interactions. The WR model therefore describes a binary mixture and a single-particle fluid at the same time, where mapping from the mixture to the fluid is essentially done by simply ignoring the presence of the B particles. Since the WR fluid contains an energy term and an inverse temperature the grand canonical partition function may be rewritten as

$$
\begin{aligned}
\mathcal{Z}\left(V, z_{\mathrm{A}}, z_{\mathrm{B}}\right) & =\sum_{N_{\mathrm{A}}, N_{\mathrm{B}}} z_{\mathrm{A}}^{N_{\mathrm{A}}} z_{\mathrm{B}}^{N_{\mathrm{B}}} \Omega\left(V, N_{\mathrm{A}}, N_{\mathrm{B}}\right) \\
& =\sum_{N_{\mathrm{A}}} z_{\mathrm{A}}^{N_{\mathrm{A}}} \underbrace{\sum_{N_{\mathrm{B}}} z_{\mathrm{B}}^{N_{\mathrm{B}}} \Omega\left(V, N_{\mathrm{A}}, N_{\mathrm{B}}\right)}_{=: Z\left(V, N_{\mathrm{A}}, z_{\mathrm{B}}\right)} \\
& =\sum_{N_{\mathrm{A}}} z_{\mathrm{A}}^{N_{\mathrm{A}}} Z\left(V, N_{\mathrm{A}}, z_{\mathrm{B}}\right),
\end{aligned}
$$

where $Z$ is the canonical partition function for the fluid with $N_{\mathrm{A}}$ particles at an inverse temperature $z_{\mathrm{B}}$. 


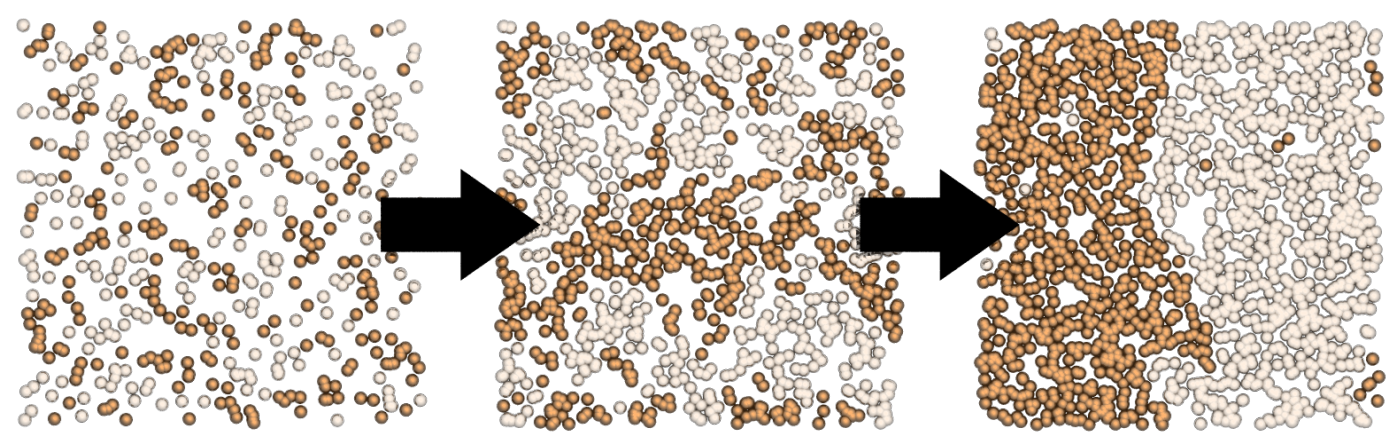

Figure 2.1: Effects of increasing the density in the two-dimensional Widom-Rowlinson model of a square with periodic boundary conditions. At low densities (left image for $\rho_{\mathrm{A}}=\rho_{\mathrm{B}}=0.3$ ) both species are mixed. Upon increasing the density (center image, $\rho_{\mathrm{A}}=\rho_{\mathrm{B}}=0.6$ ) clusters of increasing size form, which are caused by the vicinity to critical point and are called near-critical fluctuations. Above the critical density, the particles have de-mixed into two macroscopic clusters with an interface parallel to the system boundaries (right image, $\rho_{\mathrm{A}}=\rho_{\mathrm{B}}=0.9$ ).

\subsubsection{The Phase Diagram}

In figure 2.1 typical states for the two dimensional WR mixture at different densities are seen (the three dimensional case is equivalent). For very low densities, the particles mix. If the density is increased, clusters of particles develop that grow in size as the density is increased further. At some point, the size of these clusters diverges, and a macroscopic demixing occurs, in which the system volume is covered by two spatially separated phases ${ }^{\mathrm{b}}$. One of the phases is rich in A particles and lean in B particles (the A phase), the other phase has reversed composition (the B phase). The corresponding behavior in the grand canonical ensemble is shown in figure 2.2. At fugacities far below a critical fugacity, the system is in a mixed state. Upon increasing the fugacities towards the critical one, growing clusters appear whose size diverges at the critical point. However, contrary to the microcanonical ensemble no coexistence of two phases is seen for fugacities above the critical fugacity. Instead, the system uniformly exhibits either the A or the B phase, but not both at the same time (not even if $z_{\mathrm{A}}=z_{\mathrm{B}}$, since coexistence is suppressed by an interface contribution to the free energy - see chapter 5 ).

The schematic phase diagrams for the Widom-Rowlinson model in the microcanonical ensemble, in the canonical ensemble (of the fluid interpretation), and the grand canonical ensemble are shown in figure 2.3 (a), (b), and (c), respectively. In the microcanonical ensemble, mixed and macroscopically demixed states are separated by a demixing phase transition in the plane of the densities $\rho_{\mathrm{A}}=N_{\mathrm{A}} / V$ and $\rho_{\mathrm{B}}=N_{\mathrm{B}} / V$. The phase diagram of the canonical ensemble is that of a fluid that can undergo a liquid $\leftrightarrow$ gas phase transition

\footnotetext{
${ }^{\mathrm{b}}$ Of course, the largest attainable length scale in a computer simulation is that of the small system that is simulated. In simulations, a macroscopic demixing can only be indicated, but not actually occur.
} 


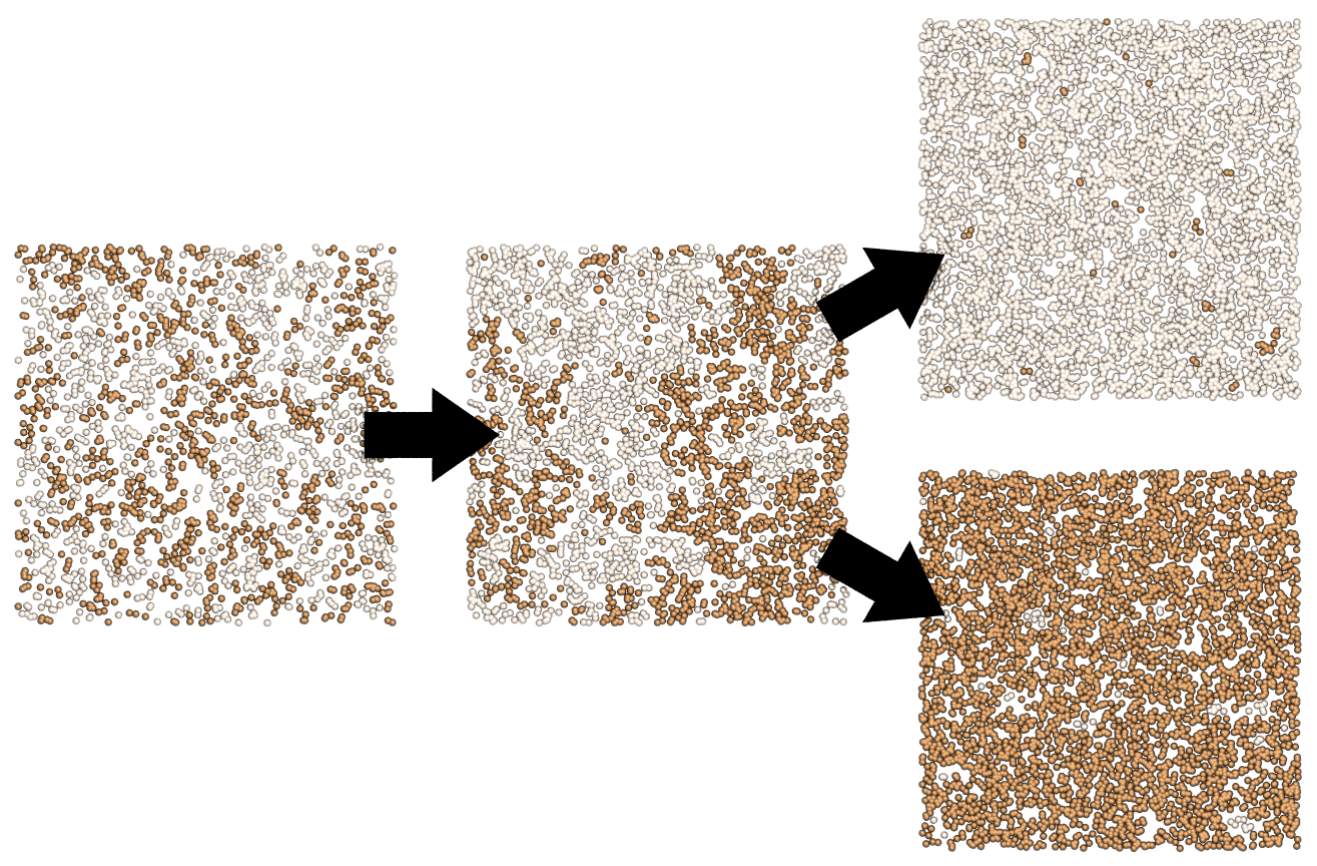

Figure 2.2: Effect of increasing the fugacity of the WR mixture in the grand canonical ensemble. For low fugacities (left image at $z_{\mathrm{A}}=z_{\mathrm{B}}=1$ ) a mixed state is seen. When the fugacity is increased, domains of $\mathrm{A}$ and $\mathrm{B}$ particles develop that grow as the critical fugacity is approached (middle state, $\left.z_{\mathrm{A}}=z_{\mathrm{B}}=1.5\right)$. Above the critical fugacity, the system is either in a state containing almost only A particles (the A phase) or in a state containing almost only B particles (the B phase), as shown in the two rightmost snapshots at $z_{\mathrm{A}}=z_{\mathrm{B}}=2$.
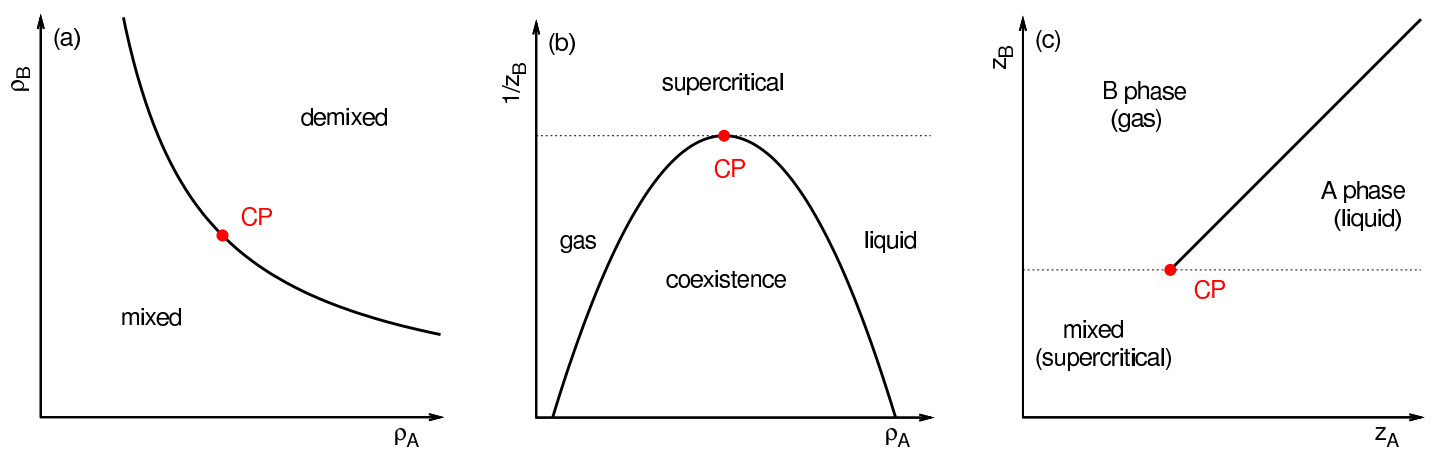

Figure 2.3: Schematic phase diagrams for the WR model in (a) the microcanonical ensemble, (b) the fluid canonical ensemble, (c) and the grand canonical ensemble. Physical phase boundaries are drawn in a solid line, the unphysical boundary to the supercritical phase is drawn with a dashed line, the critical point is indicated with a red dot. The labels in figure (a) and (b) refer to the mixture and the fluid picture of the WR model, respectively. In figure (c), the names of the phases are given in both pictures. The border of the supercritical phase is drawn as a dashed line to indicate that there is no phase transition associated with it. 


\begin{tabular}{|c|c|c|c|c|c|}
\hline \multirow{2}{*}{\multicolumn{2}{|c|}{ Theory }} & \multicolumn{2}{|c|}{$2 \mathrm{D}$} & \multicolumn{2}{|c|}{$3 \mathrm{D}$} \\
\hline & & $\rho_{\mathrm{cr}}$ & $z_{\mathrm{B}, \mathrm{cr}}$ & $\rho_{\mathrm{cr}}$ & $z_{\mathrm{B}, \mathrm{cr}}$ \\
\hline 1970 & mean field th. ${ }^{18}$ & 0.637 & - & 0.4775 & - \\
\hline 1976 & scaled particle th. ${ }^{35}$ & - & - & 0.4347 & - \\
\hline \multirow[t]{2}{*}{2000} & density functional th. ${ }^{36}$ & - & - & 0.556 & - \\
\hline & MC Simulations & & & & \\
\hline 1996 & Semigrandcanonical $^{37}$ & - & - & $0.762(16)$ & - \\
\hline 1997 & Cluster flipping ${ }^{38}$ & $1.566(3)$ & $1.726(2)$ & $0.748(2)$ & $0.9403(1)$ \\
\hline 2011 & (this work) & $1.562(5)$ & $1.718(2)$ & $0.752(4)$ & $0.9373(6)$ \\
\hline
\end{tabular}

Table 2.1: Notable previous estimates for the critical density and the critical fugacity of the WR model. While the Monte Carlo results essentially agree on all properties, various theoretical approximations all disagree and also underestimate the critical density.

(note that $1 / z_{\mathrm{B}}$ is the temperature in this interpretation). Above a critical temperature (for $1 / z_{\mathrm{B}}>1 / z_{\mathrm{B}, \mathrm{cr}}$, where $z_{\mathrm{B}, \mathrm{cr}}$ is the inverse critical temperature), the fluid can exhibit any uniform density. This characterizes the supercritical phase. Below the critical temperature, two distinct density regimes exist: the liquid phase and the gas phase. Intermediate total densities are possible, but do not result in a uniform state but in the coexistence of appropriate amounts of the liquid and the gas phase. In the grand canonical ensemble the system exhibits a mixture of $\mathrm{A}$ and $\mathrm{B}$ particles below the critical fugacity, i.e. for $z_{\mathrm{B}}<z_{\mathrm{B}, \mathrm{cr}}$. Above the critical fugacity, the system is in either the $\mathrm{A}$ or the $\mathrm{B}$ phase, but never in a state of coexistence between $\mathrm{A}$ and $\mathrm{B}$ phase. Instead, at the phase transition line (that because of the WR mixture's symmetry must lie at $z_{\mathrm{A}}=z_{\mathrm{B}}$ ) a phase transition between $\mathrm{A}$ and $\mathrm{B}$ phase occurs. The transition is first order and ends in an Ising critical point.

The existence of a phase transition ending in a critical point was already suggested when the model was first presented ${ }^{18}$ and proven shortly thereafter ${ }^{39}$. But determining the location of the critical point has, at least in two and three spatial dimension, been a problem (see table 2.1). Originally, Widom and Rowlinson used a mean-field ansatz ${ }^{18}$, which should become accurate in the limit of infinite dimensions. Attempts to improve this result by more sophisticated theoretical methods resulted in a scatter of around $20 \%$ of the original estimate, but with no systematic trend. The first computer experiment performed on the WR model ${ }^{37}$, however, reported a critical density that is incompatible with any of these theoretical results, and has since been confirmed in other computer simulations $34 ; 38 ; 40 ; 41$ - and also agree with my results. As expected for a fluid with short ranged interactions, the model has been found to lie in the universality class of the Ising model $^{38 ; 42}$. 


\subsubsection{Related Models}

From a more abstract point of view, the Widom Rowlinson mixture is just a point in the parameter space of the more general class of non-additive hard sphere binary mixtures, which have a demixing for sufficiently large positive non-additivity ${ }^{35}$ (but in some cases this critical density can lie within the solid regime ${ }^{43}$ ). The Widom Rowlinson mixture is the special case of both particles having radius zero, which for example allows for the mapping on the fluid model.

Another relation to other models can be constructed by axis-parallel cubes instead of spheres. On the level of the partition functions the only difference is that the $L_{2}$ norm $\|\mathbf{x}\|=\left(\left|x_{1}\right|^{2}+\cdots+\left|x_{d}\right|^{2}\right)^{1 / 2}$ in the exclusion term $X$ in equation (2.1) is replaced by the $L_{\infty}$ norm $\|\mathbf{x}\|=\max \left(\left|x_{1}\right|, \ldots,\left|x_{d}\right|\right)$. Other than the microscopic geometry of the particles and the volume occupied by them, the main properties of this model remain that of the Widom Rowlinson model - including the mapping on a single-species fluid with attractive interactions.

In two dimensions this model of axis-parallel squares is also equivalent to a system of unit length horizontal and vertical rods of zero width that may not intersect, the two-dimensional Zwanzig model ${ }^{44}$. Recently, simulations of lattice models with horizontal and vertical rods have been performed, in which a transition from a mixed state to a state with only one of the rod species has been seen ${ }^{45 ; 46 ; 47}$ and identified as an Ising type phase transition ${ }^{48}$. Because of the similarity of these lattice models to the $2 \mathrm{D}$ Zwanzig model (which can in some sense be considered the off-lattice version of such models) it has been argued by Richard Vink and $\mathrm{e}^{49}$ that the phase transition that is seen can also be understood as a transition from a supercritical state to a liquid or a gas phase rather than an isotropic $\leftrightarrow$ nematic type transition as it has originally been called ${ }^{45 ; 46 ; 47 ; 48}$.

\subsection{The Monte Carlo Method}

To evaluate ensemble averages for the Widom-Rowlinson model in the respective ensembles, Monte Carlo methods are used. Essentially, a Monte Carlo method is the approximation of an ensemble (a phase space, an integration domain, a thermodynamic ensemble, ...) by a randomly generated finite sequence of elements that are in some sense "representative" of the full ensemble. In some cases, the generation of such a representative sample can already be the sought-for result: In collider physics experiments computer programs called event generators ${ }^{50 ; 51}$ are employed to randomly generate a representative set of collision results that are used to optimize data analysis algorithms during the development stage of the experiment, and to compare predictions of a particle physics model with actual experimental results. In other cases, the reason for using the Monte Carlo method is that operations that cannot be performed on the full ensemble may be performed on the generated sequence instead, i.e. to approximate an operation's result by approximating 
the ensemble. An example of this kind of application is the numerical integration of a function over some domain (Monte Carlo integration ${ }^{52}$ ). The simplest way to perform this is to pick elements from the integration domain randomly with an equal probability $p(\gamma)=$ const. In this case, the integral is then approximated by evaluating the function on the points $\gamma_{i}$, taking the average of these values, and multiplying with the volume of the integration domain.

In this thesis, the reason for generating a sequence $\gamma_{1}, \gamma_{2}, \ldots, \gamma_{N}$ of states from the ensemble is the latter one: to calculate ensemble averages. In particular, ensemble averages of the grand canonical ensemble shall be evaluated. In statistical physics, one does not generate the sequence from a flat probability distribution, but instead takes the Boltzmann weight into account for the generation of the sequence. If one thinks of the Boltzmann weight as the relative probability for the state $\gamma$ to actually appear during a measurement, then a "representative" sequence is generated when the states are sampled with a relative probability that equals their Boltzmann weight ${ }^{53}$. In case of the WR mixture in the grand canonical ensemble, this means sampling from a probability distribution

$$
p(\gamma)=\frac{\text { const }}{N_{\mathrm{A}}(\gamma) ! N_{\mathrm{B}}(\gamma) !} z_{\mathrm{A}}^{N_{\mathrm{A}}(\gamma)} z_{\mathrm{B}}^{N_{\mathrm{B}}(\gamma)} \cdot X(\gamma),
$$

where $X(\gamma)$ is zero for all states $\gamma$ with an overlap between A and B particles, and one otherwise (i.e. represents the exclusion term $X(x, y)$ in equation 2.1). In such a biased sampling, which is called importance sampling, the Boltzmann weight is then not taken into account a second time for the calculation of ensemble averages. Instead, the approximates for ensemble averages $\langle f\rangle$ of a function $f(\gamma)$ take the simple form

$$
\langle f\rangle=\frac{1}{N} \sum_{i=1}^{N} f\left(\gamma_{i}\right) .
$$

\section{Markov Processes}

While ideally the $\gamma_{i}$ are mutually uncorrelated, an independent construction of states "from scratch" is usually very inefficient. For the WR mixture, an uncorrelated sequence of states can be generated by choosing random particle numbers $N_{\mathrm{A}}$ and $N_{\mathrm{B}}$ from suitable Poissonian probability distributions and inserting the respective amount of particles at random independent locations. By discarding the so-constructed states that contain an overlap between an A and a B particle (i.e. with $X(\gamma)=0$ ), a sequence of states according to the probability distribution (2.9) is generated. But as shown in figure 2.4(a), for system sizes considered here $(V>100), X(\gamma)=0$ for almost all constructed states and this method will essentially never result in the generation new entry in the sequence.

The solution to this problem is to give up the mutual independence of the sampled states. Instead of generating each state from scratch a Monte Carlo step can take the 

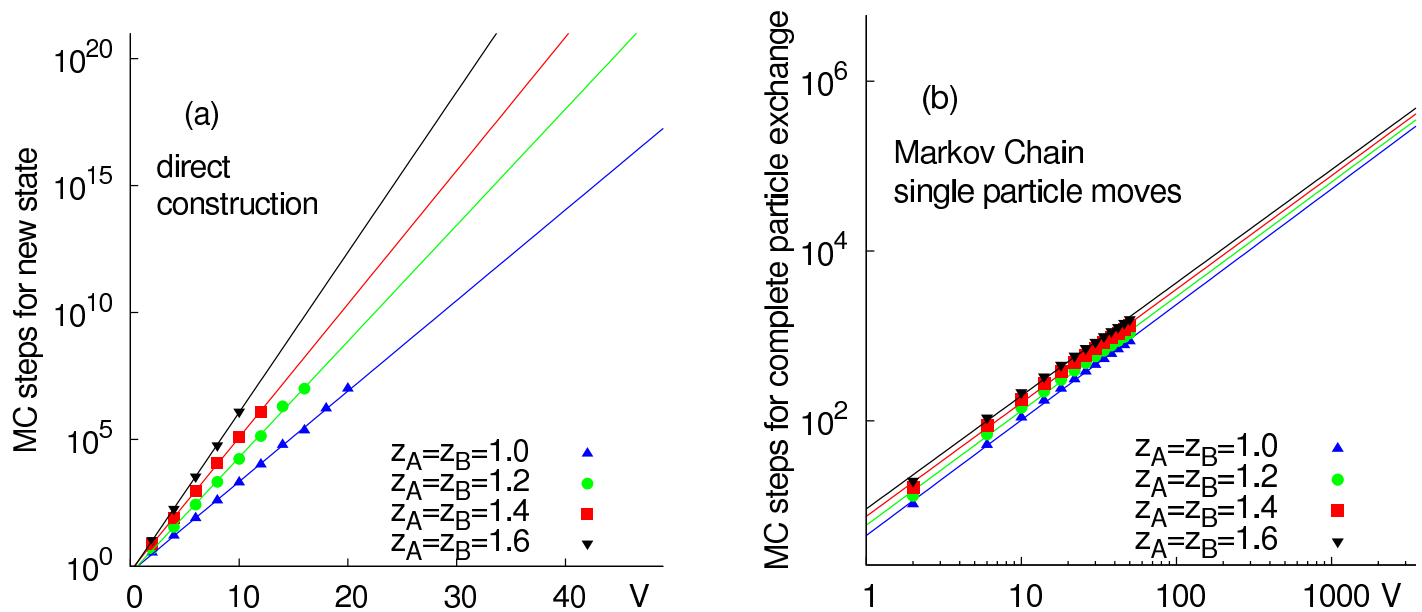

Figure 2.4: Efficiency for different methods to construct a Monte Carlo sequence for the two dimensional WR mixture. Figure (a) shows the average number of MC steps that a direct construction of states requires to construct a "legal" state without overlap between A and B particles (the construction algorithm used is explained in the text). Lines are fits on the data points. For relevant system sizes $V>100$, a computer simulation would take forever to construct even a single state this way. Figure (b) shows the average number of attempted MC steps that a Markov Chain simulation with the MC steps introduced in section 2.2.2.1 needs until all particles of the starting state have been replaced. Note the different scales on the two plots.

previously-constructed state $\gamma_{i-1}$ and randomly modify it. Thereby a new and in some sense similar state $\gamma_{i}$ is generated, that is correlated with $\gamma_{i-1}$ but also more likely to be a valid state (meaning that $X\left(\gamma_{i}\right)=1$ ). If the probability that a Monte Carlo step creates a target state $\nu$ only depends on the previously-generated state $\mu$ then this sampling formally is a Markov Process ${ }^{54}$ with a transition matrix $T_{\nu \mu}$, whose entries are the probabilities that $\gamma_{i+1}=\nu$ if $\gamma_{i}=\mu$ (note that $T_{\mu \mu} \neq 0$ is allowed). As long as the transition matrix satisfies two conditions explained in the following, ergodicity and detailed balance, then in the limit of a large number of sampled states the process samples states with a probability according to the Boltzmann distribution ${ }^{53}$.

\section{Ergodicity and Detailed Balance}

A sampling process is ergodic in the strict mathematical sense exactly if for each pair of states with a non-zero Boltzmann weight there is a non-zero probability to reach the respectively other state after a finite number of steps. Consequently, a process is not ergodic if there is a state of non-zero probability that cannot be reached from every other state of non-zero probability with a finite number of steps. Ergodicity is a global property of a Markov Process that ensures that states with a non-zero probability $p(\gamma)$ can possibly appear in the sequence. Detailed balance is a constraint on the entries of the transition 
matrix:

$$
T_{\nu \mu} p(\mu)=T_{\mu \nu} p(\nu)
$$

This is a local condition on the Markov process that, in combination with ergodicity, ensures that in the limit of a long sequence the states are generated according to the Boltzmann distribution.

Note that $(2.11)$ allows entries $T_{\mu \mu} \neq 0$, i.e. a finite probability that the new state is the same as the old state - being stuck in the state forever is ruled out by ergodicity. Also, entries $T_{\mu \nu}=0$ are allowed, even if $p(\mu) \neq 0$ and $p(\nu) \neq 0$. Ergodicity only demands that the Markov Process can diffuse from state $\mu$ to state $\nu$ and back, but it is not required that the transition happens in a single Monte Carlo step. Finally note that equation 2.11 implies that if a Monte Carlo step can lead from state $\mu$ to state $\nu$ (and both states have a non-zero sampling probability), then there must be a non-zero probability to go back from $\nu$ to $\mu$ : Monte Carlo steps obeying detailed balance must necessarily be able to "undo" themselves.

\subsubsection{Ergodicity Breaking}

The principles of ergodicity and detailed balance are paramount to the construction and understanding of Monte Carlo simulations in statistical physics, since they provide the mathematical backup on which the trust in the method foots. Yet, real simulations routinely violate either or both of these principles - in a controlled way. For example, ergodicity in the strict sense introduced above is neither required nor sufficient for a Monte Carlo simulation. For the WR mixture, it is (strictly speaking) not possible to represent all states of the partition function: A floating point variable cannot exactly represent a real-valued coordinate but merely stands for roughly $10^{16}$ discrete numbers that approximate the real values over some interval, and the necessarily finite amount of computer memory puts an upper limit to the number of particles that can be represented. Of course, one can expect that a grid with $10^{16}$ discrete values for each coordinate is a reasonable approximation for a continuum model and that particle numbers much larger than $z_{\mathrm{A}} V+z_{\mathrm{B}} V$ (the average number of particles in the model in case of the absence of the interaction) are irrelevant, anyways. A simulation may break ergodicity as long as the simulator is aware of these violations and can justify them as irrelevant to the result (or correct for them).

Similarly, ergodicity in the mathematical sense is not sufficient for a simulations, since ergodicity can be violated effectively. Effective ergodicity breaking can happen when two important regions of the phase space (important in the sense that both contribute significantly to the partition function) are connected by paths whose integrated probabilities are greater than zero but small. Since a simulation can only perform a finite number of Monte Carlo steps, this probability may be so small that the simulation does not transit between the two regions and only samples states from one of them. This typically 
results in a non-representative sequence. An example of such a potential bottleneck is the simulation of a system that undergoes a first order phase transition. At phase equilibrium, both phases are equally likely to be present in the system and hence need to be sampled. However, if the Monte Carlo steps that have been chosen can only move from the one phase to the other by sampling intermediate states in which the two phases coexist (that are very unlikely to appear in a simulation as explained in chapter 5), then the transition between the two phases is effectively eliminated ${ }^{c}$. Because of the uniform sampling methods that are introduced in chapter 4, this does not directly become a problem in the simulations presented here, but a remnant impact on ergodicity remains in form of "shape transitions" (see chapter 5).

\subsubsection{Generating Monte Carlo Steps}

The construction of an ergodic computer algorithm that satisfies the detailed balance condition (2.11) may seem like a daunting task. In practice, there is a trick that can be employed that greatly simplifies this task and allows for a large amount of flexibility: One chooses any algorithm that performs a random modification to the current state $\gamma_{i}$ such that the resulting Markov Chain is ergodic, but only accepts a proposed modification with a suitably chosen probability $P_{\text {acc }}(\mu \rightarrow \nu)$ and otherwise simply keeps the current state (i.e. $\gamma_{i+1}=\gamma_{i}$ ). With this extra step the detailed balance criterion for the off-diagonal entries of the transition matrix ${ }^{\mathrm{d}}$ becomes

$$
(2.11): \underbrace{P_{\mathrm{acc}}(\mu \rightarrow \nu) g(\mu \rightarrow \nu)}_{T_{\nu \mu}} p(\mu)=\underbrace{P_{\mathrm{acc}}(\nu \rightarrow \mu) g(\nu \rightarrow \mu)}_{T_{\mu \nu}} p(\nu)
$$

where $g(\mu \rightarrow \nu)$ are the probabilities that the algorithm applied on state $\mu$ proposes the new state $\nu$ (and therefore determined by the chosen modification algorithm), and $p$ is the probability distribution according to which states shall be sampled (equation 2.9). The accept ratios $P_{\text {acc }}$ are additional degrees of freedom that can be used to turn any ergodic and self-invertible algorithm for random modifications into a Markov Chain process that samples states according to a desired probability distribution. From the detailed balance criterion (2.12) it follows that the accept ratios must satisfy the relation

$$
(2.12) \Rightarrow \frac{P_{\mathrm{acc}}(\mu \rightarrow \nu)}{P_{\mathrm{acc}}(\nu \rightarrow \mu)}=\frac{g(\nu \rightarrow \mu) p(\nu)}{g(\mu \rightarrow \nu) p(\mu)}
$$

These conditions do not completely fix the accept probabilities since there is an infinite amount of solutions that satisfy $(2.12)$ and $0 \leq P_{\text {acc }}(\mu \rightarrow \nu) \leq 1$ (which accept probabilities naturally have to obey). The best choice is choosing the accept probabilities as large

\footnotetext{
${ }^{\mathrm{c}}$ Note that in the thermodynamic limit ergodicity is broken for a physical trajectory at phase coexistence.

${ }^{\mathrm{d}}$ The conditions on the diagonal entries are trivially satisfied.
} 
as possible, since that means that the amount of accepted modifications is maximized, and that the correlation between subsequent states in the generated sequence decays the fastest. This maximum value choice for the accept probabilities is

$$
P_{\text {acc }}(\mu \rightarrow \nu)=\min \left[\frac{g(\nu \rightarrow \mu) p(\nu)}{g(\mu \rightarrow \nu) p(\mu)}, 1\right] .
$$

A Markov Chain based Monte Carlo algorithm with these accept probabilities is called a Metropolis algorithm ${ }^{55}$.

\subsubsection{Single Particle Insertions and Removals}

A simple and effective choice of Monte Carlo steps for the WR model in the grand canonical ensemble is the random insertion or removal of only a single particle per step. At each step first a random species $\mathrm{X}$ is chosen with an equal probability for $\mathrm{X}=\mathrm{A}$ and $\mathrm{X}=\mathrm{B}$. Then, one chooses, again with equal probability for both possibilities, whether the step tries to insert a new particle at a random location in the volume (the generation probability for each possible microstate then is $\left.g\left(N_{\mathrm{X}} \rightarrow N_{\mathrm{X}}+1\right)=1 / V\right)$, or if a random particle of species $\mathrm{X}$ is attempted to be removed from the system $\left(g\left(N_{\mathrm{X}} \rightarrow N_{\mathrm{X}}-1\right)=1 / N_{\mathrm{X}}\right)$. It is easy to see that a simulation with this choice of steps is ergodic, since the transition between any two states is possible by first removing all particles of the original state and then sequentially inserting the particles of the other state. Detailed balance of these moves is ensured by using the Metropolis accept rates (equation 2.14). This yields the accept probabilities

$$
\begin{aligned}
P_{\text {acc }}\left(N_{\mathrm{X}} \rightarrow N_{\mathrm{X}}+1\right) & =\min \left[\frac{z_{\mathrm{X}} V}{N_{\mathrm{X}}+1}, 1\right], \text { and } \\
P_{\mathrm{acc}}\left(N_{\mathrm{X}} \rightarrow N_{\mathrm{X}}-1\right) & =\min \left[\frac{N_{\mathrm{X}}}{z_{\mathrm{X}} V}, 1\right],
\end{aligned}
$$

where $N_{\mathrm{X}}$ is the number of particles of type $\mathrm{X}$ before the Monte Carlo step. In addition to these accept probabilities, insertion steps in which the inserted particle would overlap with an existing particle of the unlike species are rejected.

While the so-constructed sequence is strongly correlated, the higher acceptance rate for each proposed change more than makes up for this. Figure 2.4 shows the average number of Monte Carlo steps $\tau$ required that a state $\gamma_{i}$ has been modified so much that $\gamma_{i+\tau}$ and $\gamma_{i}$ do not contain any common $\mathrm{A}$ or B particle, which can be considered a correlation time. Compared to the typical number of Monte Carlos steps performed in a simulation (roughly between $10^{10}$ and $10^{11}$ per simulation), the values are small, indicating that while locally the generated sequence is strongly correlated, there exists a scale smaller than the sequence length $N$ on which the elements can be considered decorrelated.

Single particle insertions and removals are a standard choice in grand canonical Monte Carlo simulations, and have been chosen in this work for their simplicity and adequate 
performance. More sophisticated "cluster moves" 56 that had originally been developed for the Asakura-Oosawa model ${ }^{57}$ (AO model) have been employed in the simulation of the Zwanzig model ${ }^{49}$, but were found to be less efficient than single particle moves: The reason those cluster moves are more efficient than single particle moves in the AO model is that they eliminate a sampling problem that is caused by the asymmetry of the particles in the AO model - a problem that does not exist in the WR model in the first place. Also, unlike "cluster flipping" moves that have been developed for the WR model ${ }^{38 ; 58}$, the single particle moves are adequate for every fugacity and easily adapted to the advanced sampling techniques introduced in chapter 4 and the presence of static obstacles (which is the focus of this thesis). 



\section{Data Analysis}

In statistical mechanics, first order phase transitions are defined in (and can only occur in) the thermodynamic limit, which implies an infinite size of the system. Real world systems are not infinite, but often still sufficiently large to be well approximated by the thermodynamic limit. The system sizes that are accessible in Monte Carlo simulations of phase transitions, however, are so small (many orders of magnitude below the $10^{23}$ particles often considered a typical thermodynamic system) that measurable deviations from the behavior in the thermodynamic limit occur. To infer the behavior of the large real world system from computer simulations, the simulation results need to be extrapolated to the thermodynamic limit. In the case of phase transitions this first requires to define analogous effects in a small system. Then, by simulating systems of different size (where each system individually is too small to represent the thermodynamic limit) the behavior in the thermodynamic limit can be estimated by extrapolation.

The WR model has been analyzed in the grand canonical ensemble and with the fluid picture in mind. This means that the order parameter characterizing the phase transition (the liquid $\leftrightarrow$ gas transition) is related to $\rho_{\mathrm{A}}$, and that $z_{\mathrm{B}}$ is considered as an inverse temperature for purposes of analysis (the model actually simulated still is the mixture). For fluids undergoing a liquid $\leftrightarrow$ gas transition, in particular for locating the critical point of the transition, a robust finite-size scaling analysis exists ${ }^{21 ; 22}$. Of course, due to the fluid $\leftrightarrow$ mixture mapping of the Widom-Rowlinson model, a phase transition in either picture automatically implies a phase transition in the other one.

\subsection{The Order Parameter Distribution}

The feature distinguishing gas and liquid is the density $\rho_{\mathrm{A}}$. Hence, the key role in all attempts to locate the liquid $\leftrightarrow$ gas transition and its critical point is played by the order parameter distribution (OPD)

$$
P\left(\rho_{\mathrm{A}} \mid z_{\mathrm{A}}, z_{\mathrm{B}}\right)
$$

which is the probability that a system of a given size $V$ is in a state that contains $N_{\mathrm{A}}=\rho_{\mathrm{A}} V$ particles of type A at fugacities $z_{\mathrm{A}}, z_{\mathrm{B}}$. The OPD allows to calculate ensemble averages $\langle\cdot\rangle$ of observables that depend on $\rho_{\mathrm{A}}$ :

$$
\left\langle f\left(\rho_{\mathrm{A}}\right)\right\rangle:=\int_{0}^{\infty} \mathrm{d} \rho_{\mathrm{A}} f\left(\rho_{\mathrm{A}}\right) P\left(\rho_{\mathrm{A}} \mid z_{\mathrm{A}}, z_{\mathrm{B}}\right)
$$


Because of the symmetry of the Widom-Rowlinson model, it is known that the phase transition from liquid to gas must occur at $z_{\mathrm{A}}=z_{\mathrm{B}}$, which leaves only a single degree of freedom (say, $z_{\mathrm{B}}$ ) for the possible location of the critical point of the pure model. OPDs can be directly extracted from Monte Carlo simulations performed at the sought-for fugacities (see chapter 2.2); more efficient methods that allow to generate OPDs for many different fugacities from a single simulation are discussed in chapter 4 .

As shown in figure 3.1, order parameter distributions for fugacities above the critical fugacity $z_{\mathrm{B}, \mathrm{cr}} \approx 1.718$ look qualitatively different from those at $z_{\mathrm{B}}<z_{\mathrm{B}, \mathrm{cr}}$. For $z_{\mathrm{B}}>z_{\mathrm{B}, \mathrm{cr}}$, the OPD features two distinct peaks: The system is either in a state of low density or a state of high density but not likely in a state of intermediate density. This situation appears to be the finite-size equivalent of a phase equilibrium that would occur in the thermodynamic limit, where liquid and gas (both with a well-defined density) are an equally likely state of the system. To reflect this, the low and high density states are referred to as pseudophases, which shall refer to anything that "looks like" it is the finite-size equivalent of a phase that exists in the thermodynamic limit ${ }^{\mathrm{a}}$. Consequently, the single peak seen for $z_{\mathrm{B}}<z_{\mathrm{B}, \mathrm{cr}}$ is attributed to the supercritical pseudophase. When $z_{\mathrm{B}}$ is increased, at the critical point the peak of the supercritical pseudophase broadens, develops to a structure of two overlapping peaks at the critical critical point (as shown in figure 3.1a), and finally results into the two non-overlapping peaks of two pseudophases at phase equilibrium. With the exception of the critical fugacity, the peaks seen in the OPD become sharper and (in case of the bimodal distribution at $z_{\mathrm{B}}>z_{\mathrm{B}, \mathrm{cr}}$ ) increasingly disconnected when the system size is increased. This indicates that in the thermodynamic limit the three pseudophases indeed become proper phases with well-defined densities (i.e. $\delta$ peaks in the OPD).

\subsubsection{Order Parameter and Analogy to the Ising Model}

The behavior of the order parameter distribution above and below the critical temperature and as a function of the system size motivates the definition of the following order parameter to distinguish the supercritical fluid state from the liquid-gas equilibrium:

$$
\langle|m|\rangle, \text { where } m:=\rho_{\mathrm{A}}-\left\langle\rho_{\mathrm{A}}\right\rangle .
$$

Depending on $z_{\mathrm{B}},\left\langle\rho_{\mathrm{A}}\right\rangle$ is either the average density of the supercritical peak (for $z_{\mathrm{B}}<z_{\mathrm{B}, \mathrm{cr}}$ ) or an average density of the gas and liquid pseudophases (for $z_{\mathrm{B}}>z_{\mathrm{B}, \mathrm{cr}}$ ). Since the peaks become sharp for $L \rightarrow \infty$ (except at the critical point) the ensemble average of $|m|$ either converges to zero (for the supercritical phase), or to a measure of the density difference of liquid and gas phase.

\footnotetext{
a I would like to point out that the term "pseudophase" is not a term used in literature, where the term "phase" is commonly used for the small systems of computer simulations, too. The reason I introduced a new term is that extrapolation to large systems can also reveal that in the thermodynamic limit the respective phase does not exist (see chapter 8).
} 

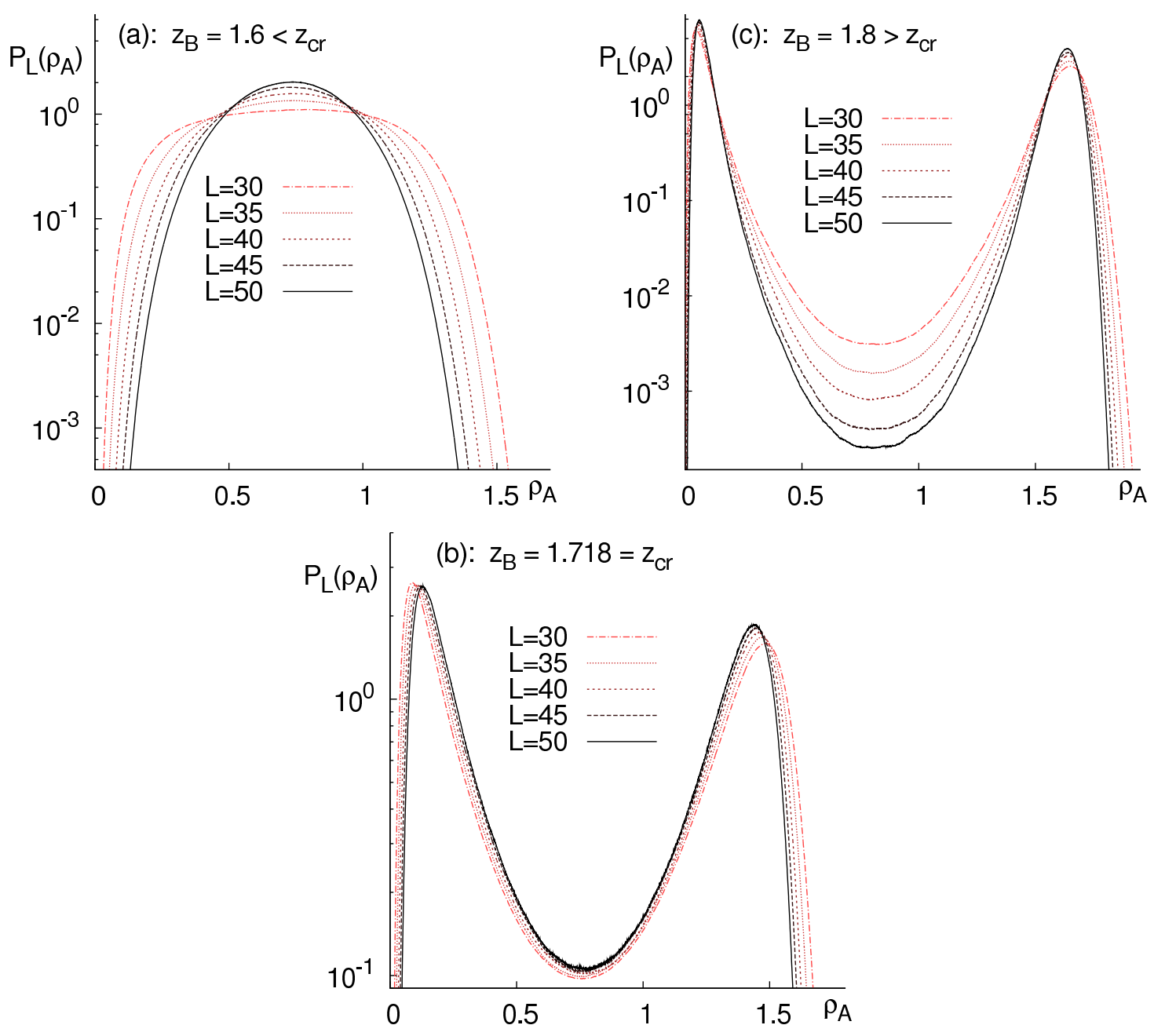

Figure 3.1: Order parameter distributions for different system sizes $L$ for a fugacity below, at, and above the critical fugacity $z_{\mathrm{B}, \mathrm{cr}}$ (figures a, b, and c, respectively). Below the critical fugacity, the OPD is a single peak that, upon increasing the system size, becomes Gaussian and sharp. Above the critical fugacity (figure c), it features a peak at low and at high density, which represent the gas and the liquid pseudophase, respectively. As the system size is increased, the two peaks become sharp and the in-between minimum approaches zero. Figure (b) shows that at the critical point the OPD assumes the shape of two overlapping peaks that only weakly depends on the system size. 


\begin{tabular}{l|llll} 
& $\alpha$ & $\beta$ & $\gamma$ & $\nu$ \\
\hline 2D Ising & 0 & $1 / 8$ & $7 / 4$ & 1 \\
3D Ising & $0.109(4)$ & $0.3258(14)$ & $1.2396(13)$ & $0.6304(13)$
\end{tabular}

Table 3.1: Critical exponents for the two- and three-dimensional Ising model. The exponents for $d=2$ are exact, owing to an exact partition function of the Ising model in two dimensions ${ }^{59}$, the $d=3$ values are estimated from renormalization group calculations ${ }^{60 ; 61}$.

The order parameter $\langle|m|\rangle$ is constructed as a direct equivalent of the magnitude of magnetization in Ising models, where the two ferromagnetic phases (spins up and spins down) are loosely identified with the liquid and the gas phase. As the equivalents of the reduced temperature $t$ and the external magnetic field $h$ of the Ising model the parameters

$$
\begin{aligned}
t & :=\frac{z_{\mathrm{B}, \mathrm{cr}}-z_{\mathrm{B}}}{z_{\mathrm{B}, \mathrm{cr}}} \\
h & :=\mu_{\mathrm{A}}-\mu_{\mathrm{A}, \mathrm{cr}}
\end{aligned}
$$

are defined, where $\mu_{\mathrm{A}, \mathrm{cr}}$ is the value of $\mu_{\mathrm{A}}$ at the critical point. The expression for $t$ follows from the interpretation of $z_{\mathrm{B}}$ as an inverse temperature, the interpretation of $\mu_{\mathrm{A}}$ as an external field is suggested by the interpretation of $\rho_{\mathrm{A}}$ being related to the magnetization.

\subsubsection{Indicators for the Critical Point}

Having defined an order parameter suggests that the location of the critical point can be found by locating the $\mathrm{B}$ fugacity where $\langle|m|\rangle$ changes from being zero to being distinctively non-zero. But as shown in figure 3.2(a), $\langle|m|\rangle$ is non-zero even far below the critical point. Also, the order parameter strongly depends on the system size. Inspired by the Ising model, generalized susceptibilities are being used to locate the critical point, instead.

For Ising models close to the critical point the derivatives of the singular part of the free energy density with respect to the external field $h$ scale as

$$
\frac{\partial^{n} f(t, h)}{\partial h^{n}}=|t|^{\beta+(1-n)(\beta+\gamma)} \cdot \text { const, }
$$

where $\beta$ and $\gamma$ are the usual critical exponents for the order parameter $\langle|m|\rangle$ and susceptibility $\chi^{(2)}$, respectively ${ }^{1}$. Since for $n \geq 2$ this implies a divergence at the critical point (cf. the critical exponents of the Ising model in table 3.1), the following analogous 

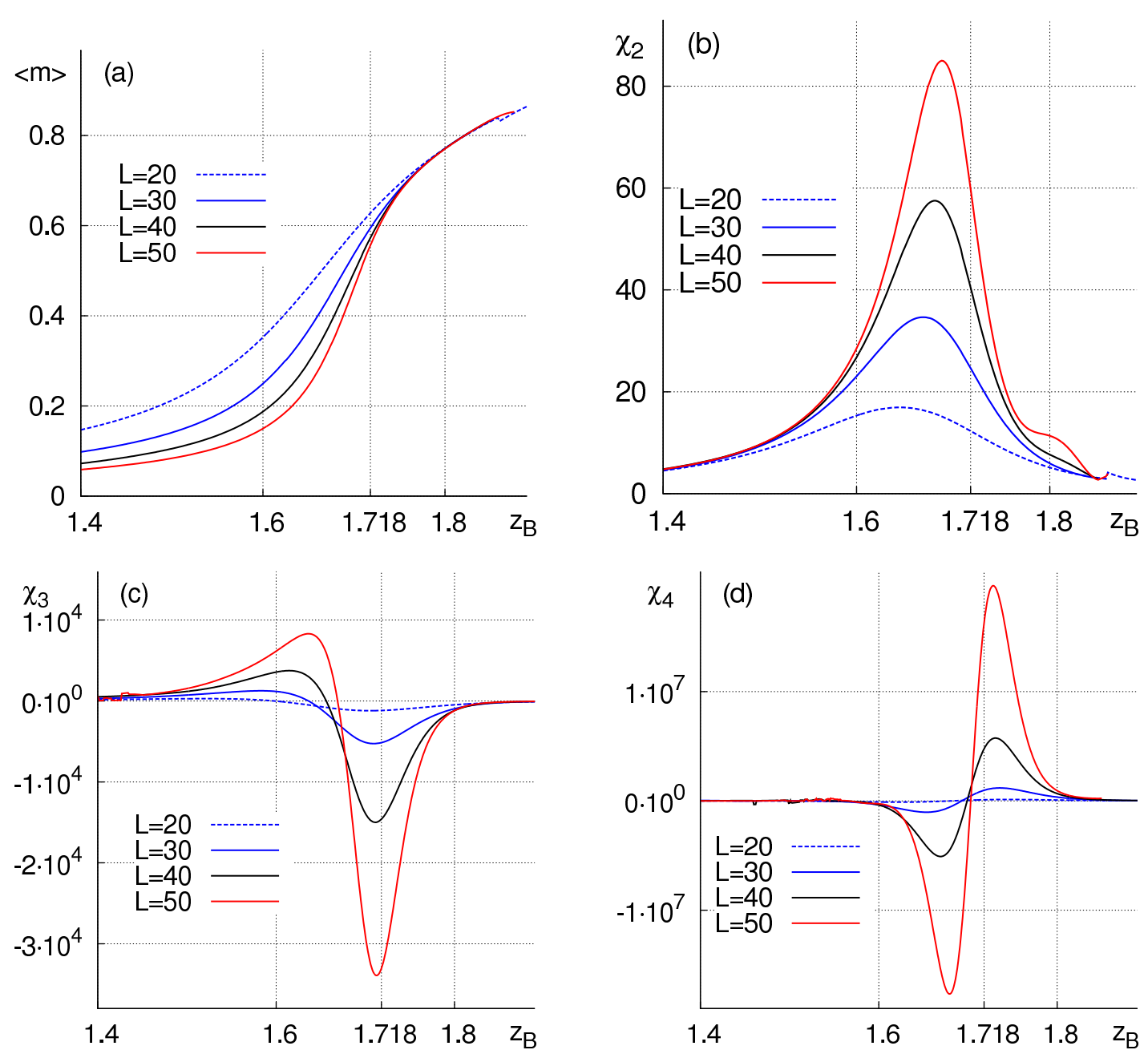

Figure 3.2: Running of different observables with inverse temperature $z_{\mathrm{B}}$ for $2 \mathrm{D}$ square WR systems of edge length $L$. Figure (a) shows the behavior of the order parameter defined in (3.3). Owing to the finite size of the systems, it is distinctively non-zero even for $z_{\mathrm{B}}<z_{\mathrm{B}, \mathrm{cr}}$, and does not indicate the critical fugacity very well. Figures (b), (c), and (d) show the functionals $\chi_{2}, \chi_{3}$, and $\chi_{4}$ (equations 3.10-3.12), which show clearly identifiable extrema in the vicinity of the critical point. With increasing system size, these extrema become more pronounced and approach the critical point. By using finite-size scaling (section 3.2), this behavior can be exploited to find the critical fugacity of the thermodynamic system. 
observables should diverge in the Widom-Rowlinson model:

$$
\begin{aligned}
\chi^{(2)} & =\left.\left.\frac{1}{V} \frac{\partial^{2} \log \mathcal{Z}}{\partial h^{2}}\right|_{t} \stackrel{(3.4,3.5)}{=} \frac{1}{V} \frac{\partial^{2} \log \mathcal{Z}}{\partial \mu_{\mathrm{A}}^{2}}\right|_{z_{\mathrm{B}}} \\
& \stackrel{(A .8)}{=} \frac{1}{V}\left\langle\left(N_{\mathrm{A}}-\left\langle N_{\mathrm{A}}\right\rangle\right)^{2}\right\rangle \\
\chi^{(3)} & =\left.\frac{1}{V} \frac{\partial^{3} \log \mathcal{Z}}{\partial \mu_{\mathrm{A}}^{3}}\right|_{z_{\mathrm{B}}} \stackrel{(A .9)}{=} \frac{1}{V}\left\langle\left(N_{\mathrm{A}}-\left\langle N_{\mathrm{A}}\right\rangle\right)^{3}\right\rangle \\
\chi^{(4)} & =\left.\frac{1}{V} \frac{\partial^{4} \log \mathcal{Z}}{\partial \mu_{\mathrm{A}}^{4}}\right|_{z_{\mathrm{B}}} \\
\stackrel{(A .10)}{=} & \frac{1}{V}\left\langle\left(N_{\mathrm{A}}-\left\langle N_{\mathrm{A}}\right\rangle\right)^{4}\right\rangle-\frac{1}{V}\left\langle\left(N_{\mathrm{A}}-\left\langle N_{\mathrm{A}}\right\rangle\right)^{2}\right\rangle^{2}
\end{aligned}
$$

\subsubsection{Coexistence-stable Estimators}

There is one problem with these generalized susceptibilities: they cannot be obtained from the order parameter distributions by the direct application of the equations (3.73.9). The reason is that the expressions (3.7-3.9) assume the existence of only a single phase, whereas order parameter distributions simultaneously contain a liquid and gas pseudophase. Particularly at the critical point, the OPD already features two overlapping peaks (cf. figure 3.6a). For bimodal order parameter distributions, the problem is resolved by considering the following functionals over the OPD, instead:

$$
\begin{aligned}
& \chi_{2}:=V\left\langle(|m|-\langle|m|\rangle)^{2}\right\rangle \\
& \chi_{3}:=V^{2}\left\langle(|m|-\langle|m|\rangle)^{3}\right\rangle \\
& \chi_{4}:=V^{3}\left[\left\langle(|m|-\langle|m|\rangle)^{4}\right\rangle-\left\langle(|m|-\langle|m|\rangle)^{2}\right\rangle^{2}\right]
\end{aligned}
$$

These functionals are the direct equivalents of those defined in (3.7-3.9), where the density $\rho_{\mathrm{A}}$ has been replaced with the magnitude of the deviation from the average density between both pseudophases. The meaning of this substitution is best understood visually: It is equivalent to mirroring the gas peak on $\left\langle\rho_{\mathrm{A}}\right\rangle$, adding it to the liquid peak, and applying the calculation rules (3.7-3.9) on this averaged peak. Since the generalized susceptibilities of both phases diverge with the same critical exponent, the averaged peak also diverges with this exponent. The functionals in (3.10-3.12) should therefore provide proper estimators of the critical point in the sense that they diverge at the critical point (except for finite-size effects that are discussed in the next section), and do so with the same exponents as the physical observables $\chi^{(2)}, \chi^{(3)}$, and $\chi^{(4)}$ would.

Using the functionals defined in (3.10-3.12) to locate the critical point solves the problem of simultaneous appearance of pseudophases in the OPD. But this raises the question how appropriate these measures are in the supercritical region where only one phase is present. If one assumes that the OPD in the supercritical region has a Gaussian shape 
(which it assumes for sufficiently large systems), then by direct calculation one can verify the relations

$$
\begin{aligned}
& \chi_{2}=\left(\frac{1}{2}-\frac{1}{\pi}\right) \chi^{(2)}, \\
& \chi_{3}=\frac{(4-\pi)}{2 \pi^{3 / 2}}\left(\chi^{(2)}\right)^{3 / 2}, \\
& \chi_{4}=\frac{\left(-12+4 \pi+3 \pi^{2}\right)}{2 \pi^{2}}\left(\chi^{(2)}\right)^{2} .
\end{aligned}
$$

Since $\chi^{(2)}$ does not diverge in the supercritical region (except when the critical point is approached), $\chi_{2}, \chi_{3}$, and $\chi_{4}$ are finite there, and diverge only at criticality.

In figure 3.2 the behavior of the functionals $\chi_{2}, \chi_{3}$ and $\chi_{4}$ is shown as a function of $z_{\mathrm{B}}$ for two-dimensional systems of different size. In all three cases, well-pronounced extrema in the vicinity of the critical point and a vanishing away from criticality are seen. The reason that rounded peaks rather than real divergences are seen and that the locations of the extrema deviate from the critical fugacity is the finite size of the systems. By using finite-size scaling ${ }^{19 ; 62}$ the location and height of the peaks can be quantitatively understood, and the critical point of the system in the thermodynamic limit (as well as the critical exponents) can be inferred.

\subsection{Finite-size Scaling at conventional Critical Points}

The key concept in finite-size scaling around critical points is the correlation length $\xi$, which can be considered the length scale over which density fluctuations in the system are correlated. If $\xi$ is much smaller than the lateral extension $L$ of a system, then the system is sufficiently large to capture the physics of the model and only small corrections due to boundary terms are expected ${ }^{23}$. On the length scale $L \gg \xi$, the system looks homogeneous and is said to be self averaging. If on the other hand $L<\xi$, then the size of the simulated system is insufficient to exhibit all the correlations that exist in the thermodynamic limit. Such a situation necessarily occurs in the proximity of the critical point, where the correlation length diverges with the positive correlation length exponent $\nu \operatorname{as}^{3}$

$$
\xi \sim|t|^{-\nu}
$$

Because of this divergence, no simulation of a finite size system can ever display the true behavior of the thermodynamic limit arbitrarily close to the critical point. Rather, the largest correlation length that can be attained in such a system is of the order of and proportional to the system's size, i.e. $\xi \sim L$ (this statement is called the finite-size scaling Ansatz). If $|t|$ in equation 3.13 is interpreted as the proximity to the critical point, then the finite-size scaling Ansatz can be expressed in the following way: The closest proximity 

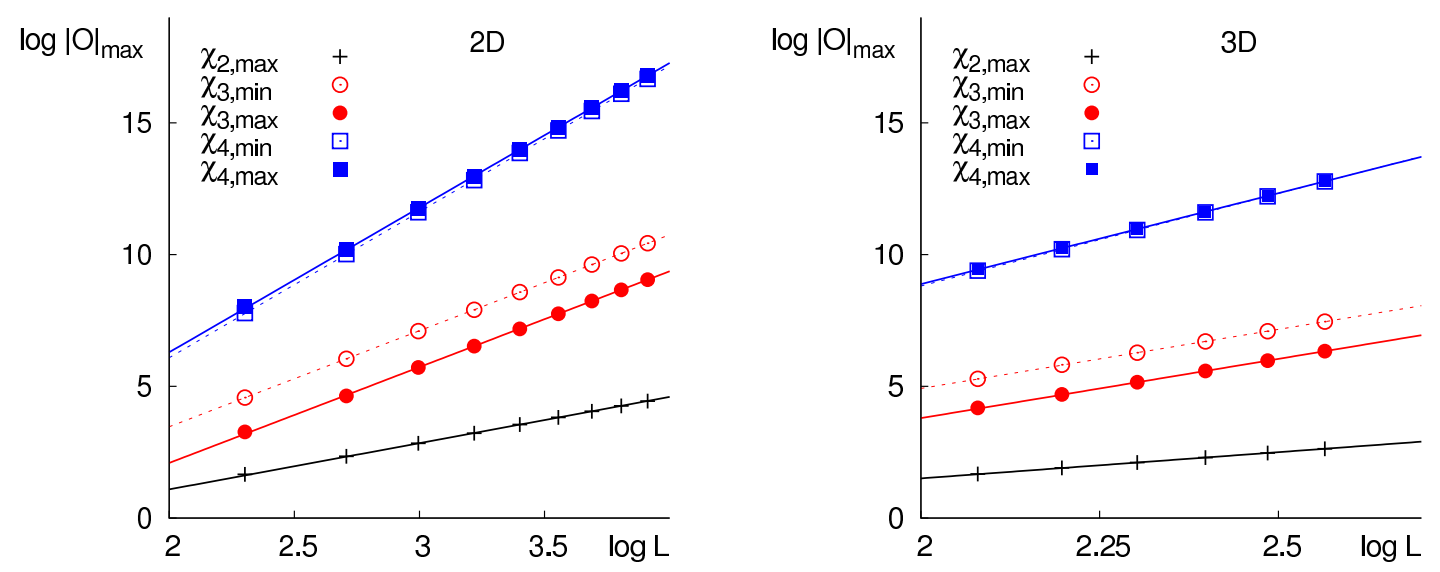

Figure 3.3: Magnitudes of the peaks in the functionals $\chi_{n}$ seen in figure 3.2 for the WR model in two and three dimensions. As a function of the system size all data are compatible with the scaling predicted in equation (3.14), as demonstrated by the linear fits on the data points (in double-logarithmic display). As shown in table 3.2, the slopes of the fits are compatible with the expectations for the observables $\chi^{(i)}$ defined in (3.7-3.9).

\begin{tabular}{l|ll|ll|ll} 
& $\chi^{(2)}$ & $\chi_{2}$ & $\chi^{(3)}$ & $\chi_{3}$ & $\chi^{(4)}$ & $\chi_{4}$ \\
\hline $2 \mathrm{D}$ & 1.75 & 1.75 & 3.625 & 3.64 & 5.5 & 5.52 \\
$3 \mathrm{D}$ & $1.966(06)$ & 1.99 & $4.449(16)$ & 4.49 & $6.932(24)$ & 6.95
\end{tabular}

Table 3.2: Exponents for the scaling of susceptibility-related observables with system size $L$ for the WR model. The exponents for the generalized susceptibilities $\chi^{(n)}$ have been calculated using the critical exponents in table 3.1. The exponents for the functionals $\chi_{n}$ have been inferred from the slopes in figure 3.3, and match that of the $\chi^{(n)}$ (which the $\chi_{n}$ were constructed to represent) well.

to criticality $|t|_{\min }$ that the system can exhibit is a function of the system size:

$$
|t|_{\min } \sim L^{-1 / \nu}
$$

This is simply a rewrite of (3.13) with the finite-size scaling Ansatz $\xi \sim L$. This maximum amount of criticality also affects the behavior of other observables in a finite system. Particularly, an observable $Y$ that diverges with a scaling law $O \sim|t|^{-w}$ close to the critical point only reaches a finite size-dependent maximum

$$
|O|_{\max } \sim|t|_{\min }^{-w} \stackrel{(3.14)}{\sim} L^{w / \nu}
$$

Relation 3.15 not only qualitatively explains the rounded peaks seen for $\chi_{2}, \chi_{3}$, and $\chi_{4}$. By comparing the values of the global extrema ${ }^{b}$ for different system sizes it also allows to

\footnotetext{
${ }^{\mathrm{b}}$ The functional $\chi_{4}$ also exhibits a third extremum that is local. It has been ignored here because of
} 
measure the exponents $w / \nu$. As shown in figure 3.3, the extrema indeed scale with the system size according to a scaling relation like (3.15), and allow to extract values for the exponents (table 3.2). Generally, this method allows to evaluate critical exponents (up to the factor $1 / \nu$ ) from computer simulation results. Here, comparison with the known critical exponents of the Ising model allows for a non-trivial test that the functionals $\chi_{n}$ (for $n=2,3,4)$ indeed are a proper representation of the generalized susceptibilities $\chi^{(n)}$ at criticality.

From equation 3.6 and relation 3.15 the generalized susceptibilities $\chi^{(2)}, \chi^{(3)}$, and $\chi^{(4)}$ are expected to scale with the system size as $L^{\gamma / \nu}, L^{(2 \gamma+\beta) / \nu}$, and $L^{(3 \gamma+2 \beta) / \nu}$, respectively. Using the values for critical exponents of the the Ising universality in table 3.1, these scaling exponents can be calculated. As shown in table 3.2, the exponents for the $\chi^{(n)}$ are equal to the exponents read off from figure 3.3 for the $\chi_{n}$. This demonstrates that the $\chi_{n}$ defined in (3.10-3.12) indeed are adequate functionals to represent the behavior of the generalized susceptibilities $\chi^{(n)}$.

\subsubsection{Pseudocritical Points}

As already seen in figure 3.2 there is also another effect of the system size on the observables near the critical point. The location of the extrema of $\chi_{2}, \chi_{3}$, and $\chi_{4}$ is not the critical point and depends on the system size. In the thermodynamic limit, the fugacities of the extrema should of course converge towards the critical fugacity. Hence, their locations are referred to as pseudocritical fugacities or pseudocritical points. In analogy to pseudophases, this term indicates a fugacity that has a characteristic that is the finite-size equivalent of a critical point's characteristic. Note that even if only one critical point exists, there can be many different pseudocritical fugacities in a finite system.

Generally, for an observable $O$ that scales like $O \sim t^{-w}$ in the thermodynamic limit its finite-size equivalent scales with the system length scale $L$ as ${ }^{53}$

$$
O(t) \sim L^{w / \nu} \bar{O}\left(t L^{1 / \nu}\right)
$$

The function $\bar{O}$ in this relation is the universal scaling function for the respective observable, which does not depend on $L$. Its extrema will generally not lie at $t L^{1 / \nu}=0$ (the extrema of $O(t)$ still converge towards $t \rightarrow 0$ as long as $L^{1 / \nu} \rightarrow \infty$ ). Hence, the pseudocritical fugacities $z_{L \text {,cr }}$ defined as the extrema of $\chi_{2}, \chi_{3}$, and $\chi_{4}$ deviate from the critical fugacity $z_{\mathrm{B}, \mathrm{cr}}$ by some amount that systematically depends on $L$. If an extremum

algorithmic problems (distinguishing a local extremum from a random fluctuation in imperfect data) and because it is not required. 

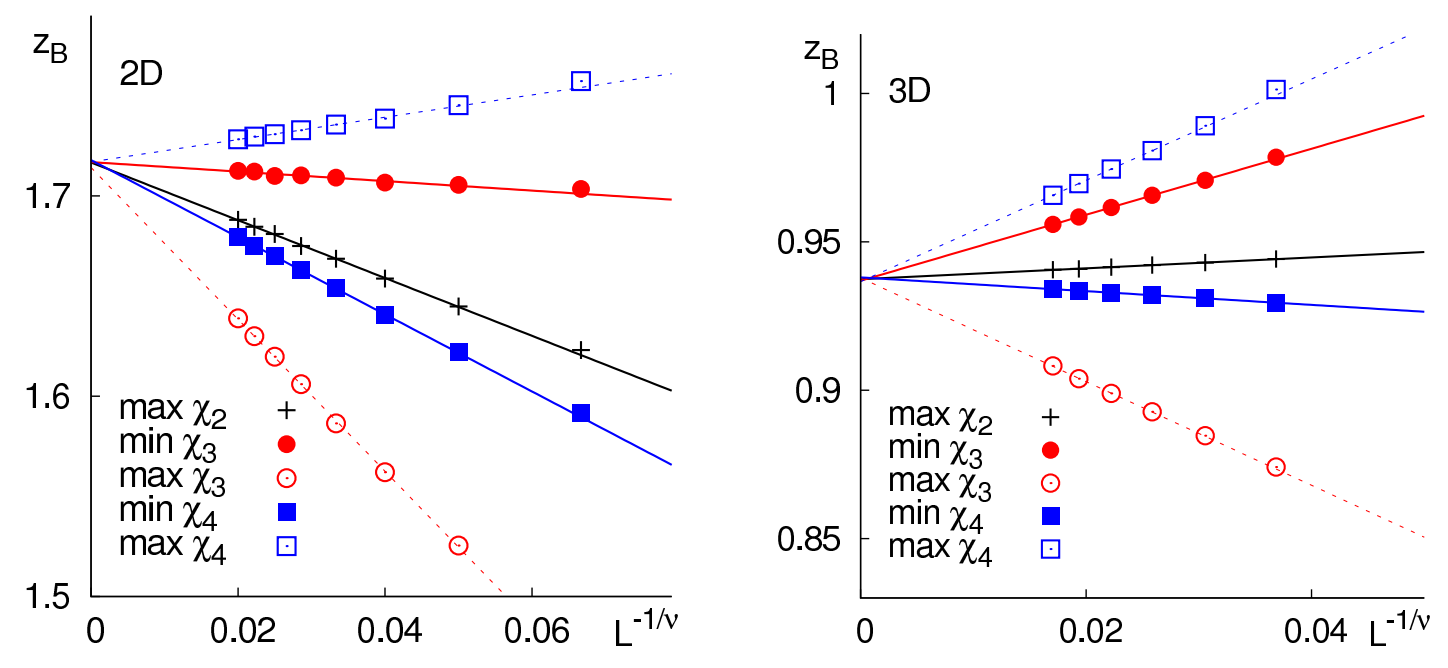

Figure 3.4: Finite-size extrapolation of pseudocritical fugacities according to (3.17) for the 2D and 3D Widom-Rowlinson model (left and right plot, respectively). As pseudocritical fugacities, the global extrema of $\chi_{2}, \chi_{3}$, and $\chi_{4}$ (equations 3.10-3.12) have been selected. For the linear fits according to (3.17) the Ising exponents $\nu=1$ (2D, exact) and $\nu=0.63$ (3D, approximate $\left.{ }^{61}\right)$ are used. In the thermodynamic limit $L^{-1 / \nu} \rightarrow 0$, all pseudocritical fugacities scale towards a single value, the critical fugacity $z_{\mathrm{B}, \mathrm{cr}}$. The results shown here predict $z_{\mathrm{B}, \mathrm{cr}}=1.7178(6)$ for the two dimensional, and $z_{\mathrm{B}, \mathrm{cr}}=0.9372(8)$ for the three dimensional WR model. In the the case shown here, where different pseudocritical points approach $z_{\mathrm{B}, \mathrm{cr}}$ from above and from below, this method for finding the critical point can be used even if $\nu$ is not known beforehand - it then becomes a fit parameter.

of $\bar{O}(x)$ lies at some $x=x_{\max }$, then

$$
\begin{aligned}
\frac{z_{\mathrm{B}, \mathrm{cr}}-z_{L, \mathrm{cr}}}{z_{\mathrm{B}, \mathrm{cr}}} L^{1 / \nu} & =x_{\max } \\
z_{\mathrm{B}, \mathrm{cr}}-z_{L, \mathrm{cr}} & =z_{\mathrm{B}, \mathrm{cr}} x_{\max } L^{-1 / \nu} \\
z_{L, \mathrm{cr}} & =z_{\mathrm{B}, \mathrm{cr}}-\operatorname{const} \cdot L^{-1 / \nu}
\end{aligned}
$$

If $\nu$ is known (at least approximately), then relation 3.17 can be used to find the critical fugacity from a set of pseudocritical fugacities for different system sizes by plotting $z_{L, \mathrm{cr}}$ over $L^{-1 / \nu}$ and linearly extrapolating to $L^{-1 / \nu} \rightarrow 0 \Leftrightarrow L \rightarrow \infty$. This is shown in figure 3.4, where the Ising model exponents $\nu=1(2 \mathrm{D})$ and $\nu \approx 0.63$ (3D) have been used. On the scale of the plot the individual linear fits converge towards a common point as $L \rightarrow \infty$, which is the finite-size corrected estimate for the critical fugacity $z_{\mathrm{B}, \mathrm{cr}}$. Conveniently, the linear fits for different pseudocritical points approach the critical fugacity from above and from below, which makes the extrapolation very reliable.

From the scatter between the different linear fits in figure 3.4 an estimate for the accuracy of $z_{\mathrm{B}, \mathrm{cr}}$ can be inferred, which is two orders of magnitude smaller than the scatter 
between the different pseudocritical points for the largest system that can be simulated. This increase of accuracy is one of the reasons for the importance of finite-size scaling in computer simulations. The other two reasons are extracting critical exponents (as demonstrated) and the possibility to distinguish finite-size equivalents of a phase transition from behavior that does not constitute a phase transition in the thermodynamic limit (some examples of this will be shown in chapter 8).

\subsubsection{Binder Cumulants}

The evaluation of $z_{\mathrm{B}, \mathrm{cr}}$ via the extrapolation of pseudocritical fugacities in (3.17) requires to either know the correlation length exponent $\nu$ beforehand, or to consider it a free parameter in the fit of the simulation data. A method that does not require an a-priori knowledge of $\nu$ is the Binder Cumulant Method ${ }^{23}$. As shown by relation 3.16, the finitesize equivalents of observables that exhibit a power law scaling of type $\sim|t|^{w}$ scale with the system length scale $L$ as proportional to $L^{-w / \nu}$. However, not all of the critical exponents are independent. Instead, scaling relations ${ }^{3}$ between them exist, such as the hyperscaling relation

$$
2-\alpha=2 \beta+\gamma=d \nu
$$

where $d$ is the spatial dimension. By making use of the hyperscaling relation, it is possible to construct a product or ratio of observables that scales with the system size as $L^{0}$, i.e. is independent of the system size at the critical point (and hopefully only there). By finding the fugacity where such an observable becomes independent of the system size, the critical fugacity can be located.

A possible construction of such a fraction is to consider the ratio of susceptibility $\chi^{(2)}$ and the order parameter $\psi$ :

$$
\frac{\chi^{(2)}}{V \psi^{2}} \sim|t|^{-\gamma} L^{-d}|t|^{2 \beta} \stackrel{(3.15)}{\sim} L^{\gamma / \nu} L^{-d} L^{2 \beta / \nu} \stackrel{(3.18)}{\sim} L^{0}
$$

where in the last step the hyperscaling relation has been used. The functionals used for describing the susceptibility and the order parameter are $\chi_{2}$ and $\langle|m|\rangle$, respectively. Therefore, a functional of the order parameter distribution that is independent of $L$ at the critical point is given by

$$
\begin{aligned}
U_{1} & :=\frac{\chi_{2}}{V\langle|m|\rangle^{2}}+1 \stackrel{(3.10)}{=} \frac{\left\langle(|m|-\langle|m|\rangle)^{2}\right\rangle}{\langle|m|\rangle^{2}}+1=\frac{\left\langle|m|^{2}\right\rangle-\langle|m|\rangle^{2}}{\langle|m|\rangle^{2}}+1 \\
& =\frac{\left\langle|m|^{2}\right\rangle}{\langle|m|\rangle^{2}} \stackrel{(3.3)}{=} \frac{\left\langle\left|\rho_{\mathrm{A}}-\left\langle\rho_{\mathrm{A}}\right\rangle\right|^{2}\right\rangle}{\left\langle\left|\rho_{\mathrm{A}}-\left\langle\rho_{\mathrm{A}}\right\rangle\right|\right\rangle^{2}} .
\end{aligned}
$$

The idea to use ratios such as $U_{1}$ to locate the critical point goes back to Kurt Binder ${ }^{23}$. In the literature, they are therefore called Binder cumulants. 

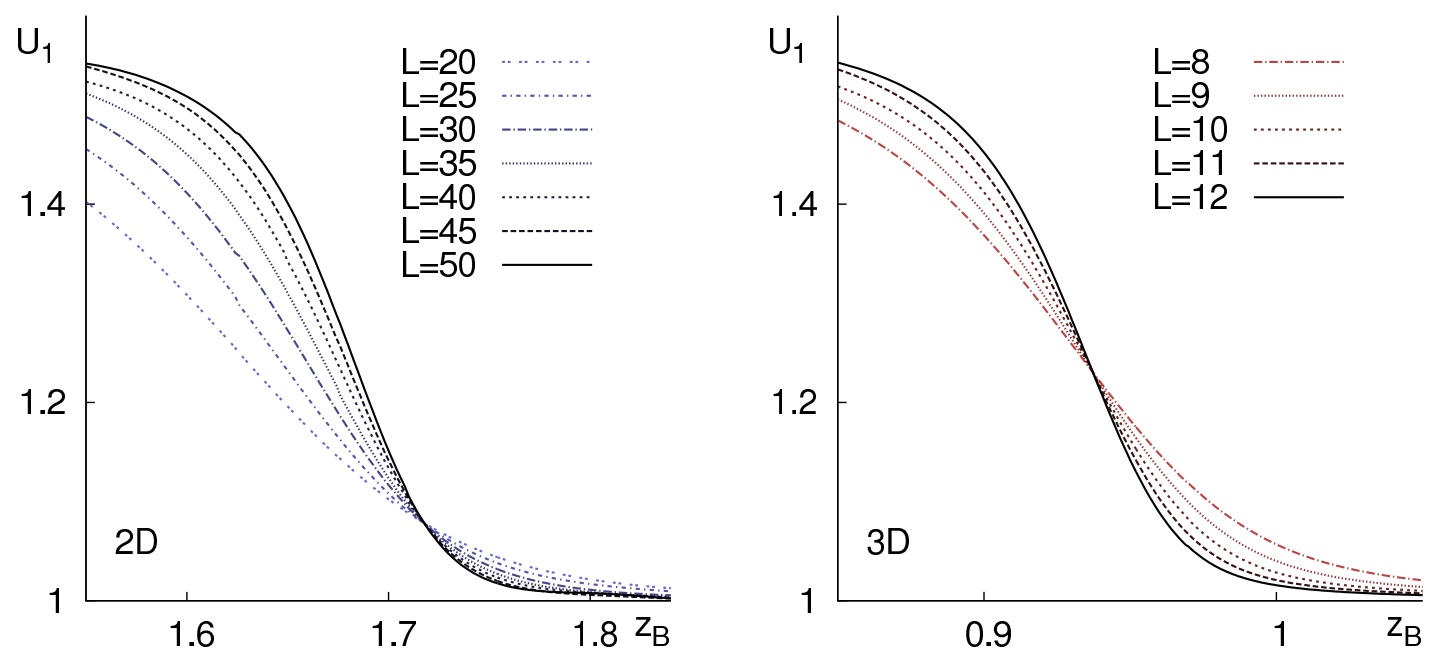

Figure 3.5: Binder cumulants $U_{1}$ for the two and three dimensional WR model. From the intersections of the cumulants for different system sizes, the critical fugacities $z_{\mathrm{B}, \mathrm{cr}}=1.718(2)$ $(2 \mathrm{D})$ and $z_{\mathrm{B}, \mathrm{cr}}=0.9374(2)(3 \mathrm{D})$ are found. The uncertainties in the estimates reflect the scatter between the curves' intersection points.

As shown in figure 3.5, the $U_{1}\left(z_{\mathrm{B}}\right)$ indeed depend on the size $L$ of a system everywhere except at one point, the critical fugacity. Hence, the method of Binder cumulants allows to evaluate $z_{\mathrm{B}, \mathrm{cr}}$ without having to know the correlation length exponent $\nu$, which makes the method convenient to use.

The shape of the $U_{1}$ curves in figure 3.5 can be understood from assuming idealized OPDs for phase equilibrium and the supercritical phase. In the thermodynamic limit, the OPD of liquid-gas equilibrium can be considered to consist of two narrow disconnected Gaussian peaks, one at liquid and one at gas density. If the width of these Gaussian peaks vanishes compared to the distance between them, $U_{1}=1$ as is easily seen from the definition in (3.20). In the supercritical region, the OPD can be considered a single narrow Gaussian. By explicit calculation one finds that in this case $U_{1}=\pi / 2$. "Intermediate" shapes, such as two overlapping peaks or an OPD consisting of three disconnected Gaussian peaks have intermediate values $1<U_{1}<\pi / 2$. Thus, the behavior of the Binder cumulant in the thermodynamic limit is

$$
\lim _{L \rightarrow \infty} U_{1}=\left\{\begin{array}{rll}
\pi / 2 & : & z_{\mathrm{B}}<z_{\mathrm{B}, \mathrm{cr}} \\
\text { const. } & : & z_{\mathrm{B}}=z_{\mathrm{B}, \mathrm{cr}} \\
1 & : & z_{\mathrm{B}}>z_{\mathrm{B}, \mathrm{cr}}
\end{array},\right.
$$

and the smooth transit from $U_{1} \approx \pi / 2$ to $U_{1} \approx 1$ should be considered a finite-size rounding effect (note that the constant that $U_{1}$ assumes at $z_{\mathrm{B}}=z_{\mathrm{B}, \text { cr }}$ is not arbitrary, but must be the same value for all system sizes by virtue of construction of $U_{1}$ ). Because of this feature, $U_{1}$ can also be used as a measure of supercriticality or phase equilibrium, 

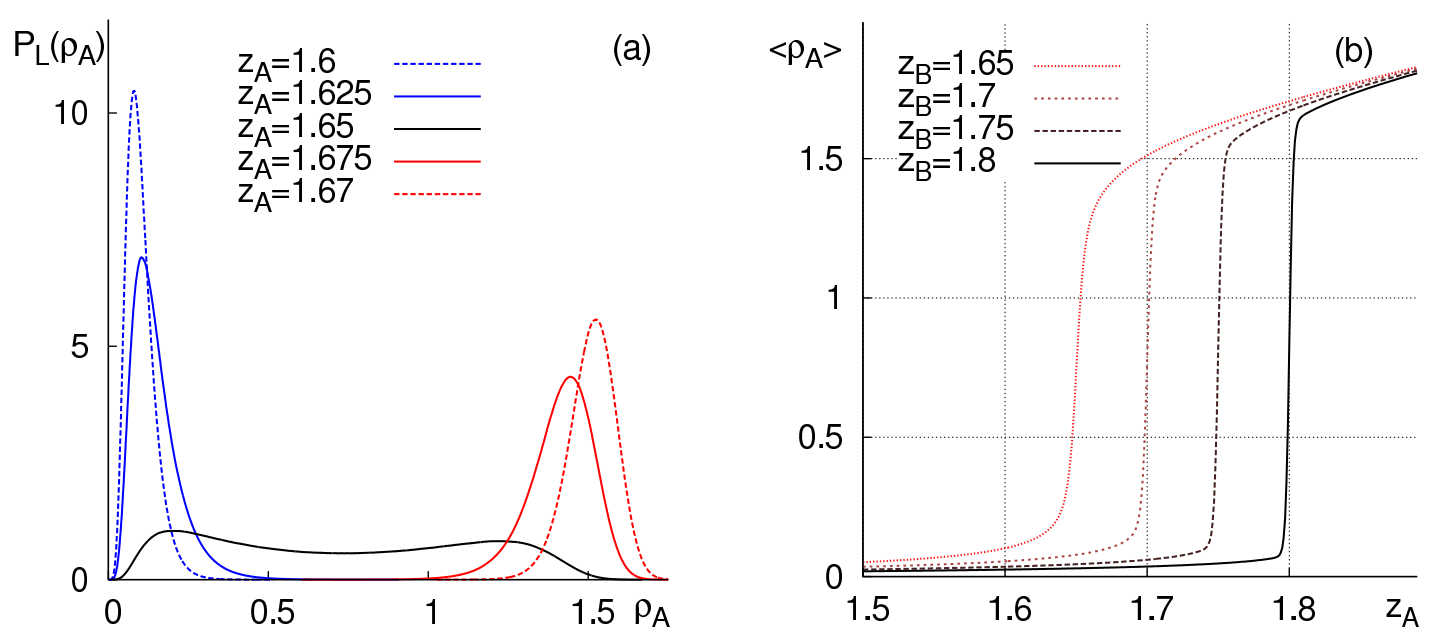

Figure 3.6: Order parameter distributions for an $L=30$ square Widom-Rowlinson system at $z_{\mathrm{B}}=1.65$, and for different $z_{\mathrm{A}}$ (figure a). As $z_{\mathrm{A}}$ is increased, the gas pseudophase develops into a liquid pseudophase via an intermediate structure of two overlapping peaks around the phase transition at $z_{\mathrm{A}}=z_{\mathrm{B}}$. In figure (b), the ensemble-averaged density is shown as a function of $z_{\mathrm{A}}$, which has its maximum slope at the symmetry line of the phase transition. This motivates the definition of the locus $l_{\mathrm{MS}}$ in $(3.22)$.

where $U_{1} \approx 1$ is interpreted as the equilibrium of two pseudophases, and $U_{1} \approx \pi / 2$ as the existence of only a single pseudophase (the supercritical one). Note that the independence of $U_{1}$ of the system size requires that hyperscaling is valid, but that the value of $U_{1}$ gives an impression of the OPD's shape, regardless. The case where hyperscaling is violated and methods to locate the critical point and quantify the violation of hyperscaling constitute a main new result of this thesis (see chapter 7).

\subsection{Constructing Loci: How to chose $z_{\mathrm{A}}$}

In the pure Widom-Rowlinson model, the symmetry between A and B particles can be used to reduce the degrees of freedom for the possible location of the critical point and the phase transition curve to one, it has to lie on the equal fugacity line $z_{\mathrm{A}}=z_{\mathrm{B}}$. In the presence of obstacles (or in other fluid models), such a symmetry may be absent. To eliminate additional degrees of freedom it has been suggested ${ }^{21 ; 22}$ to use suitably-defined loci, which are constraints that define curves $z_{\mathrm{A}}\left(z_{\mathrm{B}}\right)$ such that the curves converge towards the phase boundary as the system size is increased. Loci do not necessarily need to be an explicit mathematical expression (like $z_{\mathrm{A}}=z_{\mathrm{B}}$, which in fact is a very suitable locus for the pure WR model), but can also be defined as a condition on the OPD. That is, one chooses a suitable criterion that the OPD has to fulfill such that a) for each value of the inverse temperature $z_{\mathrm{B}}$ a unique $z_{\mathrm{A}}$ is chosen, and b) the so-created curve $z_{\mathrm{A}}=l\left(z_{\mathrm{B}}\right)$ 
converges towards and covers the phase transition curve in the thermodynamic limit ${ }^{21 ; 22}$. In figure 3.6(a) order parameter distributions for different $z_{\mathrm{A}}$ at the same inverse temperature are shown. When $z_{\mathrm{A}}$ is below the phase equilibrium value (which in this case is $z_{\mathrm{A}}=z_{\mathrm{B}}$ ), a gas peak at low density is seen, that slowly shifts towards higher densities as $z_{\mathrm{A}}$ is increased. At $z_{\mathrm{A}} \approx z_{\mathrm{B}}$, this behavior changes qualitatively, and a second peak, the liquid peak appears. Upon further increase of $z_{\mathrm{A}}$ the liquid peak grows at the expense of the gas peak until only the liquid peak remains. For even larger $z_{\mathrm{A}}$, the liquid peak then shifts to higher $\mathrm{A}$ densities. The region that the $z_{\mathrm{A}}$ should be tuned to is the region in which the liquid and the gas peak are both present in the OPD. There are different criteria that achieve this goal: The possibly most natural and most physical choice is the equal area locus $l_{\mathrm{EA}}$, which is the condition that for a given $z_{\mathrm{B}}, z_{\mathrm{A}}$ is chosen such that two peaks with an equal integrated volume exist in the OPD. Physically, this corresponds to the statement that the system is equally likely to be in the gas or in the liquid pseudophase, which clearly is a suitable criterion to define the finite-size equivalent of phase equilibrium.

While $l_{\mathrm{EA}}$ is a popular choice in Monte Carlo simulations, it is not very suitable here, because identifying two peaks fails for the supercritical region and for some of the "exotic" OPDs that can be encountered in the presence of static obstacles (e.g. those shown in figure 7.2a). A more stable choice of the locus is the maximum slope locus

$$
l_{\mathrm{MS}}:\left.\frac{\partial\left\langle\rho_{\mathrm{A}}\right\rangle}{\partial \mu_{\mathrm{A}}}\right|_{z_{\mathrm{B}}} \rightarrow \max .
$$

This locus is also well motivated by the shapes of the order parameter distribution shown in figure 3.6. Around the phase transition, the shape of the OPD quickly changes from the gas to the liquid peak. Consequently, the change on average density $\left\langle\rho_{\mathrm{A}}\right\rangle$ associated with this change of shape is large. This is shown in figure 3.6(b), which also shows that for the WR model without any obstacles the maximum slope indeed occurs at the phase equilibrium line $z_{\mathrm{A}}=z_{\mathrm{B}}$. For $z_{\mathrm{B}}$ so far below $z_{\mathrm{B}, \text { cr }}$ that only a single supercritical peak appears in the OPD, the $l_{\mathrm{MS}}$ locus still is stable, and selects $z_{\mathrm{A}}$ such that the peak has a maximum width (which in the pure model also occurs at $z_{\mathrm{A}}=z_{\mathrm{B}}$ ). 
Chapter 4

\section{Data Extrapolation}

In the previous chapters, the basics of Monte Carlo simulation and the data analysis for the identification and location of a liquid-gas type phase transition has been discussed. There is still one concept left that is required to perform a reliable simulation and analysis in a reasonable amount of CPU time, namely the extrapolation of simulation results to other thermodynamic parameters. A simulation performed at fugacities $z_{\mathrm{A}}, z_{\mathrm{B}}$ also contains information about the system at other, nearby, fugacities $\bar{z}_{\mathrm{A}}, \bar{z}_{\mathrm{B}}$ (as will be shown). This means that it is not required to perform a separate simulation for all fugacities, but that a few simulations, possibly just a single one, already allow to scan a large range of the parameter space.

\subsection{The Histogram Method}

\section{Reweighting in $z_{\mathrm{A}}$}

The central object being sampled during the Monte Carlo simulation and used in the analysis is the order parameter distribution (OPD) $P\left(N_{\mathrm{A}}\right)$, which can be expressed as

$$
P\left(N_{\mathrm{A}} \mid \mu_{\mathrm{A}}, \mu_{\mathrm{B}}\right) \stackrel{(2.8)}{=} \frac{e^{\mu_{\mathrm{A}} N_{\mathrm{A}}} Z\left(N_{\mathrm{A}}, \mu_{\mathrm{B}}\right)}{\mathcal{Z}\left(\mu_{\mathrm{A}}, \mu_{\mathrm{B}}\right)},
$$

where $Z$ and $\mathcal{Z}$ are the canonical and grand canonical partition functions of the WR model that were introduced in chapter 2. Obtaining a probability distribution $P\left(N_{\mathrm{A}}\right)$ from a Monte Carlo simulation therefore automatically generates information about the canonical partition functions. In particular, knowing the OPD fixes the ratios $Z\left(N_{\mathrm{A}}=i, \mu_{\mathrm{B}}\right) / Z\left(N_{\mathrm{A}}=j, \mu_{\mathrm{B}}\right)$ for any $i$ and $j$, as

$$
\frac{Z\left(i, \mu_{\mathrm{B}}\right)}{Z\left(j, \mu_{\mathrm{B}}\right)} \stackrel{(4.1)}{=} \frac{e^{-\mu_{\mathrm{A}} i} P\left(i \mid \mu_{\mathrm{A}}, \mu_{\mathrm{B}}\right)}{e^{-\mu_{\mathrm{A}} j} P\left(j \mid \mu_{\mathrm{A}}, \mu_{\mathrm{B}}\right)} .
$$

Conversely, knowing the canonical partition functions up to a common multiplicative constant is equivalent to knowing the order parameter distribution for every possible value of $\mu_{\mathrm{A}}$. This is, in essence, the idea behind the histogram method ${ }^{63}$ (or histogram reweighting). 

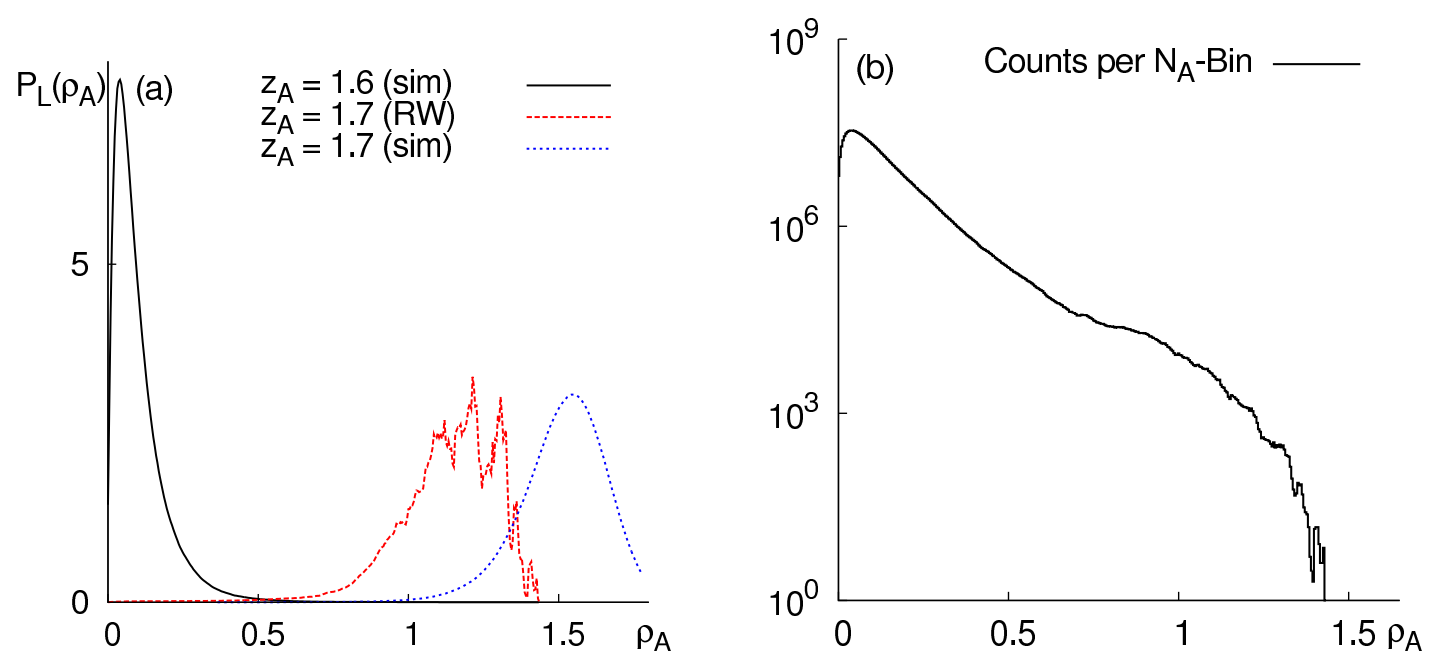

Figure 4.1: Reweighting of simulation data via equation 4.2 (figure a). Raw data are obtained from a MC simulation sampling states according to the Boltzmann distribution at $z_{\mathrm{A}}=1.6, z_{\mathrm{B}}=1.65$, which results in the OPD shown as a solid black curve. A reweighting of this OPD to $z_{\mathrm{A}}=1.7$ results in the dashed red curve, which is an incorrect result (as shown by comparison with the dashed blue curve that is the result of a direct simulation at $z_{\mathrm{A}}=1.7, z_{\mathrm{B}}=1.65$ ). The cause of this error is shown in figure (b), where the amount of generated states is plotted over $\rho_{\mathrm{A}}$ : The simulation did sample (almost) no states with $\rho_{\mathrm{A}} \approx 1.5$, which are the physically relevant densities for $z_{\mathrm{A}}=1.7$, and hence cannot predict the $\mathrm{OPD}$ in this region. 
The extrapolation of an order parameter distribution simulated at $\left(\mu_{\mathrm{A}}, \mu_{\mathrm{B}}\right)$ to $\left(\bar{\mu}_{\mathrm{A}}, \mu_{\mathrm{B}}\right)$ is easily calculated by repeated use of (4.1). This leads to the simple reweighting equation

$$
\begin{aligned}
P\left(N_{\mathrm{A}} \mid \bar{\mu}_{\mathrm{A}}, \mu_{\mathrm{B}}\right) & \stackrel{(4.1)}{=} Z\left(N_{\mathrm{A}}, \mu_{\mathrm{B}}\right) \frac{e^{\bar{\mu}_{\mathrm{A}} N_{\mathrm{A}}}}{\mathcal{Z}\left(\bar{\mu}_{\mathrm{A}}, \mu_{\mathrm{B}}\right)} \\
& \stackrel{(4.1)}{=} \frac{P\left(N_{\mathrm{A}} \mid \mu_{\mathrm{A}}, \mu_{\mathrm{B}}\right) \mathcal{Z}\left(\mu_{\mathrm{A}}, \mu_{\mathrm{B}}\right)}{e^{\mu_{\mathrm{A}} N_{\mathrm{A}}}} \frac{e^{\bar{\mu}_{\mathrm{A}} N_{\mathrm{A}}}}{\mathcal{Z}\left(\bar{\mu}_{\mathrm{A}}, \mu_{\mathrm{B}}\right)} \\
& =P\left(N_{\mathrm{A}} \mid \mu_{\mathrm{A}}, \mu_{\mathrm{B}}\right) e^{\left(\bar{\mu}_{\mathrm{A}}-\mu_{\mathrm{A}}\right) N_{\mathrm{A}}} \underbrace{\frac{\mathcal{Z}\left(\mu_{\mathrm{A}}, \mu_{\mathrm{B}}\right)}{\mathcal{Z}\left(\bar{\mu}_{\mathrm{A}}, \mu_{\mathrm{B}}\right)}}_{\text {normalization const. }}
\end{aligned}
$$

where the ratio of the grand partition functions that appears is just a constant that is fixed by the normalization of the probability distributions. In principle, equation 4.2 allows to construct the order parameter distribution for arbitrary values of $z_{\mathrm{A}}$ from just a single simulation. In practice, this does not always work, as shown in figure 4.1(a) on the example of a simulation at $z_{\mathrm{A}}=1.6, z_{\mathrm{B}}=1.65$ being reweighted to $z_{\mathrm{A}}=1.7$ (corresponding to a reweighting from a gas pseudophase to a liquid pseudophase). The reweighted OPD not only is of bad quality, but it also predicts an incorrect liquid density.

The failure of reweighting is caused by the necessarily finite number of Monte Carlo steps and the first order phase transition from gas to liquid. Since the sequence of states in the simulation is generated according to the Boltzmann distribution, the simulation generates states that almost all have a gas density. Consequently, little or no information is obtained about the $Z\left(N_{\mathrm{A}}, \mu_{\mathrm{B}}\right)$ for liquid densities, and an OPD reweighted to the point where those regions become important will have a very bad statistical accuracy - to the point of being completely wrong! Since in all analyses, the OPDs of interest are those in which the liquid and the gas pseudophase coexist, the Monte Carlo simulation introduced in section 2.2.2.1 must be modified such that good statistical accuracy is obtained over all potentially relevant $\rho_{\mathrm{A}}$. Ideally, this means to define a density interval $\left[0 ; \rho_{\mathrm{A}, \max }\right]^{\mathrm{a}}$, and have the simulation return an OPD with equally good statistics over the whole interval.

\subsection{Uniform Sampling}

A homogeneous sampling that still allows to extract the OPD from the generated Monte Carlo sequence can be achieved by sampling according to a modified Boltzmann distribution (cf. equation 2.9)

$$
p(\gamma) \sim \frac{X(\gamma)}{N_{\mathrm{A}} ! N_{\mathrm{B}} !} z_{\mathrm{A}}^{N_{\mathrm{A}}} z_{\mathrm{B}}^{N_{\mathrm{B}}} e^{w\left(N_{\mathrm{A}}\right)}
$$

\footnotetext{
${ }^{a} \mathrm{~A}$ suitable upper limit $\rho_{\mathrm{A}, \max }$ is given by the fact that the liquid densities can never exceed the densities of an ideal gas of $\mathrm{A}$ particles at the same $z_{\mathrm{A}}$.
} 
with properly chosen sampling weights $w\left(N_{\mathrm{A}}\right)^{64}$. If $C\left(N_{\mathrm{A}} \mid z_{\mathrm{A}}, z_{\mathrm{B}}\right)$ is the number of times that such a simulation sampled a state with $N_{\mathrm{A}}$ particles of species $\mathrm{A}$, then the order parameter constructed from this simulation is

$$
P\left(N_{\mathrm{A}} \mid z_{\mathrm{A}}, z_{\mathrm{B}}\right)=C\left(N_{\mathrm{A}} \mid z_{\mathrm{A}}, z_{\mathrm{B}}\right) e^{-w\left(N_{\mathrm{A}}\right)} \cdot \text { const. }
$$

Adapting the single particle insertions and removals introduced in section 2.2.2 to such a weighted simulation is simple, and only the accept probabilities for the insertion or the removal of an A particle have to be modified. The full set of accept probabilities becomes

$$
\begin{aligned}
P_{\text {acc }}\left(N_{\mathrm{A}} \rightarrow N_{\mathrm{A}}+1\right) & =\min \left[\frac{z_{\mathrm{A}} V}{N_{\mathrm{A}}+1} e^{w\left(N_{\mathrm{A}}+1\right)-w\left(N_{\mathrm{A}}\right)}, 1\right], \\
P_{\mathrm{acc}}\left(N_{\mathrm{B}} \rightarrow N_{\mathrm{B}}+1\right) & =\min \left[\frac{z_{\mathrm{B}} V}{N_{\mathrm{B}}+1}, 1\right], \\
P_{\mathrm{acc}}\left(N_{\mathrm{A}} \rightarrow N_{\mathrm{A}}-1\right) & =\min \left[\frac{N_{\mathrm{A}}}{z_{\mathrm{A}} V} e^{w\left(N_{\mathrm{A}}-1\right)-w\left(N_{\mathrm{A}}\right)}, 1\right], \\
P_{\mathrm{acc}}\left(N_{\mathrm{B}} \rightarrow N_{\mathrm{B}}-1\right) & =\min \left[\frac{N_{\mathrm{B}}}{z_{\mathrm{B}} V}, 1\right],
\end{aligned}
$$

where the extra terms caused by the sampling weights are indicated in gray. Particle numbers again refer to the initial state of the MC step.

From equation 4.4 it is apparent that the sampling becomes uniform over $N_{\mathrm{A}}$ when the weights are chosen such that

$$
w\left(N_{\mathrm{A}}\right)=-\log P\left(N_{\mathrm{A}} \mid z_{\mathrm{A}}, z_{\mathrm{B}}\right)+\text { const. }
$$

Unfortunately, (4.9) does not state how the sampling weights should be chosen, since the sought-for order parameter distribution appears on the right-hand side. Rather, the equation says that knowing a good set of weights is equivalent to knowing the order parameter distribution. The following discussion focuses on how to find a set of optimum weights, since this is the easier language to formulate the sampling strategies in.

\subsubsection{Wang-Landau Sampling}

The probably most widely used sampling strategy in current simulations are variants of Wang-Landau sampling ${ }^{65}$ (WL sampling). The original version described in the following takes an arbitrary starting choice of weights (in this case $w\left(N_{\mathrm{A}}\right)=0$ for all $N_{\mathrm{A}}$ ) and modifies it during the simulation to return an improved guess. This is repeated until some convergence criterion is met.

The process of improving the weight guesses is split into different iterations which perform successively finer modifications on the weights. During an iteration, the normal Monte Carlo moves with the current weights are performed. But after each step the weight for 
the current state is reduced by a constant amount $0<\delta \leq 1^{65}$, i.e. $w\left(N_{\mathrm{A}}\right) \rightarrow w\left(N_{\mathrm{A}}\right)-\delta$. This reflects the idea that the current density was just found to be a bit more probable than expected previously, and that therefore less weight is needed for this state. The sampling is continued until all possible values for $N_{\mathrm{A}}$ have been visited sufficiently often. A simple criterion for this is keeping track of the visited $N_{\mathrm{A}}$ values in form of a histogram $h\left(N_{\mathrm{A}}\right)$ and demanding that this histogram has become "flat" by some criterion. Here, the criterion being used is that the bin with the least counts should have at least $80 \%$ the counts of the bin with the most counts.

A full Wang-Landau simulation consists of several iterations, perhaps 10 to 30 of them. Initial iterations are performed with larger $\delta$ to quickly determine the crude shape of the best-weight curve. In subsequent runs $\delta$ is then lowered after each iteration. Later iterations therefore allow finer resolutions of the weight differences (note that the difference in weights after e.g. the first iteration can only be multiples of $\delta$, whereas the real weight differences are not quantized).

While the most simple version of Wang-Landau sampling is easy to implement and works for many systems, there are two problems associated with it: First, due to the constant adjustment of the weights, detailed balance is violated at each step. This problem is usually considered irrelevant in practice, since especially in later iterations, the modification term $\delta$, and thus the violation of detailed balance, becomes small ${ }^{65}$. The other problem is that because of the decrease $\delta \rightarrow 0$ states generated in later iterations of the sampling contribute very little to the overall result. In fact, the naive implementation has been shown to reach a saturation point beyond which further iterations do not reduce the statistical error any more ${ }^{66}$. This problem can be circumvented by using standard WL sampling as a guide for the random walk in phase space, but use different methods (than equation 4.9) to construct the $\mathrm{OPD}^{66 ; 67 ; 68}$. In this work, Wang-Landau sampling is only used in tests of the quality of a random walk in phase space (chapter 5), for which its basic version suffices. For obtaining physical results, a different uniform sampling strategy has been employed.

\subsubsection{Successive Umbrella Sampling}

Another way to achieve a roughly uniform sampling over $\rho_{\mathrm{A}}$ is to employ umbrella sampling techniques ${ }^{69}$ : Instead of sampling over the whole range of A densities, only small and overlapping sub-intervals are sampled. The full order parameter distribution can then be constructed by "gluing" together the individual results in their overlapping regions. A simulation can be restricted to a subinterval by either the choice of appropriate sampling weights or by simply rejecting all MC moves that would leave the subinterval (which is the method used in the following).

It is convenient to chose the intervals such that their size is minimal and to sample the intervals in their logical order. This is a special flavor of umbrella sampling, called 
successive umbrella sampling (SUS) ${ }^{70}$. For the WR mixture this means choosing intervals $I_{n}: N_{\mathrm{A}} \in\{n ; n+1\}$ and sampling them in the order $I_{0}, I_{1}, \ldots$ This consecutive sampling order has the advantage that the final state after simulation of interval $I_{n}$ already is close to an equilibrated state for the next interval $I_{n+1}$. Instead of having to worry about equilibrating a proper initial state for $I_{n+1}$ from scratch one can therefore take the final state of the previous interval, and merely perform a few "relaxation steps" 70 . In what follows, the additive degree of freedom in the weights in (4.9) has been fixed by the choice $w(0)=0$.

\section{Simulating the Subintervals}

At the time that the interval $I_{n}$ is being simulated, predictions of the optimum weights $w(1), w(2), \ldots, w(n)$ are already determined by simulation of the previous intervals, but $w(n+1)$ is not. For simulating $I_{n}$ some choice for $w(n+1)$ has to be made and used during the sampling. This sampling weight that is used for the accept rates of the Monte Carlo moves (equations 4.5 to 4.8 ) shall be referred to as $\widetilde{w}(n+1)$, to distinguish it from the sampling result $w(n+1)$ that is used to construct the OPD. The optimum efficiency for the simulation is obtained when $\widetilde{w}(n+1)$ is chosen such that the simulation samples an equal amount of states with $N_{\mathrm{A}}=n$ and $N_{\mathrm{A}}=n+1$, but this value is again not known prior to the simulation. Good results are achieved by linear extrapolation of the previous weights (the choice of $\widetilde{w}(1)$ is discussed below).

The simulation of $I_{n}$ is performed with the normal (biased) Monte Carlo steps, but with the additional rule that proposed states that do not lie inside the interval are always rejected. Two counters $C_{n}^{+}$and $C_{n}^{-}$keep track of how often the system ended up in a state in the upper $\left(N_{\mathrm{A}}=n+1\right)$ and the lower bin $\left(N_{\mathrm{A}}=n\right)$ of the interval, respectively. The counters are updated after every attempted Monte Carlo step, irrespective of whether the proposed step was accepted or not ${ }^{70 ; 71}$. When both counters exceed a certain number, the simulation of $I_{n}$ is terminated. The optimum weight $w(n+1)$ is then chosen as the sampling weight that would have made both counters equal:

$$
w(n+1)=\widetilde{w}(n+1)-\log \frac{C_{i}^{+}}{C_{i}^{-}}
$$

By sampling all of the intervals $I_{0}, I_{1}, \ldots$, and with the boundary condition $w(0)=0$, all "optimum weights" $w(0), w(1), \ldots$ are determined, from which the order parameter distribution can be constructed via use of equation (4.9):

$$
P\left(N_{\mathrm{A}} \mid z_{\mathrm{A}}, z_{\mathrm{B}}\right) \stackrel{(4.9)}{=} \text { const. } \cdot e^{w\left(N_{\mathrm{A}}\right)}
$$




\section{Optimizing the first Interval}

The only interval that does not benefit from the simulation of previous intervals is the first, $I_{0}$. But in case of the Widom-Rowlinson model, an equilibrated starting state and the optimum sampling weight $w(1)$ can be explicitly constructed. The generation of an equilibrated state is straightforward, since in the interval $I_{0}$ there is at most one A particle in the system. If there is no A particle in the system at all, then the B particles form an ideal gas. This means that $N_{\mathrm{B}}$ is a random variable from a Poissonian distribution with mean $z_{\mathrm{B}} V$, and that the locations of the $\mathrm{B}$ particles are completely uncorrelated. Thus, by drawing a random $N_{\mathrm{B}}$ from this Poissonian and adding this amount of B particles into the system at random locations, an equilibrated state for the ensemble with $N_{\mathrm{A}}=0$ is created. By performing a few thousand $\mathrm{MC}$ steps in window $I_{0}$ before starting to count $C_{0}^{+}$and $C_{0}^{-}$an equilibrated initial state is generated.

The optimum weight $w(1)$, and therefore the optimum choice for $\widetilde{w}(1)$, can be calculated explicitly: For $N_{\mathrm{A}}=0$, the $\mathrm{B}$ particles form an ideal gas in the system volume. The microcanonical partition function is

$$
\Omega\left(V, 0, N_{\mathrm{B}}\right)=\frac{1}{N_{\mathrm{B}} ! 0 !} \int_{V} \mathrm{~d}^{d N_{\mathrm{B}}} x 1=\frac{V^{N_{\mathrm{B}}}}{N_{\mathrm{B}} !} .
$$

If $N_{\mathrm{A}}=1$, the $\mathrm{B}$ particles are an ideal gas is the remaining volume $V-V_{X}$, where $V_{X}$ is the volume that the A particle excludes for the B particles (which is the volume of a $d$-dimensional unit sphere). The partition function is

$$
\Omega\left(V, 1, N_{\mathrm{B}}\right)=\frac{1}{N_{\mathrm{B}} ! 1 !} \underbrace{\int_{V-V_{X}} \mathrm{~d}^{d N_{\mathrm{B}}} y}_{\text {B particles }} \underbrace{\int_{V} \mathrm{~d}^{d} x}_{\text {A particle }} 1=\frac{V\left(V-V_{X}\right)^{N_{\mathrm{B}}}}{N_{\mathrm{B}} !} .
$$

It follows that the ideal choice for $\widetilde{w}(1)$ is

$$
\begin{aligned}
& w(1)-\underbrace{w(0)}_{=0} \quad=\quad \log P_{L}\left(N_{\mathrm{A}}=1\right)-\log P_{L}\left(N_{\mathrm{A}}=0\right) \\
& \stackrel{(4.1)}{=} \quad \log \frac{e^{\mu_{\mathrm{A}}} Z\left(1, \mu_{\mathrm{B}}\right)}{Z\left(0, \mu_{\mathrm{B}}\right)} \\
& =\quad \log \frac{\sum_{N_{\mathrm{B}}=0}^{\infty} z_{\mathrm{A}}^{1} z_{\mathrm{B}}^{N_{\mathrm{B}}} \Omega\left(V, 1, N_{\mathrm{B}}\right)}{\sum_{N_{\mathrm{B}}=0}^{\infty} z_{\mathrm{A}}^{0} z_{\mathrm{B}}^{N_{\mathrm{B}}} \Omega\left(V, 0, N_{\mathrm{B}}\right)} \\
& \stackrel{(4.12,4.13)}{=} \log \frac{z_{\mathrm{A}} \sum_{N_{\mathrm{B}}=0}^{\infty} z_{\mathrm{B}}^{N_{\mathrm{B}}} \frac{V\left(V-V_{X}\right)^{N_{\mathrm{B}}}}{N_{\mathrm{B}} !}}{\sum_{N_{\mathrm{B}}=0}^{\infty} z_{\mathrm{B}}^{N_{\mathrm{B}}} \frac{V^{N_{\mathrm{B}}}}{N_{\mathrm{B}} !}} \\
& w(1)=\quad \log \frac{z_{\mathrm{A}} V e^{z_{\mathrm{B}}\left(V-V_{X}\right)}}{e^{z_{\mathrm{B}} V}}=-z_{\mathrm{B}} V_{X}+\log \left[z_{\mathrm{A}} V\right] .
\end{aligned}
$$


The solution in equation 4.14 only applies to the first sampling interval, and only in the case that no static obstacles are present in the system. In the presence of a low amount of static obstacles (which is the case that is discussed here) the exact solution for the pure model may still be an adequate choice for the sampling weight in $I_{0}$. In fact, compared to the default choice $\widetilde{w}(1)=0$ choosing $\widetilde{w}(1)$ according to (4.14) typically reduces the deviations between the counters $C_{0}^{+}$and $C_{0}^{-}$from a factor on the order of one hundred to a factor of three or four ${ }^{\mathrm{b}}$.

\subsection{Temperature Reweighting}

So far, only the reweighting of simulation results in the chemical potential of the A particles has been discussed. Histogram reweighting straightforwardly extends to extrapolation in $\mu_{\mathrm{A}}$ and $\mu_{\mathrm{B}}$ by sampling a two dimensional histogram $P_{L}\left(\rho_{\mathrm{A}}, \rho_{\mathrm{B}} \mid z_{\mathrm{A}}, z_{\mathrm{B}}\right)$. But for the uniform sampling strategies just discussed, this greatly increases the computational effort. In SUS, the typical number of sub-intervals being sampled would increase from the order $10^{3}$ to the order of $10^{6}$. Also, effective ergodicity breaking (see section 2.2.1) may become a real problem when $N_{\mathrm{A}}$ and $N_{\mathrm{B}}$ are both constrained to small subintervals. In WL sampling, the number of MC steps required for a uniform sampling scales with the square of the number of weights (for a random walk) or worse (in the presence of hidden barriers such as the shape transition barrier discussed in chapter 5). Increasing the number of weights by a factor of $10^{3}$ hence results in an increase in CPU time by a factor of $10^{6}$, at least.

It turns out that a uniform sampling over the two dimensional OPD is not required. It suffices to use uniform sampling over $\rho_{\mathrm{A}}$ as described previously, and only to collect additional information about $N_{\mathrm{B}}$. To enable reweighting in $z_{\mathrm{B}}$, histograms $h_{N_{\mathrm{A}}}\left(N_{\mathrm{B}} \mid \mu_{\mathrm{B}}\right)$ are being sampled, which count the number of states with $N_{\mathrm{A}} \mathrm{A}$ particles and $N_{\mathrm{B}}$ $\mathrm{B}$ particles that were sampled in a simulation with a chemical potential $\mu_{\mathrm{B}}$ for the $\mathrm{B}$ particles. These histograms are to be understood as mutually independent one dimensional histograms in $N_{\mathrm{B}}$. They are not being directly compared to each other, and so $\mu_{\mathrm{A}}$ is irrelevant to them. These one dimensional histograms contain information about the microcanonical partition function, particularly

$$
h_{N_{\mathrm{A}}}\left(N_{\mathrm{B}} \mid \mu_{\mathrm{B}}\right) \approx \operatorname{const}\left(N_{\mathrm{A}}\right) \cdot \Omega\left(N_{\mathrm{A}}, N_{\mathrm{B}}\right) e^{\mu_{\mathrm{B}} N_{\mathrm{B}}},
$$

where the explicit $N_{\mathrm{A}}$-dependence of the normalization constant is meant as a remainder that the normalization constants between the histograms for different numbers of $\mathrm{A}$ particles usually differ.

\footnotetext{
${ }^{\mathrm{b}}$ Note that this factor increases the CPU time required but does not increase the statistical accuracy of $w(1)$, since that is dominated by the statistical accuracy of the counter with fewer entries.
} 
Relation 4.15 can be directly used to determine the change of the canonical partition functions (equation 2.7) under a change of the B fugacity, which is

$$
\begin{aligned}
f_{N_{\mathrm{A}}}\left(\bar{\mu}_{\mathrm{B}} \mid \mu_{\mathrm{B}}\right) & :=\frac{Z\left(N_{\mathrm{A}}, \bar{\mu}_{\mathrm{B}}\right)}{Z\left(N_{\mathrm{A}}, \mu_{\mathrm{B}}\right)} \stackrel{(2.7)}{=} \frac{\sum_{N_{\mathrm{B}}} e^{\bar{\mu}_{\mathrm{B}} N_{\mathrm{B}}} \Omega\left(N_{\mathrm{A}}, N_{\mathrm{B}}\right)}{\sum_{N_{\mathrm{B}}} e^{\mu_{\mathrm{B}} N_{\mathrm{B}} \Omega\left(N_{\mathrm{A}}, N_{\mathrm{B}}\right)}} \\
& \stackrel{(4.15)}{=} \frac{\sum_{N_{\mathrm{B}}=0}^{\infty} h_{N_{\mathrm{A}}}\left(N_{\mathrm{B}} \mid \mu_{\mathrm{B}}\right) e^{\left(\bar{\mu}_{\mathrm{B}}-\mu_{\mathrm{B}}\right) N_{\mathrm{B}}}}{\sum_{N_{\mathrm{B}}=0}^{\infty} h_{N_{\mathrm{A}}}\left(N_{\mathrm{B}} \mid \mu_{\mathrm{B}}\right)}
\end{aligned}
$$

Using the so-defined reweighting factors $f_{N_{\mathrm{A}}}\left(\bar{\mu}_{\mathrm{B}} \mid \mu_{\mathrm{B}}\right)$, the order parameter distribution can be extrapolated in both fugacities:

$$
\begin{aligned}
& P\left(N_{\mathrm{A}} \mid \bar{\mu}_{\mathrm{A}}, \bar{\mu}_{\mathrm{B}}\right) \stackrel{\stackrel{(4.1)}{=}}{=} Z\left(N_{\mathrm{A}}, \bar{\mu}_{\mathrm{B}}\right) \frac{e^{\bar{\mu}_{\mathrm{A}} N_{\mathrm{A}}}}{\mathcal{Z}\left(\bar{\mu}_{\mathrm{A}}, \bar{\mu}_{\mathrm{B}}\right)} \\
&= \underbrace{\frac{Z\left(N_{\mathrm{A}}, \bar{\mu}_{\mathrm{B}}\right)}{Z\left(N_{\mathrm{A}}, \mu_{\mathrm{B}}\right)}}_{\left(\stackrel{4.16)}{=} f_{N_{\mathrm{A}}}\left(\bar{\mu}_{\mathrm{B}} \mid \mu_{\mathrm{B}}\right)\right.} Z\left(N_{\mathrm{A}}, \mu_{\mathrm{B}}\right) \frac{e^{\left(\bar{\mu}_{\mathrm{A}}-\mu_{\mathrm{A}}\right) N_{\mathrm{A}}} e^{\mu_{\mathrm{A}} N_{\mathrm{A}}}}{\mathcal{Z}\left(\bar{\mu}_{\mathrm{A}}, \bar{\mu}_{\mathrm{B}}\right)} \\
& \stackrel{(4.1)}{=} f_{N_{\mathrm{A}}}\left(\bar{\mu}_{\mathrm{B}} \mid \mu_{\mathrm{B}}\right) e^{\left(\bar{\mu}_{\mathrm{A}}-\mu_{\mathrm{A}}\right) N_{\mathrm{A}}} P\left(N_{\mathrm{A}} \mid \mu_{\mathrm{A}}, \mu_{\mathrm{B}}\right) \frac{\mathcal{Z}\left(\mu_{\mathrm{A}}, \mu_{\mathrm{B}}\right)}{\mathcal{Z}\left(\bar{\mu}_{\mathrm{A}}, \bar{\mu}_{\mathrm{B}}\right)}
\end{aligned}
$$

As demonstrated by the curve labeled "full" in figure 4.2, performing uniform sampling over $N_{\mathrm{A}}$, collecting the histograms $h_{N_{\mathrm{A}}}$, and using (4.17) and (4.20) to reweight in both fugacities allows extrapolation over reasonable ranges $^{\mathrm{c}}$. From a single simulation at $z_{\mathrm{A}}=z_{\mathrm{B}}=1.72$, the $U_{1}$ cumulant on the locus $l_{\mathrm{MS}}$ (which is of course to be understood as only an example of an extrapolated observable) is very well approximated over the range $z_{\mathrm{B}} \approx[1.5 ; 2.1]$. The extrapolated data do, however, fail for $z_{\mathrm{B}}<1.5$, as clearly seen by the unexpected behavior. While such a failure of reweighting is easily identified by visual inspection of the curve, it becomes a problem when thousands of simulations need to be analyzed automatically. Therefore, a method to approximate $f_{N_{\mathrm{A}}}$ has been used that is more stable, and also reduces the need for disk space and CPU time.

\subsubsection{Reweighting from Reduced Histograms}

To reduce disk space it has been suggested to approximate the $h_{N_{\mathrm{A}}}$ as a Gaussian distribution that has the same average and variance as $h_{N_{\mathrm{A}}}{ }^{26}$. Since the reweighting of a Gaussian probability distribution can be performed analytically this reduces data storage for each histogram $h_{N_{\mathrm{A}}}$ to only two numbers, and also speeds up the evaluation of $f_{N_{\mathrm{A}}}$.

\footnotetext{
${ }^{\mathrm{c}}$ Reweight quality will of course depend on the system, the simulation fugacities, and the number of MC steps used. The results shown in figure 4.2 are to be understood as typical results for the pure WR model in two dimensions.
} 

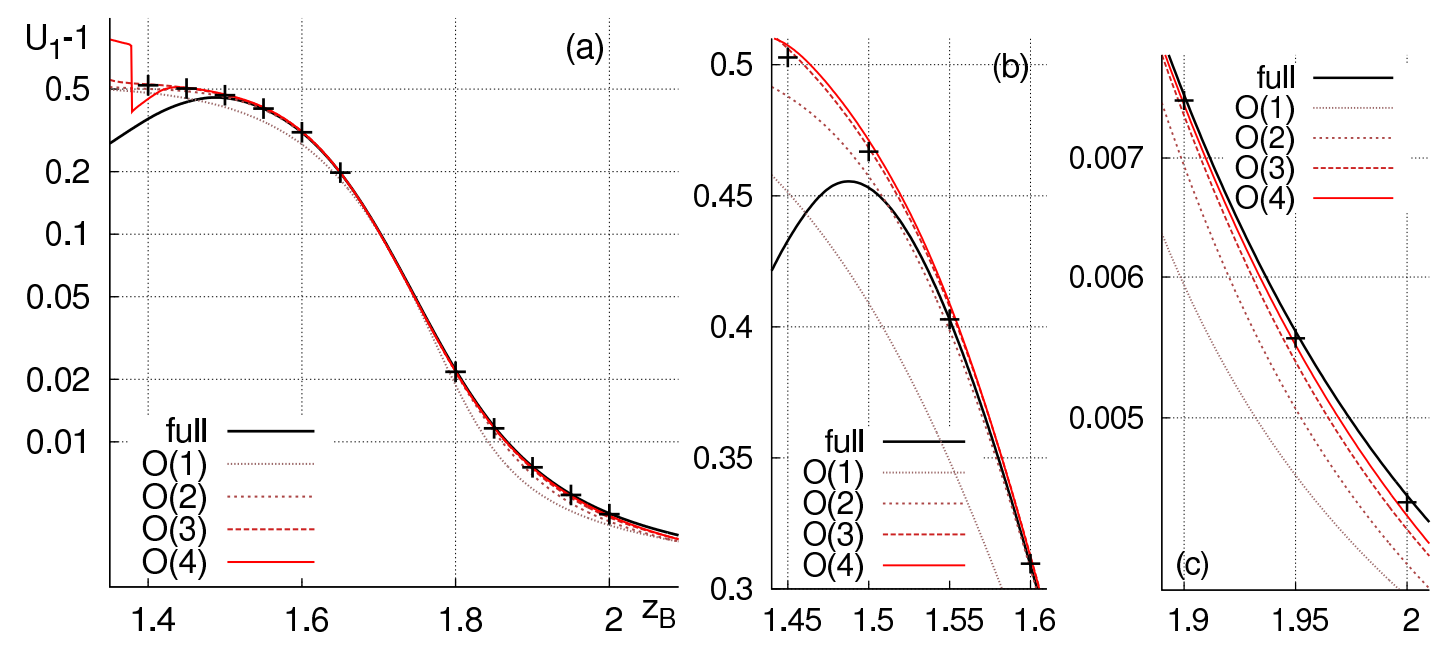

Figure 4.2: Quality of the histogram reweighting in $z_{\mathrm{B}}$ (equation 4.20) for a $2 \mathrm{D} L=20 \mathrm{WR}$ model on the example of the $U_{1}$ cumulant (all three images show $U_{1}-1$ as a function of $z_{\mathrm{B}}$, figures $\mathrm{b}$ and $\mathrm{c}$ are merely zooms into figure a). Different curves show results from the same successive umbrella sampling simulation performed at $z_{\mathrm{B}}=1.72$, but extrapolated with different methods; the locus $l_{\text {MS }}$ has been used in all cases. Black crosses are results from additional simulations that have not been reweighted. The curve labeled "full" corresponds to using full histograms $h_{N_{\mathrm{A}}}$ (equation 4.16). It yields the most accurate results, but at the cost of most CPU time, disk space, and a sudden failure of reweighting around $z_{\mathrm{B}}=1.5$. The curves $O(n)$ are calculated using the Taylor expanded approximations up to $n$ 'th order (see section 4.3.1). With increasing order they become more accurate but also more likely to break down, as seen in the fourth order at $z_{\mathrm{B}} \approx 1.4$. 
The Gaussian approximation works remarkably well and has been successfully employed before $^{24 ; 26}$. It turns out, however, that the assumption of a Gaussian is not required, and that the same result can be obtained by a Taylor expansion of $\log f_{N_{\mathrm{A}}}$ :

$$
\begin{aligned}
\log f_{N_{\mathrm{A}}}\left(\bar{\mu}_{\mathrm{B}} \mid \mu_{\mathrm{B}}\right) & =\underbrace{\log f_{N_{\mathrm{A}}}\left(\mu_{\mathrm{B}} \mid \mu_{\mathrm{B}}\right)}_{=0}+\left.\sum_{n=1}^{\infty} \frac{\left(\bar{\mu}_{\mathrm{B}}-\mu_{\mathrm{B}}\right)^{n}}{n !} \frac{\partial^{n} \log f_{N_{\mathrm{A}}}\left(\bar{\mu}_{\mathrm{B}} \mid \mu_{\mathrm{B}}\right)}{\partial \bar{\mu}_{\mathrm{B}}^{n}}\right|_{\bar{\mu}_{\mathrm{B}}=\mu_{\mathrm{B}}} \\
& =\left.\sum_{n=1}^{\infty} \frac{\left(\bar{\mu}_{\mathrm{B}}-\mu_{\mathrm{B}}\right)^{n}}{n !} \frac{\partial^{n} \log Z\left(N_{\mathrm{A}}, \bar{\mu}_{\mathrm{B}}\right)}{\partial \bar{\mu}_{\mathrm{B}}^{n}}\right|_{\bar{\mu}_{\mathrm{B}}=\mu_{\mathrm{B}}}
\end{aligned}
$$

The derivatives of a partition function's logarithm can be expressed via the first moments of the corresponding probability distribution (see appendix A). In this case the probability distribution is the normalized histogram $h_{N_{\mathrm{A}}}\left(N_{\mathrm{B}} \mid \mu_{\mathrm{B}}\right)$, and so for performing an expansion up to $n$ 'th order requires only the first $n$ moments of $h_{N_{\mathrm{A}}}$. For example, the expansion to second order is

$$
\begin{aligned}
& \log f_{N_{\mathrm{A}}}\left(\bar{\mu}_{\mathrm{B}} \mid \mu_{\mathrm{B}}\right)=\underbrace{\left.\frac{\partial \log Z\left(N_{\mathrm{A}}, \bar{\mu}_{\mathrm{B}}\right)}{\partial \bar{\mu}_{\mathrm{B}}}\right|_{\bar{\mu}_{\mathrm{B}}=\mu_{\mathrm{B}}}}_{\stackrel{(A .7)}{=}\left\langle N_{\mathrm{B}}\right\rangle}\left(\bar{\mu}_{\mathrm{B}}-\mu_{\mathrm{B}}\right) \\
& +\underbrace{\left.\frac{\partial \log Z\left(N_{\mathrm{A}}, \bar{\mu}_{\mathrm{B}}\right)}{\partial \bar{\mu}_{\mathrm{B}}}\right|_{\bar{\mu}_{\mathrm{B}}=\mu_{\mathrm{B}}}}_{\stackrel{\left(A_{.8}\right)}{=}\left\langle N_{\mathrm{B}}{ }^{2}\right\rangle-\left\langle N_{\mathrm{B}}\right\rangle^{2}} \frac{\left(\bar{\mu}_{\mathrm{B}}-\mu_{\mathrm{B}}\right)^{2}}{2} \\
& \Rightarrow f_{N_{\mathrm{A}}}\left(\bar{\mu}_{\mathrm{B}} \mid \mu_{\mathrm{B}}\right)=\exp \left[\left\langle N_{\mathrm{B}}\right\rangle\left(\bar{\mu}_{\mathrm{B}}-\mu_{\mathrm{B}}\right)+\left(\left\langle N_{\mathrm{B}}^{2}\right\rangle-\left\langle N_{\mathrm{B}}\right\rangle^{2}\right) \frac{\left(\bar{\mu}_{\mathrm{B}}-\mu_{\mathrm{B}}\right)^{2}}{2}\right] \text {. }
\end{aligned}
$$

This is precisely the result one would obtain from assuming that the $h_{N_{\mathrm{A}}}$ have Gaussian shape $^{\mathrm{d}}$. Hence, the Gaussian approximation is not required, and should be replaced with the more general Taylor expansion of $\log f_{N_{\mathrm{A}}}$, which also straightforwardly extends to the reweighting in multiple parameters.

The results of reweighting according to an expansion up to fourth order are shown in figure 4.2, labeled $O(1), \ldots O(4)$. As seen, already low orders of expansion approximate the cumulant curve well, and are stable over a larger range. The slightly worse accuracy of the extrapolated data is unproblematic, as very high precision is only required in a small fugacity range (usually around $z_{\mathrm{B}, \mathrm{cr}}$ ). This can be achieved by simply simulating in this region after having identified it with extrapolated trial simulations. Tests on a Taylor expansion of $f_{N_{\mathrm{A}}}$ (rather than $\log f_{N_{\mathrm{A}}}$ ), which may appear to be an alternative method, have shown an extremely bad convergence behavior: A second order expansion of $\log f_{N_{\mathrm{A}}}$ has been found to be more accurate than an expansion of $f_{N_{\mathrm{A}}}$ up to twentieth order.

\footnotetext{
${ }^{\mathrm{d}}$ It is a property of Gaussian distributions that higher order derivatives in the expansion vanish ${ }^{72}$.
} 


\subsubsection{Multiple Histogram Methods}

In the pure model, the $z_{\mathrm{B}}$-range that data from a single simulation can be extrapolated to is sufficiently large to capture the interesting region (as demonstrated in figure 4.2). In some cases, however, the use of multiple simulation points can be required to estimate an observable over a large range of fugacities. The combination of many simulations in the optimal way is known as the multiple histogram method ${ }^{53 ; 73}$ or weighted histogram analysis method $^{74}$. For the work presented here ${ }^{\mathrm{e}}$ these methods are not directly applicable, as only the first few moments of the $h_{N_{\mathrm{A}}}$ and not the complete histograms are being stored. Thus, a method in the spirit of finding the best combined estimate from all simulations has been developed that is compatible with the Taylor expansion introduced in section 4.3.1.

As knowing the order parameter distribution is equivalent to knowing a set of best weights $w_{n}$ (equation 4.9), knowing the OPD is also equivalent to knowing all weight differences $\Delta_{n}:=w_{n+1}-w_{n}$. A set of $i=1,2, \ldots, M$ independent successive umbrella sampling simulations each performed at different fugacities provides $M$ estimates $\Delta_{n}^{(i)}$ for this value. These are

$$
\Delta_{n}^{(i)}:=w_{n+1}^{(i)}-w_{n}^{(i)}+\left(\mu_{\mathrm{A}}-\mu_{\mathrm{A}}^{(i)}\right)+\log f_{n+1}^{(i)}\left(\mu_{\mathrm{B}} \mid \mu_{\mathrm{B}}^{(i)}\right)-\log f_{n}^{(i)}\left(\mu_{\mathrm{B}} \mid \mu_{\mathrm{B}}^{(i)}\right),
$$

where the upper indices $(i)$ indicate that the respective values are obtained from simulation $i$ at chemical potentials $\mu_{\mathrm{A}}^{(i)}$ and $\mu_{\mathrm{B}}^{(i)}$. If to each of these values a statistical variance $v_{n}^{(i)}$ can be associated, then the best combined estimates for the weight differences become a weighted average:

$$
\Delta_{n}=\frac{1}{\sum 1 / v_{n}^{(i)}} \sum \frac{\Delta_{n}^{(i)}}{v_{n}^{(i)}} .
$$

The problem of finding a proper analogue of multiple histogram reweighting is therefore related to the problem of finding proper estimates for the variances $v_{n}^{(i)}$.

Deriving this variance from first principles is far from trivial because of correlations between the MC moves used to sample the SUS subintervals, and because of remanence effects such as the shape transitions discussed in chapter 5 . Thus, the $v_{n}^{(i)}$ have been approximated as the sum of the variance of the original difference in the weights and a variance associated to the reweighting in $z_{\mathrm{B}}$ (there is no statistical error associated to the reweighting in $z_{\mathrm{A}}$ ). The statistical variance of the weight difference at the original fugacity is only due to uncertainties in the counters $C_{n}^{(i)+}$ and $C_{n}^{(i)-}$ (defined in section 4.2.2) which are assumed as independent Poissonian variables. In this case, the variance can be

\footnotetext{
${ }^{\mathrm{e}} \mathrm{I}$ used the multiple histogram method in the form described in the book of Barkema ${ }^{53}$ for an analysis of the $2 \mathrm{D}$ Zwanzig model that is mentioned in section 2.1.4 but not discussed in detail ${ }^{49}$.
} 
approximated as

$$
\begin{aligned}
\operatorname{var}\left[w_{n+1}^{(i)}-w_{n}^{(i)}\right] & =\operatorname{var}\left[\log C_{n}^{(i)+}-\log C_{n}^{(i)-}\right] \\
& =\frac{1}{C_{n}^{(i)+}}+\frac{1}{C_{n}^{(i)-}}
\end{aligned}
$$

Assuming both counters to be uncorrelated Poissonian variables obviously underestimates the error estimate. But as long as the variances for all simulations are underestimated similarly, this can still result in an acceptable best estimate.

In a second order Taylor expansion of $f_{N_{\mathrm{A}}}$ (equation 4.22) the terms contributing to the statistical variance of the reweighting contribution are the average particle number $\left\langle N_{\mathrm{B}}\right\rangle$ and the variance $\left\langle\left(N_{\mathrm{B}}-\left\langle N_{\mathrm{B}}\right\rangle\right)^{2}\right\rangle$ of the histogram $h_{N_{\mathrm{A}}}$ that has been sampled. To estimate a variance for the reweighting contribution, these two terms are assumed to be independent random variables. Furthermore, all the entries in the histograms are assumed to be independent random Poissonian variables (with the same reasoning as in case of the counters of the SUS subinterval). Consider such a histogram that has been sampled with $C$ entries in total. The variance of the average of some function $g\left(N_{\mathrm{B}}\right)$ is

$$
\begin{aligned}
\operatorname{var}[\langle g\rangle] & =\operatorname{var}\left[\sum_{N_{\mathrm{B}}} g\left(N_{\mathrm{B}}\right) \frac{h_{N_{\mathrm{A}}}\left(N_{\mathrm{B}}\right)}{C}\right]=\sum_{N_{\mathrm{B}}} \operatorname{var}\left[g\left(N_{\mathrm{B}}\right) \frac{h_{N_{\mathrm{A}}}\left(N_{\mathrm{B}}\right)}{C}\right] \\
& =\sum_{N_{\mathrm{B}}} \frac{g\left(N_{\mathrm{B}}\right)^{2}}{C^{2}} \underbrace{\operatorname{var}\left[h_{N_{\mathrm{A}}}\left(N_{\mathrm{B}}\right)\right]}_{=h_{N_{\mathrm{A}}}\left(N_{\mathrm{B}}\right)}=\frac{1}{N} \sum_{N_{\mathrm{B}}} g\left(N_{\mathrm{B}}\right)^{2} \frac{h_{N_{\mathrm{A}}}\left(N_{\mathrm{B}}\right)}{C} \\
& =\frac{1}{N}\left\langle g^{2}\right\rangle .
\end{aligned}
$$

The variances of the reweighting contributions in equation 4.23 are therefore approximated as

$$
\begin{array}{ll}
\stackrel{(4.22)}{=} & \operatorname{var}\left[\log f_{N_{\mathrm{A}}}\left(\bar{\mu}_{\mathrm{A}} \mid \bar{\mu}\right)\right] \\
\stackrel{(4.27)}{=} & \overline{1}\left[\left\langle N_{\mathrm{B}}\right\rangle\right]\left(\bar{\mu}_{\mathrm{B}}-\mu_{\mathrm{B}}\right)^{2}+\operatorname{var}\left[\left\langle\left(N_{\mathrm{B}}-\left\langle N_{\mathrm{B}}{ }^{2}\right\rangle\right)^{2}\right\rangle\right] \frac{\left(\bar{\mu}_{\mathrm{B}}-\mu_{\mathrm{B}}\right)^{4}}{4} \\
& \left.\left.C \mu_{\mathrm{B}}\right)^{2}+\left\langle\left(N_{\mathrm{B}}-\left\langle N_{\mathrm{B}}\right\rangle\right)^{4}\right\rangle \frac{\left(\bar{\mu}_{\mathrm{B}}-\mu_{\mathrm{B}}\right)^{4}}{4}\right],
\end{array}
$$

where averages $\langle\cdot\rangle$ refer to averages over $h_{N_{\mathrm{A}}}$. The index for the simulation number has been dropped for better readability. The term $\left\langle\left(N_{\mathrm{B}}-\left\langle N_{\mathrm{B}}\right\rangle\right)^{4}\right\rangle$ can be evaluated in terms of the first four moments of $h_{N_{\mathrm{A}}}$, so it does not require full histograms, but can also be evaluated by knowing only its first moments.

Using equations (4.28) and (4.26) the variance $\Delta_{n}^{(i)}$ in (4.23) and therefore the best estimate for the local weight difference for a set of best weights in (4.24) can be estimated. The construction of the best estimate order parameter distribution is then straightforward 


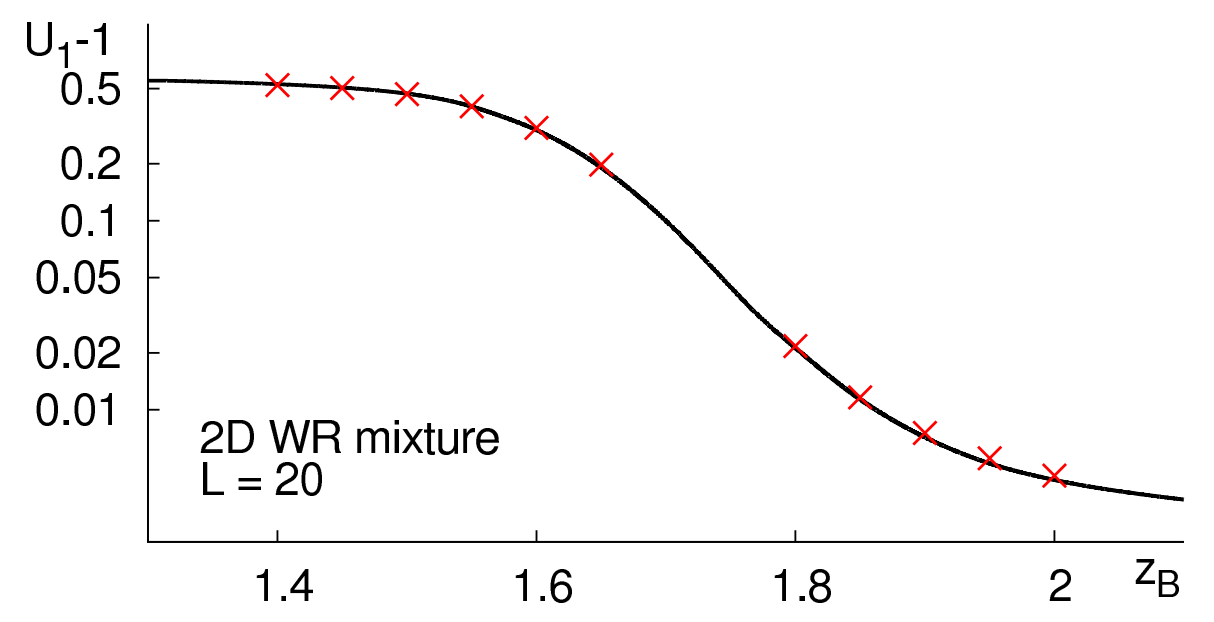

Figure 4.3: Cumulant values $U_{1}$ obtained from the multiple histogram method introduced in the text. The black curve is the best combined estimate from simulations performed at $z_{\mathrm{B}}=1.45,1.65,1.85,2.05,2.25$, and agrees well with the red crosses, which are results from direct non-reweighted simulations at the respective fugacities.

(cf. equation 4.9). A result of using this multiple histogram method is shown in figure 4.3, which demonstrates that despite the very dubious assumption of independent Poissonian entries in the histograms good accuracy and stability over a large range is achieved.

\subsection{Summary}

In this section, the histogram method has been introduced, which is required for the analysis of Widom-Rowlinson models by means of Monte Carlo simulations. It has been demonstrated that for a reliable histogram reweighting the computer simulation should not sample states according to the Boltzmann distribution. Instead, the simulation must be modified such that an approximately equal statistical accuracy over an interval $\rho_{\mathrm{A}} \in\left[0, \rho_{\mathrm{A}, \max }\right]$ is achieved. Two commonly-used method that achieve this, Wang-Landau sampling and successive umbrella sampling, have been described.

An approximation for the reweighting in $z_{\mathrm{B}}$ via Taylor expansions has been introduced that, in contrast to normal histogram reweighting, does not require to store and evaluate full histograms, but only requires their first few moments. This method reduces the amount of disk space and CPU time required by approximately a factor of $25^{\mathrm{f}}$ and straightforwardly extends to higher-dimensional histograms. An improved stability of this method for reweighting over large distances (at the expense of some accuracy for reweighting over intermediate distances) has been demonstrated. Because of this property

\footnotetext{
${ }^{\mathrm{f}}$ Measured on the calculation of the $U_{1}$ cumulant for a $2 \mathrm{D}$ pure WR model of size $20 \times 20$ and a Taylor expansion to 4 th order.
} 
and the high level of automatization required in analyses of the Widom-Rowlinson model in the presence of quenched disorder, the reweighting via Taylor expansion (to second order) has been chosen as the default method for reweighting in $z_{\mathrm{B}}$. Finally, a multiple histogram method based on this approximation has been developed. This multiple histogram method further extends the extrapolation range, which is required for the simulation of the two dimensional Widom-Rowlinson mixture in the presence of random obstacles with an unequal interaction to the A and B particles (chapter 8). 



\title{
Phase Coexistence and Sampling Barriers
}

\subsection{Phase Coexistence}

\begin{abstract}
Above the critical fugacity a WR fluid can exist in two thermodynamic stable states, the low density gas and the high density liquid phase. But since the uniform sampling strategies introduced in the previous chapter also yield information about the region between the liquid and the gas peak in the order parameter distribution the state of the system at these intermediate densities is also of interest - and can in fact provide valuable information. At these intermediate densities, the system is not in a uniform state, but a state in which the liquid and the gas phase $\mathrm{e}^{\mathrm{a}}$ exist simultaneously, both occupying a part of the total system volume and separated by an interface. The phase occupying less volume is the minority phase; its volume shall be denoted by $V_{\mathrm{M}}$. Depending on the system's dimension, its shape, and the ratio of the minority volume to the total volume $V_{\mathrm{M}} / V$, the arrangement of the two phases can assume different shapes.
\end{abstract}

In two-dimensional systems simulated on squares with periodic boundary conditions (topologically such systems are tori) the two possible arrangements for the phases are the ones shown in figure 5.1: a minority phase droplet (left image) and a slab geometry (right image), in which both phases arrange in system-spanning slabs parallel to the shortest system sides. The possible configurations in a three dimensional system are shown in 5.2. They are the droplet and the slab geometry known from the two-dimensional system, and an additional stalk geometry that has the form of a cylinder spanning the system parallel to its shortest axis ${ }^{75}$.

The appearance of these shapes can be understood as stemming from an interface contribution to the free energy (note that the dominating bulk contributions are already fixed by $z_{\mathrm{A}}, z_{\mathrm{B}}$ and $V_{\mathrm{M}}$ but do not determine the shapes). This contribution equals the

\footnotetext{
an the previous chapters, the term "pseudophase" has been used to emphasize that the states seen in a simulation are finite-size states and only become proper phases in the thermodynamic sense in the thermodynamic limit. Since in the pure WR model it is established that proper phases develop in the thermodynamic limit, this distinction will be dropped in this chapter for readability.
} 

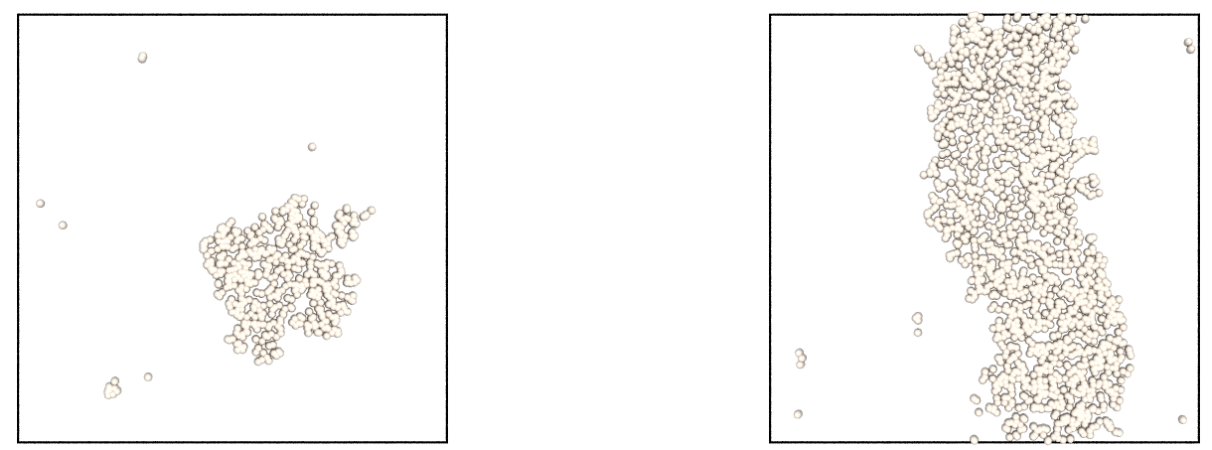

Figure 5.1: The two possible shapes that the minority phase can assume in a two-dimensional system are the droplet shape shown in the left and the slab shape shown in the right image.
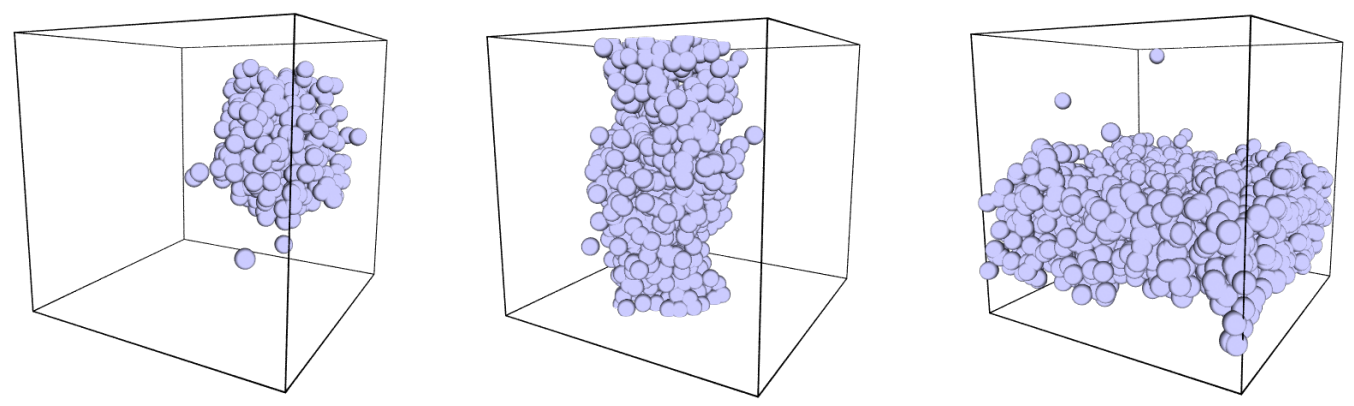

Figure 5.2: Simulation snapshots of the possible distinct shapes that the minority phase can assume in a 3D WR mixture at phase coexistence. With increasing volume of the minority phase these are, from left to right, a spherical droplet, a cylinder spanning the system (the stalk), and a cuboid spanning the system in two dimensions parallel to the boundaries (the slab geometry).
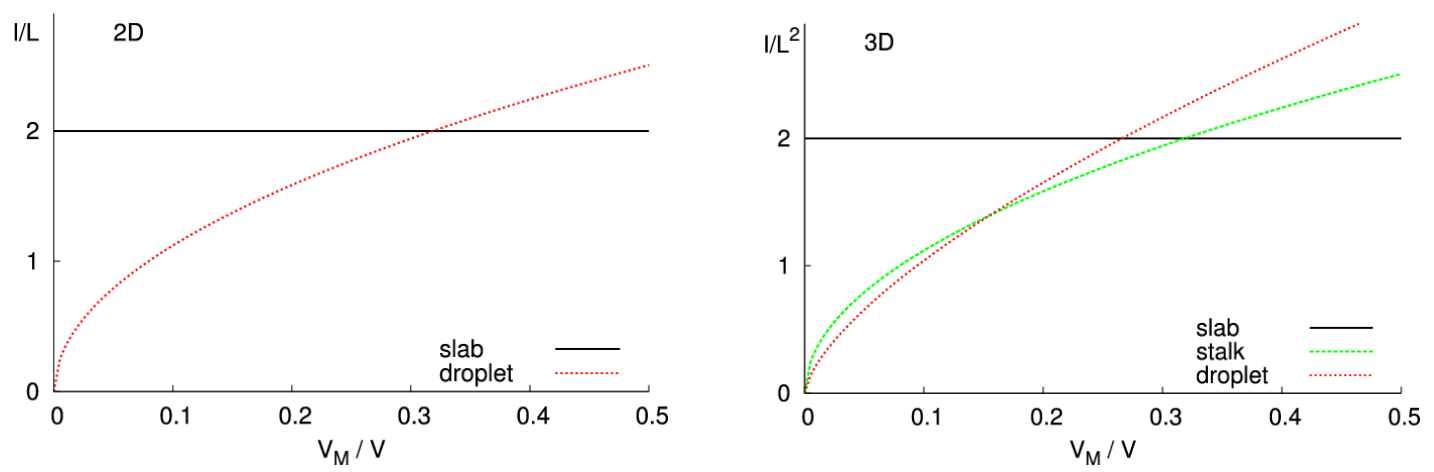

Figure 5.3: Interface sizes of the different possible minority phase shapes for cubic systems with side-length $L$ and periodic boundary conditions in two (left plot) and three dimensions (right plot). The functions for the amount of interface assume perfect geometrical shapes (sphere, cylinder, axes-parallel cuboid). In both cases the favored shape is a function of the minority phase volume $V_{\mathrm{M}}$. 

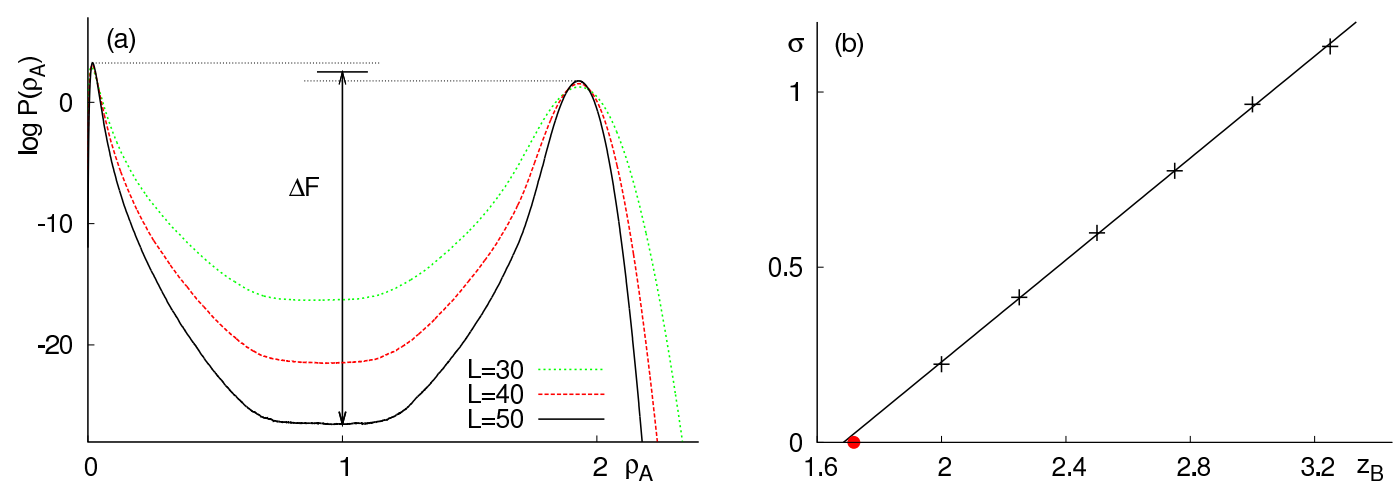

Figure 5.4: From OPDs, a free energy barrier $\Delta F$ associated to the formation of an interface between coexisting phases can be extracted. Figure (a) shows OPDs for the 2D WR system at $z_{\mathrm{B}}>z_{\mathrm{B}, \mathrm{cr}}$ for different system sizes. $\Delta F$ is defined as the difference between the average logarithms of the peaks and the logarithm of the in-between minimum. In this case, the flat region between the peaks indicates a slab geometry, which allows to extract the interface tension $\sigma$ via equation 5.2. The interface tension vanishes at the critical point. As shown in figure (b), the measurement and interpolation of interface tensions can be used to estimate the critical fugacity (marked by the red dot).

amount of interface ${ }^{\mathrm{b}}$ between the coexisting phases times the interface tension $\gamma^{77}$. To minimize the free energy, the phases arrange such that the interface between them is minimized which, depending on $V_{\mathrm{M}}$, can result in either of the shapes shown in figure 5.1 and 5.2. The amount of interface $I$ caused by the different shapes as a function of the minority phase volume is shown in figure 5.3. As clearly seen, different coexistence shapes are assumed for different minority phase volumes.

\subsubsection{The Slab Geometry: Extracting the Interface Tension}

Of particular interest is the slab geometry, which in two and three dimensional systems always is assumed for $V_{\mathrm{M}} / V \approx 0.5$. If the system is sufficiently large, the two interfaces in the slab geometry do not interact with each other, and the free energy of such a system equals that of either of the two coexisting phases plus the contribution from the interface. Since in the slab geometry $I$ does not depend on $\rho_{\mathrm{A}}$, the logarithm of the OPD (which is directly related to the free energy - see equation 4.1) is flat there. Conversely, since for all other coexistence shapes the amount of interface varies with $V_{\mathrm{M}}$ the densities for which the slab geometry is assumed can be inferred from the OPD by identifying its flat regions (see figure 5.4).

\footnotetext{
${ }^{\mathrm{b}}$ The amount of interface generally depends on the length scale that it is measured on. The usage of the term here refers to a macroscopic scale ${ }^{76}$.
} 


\section{The Free Energy Barrier}

On the locus of phase equilibrium, the free energy barrier $\Delta F$, which is defined as the difference between the average logarithm of the liquid and the gas peak minus the logarithm of the in-between minimum, is due to an interface contribution

$$
\Delta F=\sigma I,
$$

where $\sigma$ is the interface tension and $I$ is the amount of interface between the two coexisting phases. In the slab geometry, the interface is known to be $I=2 L^{d-1}$. Thus $\sigma$ can be extracted from an OPD via the relation

$$
\Delta F=2 \sigma L^{d-1},
$$

where it now is assumed that the minimum between the two peaks in the OPD corresponds to a slab geometry, indicated by a flat region in the OPD ${ }^{c}$. As shown in figure 5.4(b), extracting the interface tensions can in principle be used to approximate the critical point, since $\sigma$ vanishes at $z_{\mathrm{B}, \mathrm{cr}}$.

\subsubsection{Shape Transition}

For the Monte Carlo simulation described in the previous chapters, the transitions between the different possible coexistence shapes pose a problem. Consider a two-dimensional system of size $L \times L$ in a droplet state that crosses the droplet $\rightarrow$ slab transition at $V_{\mathrm{M}} / V=1 / \pi$. The correct equilibrium state is the slab, but the radius of the droplet is only $2 L / \pi$, which is smaller than the diameter of the lateral extension $L$ that the slab spans. Monte Carlo simulations performing only single particle moves can only transit from the droplet to the slab geometry by the construction of intermediate states, in this case lens-shaped minority volumes ${ }^{78}$. However, in these intermediate shapes the interface between the coexisting phases is larger than in the equilibrium shapes by some excess interface $\Delta I$, causing an ensemble of such intermediate states to have an excess free energy $\gamma \Delta I$ compared to an ensemble of equilibrium states. Since the excess interface grows with the lateral extension $L$ of the system, the probability for the appearance of the intermediate states during a Monte Carlo simulation using single insertion and removal steps decreases exponentially ${ }^{78}$.

A similar reasoning holds true for the shape transition in the 3D system also, since neither the radius of the droplet at the droplet $\leftrightarrow$ stalk transition nor the radius of the stalk at the stalk $\leftrightarrow$ slab transition equal the lateral extension of the system. Again, an increased suppression of the appearance of the intermediate states with increasing system size is expected.

\footnotetext{
${ }^{\mathrm{c}}$ In practice, the $d=3$ systems allow to extract reasonable values for $\sigma$ even for system sizes that are too small to show a flat region in the OPD. Seeing a plateau is therefore not strictly required.
} 

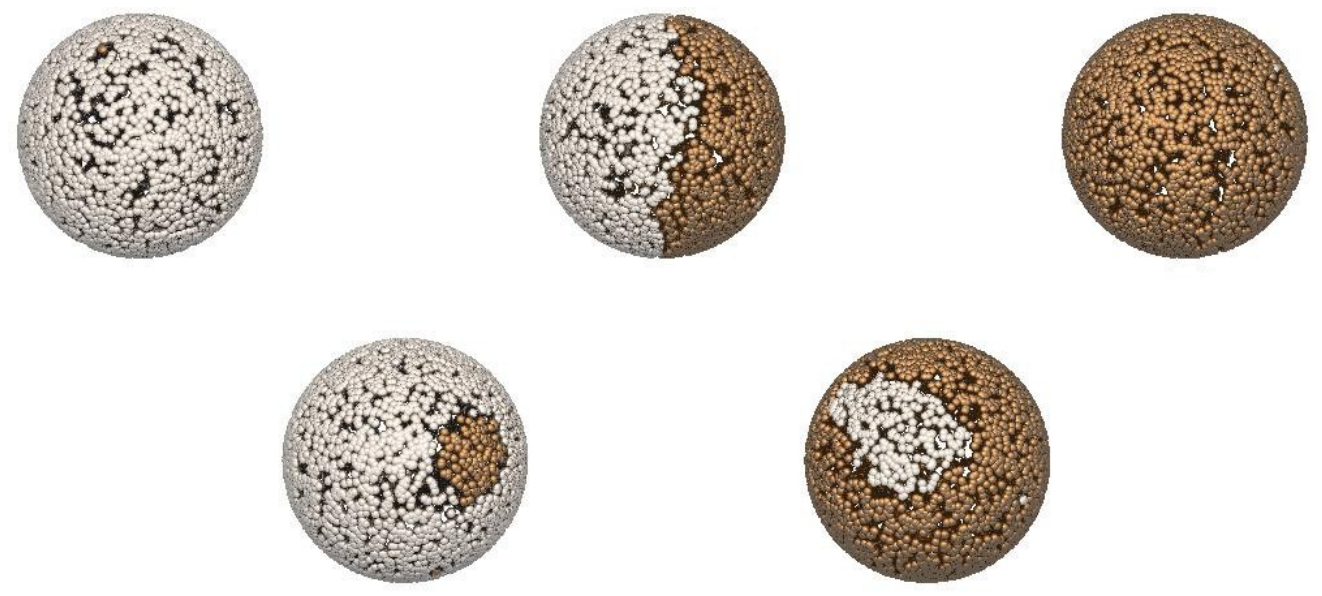

Figure 5.5: In simulations of the two-dimensional WR model on the surface of a sphere, no shape transitions occur. Topologically, the only coexistence shape is that of two droplets. The increase of one droplet's volume at the expense of the other's is always continuous and does not require single insertion/removal steps to create unfavorable intermediate states.

As a direct result of this reduced probability to construct intermediate states, the probability for a Monte Carlo simulation with single particle moves to perform the shape transitions at the correct transition points also decreases exponentially. These shape transition barriers limit the size of systems that can be properly simulated at phase coexistence. In principle, one could imagine defining an order parameter for the shape formed by the minority phase and using a uniform sampling scheme to smoothly guide the simulation through the shape transition. But this not only adds an additional layer of complexity to the simulation; it is also not clear what a suitable order parameter would look like. In any case, it is probably computationally expensive (compared to the particle number), making its use unattractive.

\subsection{Simulations on Hyperspheres}

An alternative way to circumvent the problem with the shape transitions has (for a twodimensional system) already been proposed in $1990^{79}$, namely to perform the simulations of a $d$-dimensional system on the surface of a $d+1$-dimensional sphere rather than a $d$-dimensional box with periodic boundary conditions. There, the slab geometry, which is caused by the system abusing the periodic boundary conditions, is absent. The only shape that the minority phase assumes is that of a continuously growing droplet (see figure 5.5). According to the discussion in the previous section, the absence of a shape transition should improve the efficiency of simulations sampling through the coexistence 
region, at least in the limit of large distance from the critical point and large systems.

In a related scenario, a 2D Ising model being simulated on the surface of a $3 \mathrm{D}$ cube, such an efficiency improvement has been noted ${ }^{78}$ in multicanonical simulations ${ }^{64 ; 80}$. This analysis is closely related to the simulation of an off-lattice system of a sphere, but differs in two aspects: First, the transition between droplet and strip is not entirely eliminated but traded against other, albeit less severe, shape transitions ${ }^{78}$. Second, the spins on the edges of the grid only have three nearest neighbors, not four. Thus, the system on the surface of the cube contains "impurities", and strictly speaking is not completely equivalent to the standard system geometry, even in the thermodynamic limit.

The remainder of this chapter introduces simulations of the WR model on a true hypersphere, discusses the effect on simulation performance, and demonstrates that the performance increase that is seen indeed owes to the absence of shape transitions.

\subsubsection{Implementation Details for the WR Model}

For the simulation of a $d$-dimensional system with spherical geometry the surface of a sphere embedded in a $(d+1)$-dimensional Euclidean space is used. The surface of the $(d+1)$-dimensional sphere is chosen as equal to the $d$-dimensional volume of the system that shall be simulated. This fixes the radius $R$ of the $(d+1)$-dimensional sphere. Particle positions on the sphere are described by $(d+1)$-dimensional Euclidean vectors, where the location $\mathbf{r}_{i}$ of each particle $i$ has to satisfy the "on sphere" condition $\mathbf{r}_{i} \cdot \mathbf{r}_{i}=R^{2}$. The Monte Carlo simulation of the system on the spherical geometry is identical to the simulation on the torus except for the construction of uniformly distributed random locations for particles and the measurement of distances.

Uniformly distributed random locations are obtained by independently choosing the $d+1$ coordinates of a vector $\mathbf{r}_{i}$ from a uniform distribution over $[-R ; R]$. For each position, this is repeated until the coordinates satisfy $\mathbf{r}_{i} \cdot \mathbf{r}_{i} \leq R^{2}$, in which case the vector is rescaled to $\mathbf{r}_{i} \cdot \mathbf{r}_{i}=R^{2}$, and accepted as the new random location.

On a surface of a sphere the shortest distance between any two points $\mathbf{r}_{i}$ and $\mathbf{r}_{j}$ lies on a great circle. The on-sphere distance $d$ between these two points is thus given by

$$
d=R \cos ^{-1}\left(\frac{\mathbf{r}_{i} \cdot \mathbf{r}_{j}}{R^{2}}\right)
$$

To test the intersection of two particles one only needs to check whether $d$ is smaller than some threshold value $a$, and does not need the actual value of $d$. This allows to eliminate the computation of a trigonometric function for this check, since

$$
d<a \stackrel{(5.3)}{\Leftrightarrow} \mathbf{r}_{i} \cdot \mathbf{r}_{j}>R^{2} \cos (a / R),
$$

where the term $R^{2} \cos (a / R)$ is a constant over the course of the simulation. 

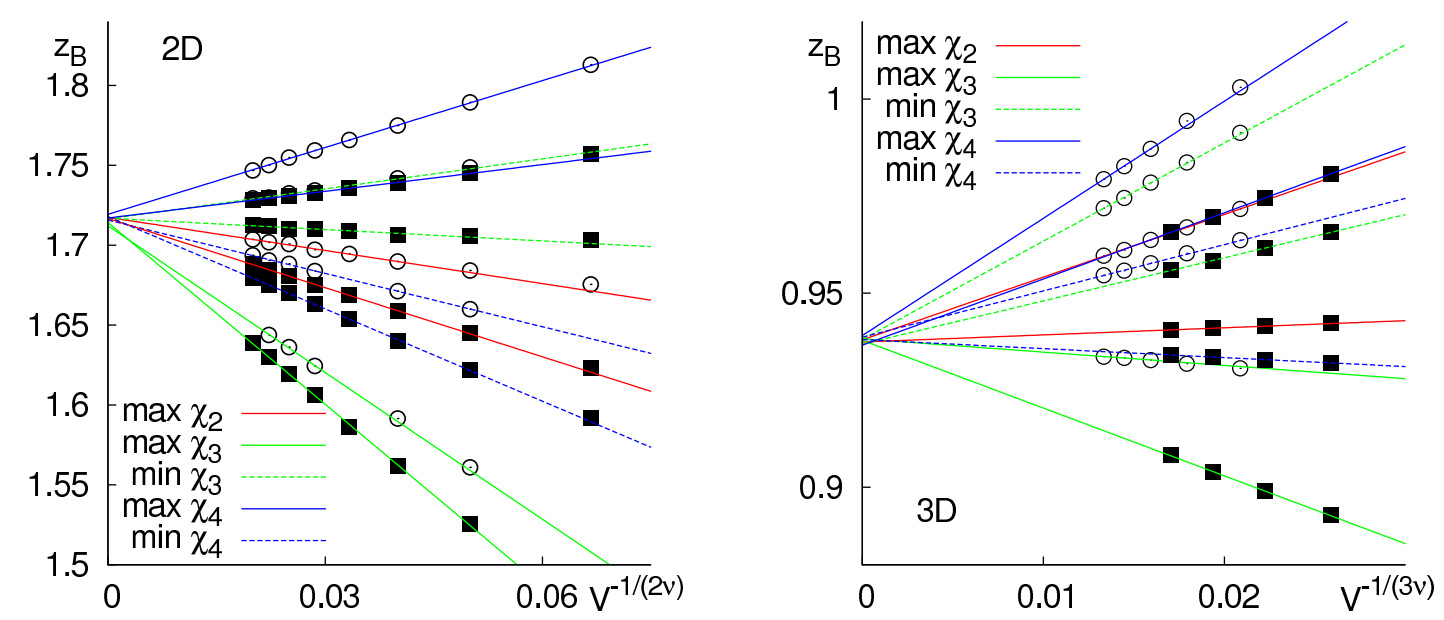

Figure 5.6: Finite size scaling of the extrema in $\chi_{2}, \chi_{3}$, and $\chi_{4}$ for simulation results from spherical (hollow circles) and toroidal (filled squares) systems. Left plot is for the two- and the right for the three-dimensional Widom Rowlinson model. For finite system sizes there is a distinctive difference in the peak locations between the two geometries, but both geometries predict the same critical fugacity in the thermodynamic limit.

\subsubsection{Transcription Rules: How to chose $a$ ?}

There is no unique way to transfer a WR model of a given size to a sphere, and there are three possible choices for the interaction distance $a$. The simplest choice is the equaldistance mapping, where $a=1$. The other two are the equal-particle-volume mapping, where on-sphere particle radii are chosen such that the integrated particle volume equals that of the particles on the toroidal geometry, and the equal-excluded-volume mapping where said equality holds for the volume that an A particle excludes for B particles. These three choices lead to slightly different results for the finite systems, but in the thermodynamic limit where the curvature integrated over the volume of a particle (or the exclusion volume) vanishes, they become equal. All results shown here use the equal-distance mapping.

\subsubsection{Locating the Critical Point}

The analysis of the order parameter distributions obtained from simulations on a hypersphere is identical to that of OPDs obtained from simulations in a toroidal system. For the length-scale $L$ that appears in finite-size scaling $L=V^{1 / d}$ has been chosen. In figure 5.6 the finite-size scaling of the pseudo-critical points' locations are shown for spherical and toroidal WR systems in two and three dimensions. As expected, the points of pseudocriticality differ between the two geometries, but in both cases the same thermodynamic limit is found. 


\begin{tabular}{l|cc} 
& Setup 1 & Setup 2 \\
\hline \hline Dimension & 2 & 3 \\
Fugacity $\left(z_{\mathrm{A}}=z_{\mathrm{B}}\right)$ & 2.5 & 1.5 \\
Runs & 40 & 20 \\
Tuning locus & $l_{\mathrm{MS}}$ & $l_{\mathrm{MS}}$ \\
\hline Wang-Landau Sampling & & \\
$\delta$ (first iteration) & $\log (1.2)$ & $\log (1.6)$ \\
$\delta$ adjustment & $\delta \rightarrow \delta / 2$ & $\delta \rightarrow \delta / 2$ \\
iterations & 20 & 21 \\
\hline Successive Umbrella Sampling & & \\
Window Size & 2 & - \\
MC Steps per Window & $5 \cdot 10^{7}$ & - \\
\hline
\end{tabular}

Table 5.1: The simulation parameters used for the performance analyses shown in this chapter. The number of runs is the number of independent simulations performed for each uniform sampling method and each geometry. The number of MC steps per window in successive umbrella sampling counts all attempted MC steps, including those that were rejected.

\subsubsection{Autocorrelation Times}

On the example of the two-dimensional case it is now tested if exponential slowing down is indeed less pronounced on a sphere. Consider a simulation using perfect weights in the sense of equation 4.9 sampling the interval $N_{\mathrm{A}} \in\left[0 ; N_{\mathrm{A}}^{\max }\right]$ using the single particle moves introduced in section 4.2. Because of the perfect (or near-perfect) weights being used, the simulation samples the interval uniformly, even without employing Wang-Landau sampling or successive umbrella sampling. If there are no hidden barriers in the system, then the simulation performs a random walk on the interval $\left[0 ; N_{\mathrm{A}}^{\max }\right]$. The autocorrelation time $\tau$, defined as the average number of MC steps the simulation takes to randomly wander from a state with $N_{\mathrm{A}}=0$ to a state with $N_{\mathrm{A}}=N_{\mathrm{A}}^{\max }$ and back to a state with $N_{\mathrm{A}}=0$, then scales with the system volume as proportional to $V^{2}$.

In the presence of shape transitions, the autocorrelation time instead shows an exponential increase with the system size, stemming from the exponentially dropping probability to transfer between the shapes. This is shown in figure 5.7(a), where the autocorrelation time for simulations on toroidal and on spherical WR systems is shown as a function of volume. The weights used in the simulations were obtained from previous high-quality successive umbrella sampling runs. For the simulations with the standard system geometry, the torus, an exponential increase of $\tau$ with the system volume is seen. In contrast to this, the autocorrelation times obtained for simulations on a sphere are best described by a polynomial increase $\tau \sim V^{\alpha}$ with $\alpha=2.47(10)$, which is distinctively different from the result $\tau \sim V^{2}$ expected for a barrier-free simulation. The apparent elimination of an exponential increase in autocorrelation time is caused by the elimination of the 

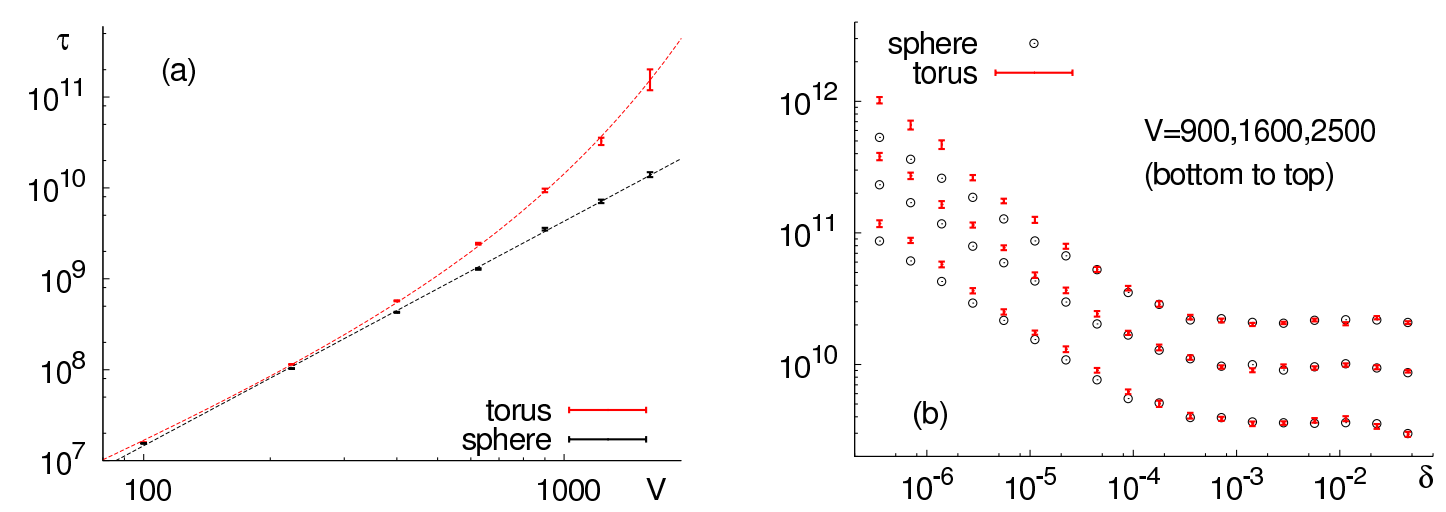

Figure 5.7: Different measures for the increased performance of hypersphere-based simulations of the 2D WR mixture at $z_{\mathrm{A}}=z_{\mathrm{B}}=2.5$. Figure (a) shows the autocorrelation time $\tau$ for a simulation using perfect weights as a function of system volume. For a toroidal system the exponential increase $\tau \sim V^{2} e^{0.0024 V}$ owing to the droplet $\leftrightarrow$ slab transition is seen, whereas spherical systems are best fitted by the polynomial $\tau \sim V^{2.47}$. Figure (b) shows the number of Monte Carlo steps required to finish an iteration of Wang-Landau sampling of said systems as a function of the weight change $\delta$ (cf. 4.2.1). In late iterations of WL sampling where $\delta$ is small, the barrier caused by the droplet $\leftrightarrow$ slab transition becomes effective, requiring an increased amount of Monte Carlo steps to fulfill the flatness criterion.

droplet $\leftrightarrow$ slab transition, which is absent on the sphere.

The reason that even on a sphere the autocorrelation time does not scale proportional to $V^{2}$ is probably caused by nucleation, i.e. the formation of a minority phase droplet. The nucleation barrier is in fact expected to also cause exponential increase in autocorrelation time, albeit a less severe one ${ }^{78}$. The data shown in figure 5.7 , however, are not compatible with an exponential increase, at least not over the whole range of system volumes being considered. The origin of this discrepancy between thermodynamics and simulation result is probably caused by finite-size effect that were not taken into account: simulations of larger systems are expected to ultimately show the exponential increase.

\subsubsection{Significance for Uniform Sampling Strategies}

In most simulations good weights are not available prior to the simulation, and so the autocorrelation time measurement should be considered a generic test. While this test seems to have no direct relevance for the determination of the order parameter distribution it nicely demonstrates the effect of the shape transitions. Also, there is a closely related scenario that can occur in the sampling of OPDs: In late stages of Wang-Landau sampling a good guess for the weights is already available, the change $\delta$ being applied to the weights after each MC step becomes negligible, and the flatness criterion to terminate an iteration requires the simulation to visit the whole range of $N_{\mathrm{A}}$. Figure 5.7(b) shows the number of 
Monte Carlo steps required to finish one iteration of Wang-Landau sampling as a function of the weight change $\delta$. The data were obtained by performing $40 \mathrm{WL}$ simulation runs for each system and each geometry (setup one in table 5.1), and averaging the results. The error bars for the toroidal data reflect the error estimate for this average, error bars for the spherical systems are the same size and not shown.

For early iterations (large $\delta$ ) the results for Wang-Landau sampling times in figure 5.7 show no measurable deviations between the number of Monte Carlo steps required to finish an iteration in either geometry. This is explained by two effects overshadowing the shape transition barrier. First, in early iterations the weights are assumed to be still of bad quality, and so the lack of adequate weights dominate the comparably less severe shape transition barriers. Second, large $\delta$ imply that the weights are dynamic during the iteration, and can push the system over any barrier. In later stages, both these points gradually become less correct, and indeed for $\delta<10^{-4}$ a measurable difference between both geometries develops. This difference becomes more pronounced for additional iterations. But even at $\delta \approx 3.5 \cdot 10^{-7}$ the relative difference is less pronounced than in the autocorrelation times $\tau$ (a factor of 1.6 vs. a factor of 10 for the $V=1600$ system).

The analysis of the autocorrelation time $\tau$ clearly demonstrates the presence of a hidden sampling barrier in the toroidal systems and its absence in spherical systems. But on the scales being taken into account here this does not translate to a faster Wang-Landau simulation, as a single MC step on a sphere requires roughly 1.5 times more CPU time than a single MC step on a square system. On top of this, for successive umbrella sampling no measurable deviation in the number of Monte Carlo steps is expected at all, which raises the question whether in light of these results the use of a spherical geometry is justified. This can indeed be the case, because there is another aspect that needs to be considered, and that is the quality of the data obtained by the simulations.

\subsubsection{Data Quality}

The shape transition barriers not only increase the number of Monte Carlo steps required for a sampling with perfect weights or late stages of Wang-Landau sampling. More importantly, the shape transitions introduce errors to the simulations due to remanence effects. For demonstrating this effect, several independent simulation runs have been performed for both geometries. For any observable $X$, the independent estimates spread around their average with some root mean square width that provides an estimate of the statistical error of an individual measurement. The ratio

$$
Q(X):=\frac{u_{t}(X)}{u_{s}(X)}
$$

is a measure for the quality increase of simulations on a sphere, where $u_{t}(X)$ and $u_{s}(X)$ are the statistical errors in the measurement of observable $X$ on a toroidal and a spherical geometry, respectively. 


\begin{tabular}{c|ccccc}
\multicolumn{5}{c}{ Wang-Landau Sampling (2D) } \\
$\mathbf{V}$ & $Q\left(\left\langle\rho_{A}\right\rangle\right)$ & $Q(\langle|m|\rangle)$ & $Q\left(\left\langle|m|^{2}\right\rangle\right)$ & $Q\left(\left\langle|m|^{3}\right\rangle\right)$ & $Q\left(\left\langle|m|^{4}\right\rangle\right)$ \\
\hline 900 & 1.4 & 1.7 & 1.7 & 3.4 & 1.2 \\
1600 & 2.9 & 3.8 & 3.8 & 6.5 & 3.7 \\
2500 & 2.7 & 3.3 & 3.3 & 4.7 & 7.8
\end{tabular}

Successive Umbrella Sampling (2D)

\begin{tabular}{c|ccccc}
$\mathbf{V}$ & $Q\left(\left\langle\rho_{A}\right\rangle\right)$ & $Q(\langle|m|\rangle)$ & $Q\left(\left\langle|m|^{2}\right\rangle\right)$ & $Q\left(\left\langle|m|^{3}\right\rangle\right)$ & $Q\left(\left\langle|m|^{4}\right\rangle\right)$ \\
\hline 900 & 1.6 & 2.6 & 2.6 & 6.8 & 1.8 \\
1600 & 3.5 & 6.3 & 6.3 & 15 & 2.7 \\
2500 & 4.4 & 11 & 11 & 30 & 11
\end{tabular}

\begin{tabular}{c|ccccc}
\multicolumn{5}{|c}{ Wang-Landau Sampling (3D) } \\
$\mathbf{V}$ & $Q\left(\left\langle\rho_{A}\right\rangle\right)$ & $Q(\langle|m|\rangle)$ & $Q\left(\left\langle|m|^{2}\right\rangle\right)$ & $Q\left(\left\langle|m|^{3}\right\rangle\right)$ & $Q\left(\left\langle|m|^{4}\right\rangle\right)$ \\
\hline 3375 & 1.4 & 1.3 & 1.3 & 1.3 & 1.3
\end{tabular}

Table 5.2: $Q$-values (see equation 5.5) for basic observables of WR mixtures using the simulation and analysis parameters listed in table 5.1. All observables have $Q>1$ for all system sizes, indicating a better data quality for simulations on a sphere. In successive umbrella sampling, the quality loss of simulations on a torus compared to those on a sphere are stronger than in Wang-Landau sampling. This is consistent with the finding that WL simulations on a torus perform a larger number of MC steps than equivalent simulations on a sphere (figure 5.7), whereas SUS samples an equal amount of states on both geometries. 

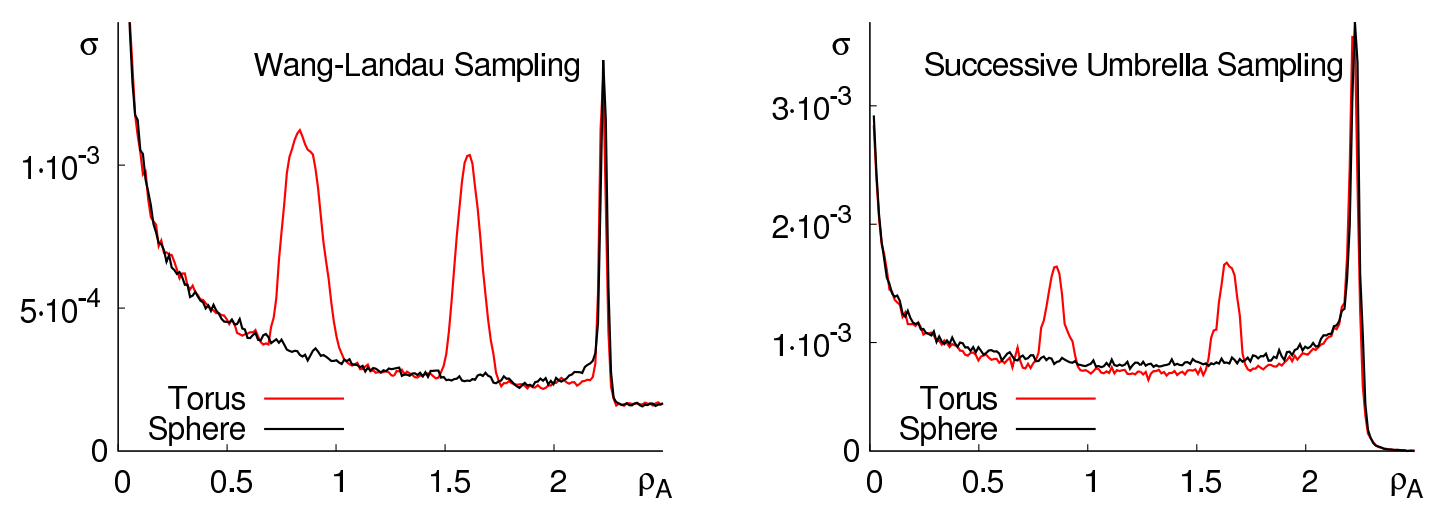

Figure 5.8: Local error estimates $\sigma$ for simulations of a $V=2500$ two-dimensional WR model (setup one of table 5.1) for Wang-Landau and successive umbrella sampling for both geometries.

In table 5.2 the $Q$-values are shown for different system sizes and different basic observables (note that the central moments $\left\langle|m|^{i}\right\rangle$ defined in (3.3) appear in the calculation of the $\chi_{i}$ and the cumulants). The data shown were obtained using the parameters in table 5.1. They clearly show an increased quality for simulations performed on a sphere surface in all of the tested observables. The quality gain obtained by using a spherical system geometry seems more pronounced in successive umbrella sampling where, unlike in WangLandau sampling, an equal number of MC steps is performed for the simulations on both geometries. But even in WL sampling, where simulations on toroidal systems perform extra MC moves compared to simulations on a sphere (c.f. figure 5.7), the results obtained in spherical systems are of better quality.

By considering local quantities, it can be demonstrated that the increase in data quality for simulations on a sphere is indeed caused by the elimination of the transition between droplet and slab shape. To this end, the weights $w\left(N_{\mathrm{A}}\right)$ obtained from the simulations have been considered. For each $N_{\mathrm{A}}$, each simulation provides an estimate for the local weight change $w\left(N_{\mathrm{A}}+1\right)-w\left(N_{\mathrm{A}}\right)$. As in the case of the observables, the 40 values obtained for the local weight change spread around some average value with a root mean square $\sigma\left(N_{\mathrm{A}}\right)$. These uncertainties in the local weight change are shown in figure 5.8, where the $\sigma\left(N_{\mathrm{A}}\right)$ have been averaged over 30 bins to provide a clearer signal. In both sampling methods, the simulations performed on toroidal systems show peaks in the weight uncertainty $\sigma$ at the density where the transition between droplet and slab occurs. In the simulations performed on a hypersphere these peaks are absent. Since apart from these two peaks the errors in the local weight are the same for both geometries this demonstrates that the quality difference seen in table 5.2 is indeed caused by the elimination of the shape transition. The peak at $\rho_{\mathrm{A}} \approx 2.2$ seen in both geometries can be identified as being caused by the nucleation transition, which is still present in spherical systems, and has already been indicated by the measurement of the autocorrelation time (figure 5.7). The peak for the nucleation of an A-phase droplet would be expected at very 

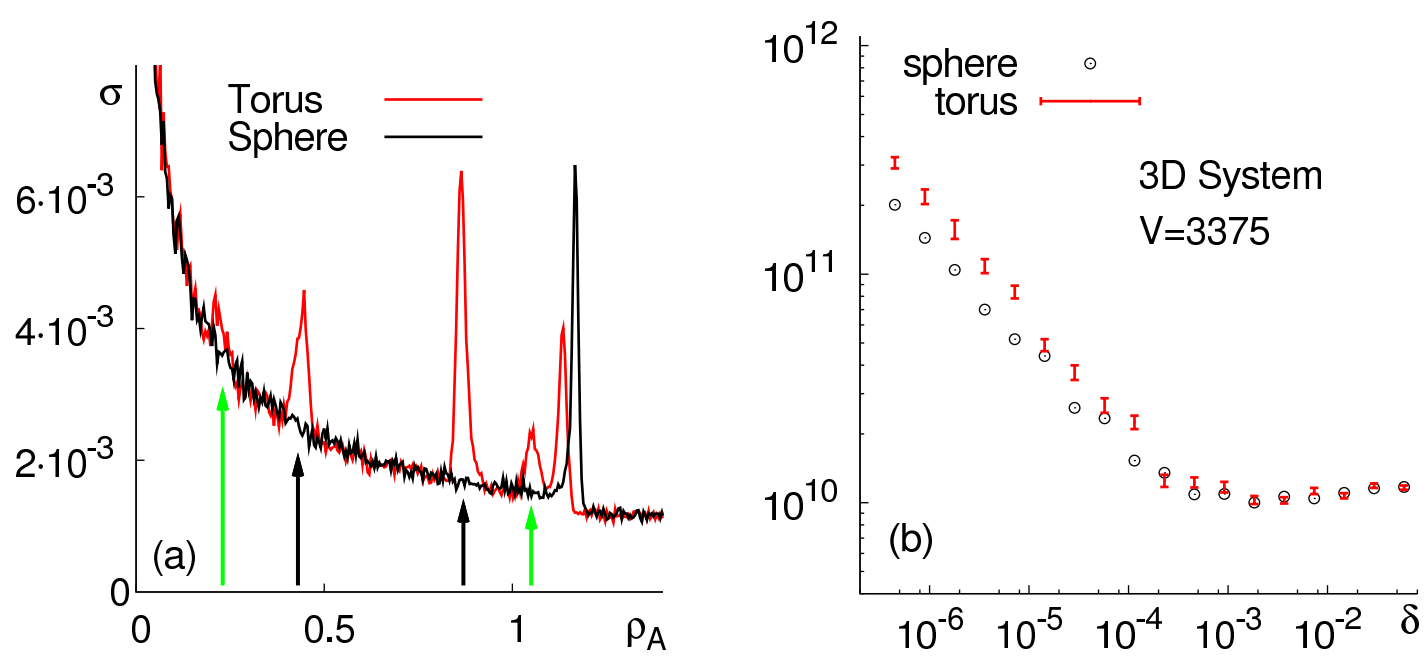

Figure 5.9: Wang-Landau sampling results for a 3D WR mixture with $V=3375$ being simulated on the standard toroidal geometry and the surface of a four-dimensional hypersphere. In figure (a), the uncertainties $\sigma$ of the local weights are shown. For the standard cubic system they exhibit peaks that are attributed to nucleation and to the shape transitions between droplet and stalk (green arrows) and between stalk and slab (black arrow). Such peaks are not seen for the spherical geometry, indicating the absence of shape transitions. Figure (b) shows the number of MC steps required to finish an individual iteration of Wang-Landau sampling. As in the two-dimensional case (figure 5.7) the absence of shape transitions causes late iterations (with small weight changes $\delta)$ to fulfill the flatness criterion faster.

low $\rho_{\mathrm{A}}$. It is not clear whether the large $\sigma$ values seen there overshadow this peak, or if they actually are the nucleation peak.

\subsubsection{The 3D Case}

Contrary to the two-dimensional case, the three-dimensional WR model on the surface of a four-dimensional hypersphere is not easily visualized. So while the absence of shape transitions in 2D systems being simulated on the surface of a sphere is already quite apparent from a visualization of states (figure 5.5), it is not obvious that there are no shape transitions of the minority phase in $3 \mathrm{D}$ systems simulated on the surface of a $4 \mathrm{D}$ hypersphere. But building upon the findings in figure 5.8 the absence of shape transitions in this case can be inferred indirectly from investigating the local uncertainties $\sigma\left(N_{\mathrm{A}}\right)$ and demonstrating the absence of barriers in these data. To this end, Wang-Landau simulations have been performed on both geometries with the parameters listed in table 5.1 .

As shown in figure 5.9(a), the local uncertainties extracted from the simulations in a 3D cube with periodic boundary conditions show peaks that can be attributed to the shape 
transitions of the minority phase between droplet, stalk, and slab and the nucleation of a minority phase droplet. Contrary to the $2 \mathrm{D}$ case the nucleation peak is not identical in both geometries. The origin of the discrepancy of the nucleation peaks between the two geometries has not been investigated in detail, but it probably stems from the $V=3375$ spherical system with $a=1$ not being an exact equivalent of the $L=15 \mathrm{WR}$ system (cf. section 5.2.1.1).

The peaks attributed to the shape transitions in the cubic system are absent in the results for simulations on the surface of a four-dimensional hypersphere. This is an indicator that shape transitions of the minority phase are indeed eliminated. Again, the absence of shape transitions results in an improved sampling efficiency, as seen in figure 5.9(b), where it is shown that on the surface of a hypersphere the late iterations of the Wang-Landau sampling require less MC moves to fulfill the flatness criterion. Finally, the $Q$-values shown in table 5.2 also indicate a quality improvement of the sampling results, as again $Q>1$ for all observables.

\subsection{Other Models on a Sphere}

The simulation of spherical system geometries may appear very artificial - experiments are usually not performed on the surface of a three or even four-dimensional sphere, except for some cases like experiments on membrane vesicles ${ }^{81 ; 82 ; 83 ; 84}$. This is, however, not necessarily an argument in favor of the standard geometry choice given that experiments are not conducted on cubes with periodic boundary conditions, either.

Considering the increase in sampling performance of the WR mixture on a hypersphere motivates the question which other models may be applicable for a transfer to a hypersphere. Clearly, the most natural candidates are off-lattice models, since grids suffer from connectivity defects when transferred onto the surface of a hypersphere. For the same reason, crystalline phases cannot be represented. Also, models with non-spherical particles and an interaction that depends on the shape of the particles are probably unsuitable, such as the two-dimensional Zwanzig model (see section 2.1.4). The exception are models where there is no coupling between the spatial and the orientational degrees of freedom ${ }^{85}$.

From a computational point of view, some models may be unsuitable for a transfer to a sphere because of an unacceptable increase in CPU time per Monte Carlo step. In the WR model, the use of CPU time intensive trigonometric functions could be avoided. But this may not be true for all models, particularly not those where the pair-potential continuously changes with the distance of the particles (note that if the pair potential is a step function over only a few intervals one can find the correct interval via a few threshold values $\left.a_{1}, a_{2}, \ldots\right)$. In models with long-ranged Coulomb interactions, it has been proposed that the use of a spherical geometry can be beneficial for the calculation 
5.3. OTHER MODELS

of forces ${ }^{86}$. 



\section{Random Neutral Obstacles}

In this chapter, the simulation methods of the preceding chapters are used to study the effect of quenched obstacles on the phase separation in binary mixtures with short ranged interactions. To add quenched disorder to the Widom Rowlinson mixture, a third particle species $\mathrm{O}$ (obstacles) of immobile unit diameter spheres is added. The obstacles are chosen to have a hard-core repulsion with both mobile species A and B. Since the obstacles' interaction is indifferent to the mobile species, this choice of obstacles is the case of neutral obstacles. It preserves the $\mathrm{A} \leftrightarrow \mathrm{B}$ symmetry of the WR model, which greatly simplifies the data analysis because a phase transition between $\mathrm{A}$ and $\mathrm{B}$ phase is then known to lie on the line $z_{\mathrm{A}}=z_{\mathrm{B}}$. The exact mapping of the mixture picture onto a fluid model (section 2.1.2) is destroyed, however. The scenario of random neutral obstacles presented here is a model of a symmetric binary mixture with short-ranged interactions, but not a model of a generic fluid in the presence of quenched disorder.

Since the WR model is in the universality class of the Ising model, the WR model with this kind of random obstacles that exclude an equal amount of volume for both particle species can be expected to behave like the Ising model with random site dilutions. Above a certain threshold amount of dilution, the percolation threshold, no long range magnetic order can exist in such an Ising model, and therefore no distinguished A and B phase would be expected for the WR model. It is predicted in renormalization group theory that for low amounts of dilutions the site-diluted Ising model still has a phase transition between a ferromagnetic and a paramagnetic state with a renormalization group flow towards the Ising model's critical point at zero dilution ${ }^{6}$. However, this Ising universality of the critical point is subject of debate ${ }^{87 ; 88}$. If the universality is that of the pure Ising model logarithmic corrections have to be taken into account for system sizes that are computationally accessible ${ }^{89}$. Agreement with pure Ising universality after taking

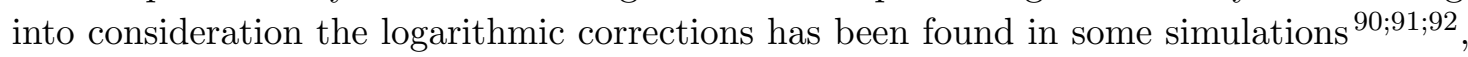
whereas in other simulations no agreement with pure Ising universality was found despite those corrections ${ }^{89 ; 93}$.

In this work the case of interest is that of low obstacle density $\rho_{\mathrm{O}}$, which is measured in terms of the average number of obstacles per unit volume, and with a focus on the fate of the phase transition from an A phase to a B phase. The presence of randomly located static obstacles introduces a new layer of complexity that needs to be considered: While a thermodynamic system may have a well-defined obstacle density, randomly selected small 
pieces of this system (the necessarily finite system that one can simulate) do not have exactly this number of obstacles per unit volume. Instead, in a randomly-picked volume $V$ of the system there is a random number of obstacles that is distributed according to a Poisson distribution with mean $\rho_{\mathrm{O}} V$. Furthermore, the behavior of the mixture in such a finite system generally not only depends on the number of obstacles, but also on their locations. To take this into account simulations have been performed for many obstacle configurations, and their results have been suitably averaged over (as described in section 6.2). Compared to pure systems the investigation of systems with quenched disorder therefore adds an additional layer of complexity, and is computationally more demanding.

\subsection{Simulation}

The simulations of each individual obstacle configuration is performed in the following way: First, a random number of obstacles $N_{O}$ is drawn from a Poisson distribution with mean $\rho_{\mathrm{O}} V$, where $\rho_{\mathrm{O}}$ is the obstacle density of the infinite-sized thermodynamic system. Then, the $N_{\mathrm{O}}$ obstacles are placed at random locations inside the empty system volume, whereby overlaps between $\mathrm{O}$ particles are allowed. After generating a starting state in which the $\mathrm{A}$ and the $\mathrm{B}$ particles are equilibrated, the system is then being simulated via successive umbrella sampling (section 4.2.2) using the single insertion and removal steps described in section 4.2 - with the additional rule that insertions of A or B particles that would lead to an intersection of the newly-inserted particle with an obstacle are always rejected.

When $N_{\mathrm{A}}=0$, the B particles are an ideal gas in the system volume not excluded by the presence of the obstacles. Consequently, the initialization routine described in section 4.2.2 can still be used for the construction of an initial state with only the minor adaption that insertions of B particles that would intersect with an obstacle are discarded. An exact analytical solution of the first SUS window is not possible when obstacles are present. But if the amount of obstacles is not too large, the analytic result for the pure model (equation 4.14) is an adequate guess for a simulation weight in the first window.

\subsection{Disorder Average}

Since the configuration of the obstacles differ from simulation to simulation, so do the resulting order parameter distribution (see figure 6.1). The simulation of many different obstacle configurations therefore does not yield a single order parameter distribution $P_{L}$ as in the pure model, but a sequence $P_{L, i}$ of OPDs, where $i=1 \ldots N$ is the sequence index. Of course, not only the OPD but also the thermal averages of observables can depend on the configuration of the obstacles, i.e. one also has $\langle\cdot\rangle \rightarrow\langle\cdot\rangle_{i}$. This motivates the following definition: Let $O_{i}$ be the result for an observable $O$ that has been evaluated 

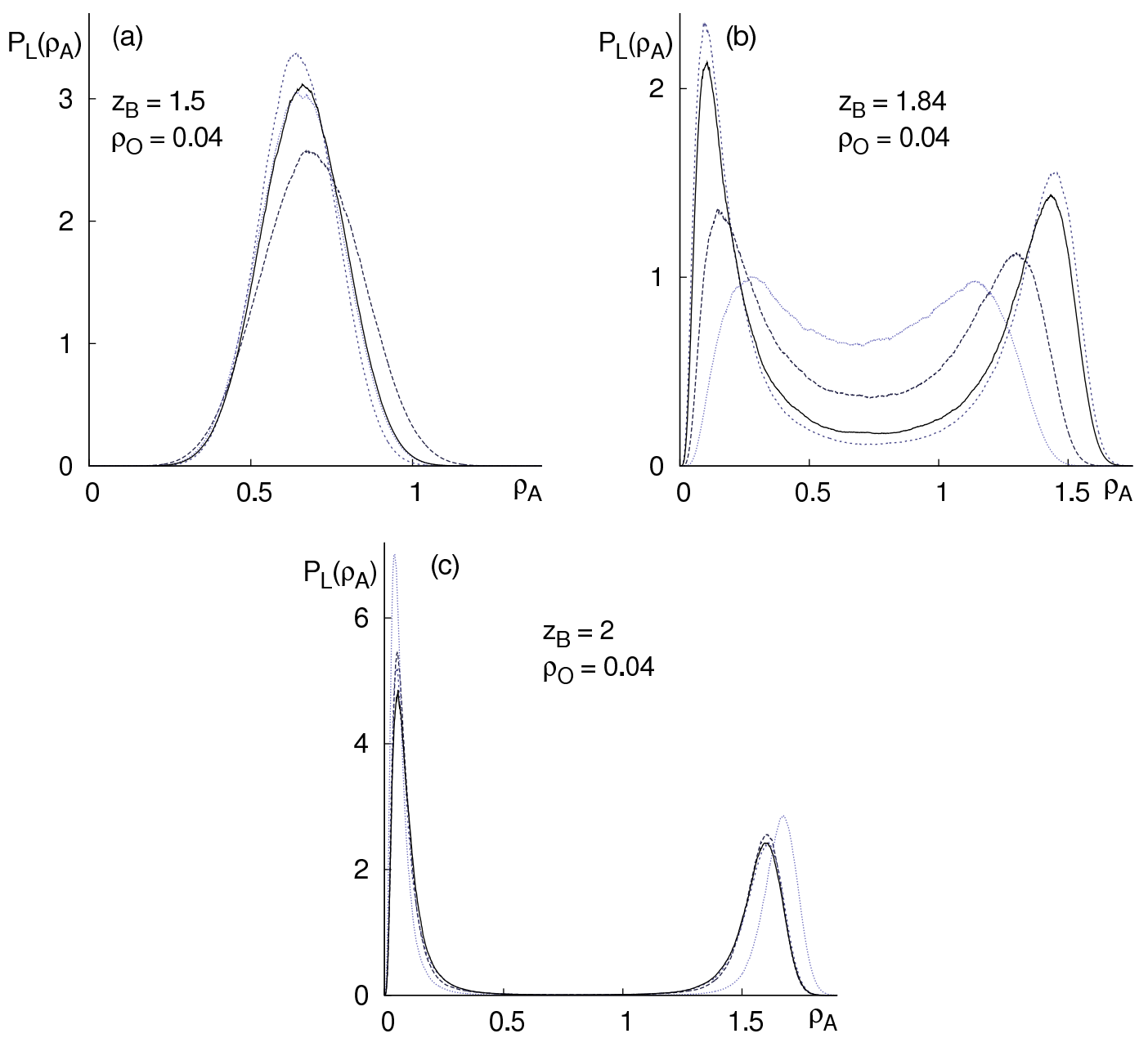

Figure 6.1: Randomly-picked order parameter distributions for different configurations of obstacles in a $30 \times 30 \mathrm{WR}$ system on the $l_{\mathrm{MS}}$ locus below (figure a), around (b), and above the critical fugacity (c). The distributions for different obstacle configurations all qualitatively show the same behavior as the pure model but differ quantitatively. This configuration-dependence of the OPD necessitates introducing the disorder average to determine the behavior of the system in the thermodynamic limit. 
from the $i$-th of a total of $N$ mutually independent random obstacle configurations. Then, the average

$$
[O]:=\frac{1}{N} \sum_{i=1}^{N} O_{i}
$$

is the disorder average of that observable.

The disorder average constitutes an additional average over the thermal one that must be taken into account for the data analysis. The natural extension for the finite-size scaling of the pseudocritical points inferred from the extrema of $\chi_{2}, \chi_{3}$, and $\chi_{4}$ is extrapolating the extrema of their disorder averages $\left[\chi_{2}\right],\left[\chi_{3}\right]$, and $\left[\chi_{4}\right]$. The proper equivalent of the cumulants is ambiguous. The Binder cumulants can be considered as a measure of supercriticality of an individual system, where values $U_{1} \approx \pi / 2$ indicates supercriticality and $U_{1} \approx 1$ indicates two coexisting phases. With this interpretation of the Binder cumulant one may consider

$$
\left[U_{1}\right]=\frac{1}{N} \sum_{i=1}^{N} U_{1, i},
$$

where $U_{1, i}$ is the cumulant value measured for the $i$-th system. On the other hand, the second and the first central moment of the OPD that appear in the nominator and the denominator of the cumulant definition in (3.20) can also be considered as the observables, which motivates the definition

$$
U_{1}=\frac{\left[\left\langle\left|\rho_{\mathrm{A}}-\left\langle\rho_{\mathrm{A}}\right\rangle\right|^{2}\right\rangle\right]}{\left[\left\langle\left|\rho_{\mathrm{A}}-\left\langle\rho_{\mathrm{A}}\right\rangle\right|\right\rangle\right]^{2}}
$$

Further averages like averaging over susceptibility and order parameter independently are also conceivable, and there is no unique correct way to perform the disorder average. The choices being made here are to some extend arbitrary, but lead to similar conclusions as demonstrated in figure 6.3.

For any configuration of symmetric obstacles it is clear that the transition from the A to the $\mathrm{B}$ phase can only occur at $z_{\mathrm{A}}=z_{\mathrm{B}}$, which may hence seem to be the locus of choice. Despite this, for a given $z_{\mathrm{B}}$ each OPD has been reweighted to the $l_{\mathrm{MS}}$ locus, instead. In the context of symmetric obstacles this should be understood as a trick to compensate for sampling errors, especially those caused by the shape transitions described in chapter $5^{\mathrm{a}}$.

\subsection{Results}

The first indication for the behavior of the system is the visual inspection of system snapshots and a few order parameter distributions at different $z_{\mathrm{B}}$. A few randomly

\footnotetext{
${ }^{a}$ The individual tuning of the OPDs to some locus does have a physical significance for asymmetric obstacles, though. This is discussed in section 7.1.1.
} 

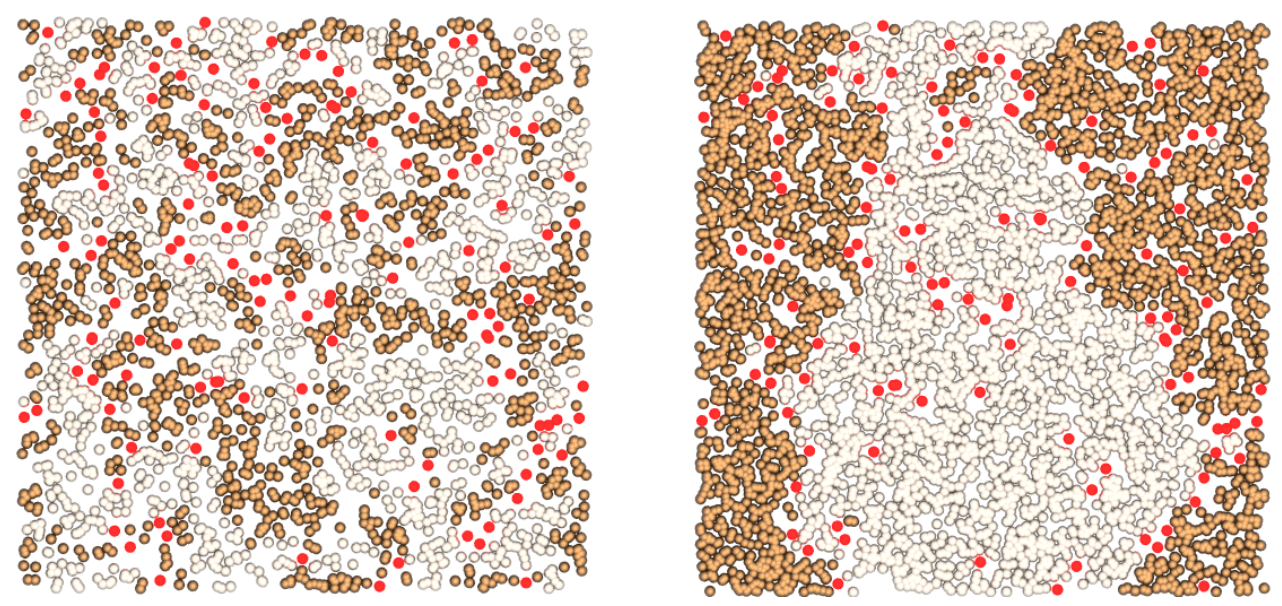

Figure 6.2: Snapshots of the 2D WR mixture with $\rho_{\mathrm{O}}=0.05$ neutral obstacles in the microcanonical ensemble show mixed state at low densities $\rho_{\mathrm{A}}=\rho_{\mathrm{B}}=0.5$ (left image) and a demixed state at high densities $\rho_{\mathrm{A}}=\rho_{\mathrm{B}}=1$ (right image) indicating the existence of a demixing transition. The neutral obstacles are drawn in red.

selected obstacle configurations reveal that the order parameter distributions at high $z_{\mathrm{B}}$ (and on the $l_{\mathrm{MS}}$ locus) tend to have well-distinguishable liquid and gas peaks, while at lower $z_{\mathrm{B}}$ they feature only a single peak corresponding to a mixed phase. Close to a pseudocritical point, the OPDs resemble that of the critical distribution of the pure model, except that the exact shape depends on the obstacle configuration (see figure 6.1). Visual inspections of microcanonical simulations also indicate the existence of the qualitatively same phase transition as in the pure model: As shown in figure 6.2 states at low densities tend to be mixed, while at high densities the A and B particles are demixed. Based on those simple observations a finite-size scaling analysis using the disorder averaged quantities can be applied as normal.

\subsubsection{Finite-size scaling}

The expected universality class, the diluted Ising model, features a phase transition with a critical point for which hyperscaling holds. Therefore, a cumulant intersection should be found at this critical point. In figure 6.3 the cumulant intersection plots for the disorder averaged cumulants according to equations 6.3 (figure a) and 6.2 (figure b) for an obstacle density $\rho_{\mathrm{O}}=0.04$ are shown. The curves all drop from the single-peak value $U_{1}=\pi / 2$ to the value $U_{1}=1$ associated with two independent peaks, and intersect at $z_{\mathrm{B}}=1.915(10)$ and $z_{\mathrm{B}}=1.910(7)$ for $U_{1}$ and $\left[U_{1}\right]$, respectively. This indicates a critical point $z_{\mathrm{B}, \mathrm{cr}}$ at this value, and the existence of an $\mathrm{A}$ and $\mathrm{B}$ phase above it. As both estimates for a disorder averaged cumulants effectively give the same results, both definitions are adequate for the task. 

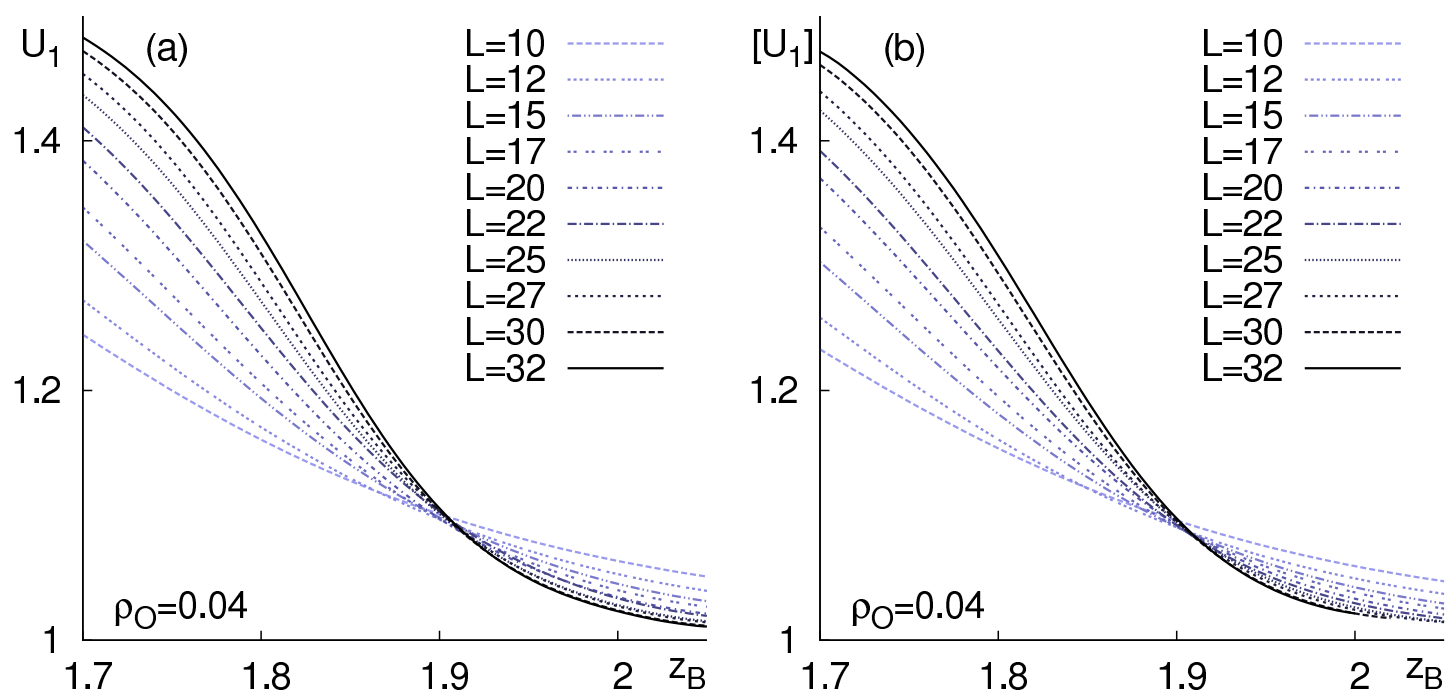

Figure 6.3: Cumulant plots for the 2D WR model with $\rho_{\mathrm{O}}=0.04$ neutral obstacles. Both definitions of the disorder averaged cumulant, $U_{1}$ according to (6.3) in figure (a) and $\left[U_{1}\right]$ according to (6.2) in figure (b) have been taken over the same 600 obstacle configurations for each system size. The curves obtained from the two different configuration averages are almost identical, and predict a critical point at $z_{\mathrm{B}, \mathrm{cr}}=1.915(10)\left(U_{1}\right)$ and $z_{\mathrm{B}, \mathrm{cr}}=1.910(7)\left(\left[U_{1}\right]\right)$.

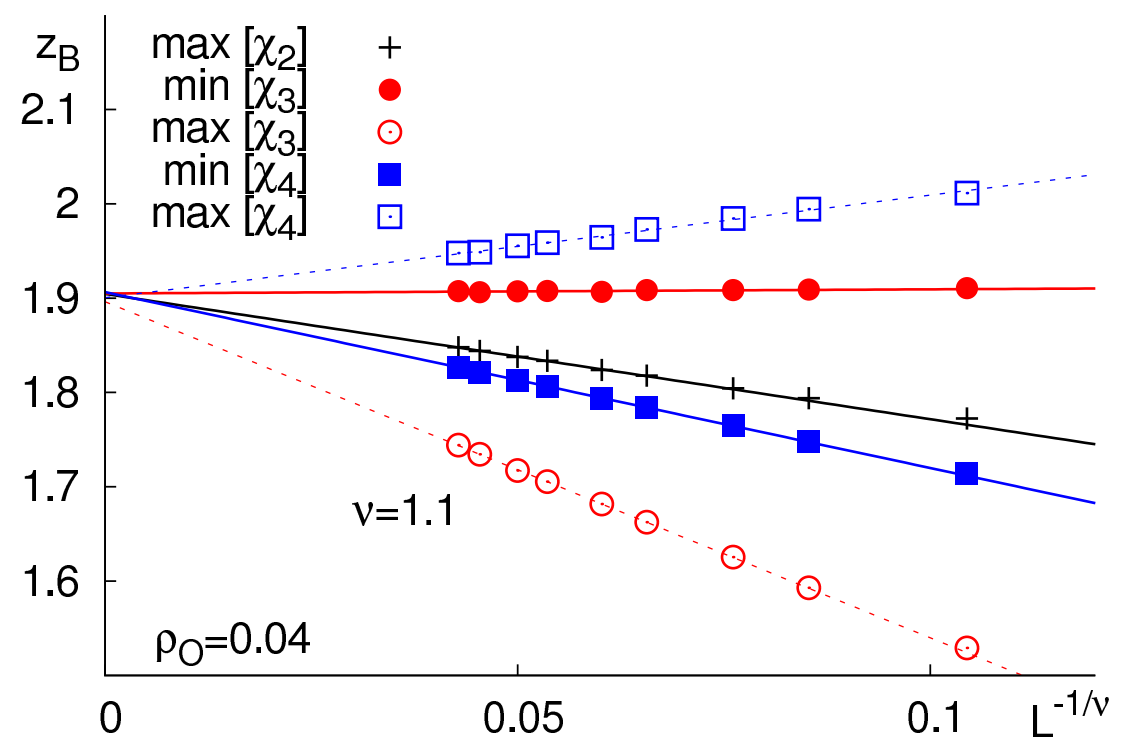

Figure 6.4: Finite-size scaling of the extrema in $\left[\chi_{i}\right]$. The different pseudocritical point scale to a common critical point for $\nu=1.1(1)$, which is approached from above and from below. For $\nu=1.1$ the critical fugacity $z_{\mathrm{B}, \mathrm{cr}}=1.904(4)$ is found. 
In figure 6.4 the finite-size scaling of the global extrema of the observables $\left[\chi_{2}\right],\left[\chi_{3}\right]$, and $\left[\chi_{3}\right]$ according to equation 3.17 is shown for the same obstacle density. The scaling lines for the different observables nicely intersect for a correlation length exponent $\nu=1.1(1)$. This value for $\nu$ and its uncertainty have been inferred from visual inspection of the plots and eye-gauging values that result in good agreement between the curves. It must be emphasized that the value is to be understood as a best-fit parameter, not as a measurement of the true correlation length exponent. The analysis setup introduced in section 3.2 and used throughout this work does not take into account possibly important logarithmic corrections. The focus of the analysis is the proof of a demixing transition via location of a critical point, not a precision measurement of the critical exponents ${ }^{\mathrm{b}}$. The values presented for $\nu$ should thus be understood as an effective correlation length exponent, only.

With $\nu=1.1$ the location of the critical point is $z_{\mathrm{B}, \mathrm{cr}}=1.904(04)$. This result is rather insensitive to $\nu$, because the location of the minimum of $\left[\chi_{3}\right]$ happens to be almost independent of the system size. As in the pure model, the pseudocritical points approach $z_{\mathrm{B}, \mathrm{cr}}$ from above and from below, which puts a strong $\nu$-independent constraint on the possible location of $z_{\mathrm{B}, \mathrm{cr}}$.

\subsubsection{Self-averaging}

As demonstrated by the results in figure 6.3 and 6.4 the disorder-averaged observables $U_{1}$, $\left[U_{1}\right]$ and $\left[\chi_{i}\right]$ all predict a critical point at somewhat higher inverse temperature compared to the pure model. But for the large thermodynamic system this only implies a well defined configuration-independent critical point if the system self-averages, that is if the differences between different obstacle configurations vanish as the system size approaches the thermodynamic limit. To demonstrate that this is indeed the case, a pseudocritical point has been determined for each individual configuration by locating the maximum of $\chi_{2}$, and the scatter around the average pseudocritical fugacity has been investigated.

In the left plot of figure 6.5 a histogram of pseudocritical points for two different system sizes is shown. The histograms clearly show that the pseudocritical fugacities $z_{\mathrm{cr}, i}$ differ between obstacle configurations. As the system size is increased, the root mean square width $\sigma\left(z_{\mathrm{cr}, i}\right)$ of the histograms becomes smaller, indicating that indeed $\sigma\left(z_{\mathrm{cr}, i}\right) \stackrel{L \rightarrow \infty}{\longrightarrow} 0$. This decrease is shown in the right plot of figure 6.5 , where the $\sigma\left(z_{\mathrm{cr}, i}\right)$ are shown for a range of system sizes and a number of obstacle densities. For an effective correlation length exponent $\nu=1.2(1)$ the decrease is in excellent agreement with the scaling prediction ${ }^{94}$

$$
\sigma\left(z_{\mathrm{cr}, i}\right)=L^{-1 / \nu} \cdot \text { const. }
$$

In the thermodynamic limit $L \rightarrow \infty$, all randomly-constructed obstacle configurations

\footnotetext{
${ }^{\mathrm{b}}$ Considering that there is controversy even in the computationally much simpler site-diluted Ising model the prospects of such an endeavor do not look very promising, anyways.
} 

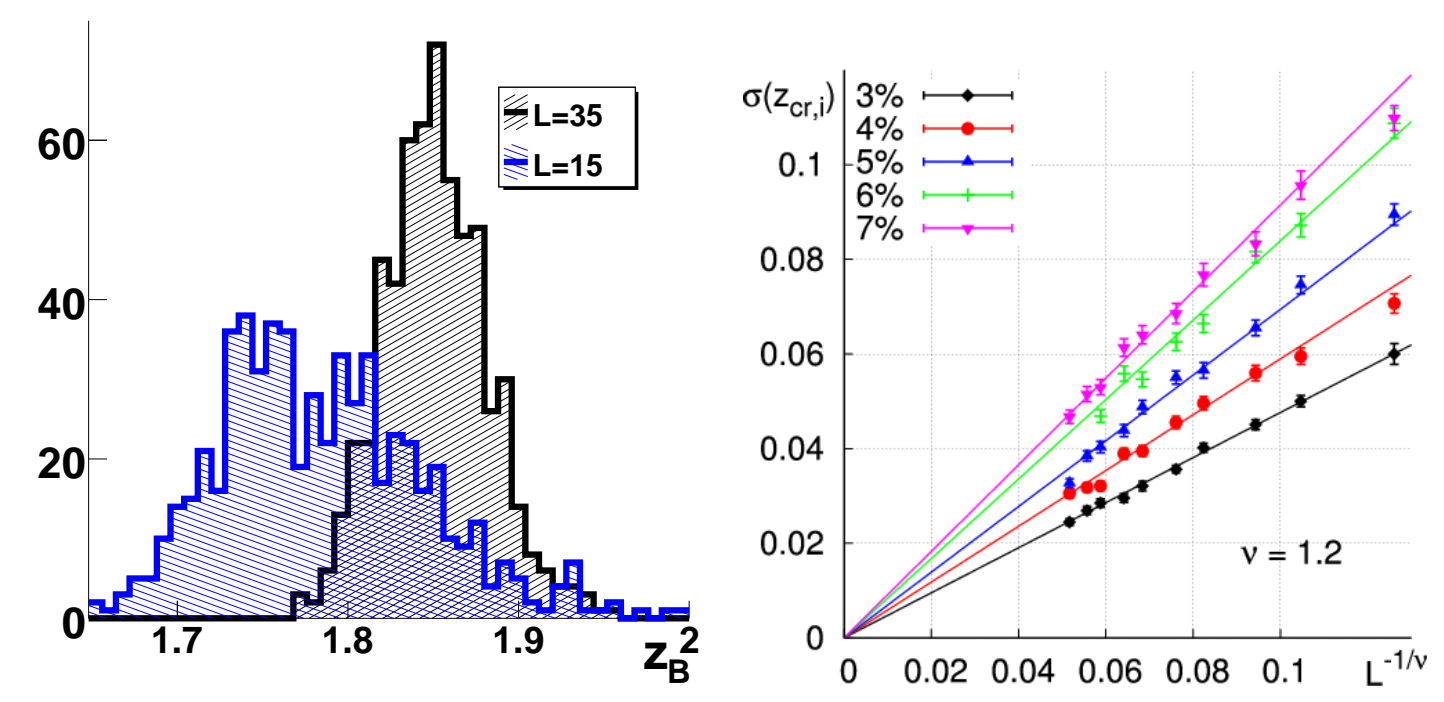

Figure 6.5: Configuration-dependence of the pseudocritical points. The left diagram shows histograms of the pseudocritical points for 600 randomly-generated obstacle configurations with $\rho_{\mathrm{O}}=0.04$. The right plot shows the widths $\sigma\left(z_{\mathrm{cr}, i}\right)$ of these histograms for different system sizes and different obstacle densities. For all $\rho_{\mathrm{O}}$ shown the data are in excellent agreement with the scaling relation (6.4), implying that for $L \rightarrow \infty$ the histograms shown in the left plot become sharp.

hence show the behavior of the disorder average. This implies that in all of the systems there exists a phase transition between an $\mathrm{A}$ and a $\mathrm{B}$ phase, but with a critical point that is at a lower temperature. Qualitatively, the phase diagrams of the WR model with low concentrations of randomly placed neutral obstacles still resembles that of the pure WR model (see figure 2.3). It only depends on the concentration of obstacles, but not on the particular obstacle configuration. This holds true as long as obstacle configurations are randomly-picked from the ensemble of randomly-distributed obstacles. Special "hand-crafted" choices can actually exhibit a different behavior, as is readily seen in cases where all obstacles sit in the same location. But as seen from the vanishing width of the distribution of pseudocritical points, such exotic configurations become rare and insignificant in the thermodynamic limit.

\subsubsection{Effect of the Obstacle Density}

The shift towards higher critical fugacities becomes larger as $\rho_{\mathrm{O}}$ is increased. This is shown in figure 6.6(a) where the critical fugacities are shown as a function of the obstacle density. The estimates for the critical fugacities are a combined estimate from intersections in $U_{1}$ (as in figure 6.3a) and the finite-size scaling of the extrema of the $\left[\chi_{i}\right]$ (as in figure 6.4). For each obstacle density and for each system size, 600 obstacle configurations were 

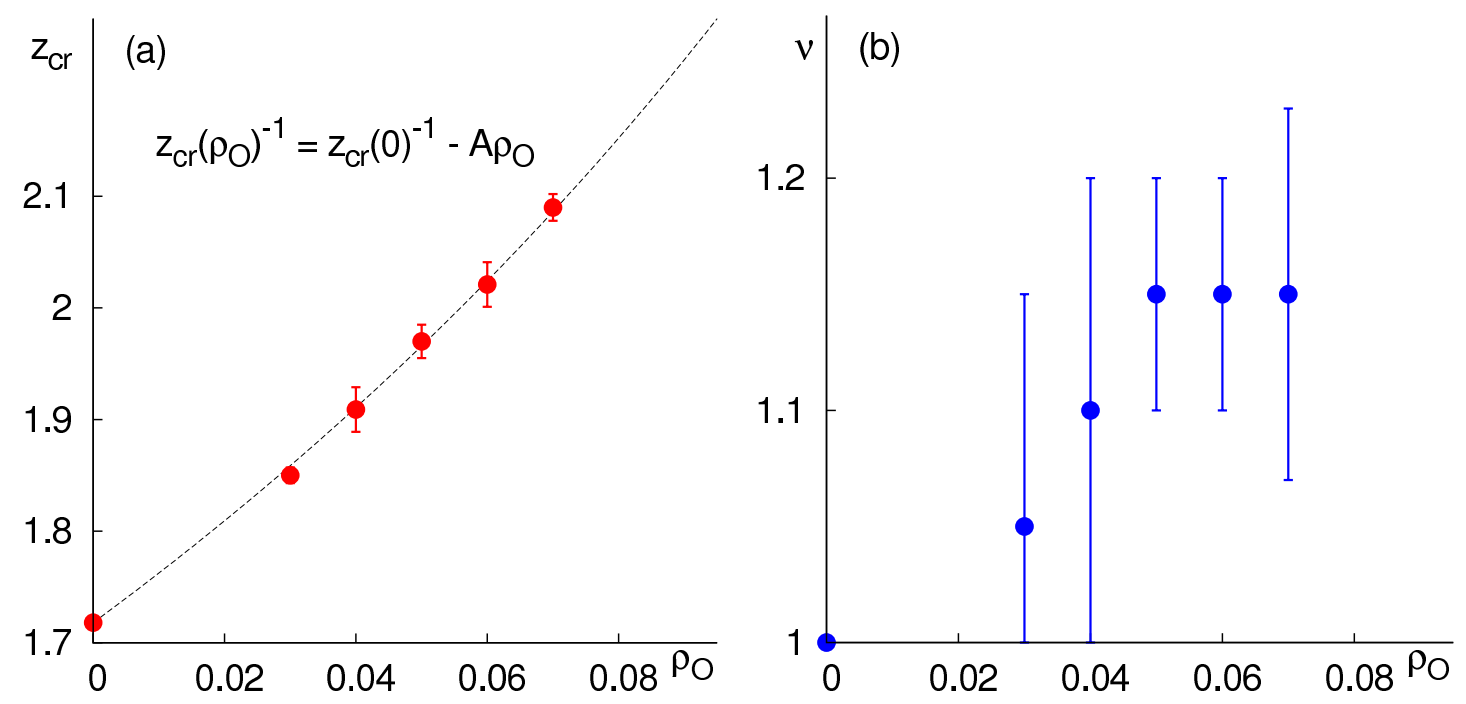

Figure 6.6: Estimated critical point and suitable correlation length exponents as a function of the obstacle density (plots (a) and (b), respectively). The increase in $z_{\mathrm{B}, \mathrm{cr}}\left(\rho_{\mathrm{A}}\right)$ is in good agreement with the prediction in (6.5), as indicated by the best-fit curve. In figure (b), the best-fitting values for the effective correlation length exponent $\nu$ are shown. Values and error bars represent the regions over which the individual maxima in $\left[\chi_{i}\right]$ extrapolate reasonably well to a common critical fugacity (as in figure 6.4).

simulated and averaged over. For the site-diluted Ising model it has been observed that for low concentrations of disorder the decrease in critical temperature is proportional the dilution concentration ${ }^{88 ; 95}$. When $z_{\mathrm{B}}$ is interpreted as an inverse temperature and $\rho_{\mathrm{O}}$ as the concentration of dilution this implies that

$$
z_{\mathrm{B}, \mathrm{cr}}\left(\rho_{\mathrm{O}}\right)=\frac{1}{\frac{1}{z_{\mathrm{B}, \mathrm{cr}}(0)}-A \cdot \rho_{\mathrm{O}}}
$$

with some fit parameter $A$, and $z_{\mathrm{B}, \mathrm{cr}}(0) \approx 1.718$ being the critical fugacity of the pure WR model. For the best-fitting $A$ this prediction is in excellent agreement with the simulation results, as shown by the curve in figure 6.6(a).

Figure 6.6(b) shows the value of the effective correlation length exponent $\nu$, which is inferred from the finite-size scaling of the $\left[\chi_{i}\right]$. The increase of $\nu$ with the obstacle density is compatible with an actual increase of the correlation length exponent with obstacle density ${ }^{87}$, but also with the scenario of an Ising critical point and logarithmic corrections that become more relevant as the amount of disorder is increased. 


\subsubsection{Summary of Results}

In the thermodynamic limit the Widom Rowlinson model with a low density of randomly placed neutral obstacles has a critical fugacity above which a first order transition from an A phase to a B phase can occur. The existence of such a critical fugacity as well as its increase with the amount of obstacles is compatible with the expectation that the system belongs to the universality class of the site-diluted Ising model. This has also been claimed from simulations of a similar three dimensional model ${ }^{96}$.

Because of the pure WR model being a model for a fluid it is tempting to conclude that also for a fluid in the presence of low concentrations of randomly-placed neutral obstacles the same results should hold true, i.e. a qualitatively identical liquid $\leftrightarrow$ gas transition but with a critical point shifted towards lower temperature. However, there are two caveats that one should be aware of: First, the exact mapping of the WR mixture on the WR fluid is destroyed by the presence of the obstacles since the argument that allows to integrate out the B particles, namely that they form an ideal gas in the volume not forbidden by the A particles, is not valid anymore. Second, it is not clear what the physical realization of obstacles that have an identical preference for liquid and gas phase of a fluid might be. In reality one would expect that obstacles do have at least some preference for either phase. It will be shown in the next two chapters that this drastically changes the results obtained for the neutral obstacles. 


\section{D Mixtures with Asymmetric Obstacles}

In reality it is unlikely that obstacles have an identical interaction with both mixture species or (in the fluid picture) an equal affinity to the liquid and the gas phase. Generally, some preference for either species or phase is to be expected. Such obstacles would cause spatially random preferences for either particle species. In an Ising-like analogue such a model may be conceived as an Ising model where random magnetic fields act on each spin, thus creating a local preference for either spin orientation - the random-field Ising model $^{7 ; 8}$ (RFIM). Because of this analogy it has been hypothesized by de Gennes that a fluid in the presence of random obstacles should be in the universality class of the random-field Ising model $^{13}$.

Usually, when considering the RFIM one considers random fields with no correlation between the sites and with the random field on each site being drawn from either a Gaussian distribution centered around zero or from a bimodal distribution. The case of static obstacles is better represented by a more exotic realization of an RFIM, namely one where the random field on each site is zero in most cases, but for a small number of sites is infinitely strong (and thereby fixes the orientation of the spins at these sites). For the RFIM it is known that in two dimensions there cannot be long-ranged orientational $\operatorname{order}^{97}$, whereas in three dimensions a spontaneous magnetization exists at a finite temperature $^{97 ; 98}$. Consequently, the existence of an $\mathrm{A}$ and a $\mathrm{B}$ phase is expected for the WR mixture in $d=3$ dimensions and at sufficiently high fugacities. In this chapter the three-dimensional Widom-Rowlinson model in the presence of random obstacles with an asymmetric coupling to the A and B particles is investigated. As expected, evidence of a phase transition is found, but with very special critical behavior that is characteristic of random-field Ising universality.

\subsection{Simulation}

For the simulations using asymmetric quenched disorder in three dimensions two species of obstacles have been used, both with an equal density $\rho_{\mathrm{O}}$. The $B$-affine obstacles have a hard-core interaction at distance one with the A particles but may freely overlap 

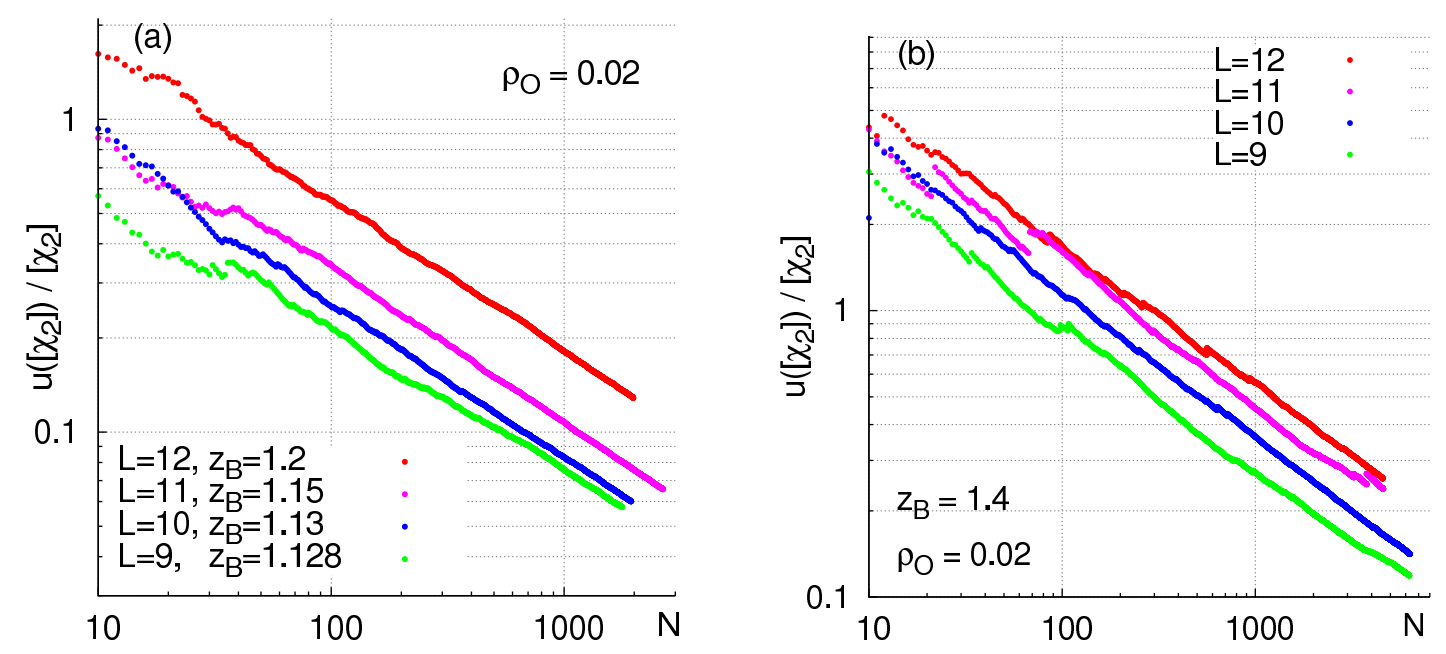

Figure 7.1: The relative uncertainty of $\left[\chi_{2}\right]$ as a function of the number of obstacle configurations $N$ being taken into account to calculate the disorder average. The uncertainty $u\left(\left[\chi_{2}\right]\right)$ is estimated using the Jackknife error method ${ }^{53}$. Figure (a) and (b) show the relative uncertainty of $\left[\chi_{2}\right]$ in the vicinity of the pseudocritical fugacities (i.e. around the maxima of $\left[\chi_{2}\right]$ ) and the fugacity of the critical point in the thermodynamic limit, respectively. In both cases (and also for the other observables) at least a few thousand configurations must be considered to obtain an adequate disorder average.

with the B particles. The $A$-affine obstacle species has an "opposite" interaction, i.e. a hard-core repulsion with the B particles an no interaction with A particles. This choice again prevents an exact mapping of the WR mixture on a fluid, but preserves the $\mathrm{A} \leftrightarrow \mathrm{B}$ symmetry in the average over all possible obstacle configurations. As usual, cubes of different side-lengths $L$ and periodic boundary conditions have been used for the simulation.

Rather than allowing for a Poissonian fluctuation in the number of obstacles, a nearconstant amount of obstacles has been used. For both obstacle species the number of obstacles chosen to represent an obstacle density $\rho_{\mathrm{O}}$ has been chosen at random from the two natural numbers around $\rho_{\mathrm{O}} V$ (which itself usually is not an integer) such that the average number of obstacles over all realizations is $\rho_{\mathrm{O}} V$. Again, the obstacles are independently placed at random locations before the start of the simulation, irrespective of possible overlap with other obstacles. It turns out that even with this rather restrictive choice for the number of obstacles (meaning not to allow Poissonian fluctuations) the number of obstacle realizations required for accurate disorder averages is still high (in the order of a few thousand). This is shown in figure 7.1, where the relative uncertainty in $\left[\chi_{2}\right]$ at two different fugacities is shown, where $\chi_{2}$ is the susceptibility that is defined in equation 3.10 . 

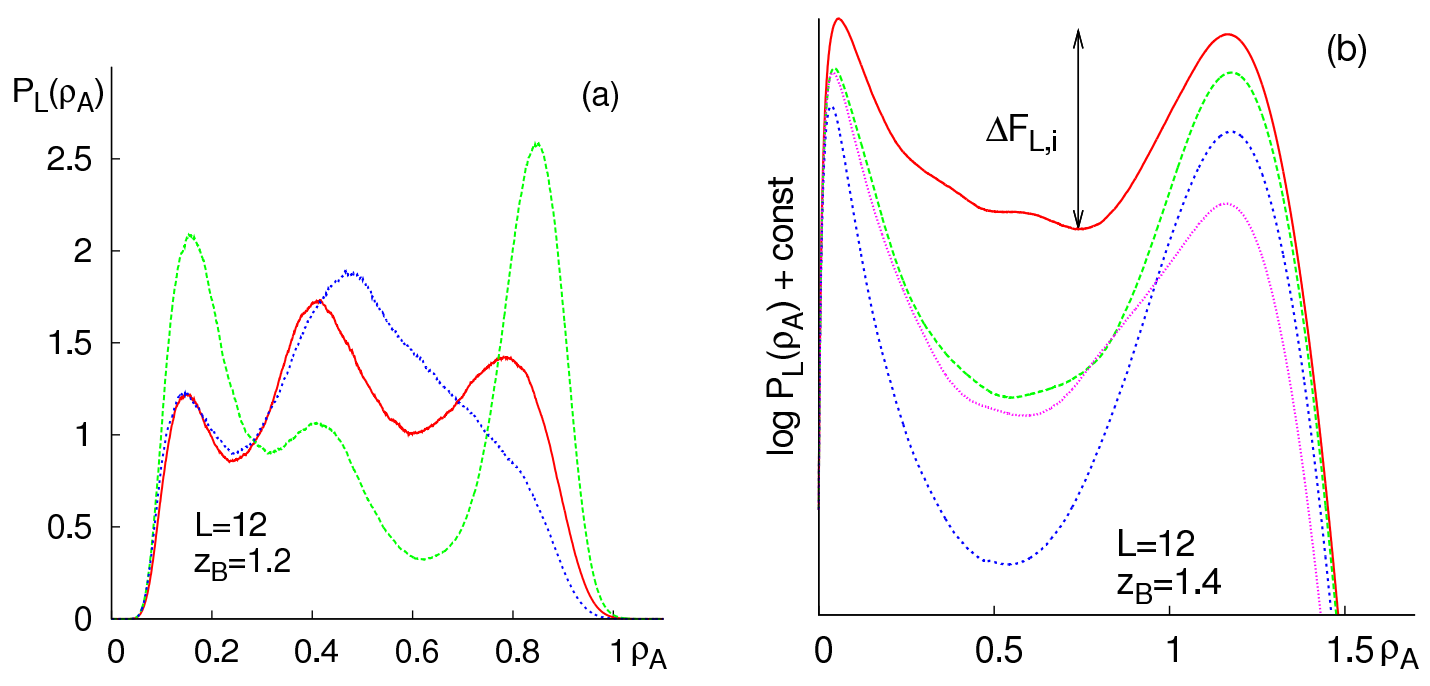

Figure 7.2: Around the pseudocritical $z_{\mathrm{B}}$ the OPDs for three-dimensional WR models with asymmetric obstacles can assume exotic shapes exhibiting an additional peak at intermediate density that cannot be attributed to a supercritical state. As shown in figure (a) this peak can either replace one of the two expected peaks (liquid and gas) or even appear as a third peak. Around the critical fugacity of the $L \rightarrow \infty$ system the OPDs are bimodal with identifiable liquid and gas peaks. This allows extracting a sensible free energy barriers $\Delta F_{L, i}$, as indicated by the vertical arrow. The curves on the right plot are normalized to $\log P_{L, i}(0)=0$ for better visualization.

\subsubsection{Tuning to Phase Equilibrium}

The calculation of disorder-averaged observables is performed exactly as in the case of the symmetric obstacles discussed in the previous chapter: For each $z_{\mathrm{B}}$ a suitable $z_{\mathrm{A}}$ is selected according to the locus $l_{\mathrm{MS}}$ (equation 3.22). From the OPD at these fugacities, the sought-for observables are then evaluated. But contrary to the case of symmetric obstacles the choice of $z_{\mathrm{A}}$ according to some coexistence locus criterion is not merely a correction to inaccuracies in the Monte-Carlo sampling: While the ensemble containing all possible obstacle configurations preserves the $\mathrm{A} \leftrightarrow \mathrm{B}$ symmetry of the WR model, a single obstacle configuration does not. Choosing an individual $z_{\mathrm{A}, i}$ for each configuration $i$ reflects that individual configurations do not have an $\mathrm{A} \leftrightarrow \mathrm{B}$ symmetry, and instead favor either particle species to a varying degree. This can be considered a finite-size effect and in the thermodynamic limit this variation around the disorder ensemble's symmetry line $z_{\mathrm{A}}=z_{\mathrm{B}}$ is expected to vanish ${ }^{26}$. 

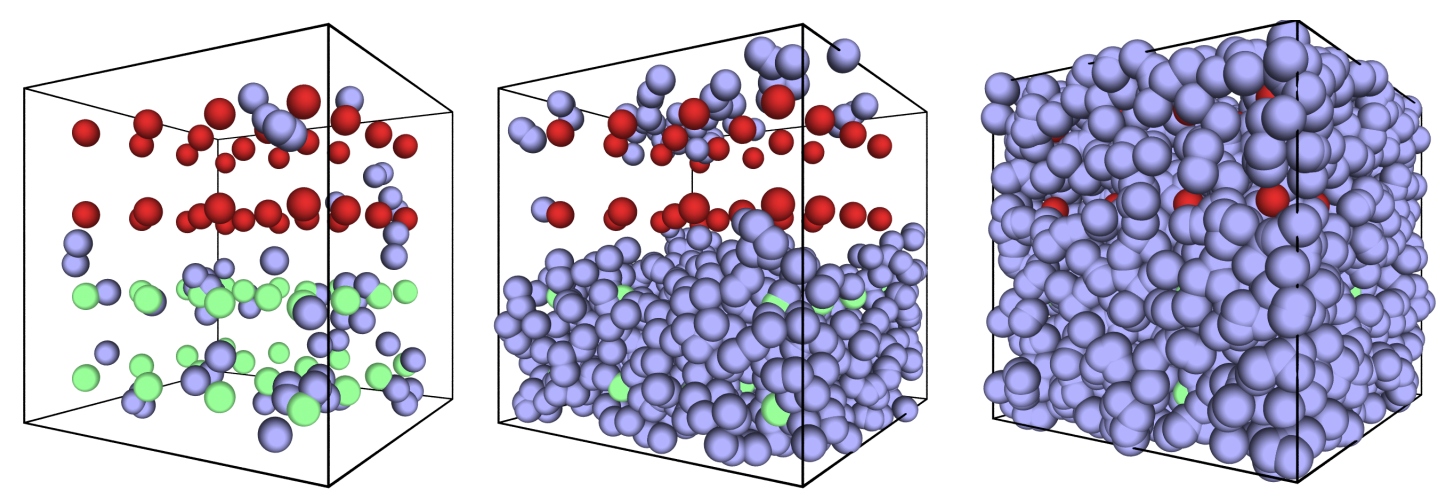

Figure 7.3: The obstacle configuration in which all of the A-affine obstacles (red) sit on a regular grid in one half of the volume and the B-affine obstacles (green) sit on a regular grid in the other half is an extreme example of a system in which three stable states exist even for high $z_{\mathrm{B}}$. These are the normal gas (left image) and liquid state (right image) for $z_{\mathrm{A}} \ll z_{\mathrm{B}}$ and $z_{\mathrm{A}} \gg z_{\mathrm{B}}$, respectively, and a state in which only the A-affine region is filled with A particles (center image) for $z_{\mathrm{A}} \approx z_{\mathrm{B}}$. Only the obstacles and the A particles (blue) are shown; the $\mathrm{B}$ particles are omitted for clarity.

\subsection{Pseudophases}

It turns out that at fugacities around the maximum of $\left[\chi_{2}\right]$ there are large variations between OPDs of different obstacle configurations. While in the case of the neutral obstacles all OPDs are qualitatively the same there are now strong deviations between shapes. In some cases extra peaks at intermediate densities appear alongside a liquid or a gas peak (see figure 7.2a).

The appearance of such peaks at intermediate densities can be explained by the existence of large regions with a preference for either of the particle species. If the obstacle configuration features such a cavity, a state in which the cavity is filled with its preferred particle species and the rest of the system is filled with the opposite species can become favorable. The unfavorable interface between the cavity and its surrounding is then compensated by both the cavity and the surrounding containing the particle species which they prefer. An extreme example of such an obstacle configuration is shown in figure 7.3, where all obstacles are arranged on a regular grid such that the obstacles with a preference for the A particles are in the lower half of the box and the obstacles preferring the B particles are in the upper half. As readily seen from the simulation snapshots, depending on $z_{\mathrm{A}}$ there are three distinct states for the system: the gas state, the liquid state, and the intermediate state where only the lower half of the box is filled with A particles.

The appearance of a third peak has been noted previously and has been interpreted as a possible signature of a first-order phase transition ${ }^{99}$ or an indication for the existence of an 

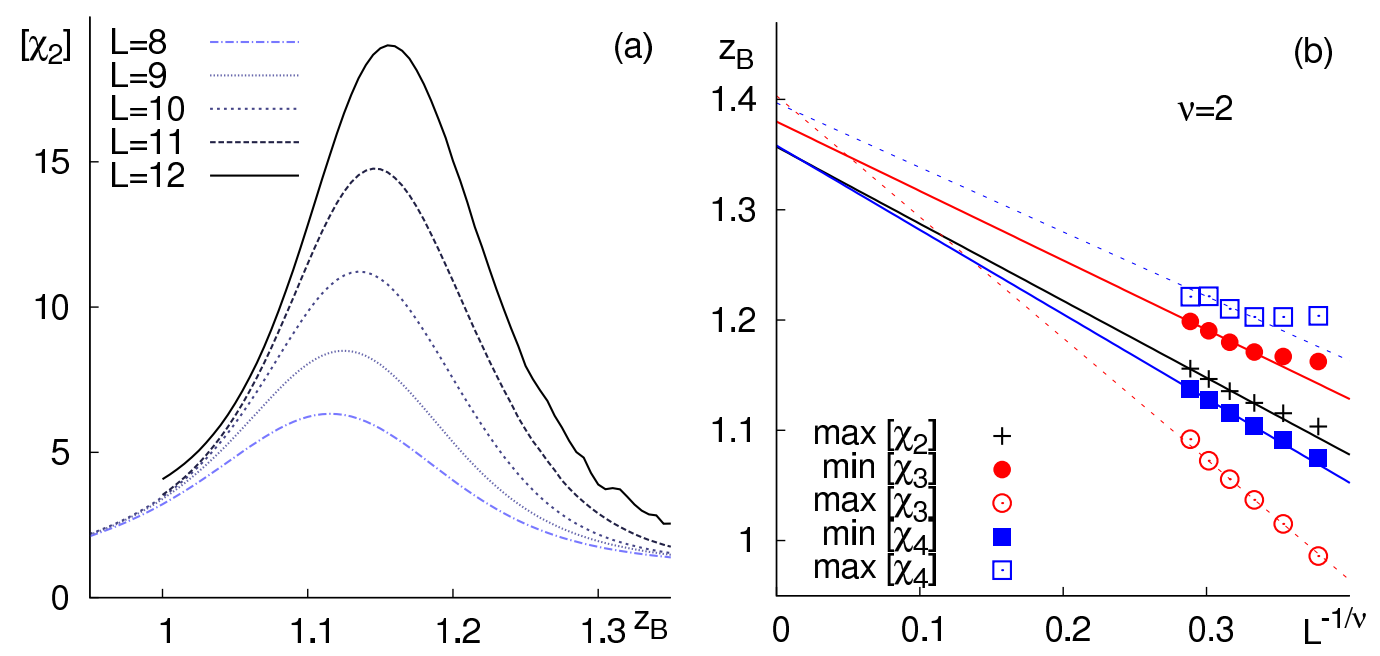

Figure 7.4: Finite-size scaling of the pseudocritical fugacities for a WR mixture with $\rho_{\mathrm{O}}=0.02$ obstacles of two species. As shown in figure (a) on the example of $\left[\chi_{2}\right]$, the disorder averages of the susceptibilities show the expected qualitative behavior despite the occurrence of exotic order parameter distributions like those shown in figure 7.2: A well-defined peak that systematically grows and shifts with increasing system size is seen. In figure (b) the attempt of an extrapolation towards a critical fugacity is shown. The predictive power of the extrapolation is restricted by the large distance of the critical fugacity from the pseudocritical fugacities, which makes the results sensitive to inaccuracies. Only an approximate interval for the critical fugacity and the correlation length exponent can be obtained.

additional phase ${ }^{27}$. The obstacle configuration shown in figure 7.3 can indeed exhibit three phases in the thermodynamic limit if scaled up appropriately ${ }^{100 ; 101 ; 102}$. Of course, since the disorder ensemble considered here is that of randomly placed obstacles an obstacle configuration as shown in figure 7.3 will effectively not exist in the thermodynamic limit, since the probability for such an ordered arrangement approaches zero as $L \rightarrow \infty$. At the critical point the occurrence of such exotic OPDs is expected to become a rare event ${ }^{24}$.

\subsection{Extrapolating Pseudocritical Fugacities}

Again, the extrapolation of pseudocritical fugacities towards the thermodynamic limit is expected to yield the critical fugacity for this model. As previously, the disorderaveraged susceptibilities $\left[\chi_{2}\right],\left[\chi_{3}\right]$, and $\left[\chi_{4}\right]$ show local extrema that can be attributed to pseudocritical points. However, the functionals $\chi_{2}, \chi_{3}, \chi_{4}$ were designed to work for OPDs featuring two (possibly overlapping) peaks and to be stable against the appearance of OPDs with a single peak (see chapter 3.1). For more exotic OPDs it is not exactly clear what these functional measure. However, as shown on the example of $\left[\chi_{2}\right]$ in figure 7.4(a) the disorder averages of the functionals qualitatively behave as expected, indicating 

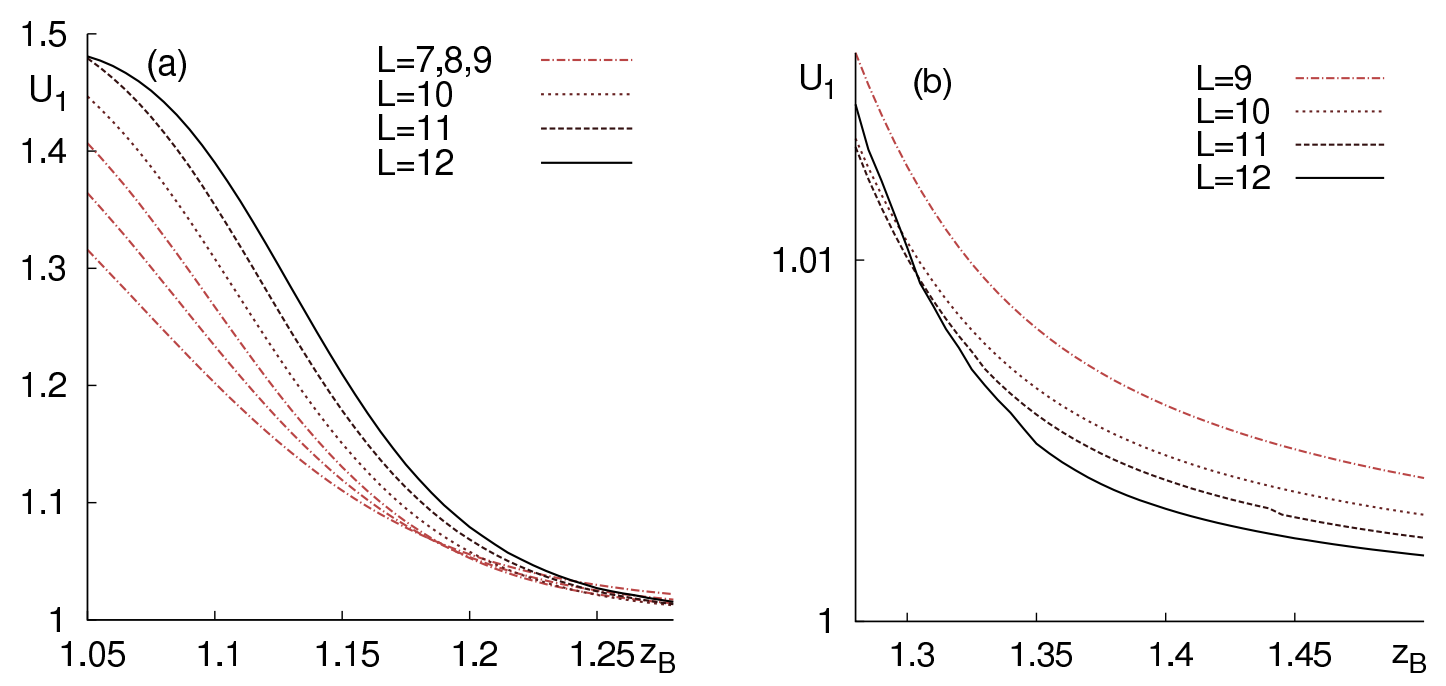

Figure 7.5: Values of the disorder-averaged cumulant $U_{1}$ (equation 6.3) around the pseudocritical fugacities (figure a) and the extrapolated region of the critical fugacity (figure b). As predicted by modified hyperscaling, no common intersection point that would indicate a critical point with normal hyperscaling is seen. The intersection of the curves for the three smallest system sizes in figure (a) is interpreted as a crossover effect from Ising universality ${ }^{24 ; 25}$.

that the functionals may still give an appropriate measure of the susceptibilities. As previously, the locations of the functionals' extrema are considered as the pseudocritical points.

Contrary to previous cases, all of the pseudocritical fugacities increase with the system size. This means that this time there is no interval in which the extrapolated critical point is guaranteed to lie, but that $z_{\mathrm{B}, \mathrm{cr}}$ can in principle assume any value above the largest pseudocritical fugacity. Related to this problem (and actually worsening it) is that the correlation length exponent $\nu$ is not well known for the RFIM ${ }^{7 ; 24}$. Lastly, the prospected critical fugacity is much larger than the pseudocritical fugacities. This results in the extrapolated critical fugacity to be very sensitive to errors in the peak locations. Considering that e.g. the value of $\left[\chi_{2}\right]$ is expected to be erroneous by $10 \%-30 \%$ (see figure 7.1), these are not expected to be determined very precisely. Indeed, the extrapolated lines in figure 7.4(b) do not agree on a single and unique value for the critical point, but (as shown) merely scale towards a common interval that is not too large. Results of the quality shown are obtained for correlation length exponents $\nu \in[1.3 ; 2.4]$, and result in critical fugacities in the range of $z_{\mathrm{B}, \mathrm{cr}} \in[1.3 ; 1.42]$. 


\subsection{Modified Hyperscaling}

The other candidate method for the location of the critical fugacity that has been introduced in section 3.2 is the cumulant method, that does not rely on extrapolation of data and may thus seem like a promising candidate method to obtain a more precise localization of the critical point. However, for RFIM universality this method does not work: If one assumes that there is a critical point in the 3D RFIM, then that point must show qualitative differences to the behavior of the pure Ising model. Particularly, hyperscaling (equation 3.18) does not hold and is replaced with a modified hyperscaling relation ${ }^{103}$

$$
2-\alpha=2 \beta+\gamma=\nu(d-\theta)
$$

The newly-appearing critical exponent $\theta$ is the violation of hyperscaling exponent, for which it is assumed that $\theta \approx 1.5^{24}$. As a direct consequence of hyperscaling violation no common intersection point in the cumulant curves for different system sizes at the critical point is expected, anymore. As shown in figure 7.5 there is indeed no common intersection point, neither around the pseudocritical fugacities (figure 7.5a) nor at the prospected region of the critical fugacity (figure $7.5 \mathrm{~b}$ ).

The $U_{1}$ cumulant can be considered as a measure for the ratio of the thermal fluctuations in the order parameter $|m|$ divided by the thermal average. In the presence of quenched disorder a similar quantity can be defined, which is the disconnected cumulant, defined as the fluctuations of the order parameter $\langle|m|\rangle$ between different obstacle configurations divided by the disorder average $[\langle|m|\rangle]$ :

$$
U_{1, \mathrm{dis}}=\frac{\left[\langle|m|\rangle^{2}\right]}{[\langle|m|\rangle]^{2}}
$$

It is expected that due to modified hyperscaling the disconnected cumulant curves for different system sizes intersect at the critical point the same way that the normal Binder cumulant does in the pure model ${ }^{24}$. However, this method does not work well in this case. Instead, a new method based on the scaling of the free energy barrier with system size has been employed ${ }^{24}$.

\subsubsection{The Free Energy Barrier}

As shown in figure $7.2(\mathrm{~b})$ at $z_{\mathrm{B}}=1.4$ (the prospected vicinity of the critical point) the order parameter distributions exhibit two well-separated peaks. This allows to extract a free energy barrier $\Delta F_{i}$ for each obstacle configuration $i$ in the same way as for the pure model (section 5.1.1). In the region where an $\mathrm{A}$ and a $\mathrm{B}$ phase coexist this barrier again corresponds (for sufficiently large systems) to the contribution of the interface between 

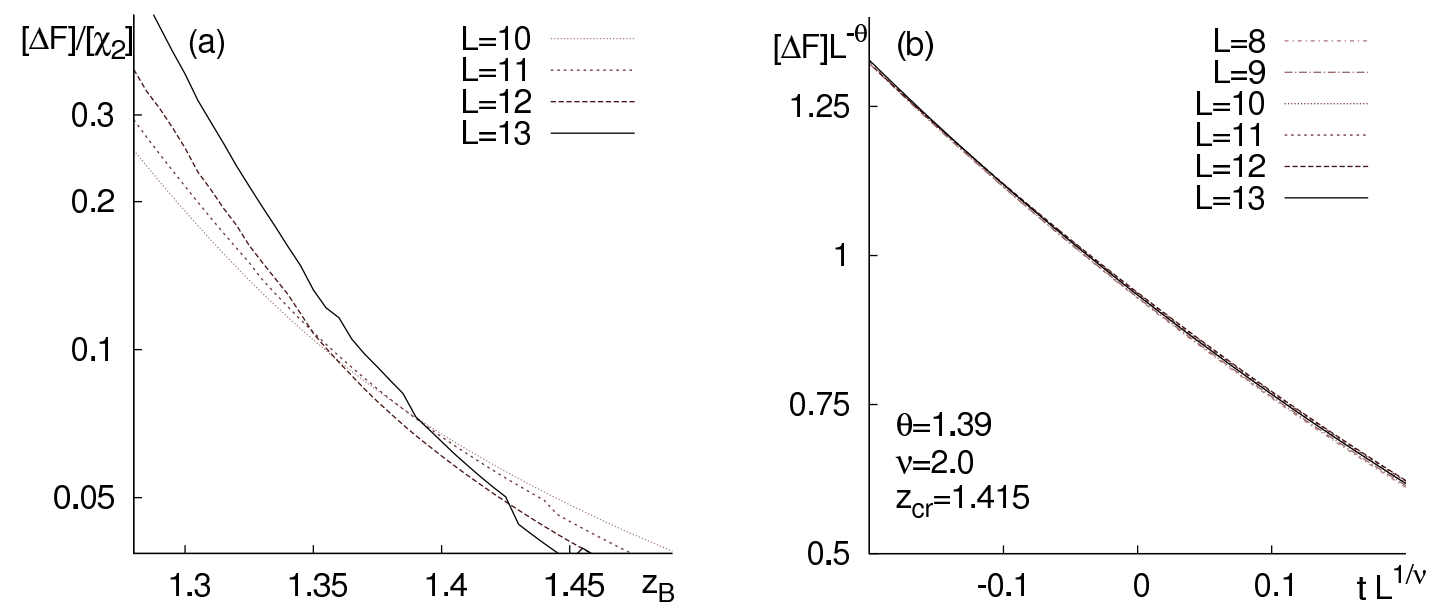

Figure 7.6: Finite-size scaling for a WR system (with $\rho_{\mathrm{O}}=0.02 \mathrm{~A}$-affine and B-affine obstacles) based on the scaling relation (7.3). Figure (a) shows the ratios $[\Delta F] /\left[\chi_{2}\right]$ for different system sizes $L$ which (assuming $\theta=\gamma / \nu$ ) intersect at the critical fugacity. Due to limited accuracy of the data the curves do not intersect at a single point, but a candidate region for the location of the critical fugacity is seen that is compatible with that found in figure 7.4(b). Figure (b) demonstrates the scaling of the free energy barrier as described in the text. Visually, an excellent agreement of the curves for different system sizes is achieved (but see also figure 7.7).

the phases. So for $z_{\mathrm{B}}>z_{\mathrm{B}, \mathrm{cr}}$ one expects $[\Delta F] \sim L^{2}$. Below the critical fugacity, $[\Delta F]$ is assumed to decrease with system size (if it can be extracted in the first place), owing to the fact that the OPD of a sufficiently large system in the supercritical phase is just a Gaussian distribution.

Contrary to the case of the pure WR model (or Ising model) precisely at an RFIM critical point $[\Delta F]$ does not become independent of system size. This can be seen from the scaling laws as the critical fugacity is approached from $z_{\mathrm{B}}>z_{\mathrm{B}, \mathrm{cr}}$. For two coexisting phases, the free energy barrier is proportional to the size of the interface $L^{d-1}$ and to the interface tension $\sigma$ (see section 5.1.1). By the Widom scaling relation ${ }^{104} \sigma \sim t^{2-\alpha-\nu}$, where $t$ is the reduced temperature. By using the modified hyperscaling relation (7.1) and the cut-off scale $\xi \sim L$ for the correlation length one finds that ${ }^{24 ; 100}$

$$
\begin{aligned}
{[\Delta F] \underset{(7.1)}{\sim} \sigma L^{d-1} \sim t^{2-\alpha-\nu} L^{d-1} \stackrel{(3.13)}{\sim} \xi^{(\alpha+\nu-2) / \nu} L^{d-1} \stackrel{\xi \sim L}{\sim} L^{(\alpha+\nu d-2) / \nu} } & \\
& =
\end{aligned}
$$

Note that the same calculation can be performed for the pure model where the free energy barrier at the critical point becomes independent of system size (as $\theta=0$, there).

For the RFIM it is assumed that the violation of hyperscaling exponent is not an independent critical exponent but related to the other critical exponents by $\theta=\gamma / \nu^{103}$. In conjunction with the scaling law (7.3) this implies that at the critical point the ratio 
$[\Delta F] /\left[\chi_{2}\right]$ should become independent of the system size ${ }^{24}$. Consequently, when this ratio is plotted over $z_{\mathrm{B}}$ the curves for different system sizes should intersect at the critical fugacity. As seen in figure 7.6(a) the curves indeed cross in some intersection region, indicating the location of the critical point in this interval. This intersection interval $z_{\mathrm{B}} \in[1.35 ; 1.45]$ is consistent with the critical fugacity estimates found via finite-size scaling of the extrema in $\left[\chi_{2}\right],\left[\chi_{3}\right]$ and $\left[\chi_{4}\right]$ found in section 7.3.

If around the critical point a scaling law like (3.16) also holds true for the free energy barrier, then

$$
[\Delta F]=L^{\theta} f\left(\left(z_{\mathrm{B}}-z_{\mathrm{B}, \mathrm{cr}}\right) L^{1 / \nu}\right)
$$

where $f$ is the universal scaling function. This means that when $[\Delta F] \cdot L^{-\theta}$ is plotted as a function of $z_{\mathrm{B}} L^{1 / \nu}$ for different system sizes, the different curves should fall on top of each other if the correct values for the critical exponents $\theta$ and $\nu$ and the correct critical fugacity $z_{\mathrm{B}, \mathrm{cr}}$ are used. Conversely, by varying these three parameters until a good collapse of the curves is found $\theta, \nu$, and $z_{\mathrm{B}, \mathrm{cr}}$ can be determined. In figure 7.6(b) a collapse of the curves for different system sizes is shown. The values $\nu=2, z_{\mathrm{B}, \mathrm{cr}}=1.41$ are in good agreement with the results previously found; the value of the critical exponent $\theta=1.39$ is in reasonable agreement with the expectation that $\theta \approx 1.5$.

It is tempting to consider the collapse of the curves in 7.6(b) as an indication that the critical exponents and the critical fugacity are determined very accurately. This is a dangerous assumption because three independent fit parameters $\left(\theta, \nu\right.$, and $\left.z_{\mathrm{B}, \mathrm{cr}}\right)$ provide many degrees of freedom that can be used to improve the quality of the collapse. More importantly, it is not clear that what visually looks like a "good collapse" of the curves actually corresponds to a good collapse on a reasonable physical scale. To tackle this issue, an measure of the collapse quality has been developed that not only allows to find a set of optimal parameters, but also quantifies the consistency of any set of parameters with the data and therefore allows to estimate the statistical accuracy to which $z_{\mathrm{B}, \mathrm{cr}}, \nu$, and $\theta$ are measured.

\subsubsection{Likelihood of Critical Exponents}

To quantify the quality of a collapse, the deviations between the curves are compared to the uncertainties that are expected due to the necessarily finite number of $N$ obstacle configurations being considered. For any given values for $\theta, \nu, z_{\mathrm{B}, \mathrm{cr}}$ and for each $\tau:=t L^{1 / \nu}$ the values $y_{L}:=[\Delta F] L^{-\theta}$ for different system sizes $L$ scatter around their average with a variance of $\sigma^{2}$. This variance is compared to the statistical uncertainty expected due to the finite number of $N$ obstacle configurations. The square of the statistical uncertainty of $[\Delta F]$ is

$$
u_{L}^{2}=\frac{1}{\sqrt{N(N-1)}} \sum_{i=1}^{N}\left(\Delta F_{i}-[\Delta F]\right)^{2} .
$$




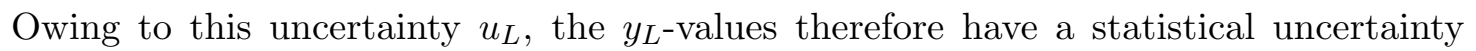
$\delta y_{L}:=u_{L} L^{-\theta}$.

Now, for any $\tau$ let $\delta y$ be the average of the $\delta y_{L}$, averaged over the different system sizes. The ratio $\sigma / \delta y$, then is a measure of the scatter between the different curves compared to the statistical uncertainty - for that particular value of $\tau$. Since the observable of interest is the quality of the collapse in some region around the critical point this ratio is averaged over some interval $[-\Delta \tau ;+\Delta \tau]$ around the critical point (which is at $\tau=0$ ). This finally gives rise to the likelihood-variable quantifying the quality of the collapse:

$$
R\left(\theta, \nu, z_{\mathrm{B}, \mathrm{cr}}\right):=\frac{1}{2 \Delta \tau} \int_{-\Delta \tau}^{\Delta \tau} \frac{\sigma(\tau)}{\delta y(\tau)} \mathrm{d} \tau
$$

Values of $R<1$ imply that the fluctuations between the curves are smaller than random deviations, and can therefore be considered as fully consistent with a perfect collapse. Values $R>1$ are interpreted as increasingly less consistent. The integration range has been chosen as $\Delta \tau=0.2 \cdot 10^{-1 / \nu}$, meaning that e.g. for the $L=10$ system the integration range is $|t| \leq 0.2$. The choice is a compromise between the proximity to the critical point (so that scaling still applies) and a non-zero integration range (to avoid mis-interpreting an intersection at a single point as a collapse of the curves). By numerically minimizing $R$, the best-fitting parameters have been found to $\mathrm{be}^{100} z_{\mathrm{B}, \mathrm{cr}}=1.42, \theta=1.43$, and $\nu=2.31$.

\section{Interpretation of the Likelihood Variable}

The likelihood variable $R$ is a measure of compatibility of the data with the test hypothesis that for the given parameters the curves $y_{L}(\tau)$ for different system sizes become identical around $\tau=0$. Since it has been constructed as the ration of the curves' scatter to a standard deviation of the statistical fluctuations the value of $R$ can be interpreted as measuring by how many statistical standard deviations the result differs from the expected result if the hypothesis of scaling with the respective parameters were valid. This allows for an analysis that is much more interesting than obtaining a single point of best collapse, namely to define some criterion for accepting the hypothesis of scaling with the given parameters (e.g. $R \leq 2$ ), and then to identify the regions in the parameter space that according to this criterion are possible values for $\theta, \nu$, and $z_{\mathrm{B}, \mathrm{cr}}$.

In figure 7.7 such candidate regions for the critical exponents are shown, where for each point in the $(\theta, \nu)$ plane $R$ has been minimized with respect to $z_{\mathrm{B}, \mathrm{cr}}$. The left plot shows the resulting $R$ values for the $\rho_{\mathrm{O}}=0.02 \mathrm{WR}$ mixture with two species of asymmetric obstacles discussed throughout this chapter, and with the system sizes $L=7,8, \ldots, 12$ being taken into account. The right plot shows the same analysis, but for free-energy data extracted from an actual random-field Ising model with uncorrelated Gaussiandistributed random external fields acting on each lattice site. The $\Delta F_{i}$ data for the RFIM 

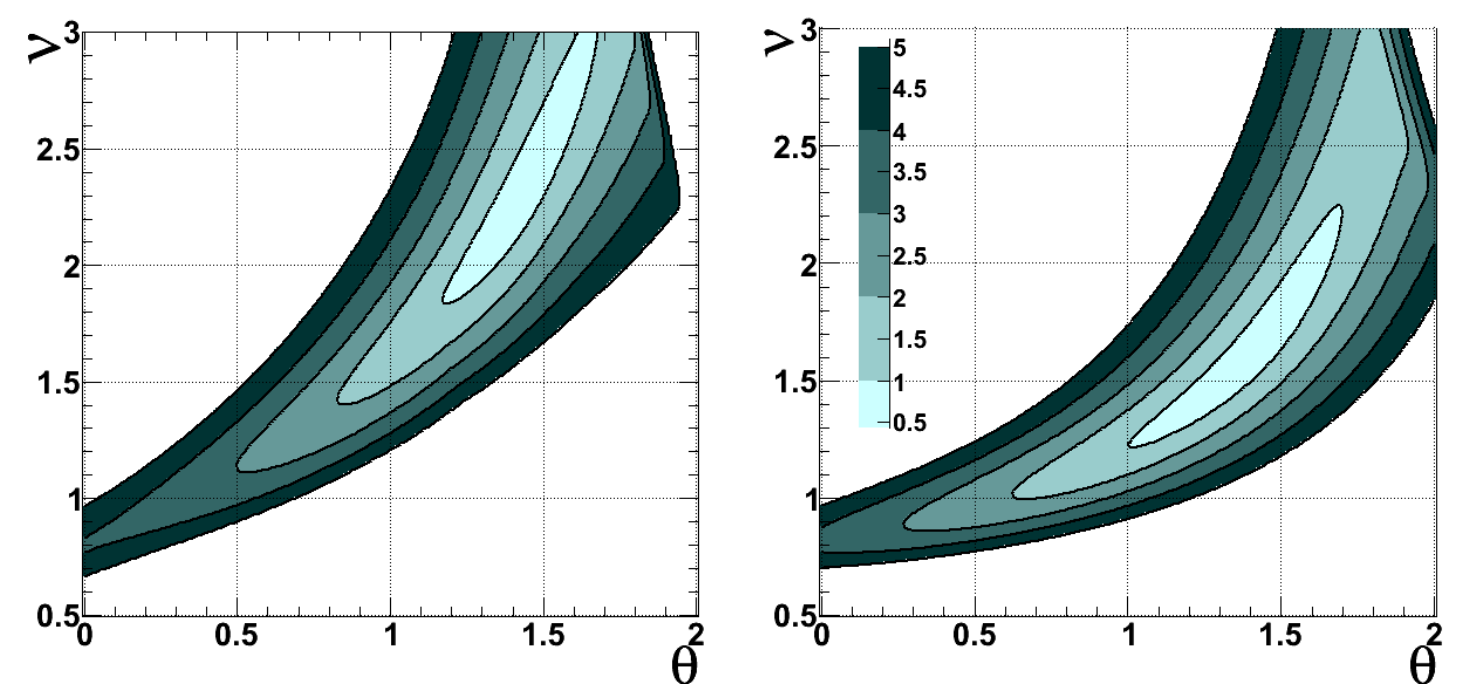

Figure 7.7: Likelihoods of the critical exponents $\theta$ and $\nu$ for the 3D WR model with $\rho_{\mathrm{O}}=0.02 \mathrm{~A}$ affine and B-affine obstacles (left plot) and the 3D RFIM on a cubic lattice ${ }^{24 ; 100}$ (right plot). The plots show the color-coded $R$ values for the best-fitting critical fugacity (or critical temperature) at the respective point in the $(\theta, \nu)$ plane; the scale bar in the right plot also applies to the left plot. Both plots favor $\theta \approx 1.5$ as expected for the RFIM, and exclude $\theta=0$ and $\theta=d-1=2$ by at least $R>3$.

were provided by Richard Vink ${ }^{24 ; 100}$; the systems taken into account are cubes with $L=8,10, \ldots, 16$ spins in each direction and periodic boundary conditions.

In both models an exact determination of the critical exponents is not possible, but consistence with the expected RFIM criticality can be shown. Both data sets prefer the expected $\theta \approx 1.5$, and the two "competing" values $\theta=0$ for a conventional critical point with hyperscaling and $\theta=d-1=2$ for a first order phase transition both are effectively excluded as they lie in the $R>3$ region. The possible regions for the correlation length exponent $\nu$ exclude $\nu \approx 0.63$ of the pure Ising universality. For the WR model, the best-fitting critical fugacities in the region $R \leq 1, \theta \leq 1.5$ are $z_{\mathrm{B}, \mathrm{cr}} \in[1.31 ; 1.48]$, which is consistent with the results obtained from extrapolating the pseudocritical fugacities and from the intersection region found in figure 7.6(a). The de-facto exclusion of $\theta=0$ and $\theta=2$ for the WR mixture with random asymmetric obstacles shows the existence of a point in the phase diagram that is neither an Ising critical point nor a first-order phase transition. The same is seen for the RFIM data, which confirms the assumption that both models are in the same universality class. 


\subsection{Conclusion: The Universality Class of 3D Mixtures and Fluids in the presence of Quenched Disorder}

In this chapter the three-dimensional Widom-Rowlinson mixture with randomly located static obstacles that have an asymmetric coupling to the two species of the mixture has been investigated. By means of disorder averages over a large set of $N \approx 5000$ obstacle configurations for each system size the existence of a critical fugacity much above that of the pure model was found. The scaling behavior of the free energy barrier $\Delta F$ has been investigated and a criterion to quantify the accuracy of estimates for the violation of hyperscaling exponent $\theta$ has been developed and applied. By comparison with simulation data of the random-field Ising model it has been confirmed that the universality class of the mixture with asymmetric obstacles is that of the RFIM. This is consistent with earlier findings for another non-additive hard sphere mixture with static obstacles, the Asakura-Oosawa mixture ${ }^{26}$. While a precise measurement of the violation of hyperscaling exponent $\theta$ has not been possible, a conventional critical point obeying hyperscaling and a first order phase transition are excluded by the results. The $R \leq 1$ candidate region for $\theta$ found in both models, the WR mixture and the actual random-field Ising model, is in good agreement with newer and more precise results ${ }^{9}$.

In all of the above an equal density of A-affine and B-affine obstacles has been used. This choice has been made for convenience only, as it simplifies the numerical analysis because $\mathrm{A} \leftrightarrow \mathrm{B}$ symmetry is restored in the average over all disorder configurations. Just as an Ising model with random fields not drawn from a distribution with an average of zero still constitutes an RFIM model (since the shift from an average of zero is simply compensated by a non-zero homogeneous external field) an unequal number of both obstacle species should not change the universality class of the model, but merely shift the phase transition away from $z_{\mathrm{A}}=z_{\mathrm{B}}$. A particularly interesting choice of obstacles in this respect is using obstacles that have a hard-core repulsion with the A particles but no interaction with the B particles. In this case, the mapping of the WR mixture on a fluid model can still be performed, and maps on a fluid with obstacles that have a hard-core repulsion with the fluid particles. The results of this chapter can therefore also be considered a confirmation of the hypothesis by de Gennes ${ }^{13}$ that fluids in the presence of random obstacles should lie in the universality class of the random-field Ising model. 


\section{D Fluids and Mixtures with Asymmetric Obstacles}

In the previous chapter it has been argued that fluids in the presence of randomlydistributed non-neutral obstacles belong to the universality class of the random-field Ising model (RFIM). In $d=3$ dimensions this implies the existence of a liquid $\leftrightarrow$ gas transition that ends in a hyperscaling violating critical point. The case of fluids with quenched disorder in $d=2$ dimensions is radically different. It is known that for sufficiently weak fields even at zero temperature a spontaneous magnetization is absent, provided the proper homogeneous external magnetic field is applied ${ }^{97 ; 98}$. Furthermore, a first-order phase transition between phases of different magnetizations cannot exist, there ${ }^{10}$. Consequently, if a fluid with static obstacles indeed exhibits RFIM universality then no notion of liquid and gas, and hence no transition between these two phases should exist.

A famous argument for the elimination of long-range order in the 2D RFIM is the Imry-Ma argument ${ }^{105}$. Consider an RFIM system with magnetic fields acting on each lattice site such that the average of the random magnetic field is zero ${ }^{\mathrm{a}}$. Now, consider an ordered domain of spins characterized by a length scale $l$. The formation of such a domain is associated with an interface free energy cost that scales $\sim l^{d-1}$. The average over the random fields in the domain is zero, but exhibits Poissonian fluctuations. Thus, on average the formation of the domain of appropriate spin direction is associated with a free energy gain from aligning according to the random field that scales $\sim l^{d / 2}$. From the scaling of these two terms one sees that for $d \leq 2$ the formation of large domains (and thus the absence of a long-range order) is feasible, whereas for $d>2$ the interface contribution inhibits the formation of large domains.

A variant of the Imry-Ma argument (with the obstacles being interpreted as the random field) should hold for the Widom-Rowlinson mixture. In this chapter, the hypothesis of the absence of a liquid $\leftrightarrow$ gas transition for two dimensional fluids in the presence of quenched disorder is tested. In the mixture picture it is shown that at non-zero temperatures macroscopic demixing is replaced by the formation of small domains whose shapes are defined by the random obstacles. Finally, a possible real-world system where the findings may be of relevance, eukaryotic cells, is briefly discussed. Results from molecular dynamics

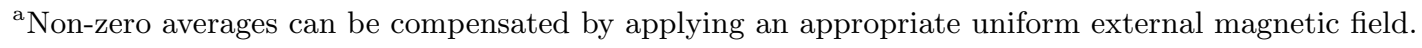


simulations that indicate the possible applicability of the WR model's results to the much more complicated cellular systems are presented.

\subsection{Choice of Quenched Disorder}

Two cases of random obstacles with a preferred affinity to one of the phases have been investigated, which are the maximally asymmetric obstacles and the semidiluted obstacles. The maximally asymmetric obstacles have a hard-core interaction with the A particles at a center-to-center distance of one, and do not interact with the B particles at all. This choice of obstacles is particularly interesting because it preserves the mapping of the WR mixture on a fluid model (see chapter 2.1.2). Results for the WR mixture with this choice of obstacles therefore directly imply results for a fluid model with random de-wetting obstacles. The semidiluted obstacles have a hard-core interaction with the A particles at a center-to-center distance of one, and a hard-core interaction with the $\mathrm{B}$ particles at a smaller center-to-center distance (0.75). Such obstacles destroy the mapping of the mixture on a fluid model, but are interesting in the sense that their interaction contains an aspect that should result in RFIM universality (the asymmetric coupling to the mixture species) and at the same time a dilution component that might point towards site-diluted Ising universality (the exclusion of volume for both species of the mixture). Such an additional dilution component is likely to be present also in real mixtures with quenched obstacles, such as a plasma membrane of different lipids and immobile proteins (see section 8.4). As will be shown, this additional dilution component does not destroy the RFIM universality of the mixture. This provides additional evidence that mixtures and fluids with any amount of asymmetric coupling of the obstacles behave as a random-field Ising model.

As in the case of the symmetric obstacles in chapter 6 , for each obstacle configuration representing a macroscopic obstacle density $\rho_{\mathrm{O}}$, the actual number of obstacles has been chosen at random from a Poissonian distribution with average $\rho_{\mathrm{O}} V$, and the obstacles have again been put into the system at random locations independently. For the simulation of the mixture, successive umbrella sampling with the single particle moves defined in section 4.2 has been employed — with the additional rule that MC steps that would lead to a forbidden overlap of a mixture particle and an obstacle are rejected. The pure model's exact result for the first window of successive umbrella sampling (equation 4.14) has been used as the sampling weight in the first window.

\subsection{Elimination of the Liquid $\leftrightarrow$ Gas Transition}

In figure 8.1 the order parameter distributions for eight randomly-selected configurations of semidiluted obstacles are shown for different fugacities. With the exception of the 

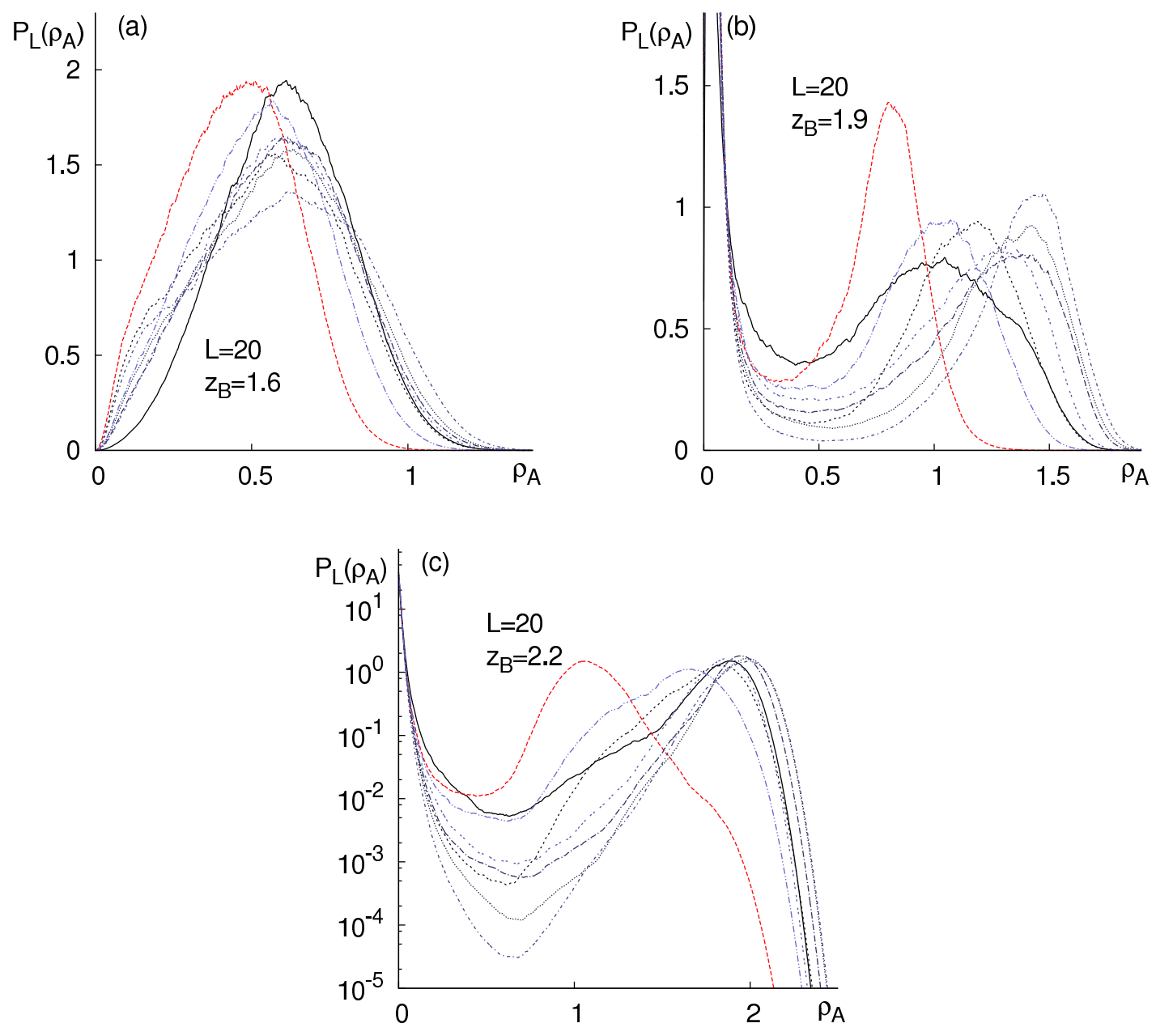

Figure 8.1: Randomly selected order parameter distributions for the two-dimensional $L=20$ WR mixture with $\rho_{\mathrm{O}}=0.04$ semidiluted obstacles for different fugacities. Same colors between different graphs correspond to the same obstacle configuration. The curves indicate the existence of a supercritical pseudophase at $z_{\mathrm{B}}=1.6$ (figure a) and the coexistence of a liquid and a gas pseudophase at $z_{\mathrm{B}}=2.2$ (indicated in figure (c) by the presence of a peak at high and low $\rho_{\mathrm{A}}$, respectively - note the logarithmic scale on this plot). At intermediate fugacities (figure b), overlapping peaks similar to the OPD around a critical point are observed. The OPD colored in red differs from the other OPDs in that it is not a liquid pseudophase that coexists with the gas pseudophase, but a pseudophase with only about half the A density of a liquid phase (see figure 8.2). Since it will be shown that in the thermodynamic limit coexistence of any two phases is absent, the actual nature of the coexisting pseudophases is not explicitly investigated. 

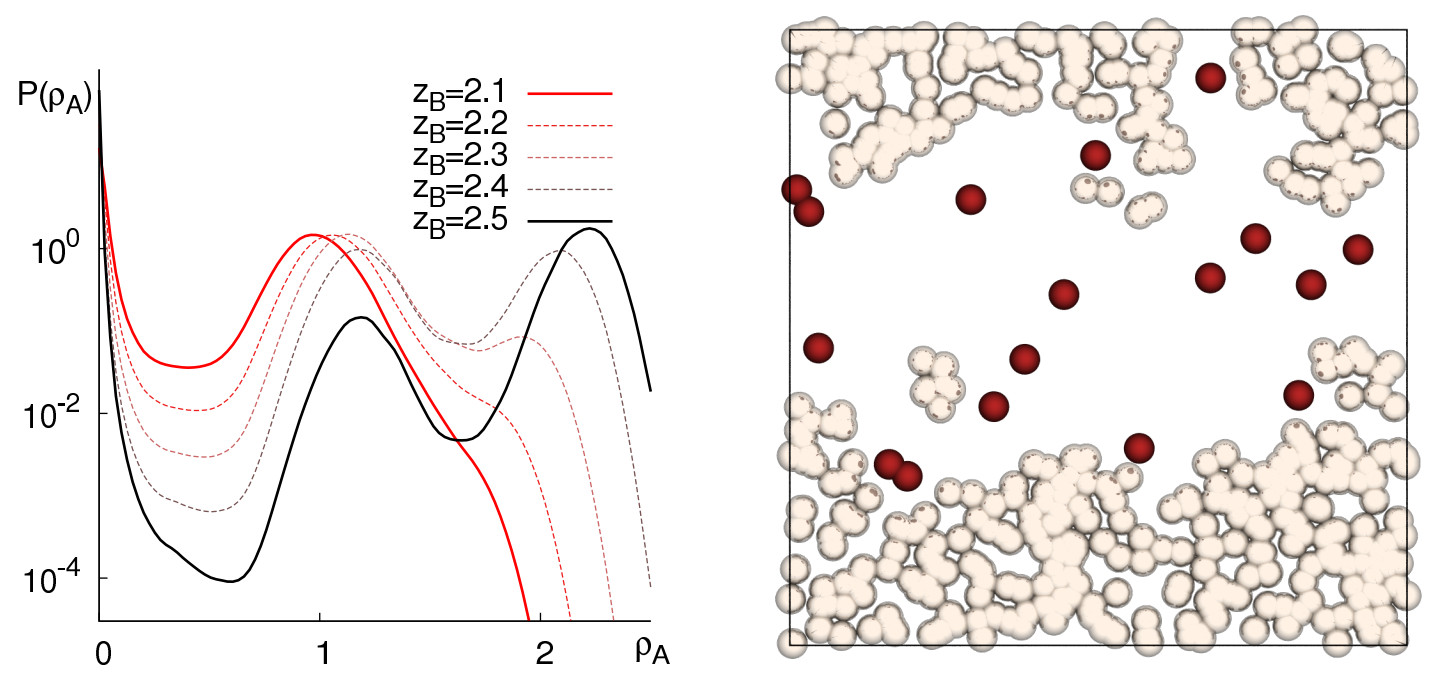

Figure 8.2: Detailed investigation of the obstacle configuration that lead to the order parameter distributions colored in red in figure 8.1. In the left plot, the OPDs for different $z_{\mathrm{B}}$ are shown (with $z_{\mathrm{A}}$ chosen according to $l_{\mathrm{MS}}$ of equation 3.22). For $z_{\mathrm{B}}=2.1,2.2,2.3$ coexistence between a gas and a "half-filled" pseudophase is seen, but at higher fugacities the half-filled pseudophase becomes suppressed and gas and liquid pseudophase exist. A typical state of the half-filled pseudophase is seen in the right image, where the A particles (pale) and the obstacles (red) are shown. The cause of this pseudophase is the random existence of a large cavity that is roughly half of the system (remember that periodic boundary conditions are used), and causes this half of the system to have a different affinity for the A particles than the other half (where the obstacles make the presence of A particles less favorable).

configuration whose OPD is colored in red, all curves are qualitatively consistent with the existence of a supercritical region at low $z_{\mathrm{B}}$, and the coexistence of an $\mathrm{A}$ and a $\mathrm{B}$ phase at high fugacities: At $z_{\mathrm{B}}=1.6$ the curves all show a single peak that can be attributed to a supercritical pseudophase. At $z_{\mathrm{B}}=2.2$, all OPDs with the exception of the red one show what could be attributed to the coexistence between a liquid and a gas pseudophase ${ }^{\mathrm{b}}$. At the intermediate fugacity $z_{\mathrm{B}}=1.9$ an overlap between two peaks is seen.

The only exception to this interpretation is the obstacle configuration colored in red. As shown in figure 8.2 this configuration is characterized by a large cavity, which in the sense of the Imry-Ma argument is a region that locally has less rejection for the A particles than the system on average. Note that by this definition a cavity is not required to be void of obstacles, and can also have an irregular shape. In this case, the large cavity causes the existence of a stable cavity-filling pseudophase (shown in figure 8.2) which

\footnotetext{
bStrictly speaking the case of semidiluted obstacles does not map on a fluid model, and "A pseudophase" and "B pseudophase" would be the formally correct terms. But since the qualitatively same results as with in the maximally asymmetric obstacles are seen, the less bulky terms "liquid" and "gas" are used throughout this section, instead.
} 

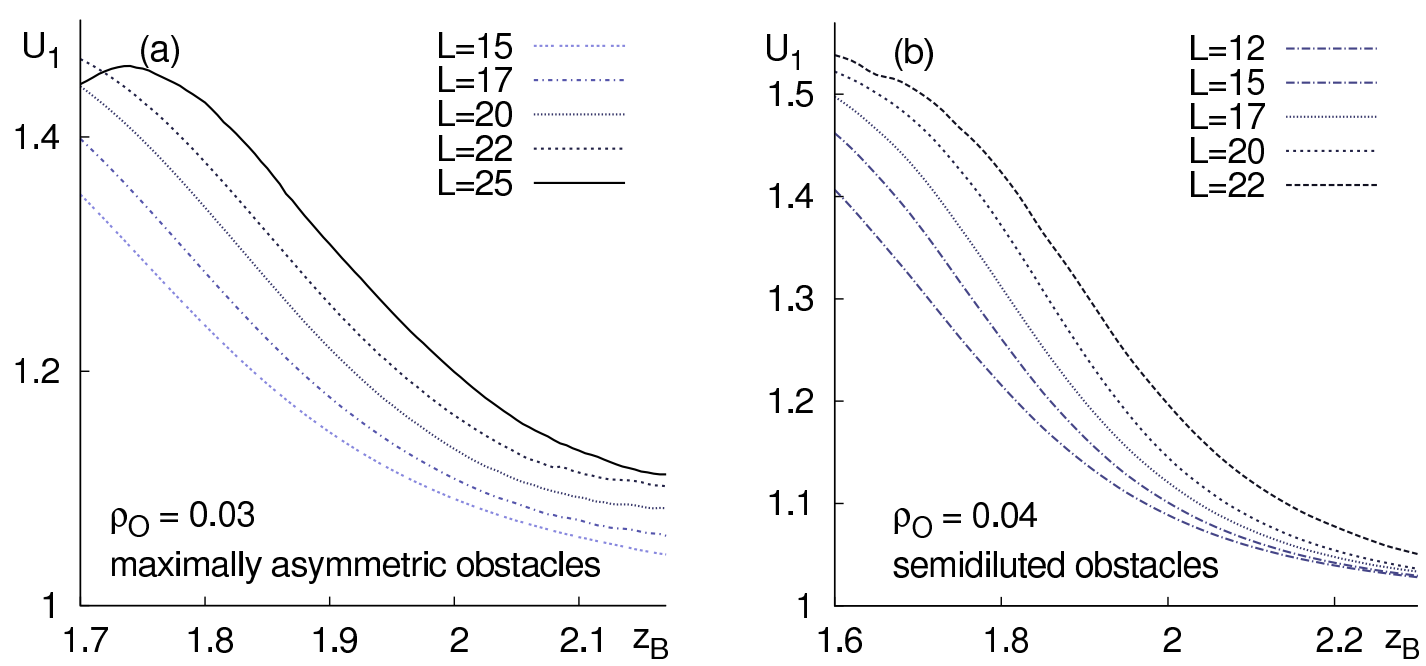

Figure 8.3: Results for the $U_{1}$ disorder averaged cumulant (equation 6.3) for the 2D WR model with the two different cases of asymmetric obstacles introduced in the text. All curves drop from values indicating a single pseudophase to $U_{1} \approx 1$, which indicates two coexisting pseudophases. No cumulant intersection is seen, which indicates the absence of a critical point with hyperscaling. Instead, the curves appear as if with increasing system size they are merely shifted towards higher $z_{\mathrm{B}}$ (see also figure 8.4). The apparent saturation of the curves in the left plot is an artifact of temperature reweighting reaching its limit (see section 4.3), as simulations were performed at the maximum slope of $U_{1}$. For the semidiluted obstacles simulation results from $z_{\mathrm{B}}=1.6,1.7, \ldots, 2.3$ were combined (section 4.3.2).

coexists with the gas pseudophase of figure 8.1(c). Such "cavity dominated" configurations appear more frequently in the two dimensional model than in three dimensions. This is expected by the Imry-Ma argument that explicitly claims that cavities become relevant in two dimensions. However, if $z_{\mathrm{B}}$ is increased to sufficiently high values the peak of the intermediate cavity-filling state in the OPD vanishes, and a coexistence between a liquid and a gas peak develops (see figure 8.2).

Considering that for this model RFIM universality and hence a lack of a notion of liquid and gas phase is expected, the consistent finding of order parameter distributions that can be attributed to the coexistence of a liquid and a gas pseudophase may be a surprise. The resolution of this apparent contradiction between theory and computer simulation is to consider finite-size effects. In figure 8.3 disorder averaged cumulants $U_{1}$ (equation 6.3) for different system sizes and both types of obstacles are shown. For each individual system size a decay of the cumulant value from supercriticality $\left(U_{1} \approx \pi / 2\right)$ to the coexistence of two pseudophases $\left(U_{1} \approx 1\right)$ is seen, but the transition region shifts towards larger $z_{\mathrm{B}}$ with increasing system size. As shown in figure 8.4(a), the same qualitative behavior holds true for the disorder averaged cumulant $\left[U_{1}\right]$ defined in equation 6.2. The data shown in figure 8.3 and 8.4 indicate that the minimum $z_{\mathrm{B}}$ at which coexistence between a liquid 

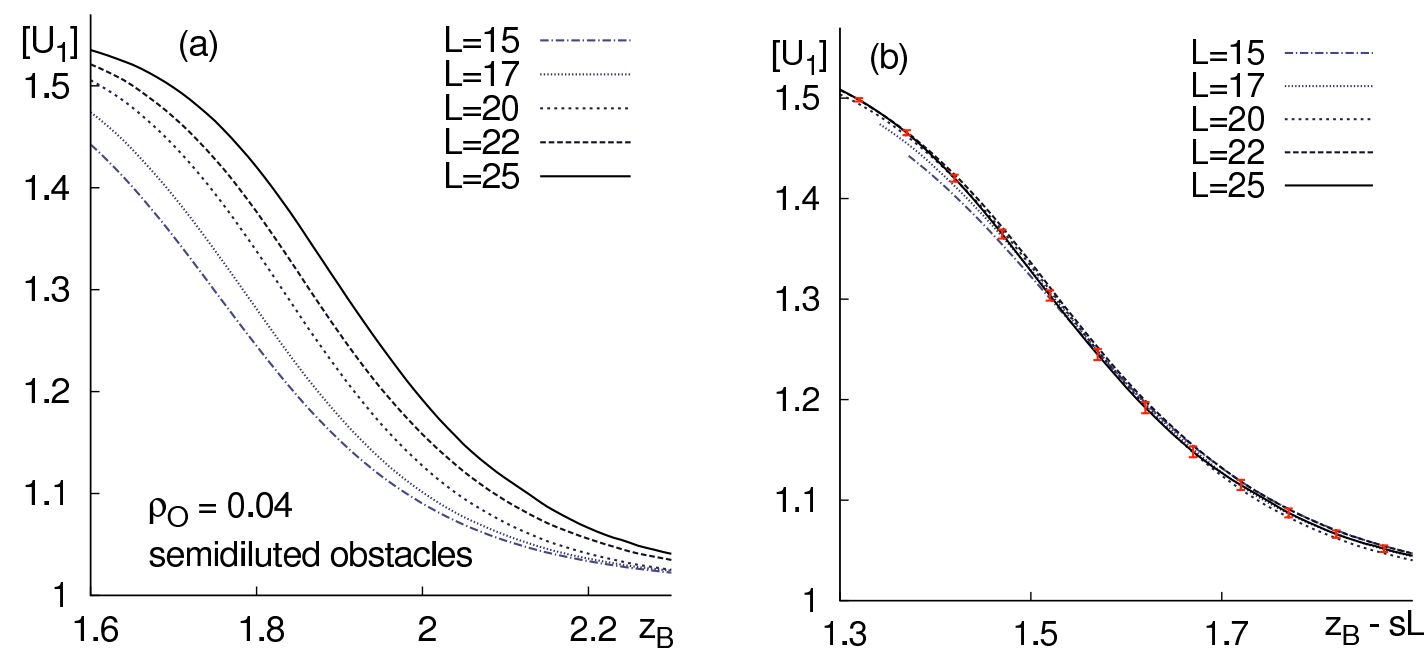

Figure 8.4: Results for the disorder averaged cumulant $\left[U_{1}\right]$ (equation 6.2) for the WR model with $\rho_{\mathrm{O}}=0.04$ semidiluted obstacles. Figure (a) shows $\left[U_{1}\right]$ as a function of $z_{\mathrm{B}}$, which qualitatively looks identical to the other definition of a disorder averaged cumulant shown in figure 8.3. In figure (b) the same data are displayed over $z_{\mathrm{B}}-s L$, where $s=0.0158(2)$ has been chosen such that the curves for different system sizes $L$ overlap. The red error bars shown are the uncertainties of the $L=25$ system due to the finite number of obstacle configurations being taken into account to compute $\left[U_{1}\right]$. The collapse of the curves seen is compatible with the statement that the fugacities at which a liquid and a gas pseudophase can coexist $\left(\left[U_{1}\right] \approx 1\right)$ diverge as $L \rightarrow \infty$. 

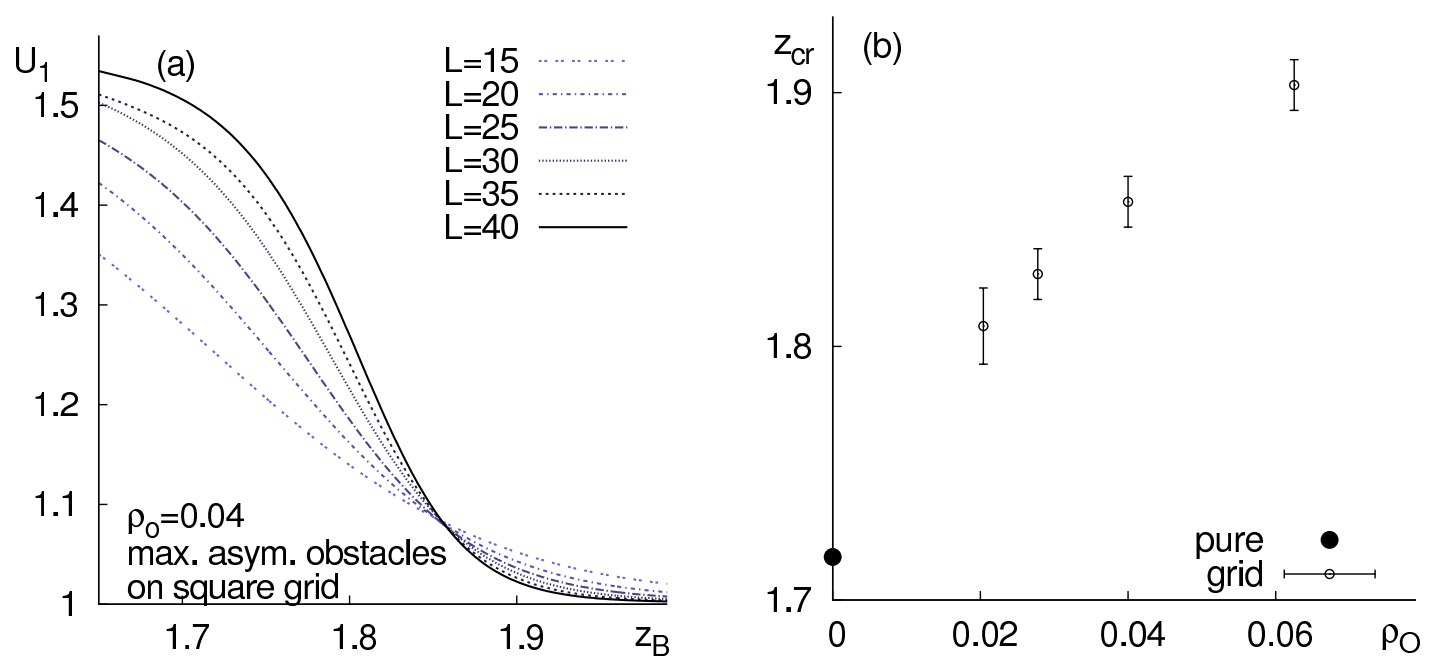

Figure 8.5: Results for maximally asymmetric obstacles arranged on a regular square grid. Figure (a) shows a cumulant intersection indicating a regular critical point with hyperscaling. In figure (b) the critical fugacities are shown as a function of $\rho_{\mathrm{O}}$. The critical fugacities are determined as a combined estimate from the intersections in the $U_{1}$ cumulant and the extrapolation of the locations of the $\chi_{2}$ maxima (error bars correspond to an "eye-gauged" combined uncertainty).

and a gas pseudophase can be observed approaches infinity as the system size approaches the thermodynamic limit. Thus, in the thermodynamic limit no coexisting gas and liquid exist for any finite $z_{\mathrm{B}}$. As shown in figure 8.4(b), the transition fugacities of the cumulant curves are indeed compatible with a linear increase with system size.

The above argument for the elimination of the phase transition does not take into account the nature of the coexisting pseudophases. This is not required because coexistence between any pseudophases is expected to vanish in the thermodynamic limit altogether. This does not mean that random cavities become irrelevant in the thermodynamic limit. Rather, the network of many cavities of different sizes, shapes, and affinities are the cause that a small change in $\mu_{\mathrm{A}}$ results only in a small response in $\rho_{\mathrm{A}}$. As demonstrated in section 8.3 the peaks in the OPD that have been attributed to supercritical pseudophases in fact are characterized by an alignment of the mixture particles to the structure of cavities formed by the obstacles.

\subsubsection{Non-random Obstacles}

To demonstrate the importance of randomness in the obstacle positions, systems in which the maximally asymmetric obstacles are arranged on a regular square grid have been investigated. The number of obstacles is then not determined randomly from a Poissonian distribution, but instead determined by the mesh size of the square grid and the system 
size, which must of course be an integer multiple of the mesh size. Since for each system size there is only one configuration of obstacles, taking disorder averages is not required and the simulations are computationally cheap. As demonstrated by the cumulant graph in figure 8.5(a) a critical point with hyperscaling is recovered at some slightly higher fugacity (i.e. a lower temperature than in the pure model without obstacles). As in the case of randomly located symmetric obstacles (chapter 6 ) the critical fugacity increases with $\rho_{\mathrm{O}}$, as shown in figure 8.5(b). Again, at sufficiently high obstacle densities the phase transition must be destroyed (at the very least at $\rho_{\mathrm{O}}=0.5$ where no A particle can be inserted into the system at any location).

\subsection{Structural Properties: Homogeneity Breaking and Do- main Sizes}

To demonstrate the importance of the complicated structure formed by the obstacles and the alignment of the mixture particles to cavities, the WR mixture has been investigated in the microcanonical ensemble, which also allows for much larger system sizes than previously. To simulate the microcanonical ensemble, single particle insertion and removal steps have been used, similar to those described in section 2.2.2.1, but with modified acceptance criteria for the proposed moves: The particle numbers $N_{\mathrm{A}}$ and $N_{\mathrm{B}}$ must stay within a small interval that is typically the size of about $1 \%$ of the actual particle numbers ${ }^{\mathrm{c}}$. All proposed moves that do not leave these intervals and do not lead to an excluded-volume overlap between particles and obstacles are accepted. In the following, only the case with an equal density of $\mathrm{A}$ and $\mathrm{B}$ particles $\rho_{\mathrm{A}}=\rho_{\mathrm{B}}$ is considered. The only thermodynamic parameter left is the total density $\rho=\rho_{\mathrm{A}}+\rho_{\mathrm{B}}$.

Figure 8.6 shows four randomly selected states for the same $\rho_{\mathrm{O}}=0.04$ configuration of maximally asymmetric obstacles at a density $\rho=1.8$. This density is above the critical density $\rho_{\text {cr }} \approx 1.566(3)$ of the pure model ${ }^{38}$, and typical states for the pure model would show a macroscopic demixing (as in the right image of figure 2.1). Instead, states that resemble near-critical fluctuations are seen, i.e. randomly appearing transient domains of various finite sizes that appear in the vicinity of a critical point (c.f. center image of figure 2.1). However, the domains in figure 8.6 differ from critical fluctuations in two key aspects.

The first difference is the size of the domains as a function of the density. In the pure WR mixture, the correlation length diverges with a power law $\sim|t|^{-\nu}$ (with the reduced temperature $t$ defined in equation 3.4) when the critical point is approached from $z_{\mathrm{B}}<z_{\mathrm{B}, \mathrm{cr}}$. Consequently, the domains formed by the near-critical fluctuations

\footnotetext{
'Since no indication of the results' sensitivity to density changes on the order of $1 \%$ were found, these quasi-microcanonical simulations can be considered equivalent to proper microcanonical simulations (for which single particle translations would be adequate Monte Carlo steps).
} 

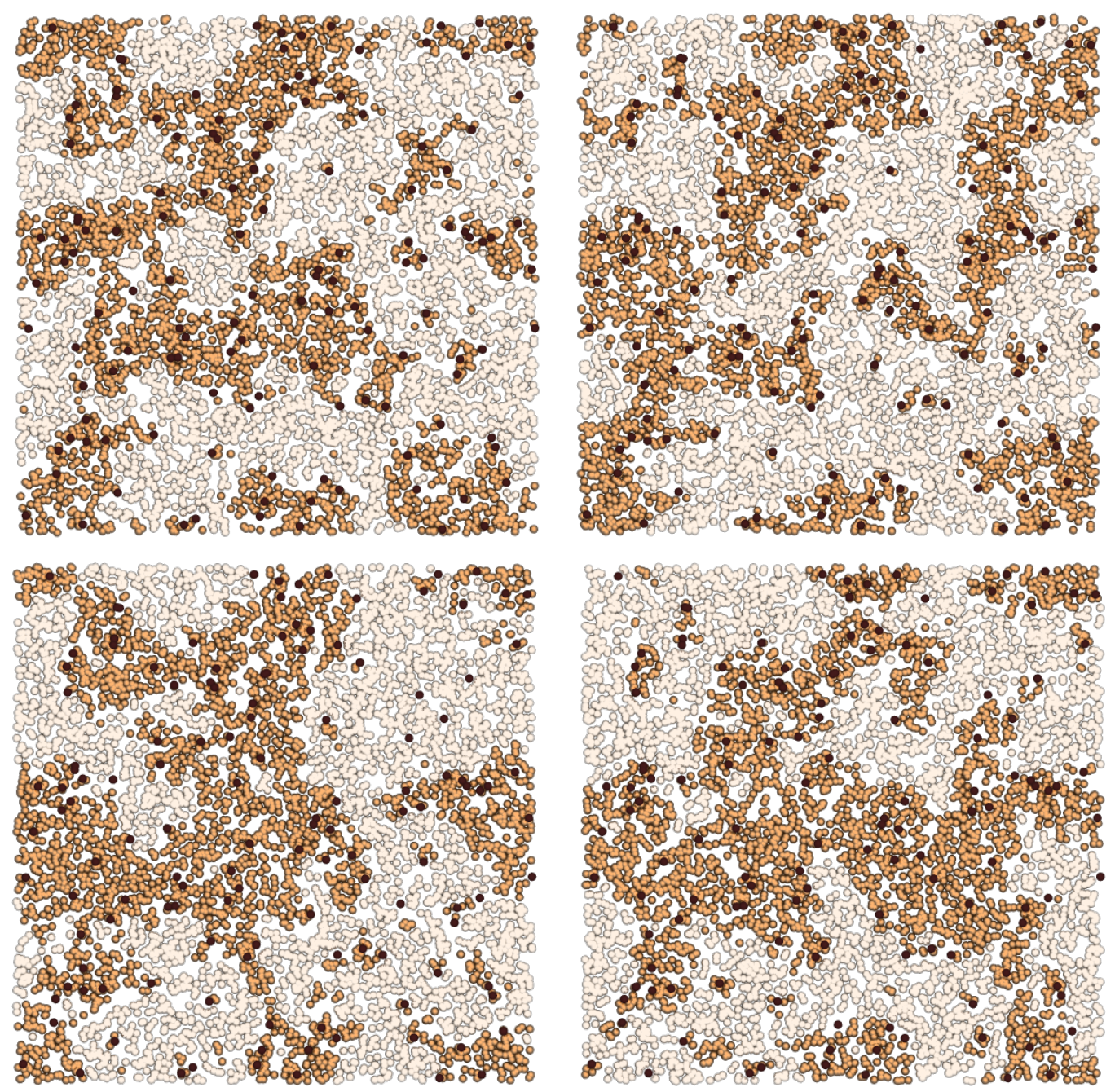

Figure 8.6: Randomly selected microstates of a $60 \times 60 \mathrm{WR}$ system with $\rho_{\mathrm{O}}=0.04$ maximally asymmetric obstacles at density $\rho=1.8$, which is above the demixing density of the pure model. Obstacles are drawn in black. The structures seen visually resemble near-critical fluctuations, but are different in that they are not spatially indifferent (see figure 8.8) and react differently to a density change (figure 8.7). 

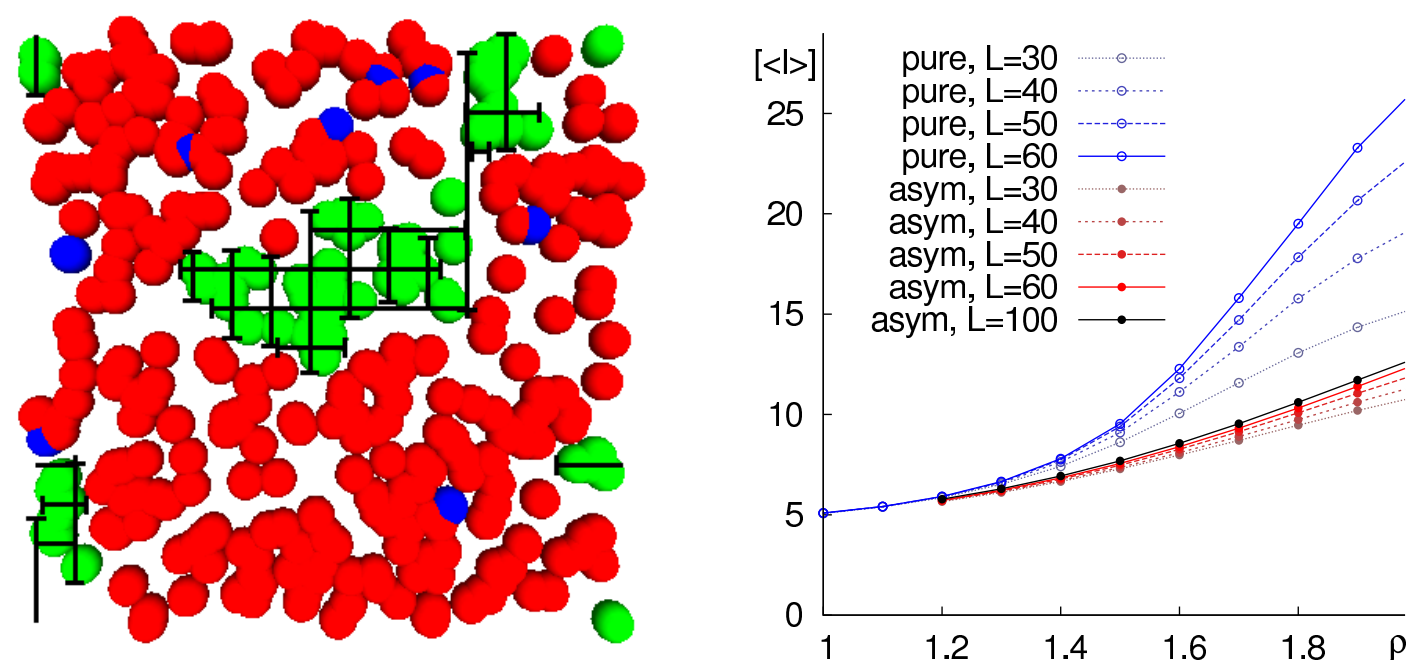

Figure 8.7: Domain sizes as a function of the particle density $\rho$. In the left image, the determination of $P_{i}(l)$ is demonstrated (a definition of this probability distribution is given in the text). Shown is a state of A particles (green), B particles (red), obstacles (blue), and the largest line segments passing through A domains that were found (black). The right plot shows the average lengths of the segments for the pure WR mixture and the WR mixture with $\rho_{\mathrm{O}}=0.04$ maximally asymmetric obstacles as a function of $\rho$. In the pure model, a strong increase around the critical density $\rho_{\text {cr }} \approx 1.56$ is seen, that eventually becomes a divergence for $L \rightarrow \infty$. In the presence of obstacles, the domain sizes appear to remain finite, as required for the absence of a phase transition. 
dramatically grow as the critical point is approached, and ultimately become macroscopic phases at $z_{\mathrm{B}}=z_{\mathrm{B}, \mathrm{cr}}$ (in the simulations this behavior is of course limited by the finite size of the systems). Since in the case of randomly distributed asymmetric obstacles no macroscopic A and B phases coexist in the thermodynamic limit, the typical size of the domains seen in figure 8.6 can not show this type of growth. Consequently, a different growth rate is expected around the critical density. Rather than measuring a correlation length according to some suitable definition, a more direct measure of the domain size has been used.

For a fixed state, horizontal and vertical lines are superimposed on the system. Then, the largest possible line segments passing through an A domains are identified, where such a segment is defined as a maximum interval on the line whose end-points lie on mutually different A particles and which is not crossed by a B particle. The line segments take into account the periodic boundary conditions, i.e. they do not end at the edges of the box but continue on the opposite side. In the extreme case that such a line segment hits itself because of the periodic boundary conditions (because there was no B particle crossing the line at all), it counts as a line segment with size $L$. An example of determining such segments is shown in the left image of figure 8.7. The lengths $l$ of the segments have been collected over many states and for many (300) different obstacle configurations, resulting in probability distributions $P_{i}(l)$ (where again the index $i$ stands for the respective configuration of obstacles). The average segments size $[\langle l\rangle]$ can be considered as a measure for a typical diameter of a domain. The so-defined typical diameters of the domains for the pure WR mixture and the WR mixture with maximally asymmetric obstacles are shown in the right plot of figure 8.7. Around the critical density $\rho \approx 1.56$ the domain sizes behave very differently: While the domain size measured for the pure model increases very strongly (and is restricted from diverging only by the finite size of the system), the domains seen in the case of asymmetric obstacles do not show such a rise.

The other key difference between critical fluctuations and the structures seen in figure 8.6 is that critical fluctuations are spatially indifferent, whereas the structures in the presence of asymmetric obstacles appear at preferred locations. This behavior can be visualized by defining a local measure for a spatial affinity for A particles. To this end, the systems are divided into $L^{2}$ square cells of size one. Then, during the microcanonical simulation for each cell $k$ two counters $C_{k}^{\mathrm{A}}$ and $C_{k}^{\mathrm{B}}$ count how often the cell is found to contain an $\mathrm{A}$ and a $\mathrm{B}$ particle, respectively. To eliminate a possible dependence of these counters from the initial state of the simulation, the counters are collected over 20 independent simulation runs for each obstacle configuration. ${ }^{\mathrm{d}}$ From these counters, the local affinity

\footnotetext{
dThe outcome of this averaging over 20 runs in fact is that there is no dependence on the initial state, anyways.
} 

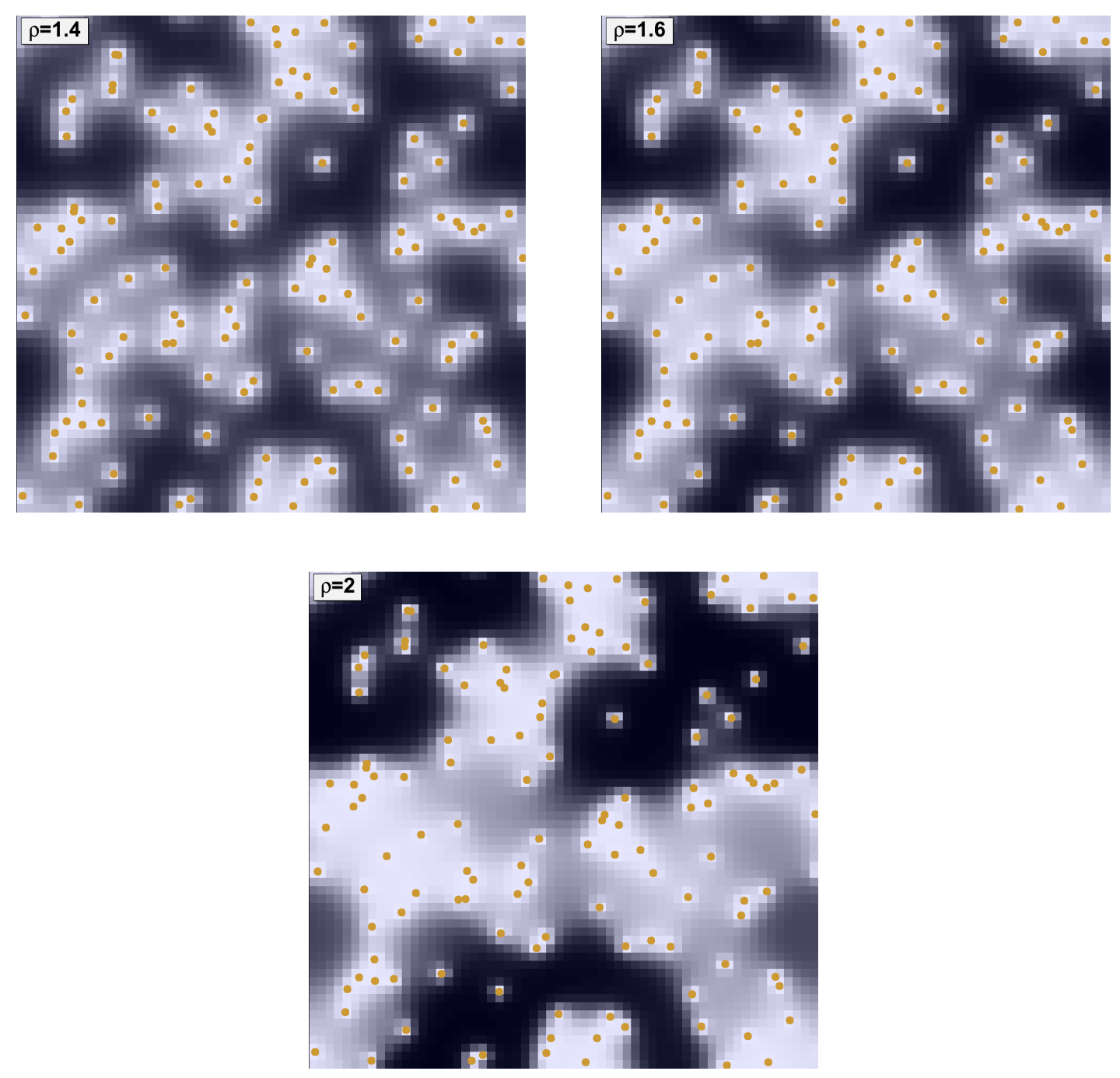

Figure 8.8: Color coded local affinities (equation 8.1) for the system shown in figure 8.6 at different densities $\rho=1.4, \rho=1.8$, and $\rho=2$. Colors range from white $\left(A_{k}=-1\right)$ to black $\left(A_{k}=1\right)$, the orange dots are the obstacles. The image highlights the fate of the structures shown in 8.6: Rather than growing towards macroscopic length scales and causing a phase separation as in the case of the near-critical fluctuations, the structures become more spatially selective and freeze-in to a structure defined by the arrangement of the obstacles. 

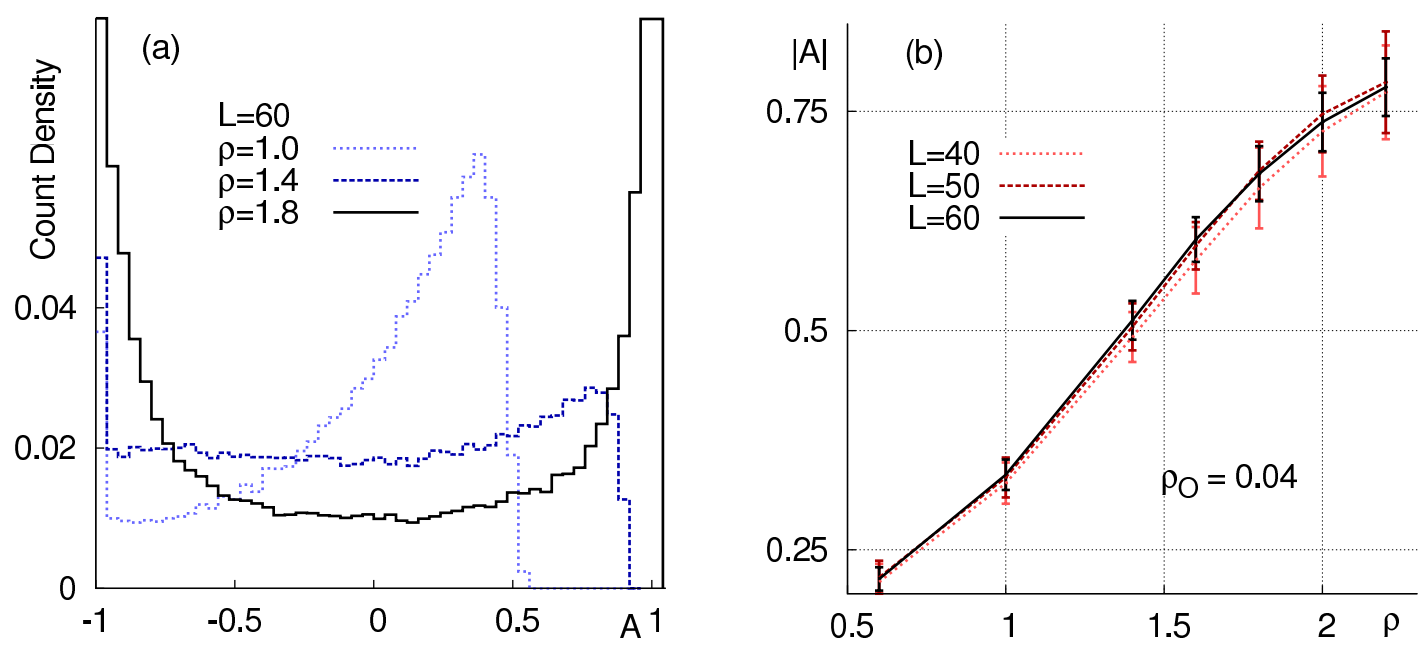

Figure 8.9: Analysis of the inhomogeneity values $A$ defined in equation 8.1. Figure (a) shows histograms of all the inhomogeneity values found for all cells, collected over 40 different obstacle configurations. Around the critical density $\rho_{\mathrm{cr}}=1.56$ of the pure model the shape of the histograms changes from that of a single peak around $A=0$ towards a bimodal shape with peaks at $A \approx \pm 1$. This resembles the behavior of the OPD at a phase transition. Figure (b) shows that despite this behavior, there is no phase transition with an order parameter $|A|$, which is defined as the average absolute value of all $A$ over all obstacle configurations and cells: As a function of $\rho,|A|$ shows an increase that resembles the finite-size behavior of an order parameter, but is insensitive to the system size.

for the A particles can be defined as

$$
A_{k}:=\frac{C_{k}^{\mathrm{A}}-C_{k}^{\mathrm{B}}}{C_{k}^{\mathrm{A}}+C_{k}^{\mathrm{B}}} .
$$

In figure 8.8 the color-coded $A$-values are shown for a configuration with maximally asymmetric obstacles (the same configuration as in figure 8.6) at three different densities. At all three densities, a spatial inhomogeneity caused by the presence of the obstacles is seen. With increasing density these inhomogeneities become more pronounced This effect explains why macroscopic demixing is prevented: When approaching the critical density of the pure model from below, a correlation length develops and small domains appear. In the pure model the size of the domains grows to infinity when approaching the critical point, which ultimately causes the phase transition. When obstacles are present, the domain growth stops at a size scale determined by the obstacles. Upon further increase of the density, the domains freeze into their preferential shapes, rather than continuing to grow.

A more conventional picture of the freezing-in can be obtained by collecting a histogram of all $A_{k}$ values over several obstacle configurations. These histograms for different densities 
are shown in figure 8.9(a) for the case of $\rho_{\mathrm{O}}=0.04$ maximally asymmetric obstacles. Because of the quenched obstacles, some entries for $A_{k} \approx-1$ always exist, which gives the histogram some asymmetry around $A=0$ that is particularly pronounced at low densities, where the expected symmetric peak around $A=0$ is modified to a peak around some $A>0$ with a long tail towards $A=-1$. Apart from this systematic skew, the histograms show a remarkable similarity to the order parameter distributions of systems undergoing a phase transition: At $\rho=1$, a single peak is seen. When the density is increased, this peak broadens and ultimately leads to a bimodal distribution.

This shape of the histograms motivates the question: Does the freezing of the system into domains of a preferential structure constitute a phase transition with the order parameter $|A|$, the average of all $\left|A_{k}\right|$ taken over all cells and all obstacle configurations? According to my simulation data the answer to this question is "probably not". As shown in figure 8.9(b) the value of $|A|$ as a function of $\rho$ does behave like the order parameter in a finite system (c.f. figure 3.2a). But contrary to the order parameter of a phase transition, the curve appears insensitive to the size of the system, and does not seem to converge towards a transition from $|A|=0$ to $|A|>0$ as $L \rightarrow \infty$. The insensitivity of $|A|$ to the system size $L$ suggests that the freezing-in into a preferred domain shape is a gradual process, even in the thermodynamic limit.

\subsection{Real-world Systems: Cell Membranes}

So far, this chapter has dealt with the discussion of the 2D Widom-Rowlinson mixture in the presence of quenched disorder, which has been chosen as a generic model for a binary mixture with short-ranged interactions. Realistic systems that may be considered two dimensional binary mixtures are cell membranes, whose main structure is formed by lipids that have a hydrophilic head group and one or more hydrophobic carbon chain tails. In water these lipids can arrange into the bilayer structure indicated in figure 8.10. Such bilayers can form vesicles of various sizes, and also are the basic structure of cell membranes. Experimental model systems are giant unilamellar vesicles (GUVs) and the related giant plasma membrane vesicles (GPMVs). GUVs are created "from scratch" from dried solutions of lipids and can grow to sizes up to a few tens of micrometers ${ }^{106 ; 107}$, while GPMVs are grown from real biological cell membranes by a chemical process called "blebbing" 108;109 and have typical sizes in the order of ten micrometers ${ }^{81 ; 84}$.

In fluorescence microscopy experiments, a demixing transition has been observed in GPMVs and in GUVs composed of different lipid species ${ }^{81 ; 84}$. Based on the observation of interfaces and near-critical fluctuations it has been argued that this demixing lies in the universality class of the two dimensional Ising model ${ }^{29}$ (as does the demixing in the pure WR mixture). Plasma membranes of real cells on the other hand are not seen to undergo a demixing transition of its lipid components, even under circumstances where attached GPMVs grown from this cell do demix ${ }^{110 ; 111}$. Instead, in eukaryotic cells a heterogeneous 

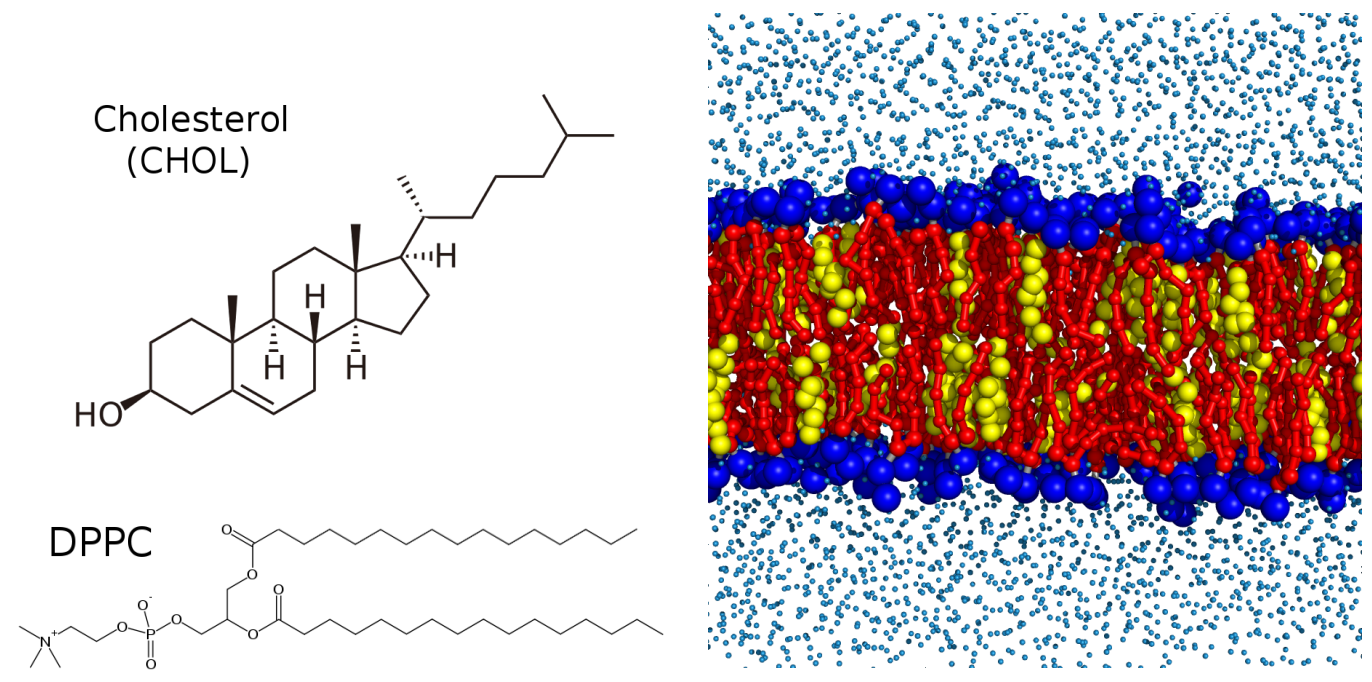

Figure 8.10: Basic structure of a plasma membrane and its constituents. On the left side the chemical structure of cholesterol (CHOL) and dipalmitoyl-phosphatidylcholine (DPPC) are shown (both structures were taken from Wikipedia but are licensed as public domain). In water, DPPC molecules can arrange to form the bilayer structure that is shown in the right image of a computer simulation (imagine the structure to extent into the paper plane). The hydrophilic part of a DPPC head (represented by two blue spheres per molecule) points towards the water (small bright blue spheres). The hydrophobic parts of the DPPC molecule (red) are inside the bilayer and screened from the surrounding water. Cholesterol (yellow) aggregates inside the membrane between the DPPC tails. 
micro- or nanostructure is expected, that is characterized by the appearance of small transient microstructures called lipid rafts $31 ; 32 ; 33$. These rafts are described as regions that, compared to their surroundings, are enriched in cholesterol, and that cluster proteins to work as "signaling platforms" 31 .

It has been proposed that the reason why microdomains instead of a macroscopic demixing is seen in live cells is the presence of static obstacles in the cell membrane that act on the lipid mixture such as random site dilutions ${ }^{30}$. As possible realizations of such obstacles, trans-membrane proteins that are attached to an interior structure of the cell, e.g. the cytoskeleton or an actin network underlying the membrane), were proposed. In such a system, the demixing temperature is lowered (see chapter 6), which is a possible explanation why GPMVs attached to a cell demix while the cell does not. It is however very unlikely that the static obstacles have a completely equal affinity to both of the phases that would form in the free membrane. In fact, according to the raft hypothesis at least the mobile proteins must have a preferred affinity, because otherwise the microdomains could not cluster them together. According to the results in this and the previous chapter, RFIM universality rather than site-diluted Ising universality would be expected, instead. This of course has dramatic consequences for the membrane: Firstly, the presence of asymmetric obstacles should entirely prevent a macroscopic demixing, rather than merely reduce the transition temperature. Secondly, raft-like microstructures would appear that are not critical fluctuations but instead have the characteristics of the RFIM-like domains shown in figure 8.6. That is, they have a reduced sensitivity to temperature (figure 8.7) and appear at preferential locations that are determined by the obstacle configuration.

The assumption that static obstacles are the key mechanism to account for the deviation between model membranes and real cells is compelling but not coercible. Cell membranes ultimately are very complex systems, exhibiting many features not accounted for in the simulations of the WR model in above. First of all, live cells are not in thermal equilibrium, and constant lipid exchange of the membrane with its surrounding may prevent the formation of the large equilibrium domains ${ }^{112}$. Then, the assumption of random locations may not hold. If the obstacles are indeed attached to some interior cell structure then the distribution of obstacles may be not be well described by random obstacles. Some correlated placement of the obstacles may be a better representation of the disorder ensemble ${ }^{111 ; 113}$, which might destroy RFIM universality (as demonstrated in section 8.2.1). Also, while it has been argued that any amount of preference of the obstacles to one of the forming phases should suffice to induce RFIM universality and prevent a demixing transition in the thermodynamic limit, plasma membranes are not infinitely large. Consequently, the originally proposed site-diluted Ising universality ${ }^{30}$ may still be a viable option if the obstacles' asymmetry is so small that a crossover length scale from site-diluted Ising universality to RFIM universality exists that is larger than the size of a cell. Lastly, cell membranes are not flat two dimensional entities, but can bend and fluctuate in the third dimension ${ }^{114}$, which constitutes degrees of freedom that are not captured in the simulations of the $2 \mathrm{D}$ WR model. 


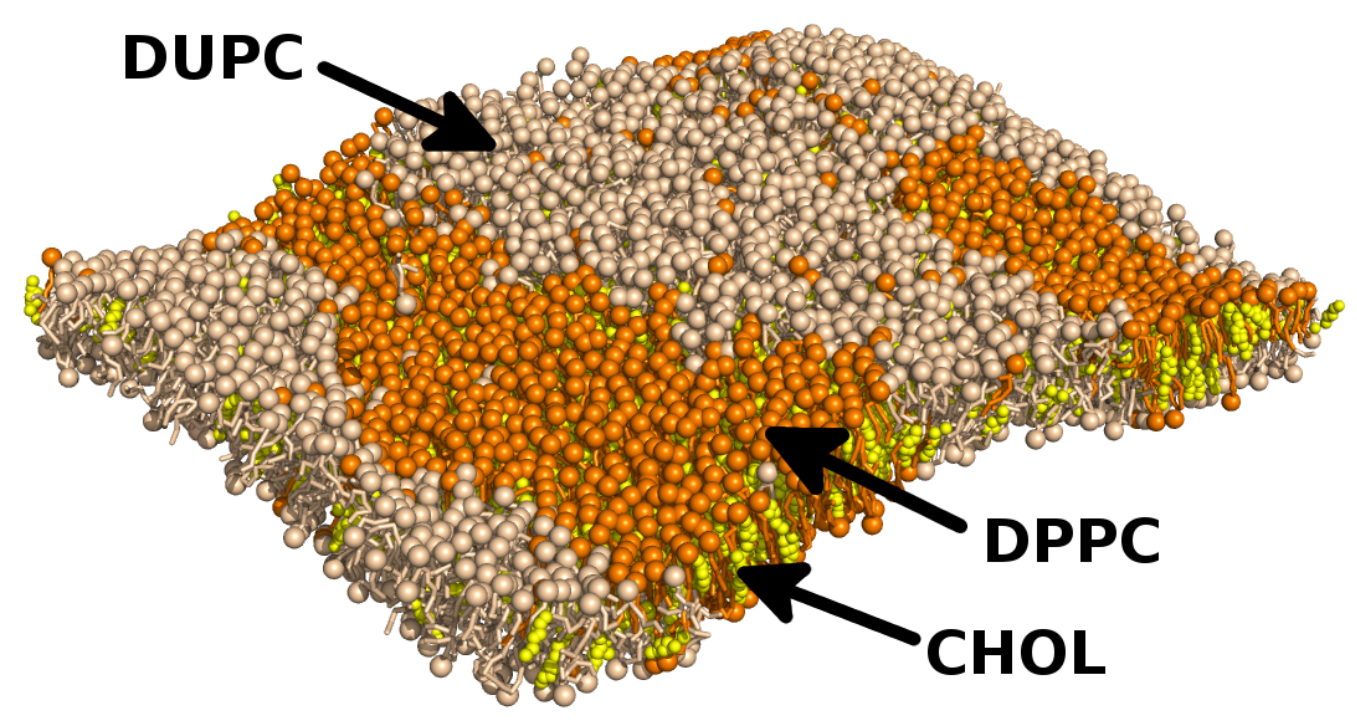

Figure 8.11: Snapshot from a molecular dynamic simulation of a plasma membrane consisting of DPPC, DUPC, and CHOL as described in the text (water not shown). The image shows the state of the membrane $6 \mu$ s after starting from a mixed state. In the foreground, a slab arrangement of a pseudophase consisting of DPPC and CHOL is seen that interfaces a pseudophase consisting of mainly DUPC and almost no CHOL. In the background, a shape that resembles a DPPC droplet is seen. But as shown in figure 8.12, this structure actually is a slab of a CHOL-rich pseudophase. Since a two slab configuration is extremely stable in MD simulations, this state is considered equilibrated and interpreted as an indication that the pure membrane, i.e. without any static obstacles, demixes into two macroscopic phases.

\subsubsection{Molecular Dynamics Simulations of Membranes}

To test if the results found for the Widom-Rowlinson mixture can apply to real cells, a more detailed model has been used to demonstrate that structure formation caused by static obstacles still holds, there. As starting point a GROMACS ${ }^{115 ; 116}$ molecular dynamics (MD) simulation of a membrane bilayer consisting of cholesterol (CHOL) and two different species of phospholipids has been used. The first species is dipalmitoylphosphatidylcholine (see figure 8.10) with fully saturated carbon tails (DPPC), the other species is a modification of the DPPC molecule with modified tail properties that mimic multiple unsaturated bonds (DUPC) ${ }^{117}$. For the representation of the system in the simulation, the MARTINI force field ${ }^{118}$ has been used, which provides a near-atomic resolution of 3-4 atoms per simulation bead. The initial setup of the pure membrane without obstacles was provided by Jelger Risselada, and had been shown to demix into two distinct pseudophases ${ }^{117}$, one consisting mainly of DPPC and CHOL, and the other consisting of mainly DUPC and being lean in CHOL. 


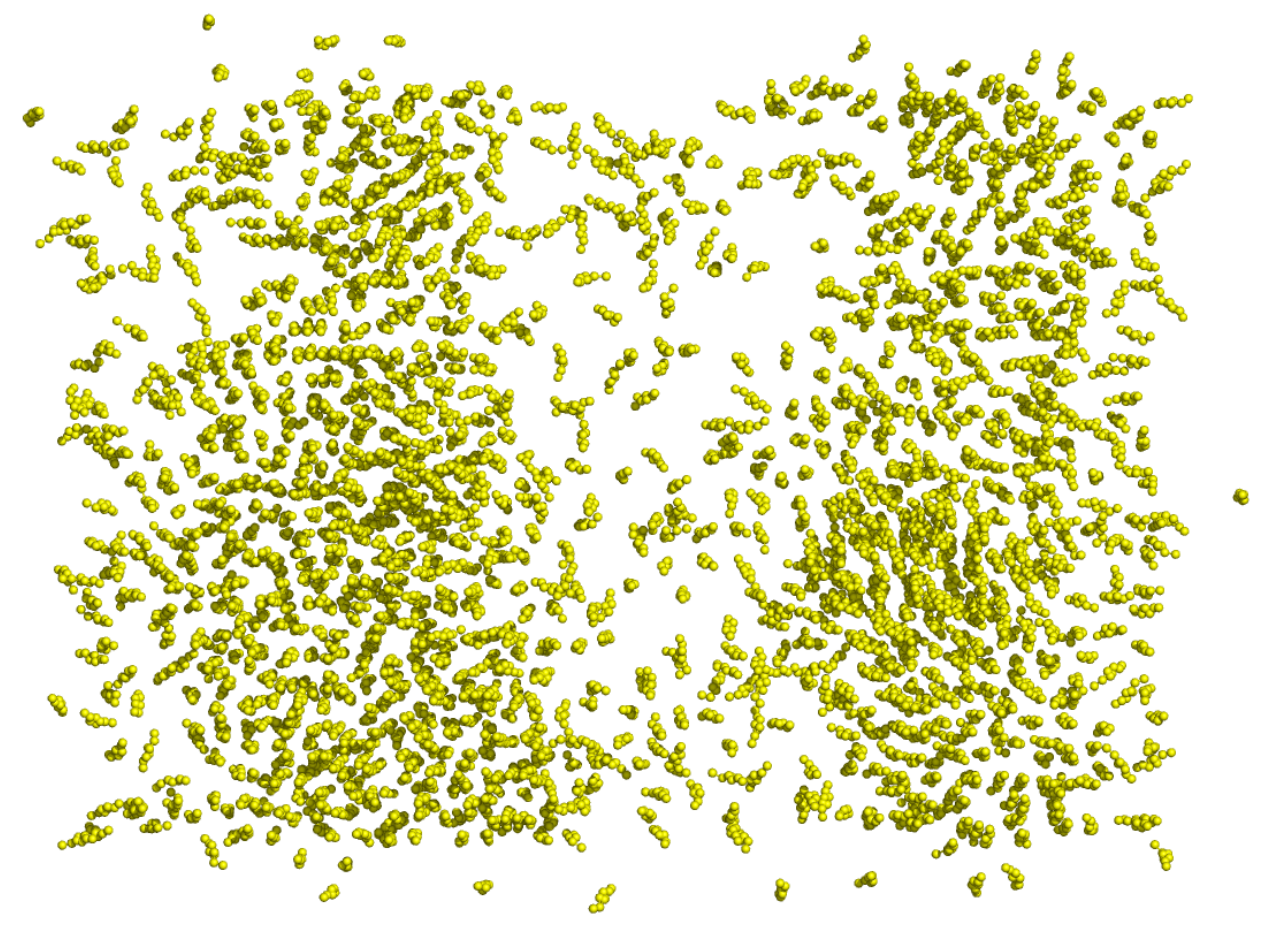

Figure 8.12: The CHOL distribution of the state shown in figure 8.11 as a view from the top onto the membrane surface. The system is in a state consisting of two slabs of a CHOL-rich pseudophase and a CHOL-lean pseudophase. The appearance of slab configurations indicates that in the thermodynamic limit the pseudophases become proper phases, as they are indeed seen in experiments on GUVs and GMPVs. 
This demixing behavior of the "pure membrane" was studied using a $44 \times 29$ nanometer sized membrane patch consisting of 1408 DPPC molecules, 2184 DUPC molecules, and 1532 CHOL molecules. In the initial state, the lipids already form a bilayer but have no specific lateral order, i.e. they are mixed. The simulation of this system is computationally demanding: One microsecond of simulated time requires roughly a week of CPU time on three nodes with eight $2.4 \mathrm{GHz}$ processors each. Consequently, only a single simulation run has been performed. After $6 \mu$ s system time simulated ${ }^{\mathrm{e}}$ this bilayer exhibits the demixing into a pseudophase consisting of mainly DPPC and CHOL, and a pseudophase consisting of mainly DUPC and being depleted of CHOL. The stripe geometry (which is particularly apparent in the distribution of CHOL, cf. figure 8.12) indicates that in the thermodynamic limit these pseudophases become proper macroscopic phases as seen in experiments ${ }^{81 ; 84}$.

From the discussion in chapter 5 only a configuration with a single slab of either pseudophase would be considered a sign of a phase separation. The "two-slab" configuration of figure 8.11 and 8.12 clearly does not minimize the interface between the two coexisting pseudophases. But in an MD simulation, in which particles are restricted to perform physical motions, the situation is slightly different from the Monte Carlo simulations of the WR model: Once the two CHOL slabs are formed they are stable against de-forming, and also prevent that the DUPC lipids separating them can diffuse away. A merging of the two slabs may therefore lie outside of achievable time scales in this simulation. Because of local minimization of the interface between the two pseudophases, the "two-slab" configuration is considered an indication of a phase separation existing in the thermodynamic limit.

\subsubsection{A Membrane with Static Obstacles}

To model the presence of quenched obstacles preferring one of the forming phases a simple choice has been made: For some DPPC molecules, the bead containing the phosphate atom has been immobilized in the lateral direction. These fixed molecules are obstacles as they are immobile with respect to the demixing motion, and they have the same preferential affinity to the pseudophase formed by DPPC and CHOL as a mobile DPPC molecule. On both sides of the bilayer, the same number of obstacles (79 of them) have been used and put on the same locations, which may be considered representing trans-membrane obstacles. The laterally-immobilized beads (and hence the full obstacle) are still allowed to move freely in the z-direction. The membrane can hence still undergo the bending fluctuations that a pure membrane performs. The purpose of preserving these fluctuations is to show that they do not cause incompatibility with the results from the simulation of the WR mixture, in which no analogue of bending exists. Physically, such obstacles can be considered modeling either very long trans-membrane obstacles

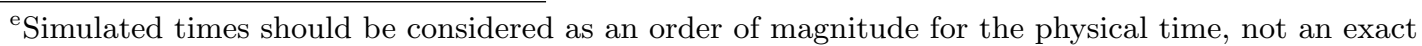
value. It is know that diffusion rates in the MARTINI force field are roughly a factor 4 larger than in atomistic simulations and experiments ${ }^{118}$.
} 
that the membrane fluctuates around, or to represent models that are attached to a very soft underlying network.

In figure 8.13 the state of the membrane with static obstacles after a simulation time of $6 \mu \mathrm{s}$ is shown - the simulation was started in a state in which the lipids are randomly mixed. Clearly, there is no indication of a stripe geometry. Instead, domains of DPPC and CHOL that form around the regions with the highest obstacle density are seen (this is of course equivalent to saying that a DUPC domain arranges such that the obstacles are avoided). The hidden layer of the membrane that is not visible in figure 8.13 looks identical, since it has the same obstacle configuration and a coupling between the layers exists $^{117}$. Figure 8.14 shows a snapshot for the same obstacle configuration, but of an independent simulation run and after $10 \mu \mathrm{s}$. Again, no formation of a stripe that would indicate a macroscopic demixing is found, but instead domains that arrange according to the obstacles are seen.

Because of the extreme amount of CPU time required for the molecular dynamics simulations, thermal averaged inhomogeneities $A_{k}$ according to (8.1) have not been collected. A transition between different domain shapes is not expected to happen at time scales accessible with this simulation. However, a comparison to the inhomogeneities of the Widom-Rowlinson model can be made. To this end, a WR mixture in the microcanonical ensemble has been simulated. The number of $\mathrm{A}$ and $\mathrm{B}$ particles have been chosen to equal the number of DPPC and DUPC particles in a single layer of the membrane. The size of the system has been chosen as $36 \times 24$ resulting in a density $\rho \approx 2$, which is above the critical density of the pure WR mixture. In other words: Since the pure membrane was shown to macroscopically demix, a density in which the pure WR mixture macroscopically demixes has been chosen. The obstacles have been placed at the same lateral locations as in case of the membrane simulation, and the inhomogeneity values $A_{k}$ according to 8.1 have been obtained.

Figure 8.15 shows the color-coded inhomogeneities for the two different obstacle choices that were used previously (the maximally asymmetric obstacles and the semidiluted obstacles). By definition of $A_{k}$, the images show the preferred regions for the formation of A domains (dark regions, corresponding to DUPC domains) and B domains (bright regions, corresponding to DPPC and CHOL domains $)^{\mathrm{f}}$. Qualitatively, the preferred domains are the same for both cases of obstacles; the preferential locations for the maximally asymmetric obstacles are merely "sharper". Visual comparison shows that the domain structures seen in the MD simulations (figures 8.13 and 8.14) are in agreement with the preferred locations in figure 8.15. This can be considered as a sign that the results found for the WR mixture (the prevention of a macroscopic demixing and the appearance of domains that appear at preferred locations) also hold in more realistic membrane models and possibly for real plasma membranes' ${ }^{\mathrm{g}}$.

\footnotetext{
${ }^{\mathrm{f}}$ Note that individual domains do not necessarily look identical to the shapes of preferred regions.

${ }^{g}$ But real membranes are much more complex than the membrane model being used here.
} 

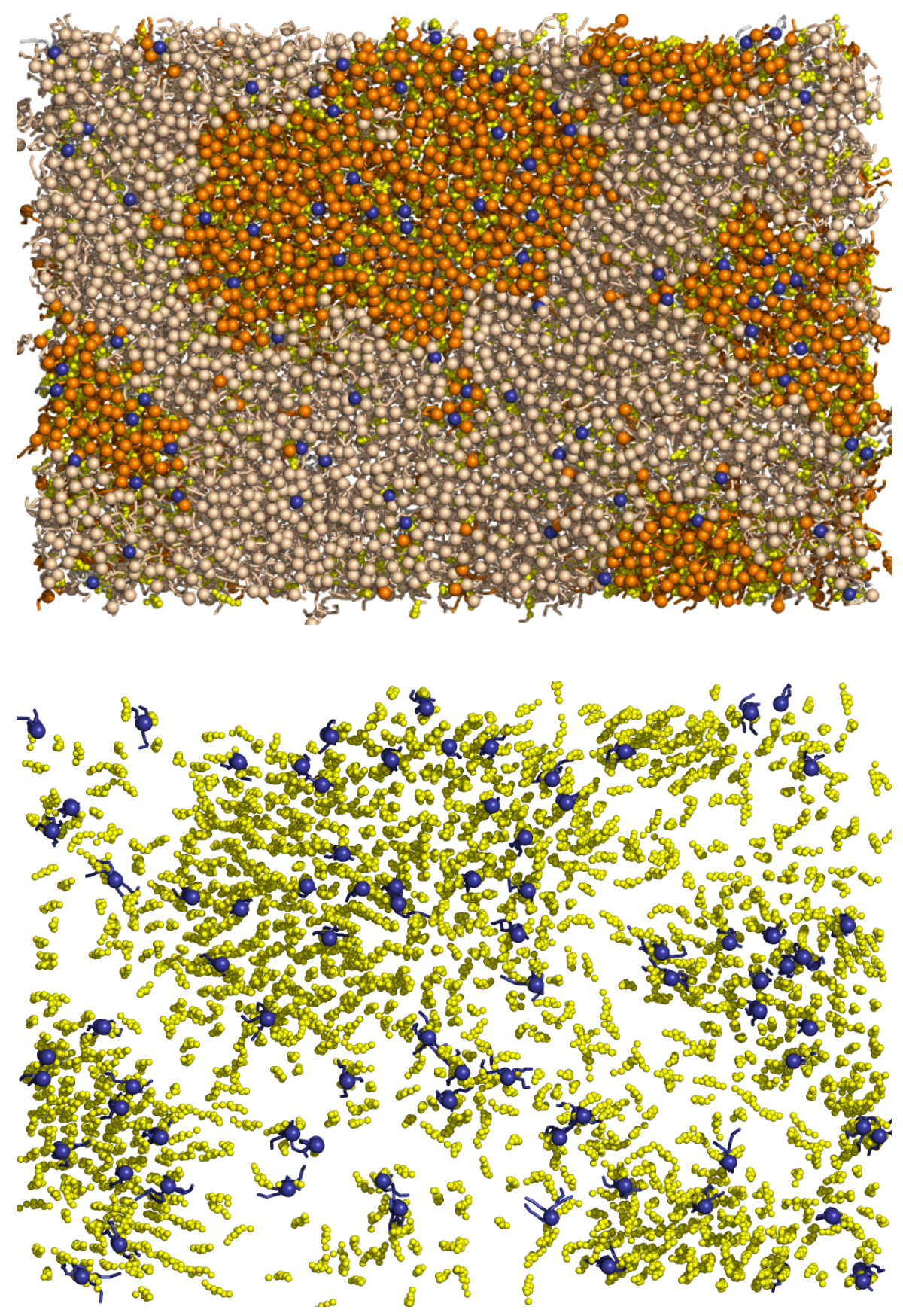

Figure 8.13: Simulation results for the membrane configuration shown in figure 8.11 and 8.12, but with static obstacles (blue). The images show the state of the membrane $6 \mu$ s after starting in a mixed initial state. The upper image shows the top view of the membrane, where the head group of each phospholipid is represented with a single sphere. The CHOL, which resides inside the bilayer (c.f. figure 8.10), is shown separately in the lower image. The slab configuration indicating a macroscopic demixing that was found for the pure membrane (figures 8.11 and 8.12) does not appear anymore. Instead, small domains appear that are determined by the obstacle configuration. The location of the domains is in good agreement with predictions from Monte Carlo simulations (figure 8.15). 

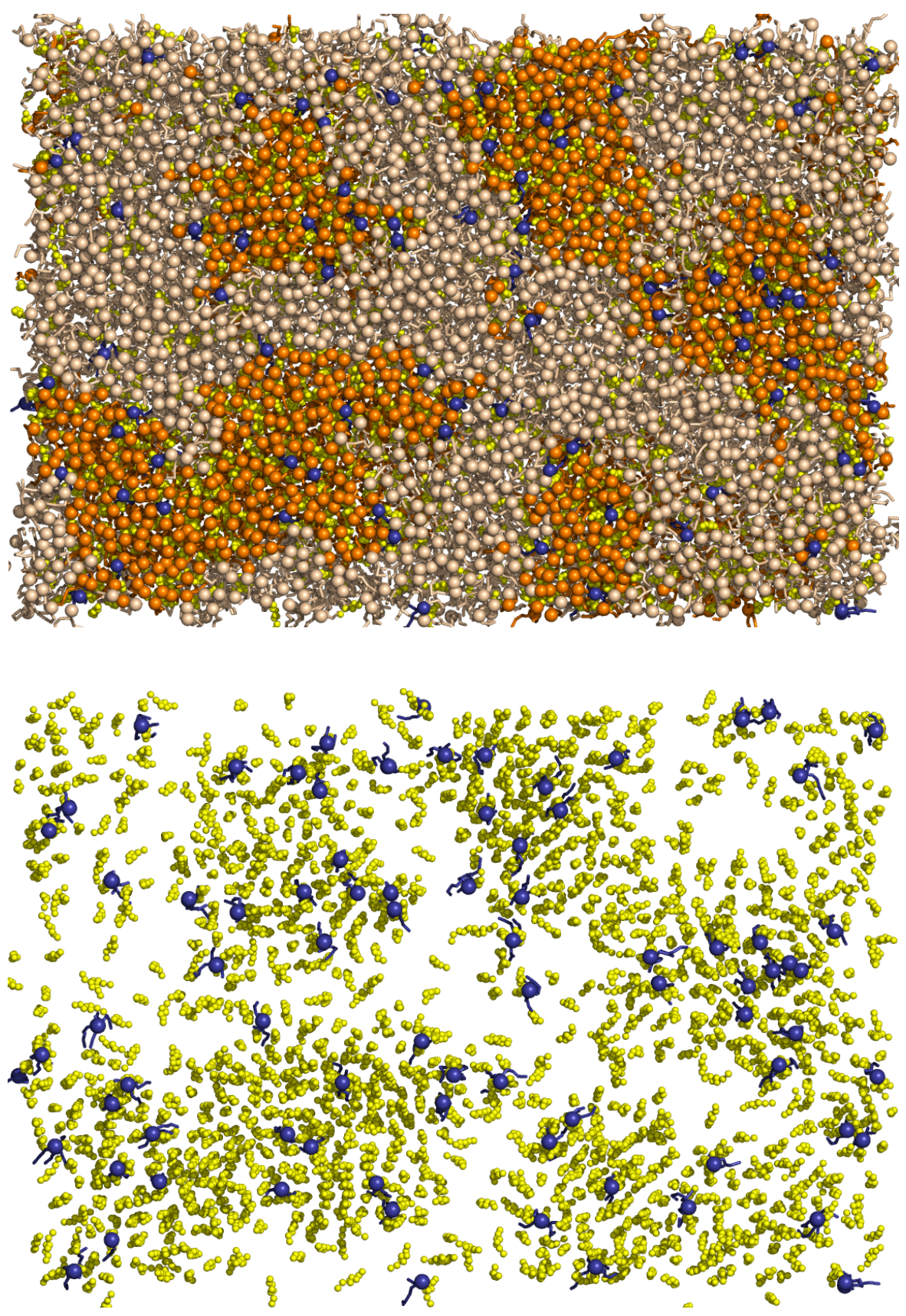

Figure 8.14: Analogue of figure 8.13, but from a simulation performed with a different set of random numbers and after a simulation over $10 \mu \mathrm{s}$. Again, the slab geometry is eliminated in favor of a domain structure. As in figure 8.13 the locations of the domains compare well to the Monte Carlo results in figure 8.15. Note that even though the head groups of the obstacle molecules are laterally fixed, their tails may freely move within this constraint. Due to periodic boundary conditions, obstacle molecules close to the boundary can therefore appear at the opposite side of the simulation box (compared to figure 8.13). 

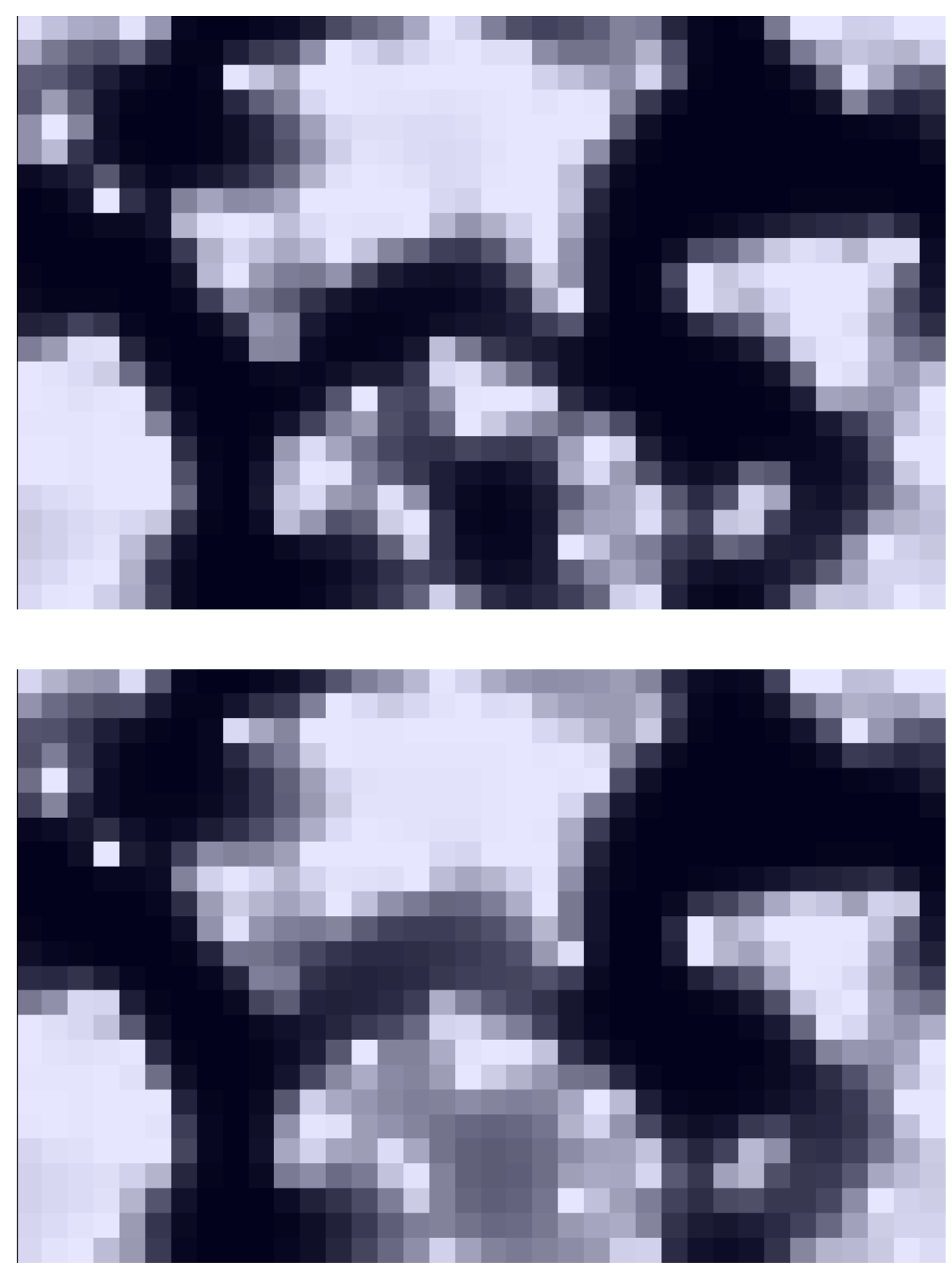

Figure 8.15: Monte Carlo simulation of a $36 \times 24$ two-dimensional Widom-Rowlinson model with $N_{\mathrm{A}}$ and $N_{\mathrm{B}}$ equal to half the number of mobile DUPC and DPPC molecules used in the MD simulations, respectively. Obstacles have been placed at the (lateral) locations that DPPC head beads were fixed to in the MD simulation The upper and the lower image show the color-coded $A_{k}$ values for the case of maximally asymmetric obstacles and semidiluted obstacles, respectively. Darker and brighter regions show a preference for A and B particles, respectively (c.f. figure 8.8). In both cases of obstacles, clear preferential locations for domains are seen, which coincide well with the locations where the domains are seen in figure 8.13 and figure 8.14. 


\subsection{Summary: Elimination of a Phase Transition}

In this chapter, the two dimensional Widom-Rowlinson model in the presence of asymmetric obstacles has been investigated for two different types of obstacles. Consistent with simulations of similar lattice based systems ${ }^{28}$, coexistence between pseudophases has been found, but at even higher fugacities than in case of symmetric obstacles. By considering the finite-size dependence of this coexistence region it has been argued that in the thermodynamic limit no phase coexistence and thus no phase transition between a liquid and a gas phase exists for any finite fugacity. Since the WR model is in the same universality class as fluids undergoing a liquid $\leftrightarrow$ gas phase transition this suggests that two dimensional fluids in the presence of quenched disorder do not have a liquid or a gas phase at any non-zero temperature. Similarly, two-dimensional binary mixtures with short-ranged interactions in the presence of randomly placed obstacles with an unequal coupling to the particle species should not macroscopically demix at finite temperatures. In combination with the results of chapter 6 it has been shown that for the elimination of the phase transition two ingredients are both required: an asymmetry in the interaction of the obstacles and a sufficient randomness in their distribution.

By simulation in the microcanonical ensemble the formation of finite domains has been found. These domains were shown to be transient at temperatures not too far below the critical point of the pure model, but spatially selective. It has been shown that by cooling the system the spatial selectivity of the domains becomes more pronounced, and the particles "freeze-in" to a structure determined by the obstacles — such a freezing also explains the absence of a macroscopic demixing. By considering simulation results for different system sizes it has been argued that this freezing into a preferred structure is a gradual process, and does not constitute a phase transition by itself.

Finally, plasma membranes of cells have been briefly introduced as an example of a real-world system. It has been argued that with respect to lateral arrangement of their constituting lipids they may be considered a binary mixture in the presence of quenched disorder. Molecular dynamics simulations of a model membrane with nearatomic resolution have been performed. As predicted by the results for the WidomRowlinson model, an absence of a macroscopic demixing in favor of the formation of spatially selective domains has been found. The elimination of macroscopic demixing is in agreement with experimental results, in which no demixing transition is observed in live eukaryotic cells; the small domains enriched in cholesterol that were found are compatible with the lipid raft hypothesis. A feature predicted and found is the spatial selectivity for the cholesterol rich domains, which not a property usually attributed to lipid rafts. But in view of their predicted pivotal role in signaling processes, spatial selectivity may even be an important property of lipid rafts, as it allows to control the formation of domains via an adjustment of the obstacle configuration. 


\section{Summary}

In this work, the universal behavior of the liquid $\leftrightarrow$ gas transition in the presence of random static inhomogeneities ("obstacles") has been investigated by means of Monte Carlo computer simulations. To this end, state of the art Monte Carlo sampling and finite-size scaling techniques (one of which has been used for the first time in literature $^{24 ; 100}$ ) have been employed. By the use of efficient simulation techniques and a high level of automatization, it has been possible to consider thousands of different disorder configurations for a given system size. As demonstrated by the results, this is crucial for a reliable analysis (figure 7.1).

For obstacles that are arranged on a regular grid it has been found that there still exists a liquid $\leftrightarrow$ gas phase transition. The critical temperature is shifted to lower temperatures for an increasing amount of obstacles (figure 8.5). If instead the obstacles are randomly distributed, the behavior of the fluid is determined by the symmetry of the obstacles' interactions with the liquid and the gas phase and the spatial dimensionality.

Fluids with obstacles whose interactions are indifferent to the fluid's phase have been investigated in chapter 6 . It has been demonstrated that such a system behaves like a sitediluted Ising model. This implies that for low concentrations of obstacles the liquid $\leftrightarrow$ gas transition still exists, but at a critical temperature that is shifted towards lower values by an amount that is proportional to the concentration of obstacles (figure 6.6).

If the obstacles have a preferred affinity to either the liquid or the gas phase, a behavior that is consistent with that of the random-field Ising model is seen. In a three dimensional system (chapter 7 ) this implies that the critical exponents of the liquid $\leftrightarrow$ gas transition not only differ from the "standard" Ising values ${ }^{7}$. It also requires one to introduce a new critical exponent, the violation of hyperscaling exponent $\theta$. This new critical exponent causes an unconventional system behavior at the critical point that is usually expected at a first order phase transition, namely the existence of a free energy barrier between coexisting liquid and gas states that diverges with the system size. The value of the hyperscaling exponent has been approximately determined (in agreement with later and more precise results ${ }^{9}$ ) and demonstrated to be distinctively non-zero.

The case of the two dimensional fluid in the presence of static obstacles that are sensitive to the phases has been investigated in chapter 8. By using finite-size scaling it has been demonstrated that the simulated systems show signatures of a liquid $\leftrightarrow$ gas phase 
transition, but that the apparent critical temperature strongly depends on the system size. The system in the thermodynamic limit has been argued not to undergo a phase transition, but instead on large scales to be on a supercritical-like state characterized by an obstaclegenerated spatial inhomogeneity. It has been shown that this inhomogeneity structure becomes increasingly pronounced as temperature is decreased (thereby countering the phase transition), but that this "alignment" to the obstacle structure does not constitute a phase transition itself (see figure 8.9).

Since the fluid that has been investigated, the Widom-Rowlinson fluid, has an interpretation as a binary mixture with short ranged interactions, the results found for the fluid are expected to hold for binary mixtures, too. That is, mixtures in the presence of randomly located static obstacles that have an equal interaction with both particle species are expected to behave like a site-diluted Ising model, whereas obstacles that have a stronger affinity to one of the species cause a random-field like behavior. This does in particular imply that two dimensional mixtures in the presence of such obstacles do not macroscopically demix, but instead arrange according to preferential microstructures as the temperature is decreased.

A two dimensional mixture system where these finding may be applicable has been briefly discussed: The plasma membranes of eukaryotic cells. Molecular dynamics simulations of a membrane model with two different species of phospholipids and static inhomogeneities with a preference for one of the lipids species have been performed. The structures found in those simulation were shown to be consistent with the structures expected from the simulations of the Widom-Rowlinson mixture. By extending a hypothesis of Yethiraj ${ }^{30}$ it has been argued that plasma membranes may exhibit random-field like behavior owing to the presence of trans-membrane proteins coupled to the cytoskeleton. This would explain the apparent absence of phospholipid demixing (that is seen in experiments on cytoskeleton-free model membranes) in live cells as a universal behavior that does not depend on the detailed interactions of the membrane components. The small and transient domains that have been seen in the simulation of the Widom-Rowlinson mixture (figure 8.6) have been argued to be compatible with the hypothetical lipid rafts ${ }^{31}$. If lipid rafts can indeed be understood as a random-field like fluctuation, this implies that the regions where rafts occur are determined by the obstacle configuration. Assuming that in a cell the obstacle configuration is fixed by the cytoskeleton, this may provide a mechanism for the cell interior to influence the spatial arrangement in the cell membrane. 


\section{Derivatives of the Free Energy}

\section{Definitions}

Consider a system with a variable number of particles, a conjugate field $\mu$ to the particle number $N$, a tuple $\mathbf{o}$ of other state variables that are held constant, and $\beta=1$ (for simplicity). The probability to find a this system in a state with $N$ particles (the order parameter distribution) is given by

$$
P(N \mid \mu, \mathbf{o})=\frac{1}{\mathcal{Z}(\mu, \mathbf{o})} Z(N, \mathbf{o}) e^{\mu N}
$$

where

$$
\mathcal{Z}(\mu, \mathbf{o})=\sum_{N=0}^{\infty} Z(N, \mathbf{o}) e^{\mu N}
$$

is the grand partition function, and $Z(N, \mathbf{o})$ is the partition function for the system in case of a constant number of $N$ particles. The free energy of the system is given by

$$
F(\mu, \mathbf{o})=-\log \mathcal{Z}(\mu, \mathbf{o})
$$

Ensemble averages of functions $f(N)$ can be expressed as

$$
\langle f\rangle:=\sum_{N=0}^{\infty} f(N) P(N \mid \mu)=\frac{1}{\mathcal{Z}(\mu, \mathbf{o})} \sum_{N=0}^{\infty} f(N) Z(N, \mathbf{o}) e^{\mu N}
$$




\section{Derivatives of Observables}

For derivatives of the grand partition function and ensemble averages of functions of type $f(N)$ with respect to $\mu$ the following rules hold true:

$$
\begin{aligned}
& \partial_{\mu} \mathcal{Z}(\mu, \mathbf{o}) \quad \stackrel{(A .2)}{=} \quad \sum_{N=0}^{\infty} Z(N, \mathbf{o}) \underbrace{\partial_{\mu} e^{\mu N}}_{=N e^{\mu N}} \stackrel{(A .4)}{=} \mathcal{Z}\langle N\rangle \\
& \partial_{\mu}\langle f\rangle \stackrel{(A .4)}{=} \quad \partial_{\mu}\left[\frac{1}{\mathcal{Z}} \sum_{N=0}^{\infty} Z(N, \mathbf{o}) f(N) e^{\mu N}\right] \\
& =\quad \frac{1}{\mathcal{Z}} \sum_{N=0}^{\infty} Z(N, \mathbf{o}) f(N) \partial_{\mu} e^{\mu N}-\frac{1}{\mathcal{Z}^{2}}\left(\partial_{\mu} \mathcal{Z}\right) \sum_{N=0}^{\infty} \rho(N, \ldots) f(N) e^{\mu N} \\
& \stackrel{(A .4, A .5)}{=}\langle N f\rangle-\langle N\rangle\langle f\rangle
\end{aligned}
$$

\section{Derivatives of the Free Energy}

The repeated usage of the equations (A.5) and (A.6) allows to straightforwardly express the first derivatives of $\log \mathcal{Z}$ (i.e. those of the free energy) with respect to $\mu$ as a function of the first moments of the order parameter distribution:

$$
\begin{aligned}
\frac{\partial \log \mathcal{Z}(\mu, \mathbf{o})}{\partial \mu} & =\frac{1}{\mathcal{Z}} \partial_{\mu} \mathcal{Z} \stackrel{(A .5)}{=}\langle N\rangle \\
\frac{\partial^{2} \log \mathcal{Z}(\mu, \mathbf{o})}{\partial \mu^{2}} & =\partial_{\mu}\langle N\rangle \stackrel{(A .6)}{=}\left\langle N^{2}\right\rangle-\langle N\rangle^{2} \\
\frac{\partial^{3} \log \mathcal{Z}(\mu, \mathbf{o})}{\partial \mu^{3}} & =\partial_{\mu}\left[\left\langle N^{2}\right\rangle-\langle N\rangle^{2}\right] \stackrel{(A .6)}{=}\left\langle N^{3}\right\rangle-\langle N\rangle\left\langle N^{2}\right\rangle-2\langle N\rangle\left(\left\langle N^{2}\right\rangle-\langle N\rangle^{2}\right) \\
& =\left\langle N^{3}\right\rangle-3\left\langle N^{2}\right\rangle\langle N\rangle+2\langle N\rangle^{3} \\
\frac{\partial^{4} \log \mathcal{Z}(\mu, \mathbf{o})}{\partial \mu^{4}} & =\partial_{\mu}\left[\left\langle N^{3}\right\rangle-3\left\langle N^{2}\right\rangle\langle N\rangle+2\langle N\rangle^{3}\right] \\
& \stackrel{(A .6)}{=}\left\langle N^{4}\right\rangle-\langle N\rangle\left\langle N^{3}\right\rangle-3\left(\left\langle N^{3}\right\rangle-\langle N\rangle\left\langle N^{2}\right\rangle\right)\langle N\rangle \\
& -3\left\langle N^{2}\right\rangle\left(\left\langle N^{2}\right\rangle-\langle N\rangle^{2}\right)+6\langle N\rangle^{2}\left(\left\langle N^{2}\right\rangle-\langle N\rangle^{2}\right) \\
= & \left\langle N^{4}\right\rangle-4\left\langle N^{3}\right\rangle\langle N\rangle+3\left\langle N^{2}\right\rangle^{2}+12\left\langle N^{2}\right\rangle\langle N\rangle^{2}-6\langle N\rangle^{4}
\end{aligned}
$$




\section{Bibliography}

[1] H. E. Stanley. Introduction to Phase Transitions and Critical Phenomena. Oxford University Press, July 1987. 1, 22

[2] J. V. Sengers and J. M. H. L. Sengers. Thermodynamic Behavior of Fluids Near the Critical Point. Annual Review of Physical Chemistry, 37:189-222, October 1986. 1

[3] N. Goldenfeld. Lectures on Phase Transitions and the Renormalization Group. Addison-Wesley, Advanced Book Program, Reading, Mass., 1992. 1, 25, 29

[4] Marcos Ley-Koo and Melville S. Green. Revised and extended scaling for coexisting densities of $\mathrm{SF}_{6}$. Phys. Rev. A, 16:2483-2487, 1977. 1

[5] Charles Pittman, Theodore Doiron, and Horst Meyer. Equation of state and critical exponents of ${ }^{3} \mathrm{He}$ and a ${ }^{3} \mathrm{He}-{ }^{4} \mathrm{He}$ mixture near their liquid-vapor critical point. Phys. Rev. B, 20:3678-3689, 1979. 1

[6] J. Cardy. Scaling and Renormalization in Statistical Physics. Cambridge University Press, April 1996. 1, 65

[7] T. Nattermann. Theory of the Random Field Ising Model. eprint arXiv:condmat/9705295, May 1997. 1, 75, 80, 111

[8] D. P. Belanger and A. P. Young. The random field Ising model. Journal of Magnetism and Magnetic Materials, 100(1-3):272-291, 1991. 1, 2, 3, 75

[9] L. A. Fernandez, V. Martin-Mayor, and D. Yllanes. Critical behavior of the dilute antiferromagnet in a magnetic field. Phys. Rev. B, 84:100408, September 2011. 1, 86,111

[10] Michael Aizenman and Jan Wehr. Rounding of first-order phase transitions in systems with quenched disorder. Phys. Rev. Lett., 62:2503-2506, May 1989. 1, 87

[11] Dietrich Stauffer and Amnon Aharony. Introduction to percolation theory; rev. version. Taylor and Francis, London, 1991. 1

[12] S. S. Kistler. Coherent Expanded-Aerogels. The Journal of Physical Chemistry, 36(1):52-64, 1931. 1

[13] P. G. De Gennes. Liquid-liquid demixing inside a rigid network. Qualitative features. The Journal of Physical Chemistry, 88(26):6469-6472, December 1984. 2, 75, 86

[14] L. D. Gelb, K. E. Gubbins, R. Radhakrishnan, and M. Sliwinska-Bartkowiak. Phase separation in confined systems. Reports on Progress in Physics, 62:1573-1659, December 1999. 2 
[15] Apollo P. Y. Wong and M. H. W. Chan. Liquid-vapor critical point of ${ }^{4} \mathrm{He}$ in aerogel. Phys. Rev. Lett., 65:2567-2570, 1990. 2

[16] A. P. Y. Wong, S. B. Kim, W. I. Goldburg, and M. H. W. Chan. Phase separation, density fluctuation, and critical dynamics of $\mathrm{N}_{2}$ in aerogel. Phys. Rev. Lett., 70:954-957, 1993. 2

[17] Abel Weinrib and B. I. Halperin. Critical phenomena in systems with long-rangecorrelated quenched disorder. Phys. Rev. B, 27:413-427, January 1983. 2

[18] B. Widom and J. S. Rowlinson. New Model for the Study of Liquid-Vapor Phase Transitions. The Journal of Chemical Physics, 52(4):1670-1684, 1970. 2, 5, 7, 10

[19] Michael E. Fisher and Michael N. Barber. Scaling Theory for Finite-Size Effects in the Critical Region. Phys. Rev. Lett., 28(23):1516-1519, June 1972. 2, 25

[20] Y. Imry and D. Bergman. Critical Points and Scaling Laws for Finite Systems. Phys. Rev. A, 3:1416-1418, April 1971. 2

[21] G. Orkoulas, A. Z. Panagiotopoulos, and Michael E. Fisher. Criticality and crossover in accessible regimes. Phys. Rev. E, 61(5):5930-5939, May 2000. 2, 19, 31, 32

[22] G. Orkoulas, Michael E. Fisher, and A. Z. Panagiotopoulos. Precise simulation of criticality in asymmetric fluids. Phys. Rev. E, 63(5):051507, April 2001. 2, 19, 31, 32

[23] K. Binder. Finite size scaling analysis of ising model block distribution functions. Zeitschrift fur Physik B Condensed Matter, 43:119-140, June 1981. 2, 25, 29

[24] R. L. C. Vink, T. Fischer, and K. Binder. Finite-size scaling in Ising-like systems with quenched random fields: Evidence of hyperscaling violation. Phys. Rev. E, 82(5):051134, November 2010. 3, 43, 79, 80, 81, 82, 83, 85, 111

[25] R. L. C. Vink, K. Binder, and H. Löwen. Critical Behavior of Colloid-Polymer Mixtures in Random Porous Media. Phys. Rev. Lett., 97(23):230603, December 2006. 3,80

[26] R. L. C. Vink, K. Binder, and H. Löwen. Colloid-polymer mixtures in random porous media: finite size scaling and connected versus disconnected susceptibilities. Journal of Physics: Condensed Matter, 20(40):404222+, October 2008. 3, 41, 43, 77,86

[27] M. Álvarez, D. Levesque, and J. J. Weis. Monte Carlo approach to the gas-liquid transition in porous materials. Phys. Rev. E, 60:5495-5504, November 1999. 3, 79

[28] Jordi Gomez, Francesc Sagues, and Ramon Reigada. Effect of integral proteins in the phase stability of a lipid bilayer: Application to raft formation in cell membranes. The Journal of Chemical Physics, 132(13):135104, 2010. 3, 110 
[29] Aurelia R. Honerkamp-Smith, Pietro Cicuta, Marcus D. Collins, Sarah L. Veatch, Marcel den Nijs, M. Schick, and Sarah L. Keller. Line Tensions, Correlation Lengths, and Critical Exponents in Lipid Membranes Near Critical Points. Biophysical Journal, 95(1):236-246, 2008. 3, 100

[30] Arun Yethiraj and James C. Weisshaar. Why Are Lipid Rafts Not Observed In Vivo? Biophysical Journal, 93(9):3113-3119, November 2007. 3, 102, 112

[31] K. Simons and E. Ikonen. Functional rafts in cell membranes. Nature, 387:569-572, June 1997. 4, 102, 112

[32] Sean Munro. Lipid Rafts: Elusive or Illusive? Cell, 115(4):377-388, 2003. 4, 102

[33] Daniel Lingwood and Kai Simons. Lipid Rafts As a Membrane-Organizing Principle. Science, 327(5961):46-50, January 2010. 4, 102

[34] E. de Miguel, N. G. Almarza, and G. Jackson. Surface tension of the WidomRowlinson model. Journal of Chemical Physics, 127(3), July 2007. 6, 10

[35] E. Bergmann. Scaled particle theory for non-additive hard spheres. Molecular Physics, 32:237-256, July 1976. 10, 11

[36] Matthias Schmidt. Density functional for the Widom-Rowlinson model. Phys. Rev. E, 63(1):010101, December 2000. 10

[37] C. Y. Shew and A. Yethiraj. Phase behavior of the Widom-Rowlinson mixture. Journal of Chemical Physics, 104(19), 1996. 10

[38] Gregory Johnson, Harvey Gould, J. Machta, and L. K. Chayes. Monte Carlo Study of the Widom-Rowlinson Fluid Using Cluster Methods. Phys. Rev. Lett., 79(14):2612-2615, October 1997. 10, 17, 94

[39] David Ruelle. Existence of a Phase Transition in a Continuous Classical System. Phys. Rev. Lett., 27(16):1040-1041, October 1971. 10

[40] R. L. C. Vink. Critical behavior of the Widom-Rowlinson mixture: coexistence diameter and order parameter. Journal of Chemical Physics, 124:094502, 2006. 10

[41] A. Buhot. Cluster algorithm for nonadditive hard-core mixtures. Journal of Chemical Physics, 122(2), 2005. 10

[42] Ronald Dickman and George Stell. Critical Behavior of the Widom-Rowlinson Lattice Model. Journal of Chemical Physics, 102(21), February 1995. 10

[43] Marjolein Dijkstra. Phase behavior of nonadditive hard-sphere mixtures. Phys. Rev. E, 58(6):7523-7528, December 1998. 11 
[44] R. Zwanzig. First-Order Phase Transition in a Gas of Long Thin Rods. Journal of Chemical Physics, 39:1714-1721, October 1963. 11

[45] A. Ghosh and D. Dhar. On the orientational ordering of long rods on a lattice. EPL (Europhysics Letters), pages 20003+, April 2007. 11

[46] D. H. Linares, F. Romá, and A. J. Ramirez-Pastor. Entropy-driven phase transition in a system of long rods on a square lattice. Journal of Statistical Mechanics: Theory and Experiment, 2008(03):P03013+, March 2008. 11

[47] D. A. Matoz-Fernandez, D. H. Linares, and A. J. Ramirez-Pastor. Critical behavior of long straight rigid rods on two-dimensional lattices: Theory and Monte Carlo simulations. The Journal of Chemical Physics, 128(21):214902, 2008. 11

[48] D. A. Matoz-Fernandez, D. H. Linares, and A. J. Ramirez-Pastor. Determination of the critical exponents for the isotropic-nematic phase transition in a system of long rods on two-dimensional lattices: Universality of the transition. EPL (Europhysics Letters), pages 50007+, June 2008. 11

[49] T. Fischer and R. L. C. Vink. Restricted orientation. EPL (Europhysics Letters), pages 56003+, March 2009. 11, 17, 44

[50] T. Sjöstrand, S. Mrenna, and P. Skands. PYTHIA 6.4 physics and manual. Journal of High Energy Physics, 5:26, May 2006. 11

[51] T. Gleisberg, S. Hoeche, F. Krauss, A. Schaelicke, S. Schumann, and J. Winter. SHERPA 1.alpha, a proof-of-concept version. Journal of High Energy Physics, 2:56, February 2004. 11

[52] W. Morokoff. Quasi-Monte Carlo Integration. Journal of Computational Physics, 122:218-230, December 1995. 12

[53] M. E. J. Newman and G. T. Barkema. Monte Carlo Methods in Statistical Physics. Oxford University Press, USA, April 1999. 12, 13, 27, 44, 76

[54] W. K. Hastings. Monte Carlo sampling methods using Markov chains and their applications. Biometrika, 57(1):97-109, 1970. 13

[55] N. Metropolis, A. Rosenbluth, A. Teller, and E. Teller. Equation of State Calculations by Fast Computing Machines. Journal of Chemical Physics, 21(6):1087-1092, 1953. 16

[56] R. L. C. Vink and J. Horbach. Grand canonical Monte Carlo simulation of a model colloid-polymer mixture: Coexistence line, critical behavior, and interfacial tension. The Journal of Chemical Physics, 121(7):3253-3258, 2004. 17 
[57] F. Oosawa and S. Asakura. Surface Tension of High-Polymer Solutions. Journal of Chemical Physics, 22:1255, July 1954. 17

[58] J. Machta, Y. S. Choi, A. Lucke, T. Schweizer, and L. V. Chayes. Invaded Cluster Algorithm for Equilibrium Critical Points. Phys. Rev. Lett., 75(15):2792-2795, October 1995. 17

[59] Lars Onsager. Crystal Statistics. I. A Two-Dimensional Model with an OrderDisorder Transition. Phys. Rev., 65:117-149, February 1944. 22

[60] R. Guida and J. Zinn-Justin. Critical exponents of the N-vector model. Journal of Physics A: Mathematical and General, 31(40):8103, 1998. 22

[61] J. Zinn-Justin. Precise determination of critical exponents and equation of state by field theory methods. Physics Reports, 344(4-6):159-178, April 2001. 22, 28

[62] K. Binder and D. P. Landau. Finite-size scaling at first-order phase transitions. Phys. Rev. B, 30:1477-1485, August 1984. 25

[63] Alan M. Ferrenberg and Robert H. Swendsen. New Monte Carlo technique for studying phase transitions. Physical Review Letters, 61(23):2635+, December 1988. 33

[64] Bernd A. Berg and Thomas Neuhaus. Multicanonical ensemble: A new approach to simulate first-order phase transitions. Physical Review Letters, 68(1):9+, January 1992. 36,54

[65] Fugao Wang and D. P. Landau. Efficient, Multiple-Range Random Walk Algorithm to Calculate the Density of States. Physical Review Letters, 86(10):2050+, March 2001. 36,37

[66] Qiliang Yan and Juan J. de Pablo. Fast Calculation of the Density of States of a Fluid by Monte Carlo Simulations. Physical Review Letters, 90(3):035701+, January 2003. 37

[67] M. S. Shell, P. G. Debenedetti, and A. Z. Panagiotopoulos. An improved Monte Carlo method for direct calculation of the density of states. Journal of Chemical Physics, 119:9406-9411, November 2003. 37

[68] Ruben G. Ghulghazaryan, Shura Hayryan, and Chin-Kun Hu. Efficient combination of WangLandau and transition matrix Monte Carlo methods for protein simulations. Journal of Computational Chemistry, 28(3):715-726, 2007. 37

[69] G. M. Torrie and J. P. Valleau. Nonphysical sampling distributions in Monte Carlo free-energy estimation: Umbrella sampling. Journal of Computational Physics, 23(2):187-199, 1977. 37 
[70] Peter Virnau and Marcus Müller. Calculation of free energy through successive umbrella sampling. The Journal of Chemical Physics, 120(23):10925-10930, 2004. 38

[71] B. J. Schulz, K. Binder, M. Müller, and D. P. Landau. Avoiding boundary effects in Wang-Landau sampling. Phys. Rev. E, 67:067102, June 2003. 38

[72] T. Chonavel. Statistical Signal Processing: Modelling and Estimation. Springer, 1 edition, April 2002. 43

[73] Alan M. Ferrenberg and Robert H. Swendsen. New Monte Carlo Technique for Studying Phase Transitions. Phys. Rev. Lett., 63:1658, October 1989. 44

[74] Shankar Kumar, John M. Rosenberg, Djamal Bouzida, Robert H. Swendsen, and Peter A. Kollman. The weighted histogram analysis method for free-energy calculations on biomolecules. I. The method. Journal of Computational Chemistry, 13(8):1011-1021, 1992. 44

[75] L. G. MacDowell, V. K. Shen, and J. R. Errington. Nucleation and cavitation of spherical, cylindrical, and slablike droplets and bubbles in small systems. Journal of Chemical Physics, 125(3), July 2006. 49

[76] Samuel Safran. Statistical Thermodynamics Of Surfaces, Interfaces, And Membranes (Frontiers in Physics). Westview Press, January 2003. 51

[77] K. Binder. Monte Carlo calculation of the surface tension for two- and threedimensional lattice-gas models. Physical Review A, 25(3):1699+, March 1982. 51

[78] Thomas Neuhaus and Johannes S. Hager. 2D Crystal Shapes, Droplet Condensation, and Exponential Slowing Down in Simulations of First-Order Phase Transitions. Journal of Statistical Physics, 113:47-83, 2003. 52, 54, 57

[79] K. Leung and R. K. P. Zia. Geometrically induced transitions between equilibrium crystal shapes. Journal of Physics A: Mathematical and General, 23(20):4593-4602, 1990. 53

[80] Bernd A. Berg and Thomas Neuhaus. Multicanonical algorithms for first order phase transitions. Physics Letters B, 267(2):249-253, 1991. 54

[81] L. A. Bagatolli and E. Gratton. Two Photon Fluorescence Microscopy of Coexisting Lipid Domains in Giant Unilamellar Vesicles of Binary Phospholipid Mixtures. Biophysical Journal, 78(1):290-305, 2000. 62, 100, 105

[82] L. A. Bagatolli and E. Gratton. Two-Photon Fluorescence Microscopy Observation of Shape Changes at the Phase Transition in Phospholipid Giant Unilamellar Vesicles. Biophysical Journal, 77(4):2090-2101, 1999. 62 
[83] Nicoletta Kahya, Dag Scherfeld, Kirsten Bacia, and Petra Schwille. Lipid domain formation and dynamics in giant unilamellar vesicles explored by fluorescence correlation spectroscopy. Journal of Structural Biology, 147(1):77-89, 2004. 62

[84] Sarah L. Veatch and Sarah L. Keller. Separation of Liquid Phases in Giant Vesicles of Ternary Mixtures of Phospholipids and Cholesterol. Biophysical Journal, 85(5):3074-3083, 2003. 62, 100, 105

[85] H. H. Wensink and R. L. C. Vink. First-order phase transitions in two-dimensional off-lattice liquid crystals. Journal of Physics: Condensed Matter, 19(46):466109+, November 2007. 62

[86] J. M. Caillol and D. Levesque. Numerical simulations of homogeneous and inhomogeneous ionic systems: An efficient alternative to the Ewald method. Journal of Chemical Physics, 94:597-607, January 1991. 63

[87] Reimer Kühn. Critical Behavior of the Randomly Spin Diluted 2D Ising Model: A Grand Ensemble Approach. Phys. Rev. Lett., 73(16):2268-2271, October 1994. 65, 73

[88] Giorgio Mazzeo and Reimer Kühn. Critical behavior of the two-dimensional spindiluted Ising model via the equilibrium ensemble approach. Phys. Rev. E, 60:38233836, October 1999. 65, 73

[89] Jae-Kwon Kim and Adrian Patrascioiu. Critical behavior of the specific heat in the two dimensional site diluted Ising system. Phys. Rev. Lett., 72:2785-2788, April 1994. 65

[90] H. G. Ballesteros, L. A. Fernández, V. Martín-Mayor, A. Muñoz Sudupe, G. Parisi, and J. J. Ruiz-Lorenzo. Ising exponents in the two-dimensional site-diluted Ising model. Journal of Physics A: Mathematical and General, 30(24):8379+, December 1997. 65

[91] P. H. L. Martins and J. A. Plascak. Universality class of the two-dimensional site-diluted Ising model. Phys. Rev. E, 76:012102, July 2007. 65

[92] R. Kenna and J. J. Ruiz-Lorenzo. Scaling analysis of the site-diluted Ising model in two dimensions. Phys. Rev. E, 78:031134, September 2008. 65

[93] M. Fähnle, T. Holey, and J. Eckert. Monte Carlo renormalization group calculations of critical exponents in site-diluted 2D and 3D Ising systems. Journal of Magnetism and Magnetic Materials, 104-107, Part 1(0):195-196, 1992. 65

[94] C. Monthus and T. Garel. Distribution of pseudo-critical temperatures and lack of self-averaging in disordered Poland-Scheraga models with different loop exponents. Eur. Phys. J. B, 48(3):393-403, December 2005. 71 
[95] J. M. Yeomans and R. B. Stinchcombe. Critical properties of site- and bond-diluted Ising ferromagnets. Journal of Physics C: Solid State Physics, 12(2):347+, January 1979. 73

[96] Pier G. De Sanctis Lucentini and Giuseppe Pellicane. Critical Behavior of Symmetrical Fluid Mixtures in Random Pores. Phys. Rev. Lett., 101:246101, December 2008. 74

[97] John Z. Imbrie. Lower Critical Dimension of the Random-Field Ising Model. Phys. Rev. Lett., 53(18):1747-1750, October 1984. 75, 87

[98] J. Bricmont and A. Kupiainen. Lower critical dimension for the random-field Ising model. Phys. Rev. Lett., 59(16):1829-1832, October 1987. 75, 87

[99] J. Machta, M. E. J. Newman, and L. B. Chayes. Replica-exchange algorithm and results for the three-dimensional random field Ising model. Phys. Rev. E, 62:8782-8789, December 2000. 78

[100] T. Fischer and R. L. C. Vink. Fluids with quenched disorder: scaling of the free energy barrier near critical points. Journal of Physics: Condensed Matter, 23(23):234117+, June 2011. 79, 82, 84, 85, 111

[101] R. L. C. Vink, T. Neuhaus, and H. Löwen. Fluid phase separation inside a static periodic field: An effectively two-dimensional critical phenomenon. Journal of Chemical Physics, 134(20), May 2011. 79

[102] R. L. C. Vink and A. J. Archer. Phase separation in fluids exposed to spatially periodic external fields. ArXiv e-prints, 1109.6746, September 2011. 79

[103] Daniel S. Fisher. Scaling and critical slowing down in random-field Ising systems. Phys. Rev. Lett., 56:416-419, February 1986. 81, 82

[104] B. Widom. Surface Tension and Molecular Correlations near the Critical Point. Journal of Chemical Physics, 43:3892-3897, December 1965. 82

[105] Yoseph Imry and Shang-keng Ma. Random-Field Instability of the Ordered State of Continuous Symmetry. Phys. Rev. Lett., 35:1399-1401, November 1975. 87

[106] Dimiter S. Dimitrov. Liposome electroformation. Faraday Discuss. Chem. Soc., 81:303-311, 1986. 100

[107] Fredric M. Menger and Jason S. Keiper. Chemistry and physics of giant vesicles as biomembrane models. Current Opinion in Chemical Biology, 2(6):726-732, 1998. 100

[108] R. E. Scott. Plasma Membrane Vesiculation: A New Technique for Isolation of Plasma Membranes. Science, 194:743-745, November 1976. 100 
[109] David Holowka and Barbara Baird. Structural studies on the membrane-bound immunoglobulin E-receptor complex. 1. Characterization of large plasma membrane vesicles from rat basophilic leukemia cells and insertion of amphipathic fluorescent probes. Biochemistry, 22(14):3466-3474, 1983. 100

[110] T. Baumgart, A. T. Hammond, P. Sengupta, S. T. Hess, D. A. Holowka, B. A. Baird, and W. W. Webb. Large-scale fluid/fluid phase separation of proteins and lipids in giant plasma membrane vesicles. Proceedings of the National Academy of Science, 104:3165-3170, February 2007. 100

[111] Benjamin B. Machta, Stefanos Papanikolaou, James P. Sethna, and Sarah L. Veatch. Minimal Model of Plasma Membrane Heterogeneity Requires Coupling Cortical Actin to Criticality. Biophysical Journal, 100(7):1668-1677, 2011. 100, 102

[112] Matthew S. Turner, Pierre Sens, and Nicholas D. Socci. Nonequilibrium Raftlike Membrane Domains under Continuous Recycling. Phys. Rev. Lett., 95:168301, October 2005. 102

[113] Jens Ehrig, Eugene P. Petrov, and Petra Schwille. Near-Critical Fluctuations and Cytoskeleton-Assisted Phase Separation Lead to Subdiffusion in Cell Membranes. Biophysical Journal, 100(1):80-89, 2011. 102

[114] Udo Seifert. Configurations of fluid membranes and vesicles. Advances in Physics, 46(1):13-137, 1997. 102

[115] David Van Der Spoel, Erik Lindahl, Berk Hess, Gerrit Groenhof, Alan E. Mark, and Herman J. C. Berendsen. GROMACS: Fast, flexible, and free. Journal of Computational Chemistry, 26(16):1701-1718, 2005. 103

[116] Berk Hess, Carsten Kutzner, David van der Spoel, and Erik Lindahl. GROMACS 4: Algorithms for Highly Efficient, Load-Balanced, and Scalable Molecular Simulation. Journal of Chemical Theory and Computation, 4(3):435-447, 2008. 103

[117] H. Jelger Risselada and Siewert J. Marrink. The molecular face of lipid rafts in model membranes. Proceedings of the National Academy of Sciences, 105(45):17367-17372, November 2008. 103, 106

[118] Siewert J. Marrink, H. Jelger Risselada, Serge Yefimov, D. Peter Tieleman, and Alex H. de Vries. The MARTINI Force Field: Coarse Grained Model for Biomolecular Simulations. The Journal of Physical Chemistry B, 111(27):7812-7824, 2007. 103, 105 